\title{
Zusammenhang von Persönlichkeit, Führungsverhalten und Erfolg von Fitnesscenter-Managern
}

\author{
Dissertation \\ Zur Erlangung des sozialwissenschaftlichen Doktorgrades des \\ Fachbereichs Sozialwissenschaften der Universität Göttingen
}

Vorgelegt von

Dieter Schmid

aus Schlattingen (TG), Schweiz

Göttingen, 2000 
Berichterstatter:

Mitberichterstatter:

Tag der mündlichen Prüfung:
Prof. Dr. Arnd Krüger

Prof. Dr. Axel Dreyer

24. Februar 2000 


\section{Widmung}

Für meine Eltern Beatrice und Heinz, die mich immer gefördert und unterstützt haben.

\section{Vorbemerkung}

Es wird zur Vereinfachung für die Leserin und den Leser jeweils die männliche Form verwendet, auch wenn damit gleichzeitig die weibliche Person gemeint ist.

Umgekehrt ist bei der Verwendung der weiblichen Form auch die männliche Person eingeschlossen.

\section{Erklärung}

Ich versichere, dass ich die eingereichte Dissertation ,Zusammenhang von Persönlichkeit, Führungsverhalten und Erfolg von Fitnesscenter-Managern“" selbständig und ohne unerlaubte Hilfsmittel verfasst habe. Anderer, als der von mir angegebenen Hilfsmittel und Schriften habe ich mich nicht bedinet. Alle wörtlichen oder sinngemäss den Schriften anderer Autoren entnommenen Stellen habe ich kenntlich gemacht.

Schaffhausen, im März 2000 


\section{Inhaltsverzeichnis}

1 Einleitung --:-and

\begin{tabular}{|lll}
\hline $1.1 \quad$ Problemstellung & 1 \\
\hline
\end{tabular}

\begin{tabular}{|lll}
\hline $1.2 \quad$ Wachstumsfaktoren der Fitnessbranche & 3 \\
\hline
\end{tabular}

1.2.1 $\quad$ Entwicklung des Fitnessbewusstseins in der Bevölkerung 33

1.2.2 Persönliche Fitness als Mittel der Sinn- und Identitätsfindung 33

1.2.3 Die Stilisierung des perfekten Körpers 5

\begin{tabular}{|lll}
\hline 1.3 Vorgehen & 6 \\
\hline
\end{tabular}

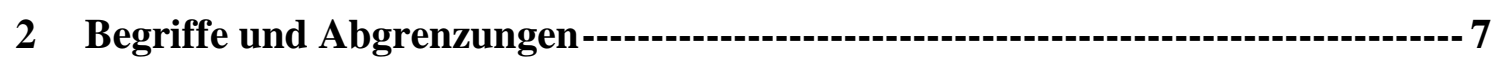

\begin{tabular}{|lll}
\hline $2.1 \quad$ Begriffe & 7 \\
\hline 2.2 & Abgrenzungen
\end{tabular}

2.2 Abgrenzungen 12

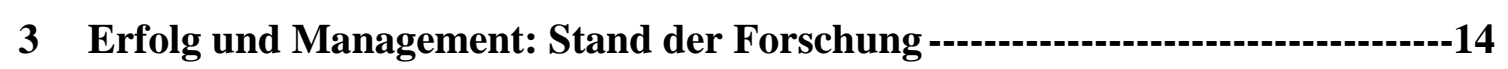

\begin{tabular}{lll}
$3.1 \quad$ Persönlichkeit und Erfolg & 14 \\
\hline
\end{tabular}

\begin{tabular}{lll}
3.2 & Situation und Erfolg & 14 \\
\hline 3.3 & Verhalen und Erfolg & 15
\end{tabular}

\begin{tabular}{lll}
3.3 & Verhalten und Erfolg & 15 \\
\hline
\end{tabular}

$3.4 \quad$ Person und Situation und Erfolg 15

\begin{tabular}{lll}
\hline .5 & Trainerpersönlichkeit und Erfolg & 18 \\
\hline
\end{tabular}

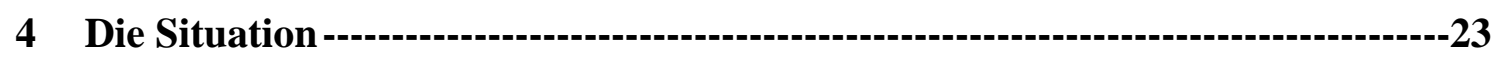

\begin{tabular}{|lll}
\hline $4.1 \quad$ Die Fitnesslandschaft Schweiz & 23 \\
\hline
\end{tabular}

\begin{tabular}{|ll}
\hline .2 & Der typische Fitnesscenterbesucher
\end{tabular}

\begin{tabular}{lll}
\hline 4.3 Qualitop & 26 \\
\hline
\end{tabular}

\begin{tabular}{|lll}
\hline 4.4 & Situative Variablen der vorliegenden Untersuchung & 27 \\
\hline
\end{tabular}

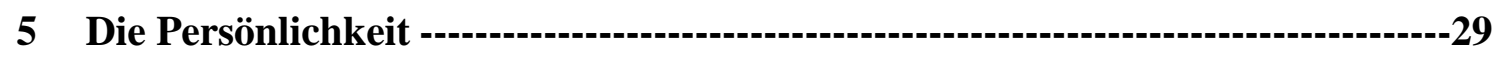

\begin{tabular}{|c|c|c|}
\hline $5.1 \quad E$ & urs zur Persönlichkeitspsychologie & 29 \\
\hline 5.1 .1 & Personale Ansätze & 29 \\
\hline 5.1 .2 & Situative Ansätze & 29 \\
\hline 5.1 .3 & Interaktionistische Ansätze & 29 \\
\hline
\end{tabular}




\begin{tabular}{|llr|}
\hline 5.3 & Typologien & 30 \\
\hline 5.3 .1 & Strong Interest Inventory & 30 \\
\hline 5.3 .2 & Myers-Briggs Type Indicator MBTI & 31 \\
\hline 5.3 .3 & Weitere Management-spezifische Typologien & 31 \\
\hline 5.4 & Auswahl des Erhebungsinstruments & 31 \\
\hline 5.5 & Die verschiedenen Typen des MBTI & 33 \\
\hline
\end{tabular}

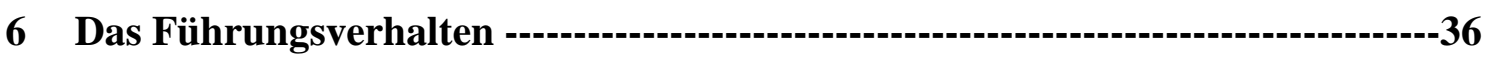

\begin{tabular}{lll}
\hline $6.1 \quad$ Der analytisch-funktionsorientierte Ansatz & 36 \\
\hline
\end{tabular}

6.2 Der empirisch-handlungsorientierte Ansatz 36

\begin{tabular}{lll}
\hline $6.3 \quad$ Auswahl des Erhebungsinstruments & 39 \\
\hline
\end{tabular}

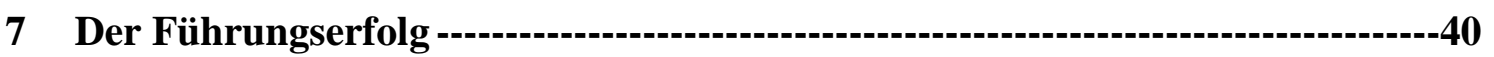

\begin{tabular}{lll}
\hline $.1 \quad$ Problematik der Erfolgsmessung & 40 \\
\hline
\end{tabular}

\begin{tabular}{|ll|}
\hline $7.2 \quad$ Aufbau eines Messsystems & 40 \\
\hline
\end{tabular}

\begin{tabular}{|lr}
\hline $.3 \quad$ Auswahl der Erhebungskriterien & 41 \\
\hline
\end{tabular}

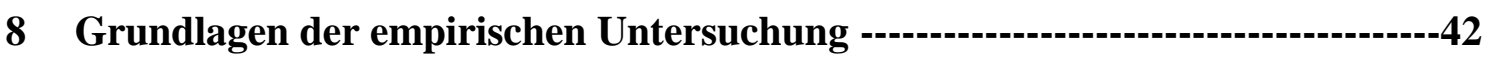

$8.1 \quad$ Baustein Persönlichkeit $\quad 42$

\begin{tabular}{llr}
$8.2 \quad$ Baustein Führungsverhalten & 42 \\
\hline
\end{tabular}

\begin{tabular}{llr} 
8.2.1 & Konkretisierungskriterien der 10 Führungsrollen im Fitnesscenter & 42 \\
\hline
\end{tabular}

\begin{tabular}{lll}
\hline 8.2 .2 & Vernehmlassung durch Branchenexperten & 44 \\
\hline
\end{tabular}

\begin{tabular}{lll}
8.2 .3 & Liste der bereinigten Rollenkriterien & 45 \\
\hline 8.2 .4 & 46
\end{tabular}

\begin{tabular}{lll}
8.2 .4 & Inhalt des Fragebogenteils „,Führungstätigkeiten“ & 46 \\
\hline
\end{tabular}

\begin{tabular}{lll}
\hline 8.2 .5 & Überprüfung des Gesamtsystems & 47 \\
\hline
\end{tabular}

\begin{tabular}{llr}
$8.3 \quad$ Baustein Erfolg & 47 \\
\hline
\end{tabular}

\begin{tabular}{lll}
8.3 .1 & Identifikation des Umfelds und möglicher Messgrössen & 47 \\
\hline
\end{tabular}

\begin{tabular}{lll}
\hline 8.3.2 & Analyse der Eigenschaften der möglichen Massgrössen & 48 \\
\hline
\end{tabular}

\begin{tabular}{lll}
8.3 .3 & Entwicklung und Analyse neuer Massgrössen & 49 \\
\hline .3 .4 & Ubepring
\end{tabular}

\begin{tabular}{lll}
8.3 .4 & Überprüfung des Gesamtsystems & 49 \\
\hline
\end{tabular}

\begin{tabular}{llr}
$8.4 \quad$ Baustein Situation & 49 \\
\hline
\end{tabular}

\begin{tabular}{llr}
8.4 .1 & Identifikation möglicher Massgrössen & 49 \\
\hline
\end{tabular}

\begin{tabular}{llr}
\hline 8.4 .2 & Analyse der Variablen „Konkurrenz-Situation“ & 50 \\
\hline
\end{tabular}

8.4.3 „Mitarbeiter-Situation“ und „Wirtschaftliche und Gesellschaftliche Situation“ 51

8.4.4 $\quad$ Analyse der übrigen Variablen 51

\begin{tabular}{lll}
8.4 .5 & Entwicklung und Analyse neuer Massgrössen & 54 \\
\hline 8.4 .6 & Ubepron & 54 \\
\hline
\end{tabular}

\begin{tabular}{lll}
\hline 8.4 .6 & Überprüfung des Gesamtsystems & 54 \\
\hline
\end{tabular} 


\begin{tabular}{|c|c|c|}
\hline 8.5 & ersicht über die Untersuchungsanlage & 55 \\
\hline 8.5 .1 & Gesamtübersicht & 55 \\
\hline 8.5 .2 & Übersicht über die Datenquellen & 55 \\
\hline 8.6 & swertungsmöglichkeiten im Überblick & 56 \\
\hline 8.7 & swertungen der einzelnen Teilbereiche (Nummern 1 - 4) & 57 \\
\hline 8.7 .1 & Persönlichkeit: MBTI (1) & 57 \\
\hline 8.7 .2 & Führungsverhalten (2) & 57 \\
\hline 8.7 .3 & Erfolg (3) & 57 \\
\hline 8.7 .4 & Situation (4) & 57 \\
\hline $8.8 \quad A$ & swertung der Zusammenhänge (Nummern 5 - 8) & 57 \\
\hline 8.8 .1 & Auswirkung der Persönlichkeit auf das Führungsverhalten (5) & 57 \\
\hline 8.8 .2 & Auswirkung des Führungsverhaltens auf den Erfolg (6) & 57 \\
\hline 8.8 .3 & Auswirkung der Persönlichkeit auf den Erfolg (7) & 58 \\
\hline 8.8 .4 & Auswirkung der Situation auf den Erfolg (8) & 58 \\
\hline
\end{tabular}

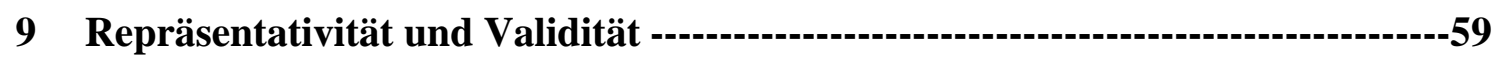

\begin{tabular}{llr}
$9.1 \quad$ Geographische Verteilung & 59 \\
\hline
\end{tabular}

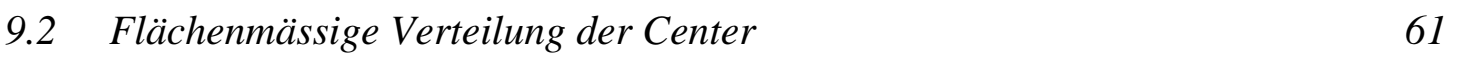

\begin{tabular}{lll}
9.3 & Geschlechtsverteilung der Centerleitenden & 62 \\
\hline 0.40
\end{tabular}

$9.4 \quad$ Validitätsprüfung anhand der Konkurrenzsituation 63

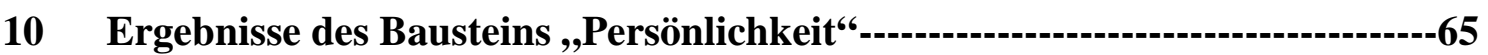

\begin{tabular}{llr|}
\hline 10.1 & Verteilung der 16 Persönlichkeitstypen & 65 \\
\hline 10.2 & Verteilung auf die vier Ausprägungen $E-I, N-S, T-F$ und J-P & 65 \\
\hline 10.2 .1 & Der Prototyp eines Fitnesscenter-Managers & 67 \\
\hline 10.2 .2 & Der Gegentyp zum Prototyp & 71 \\
\hline 10.3 & Verteilung der vier Kerntypen $S T, S F, N T$ und $N F$ & 72 \\
\hline $10.4 \quad$ Zusammenfassung ,Persönlichkeit“ & 74 \\
\hline
\end{tabular}

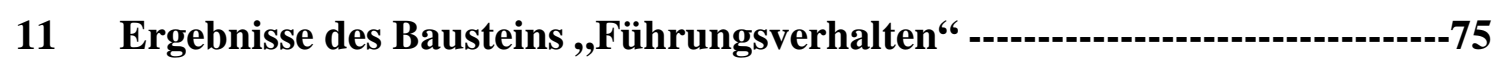

$11.1 \quad$ Wichtigkeit der Führungstätigkeiten für den Erfolg 75

\begin{tabular}{lll}
\hline $1.2 \quad$ Zeitaufwand für die einzelnen Führungstätigkeiten & 79
\end{tabular}

$11.3 \quad$ Führungsverhalten: Wichtigkeit für den Erfolg und Zeitaufwand 80

\begin{tabular}{|ll}
\hline $1.4 \quad$ Zusammenfassung „Führungsverhalten“ & 81 \\
\hline
\end{tabular} 


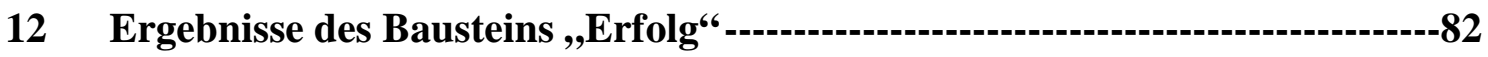

\begin{tabular}{|llr|}
\hline 12.1 & Persönliche Erfolgsbeurteilung & 82 \\
\hline 12.2 & Centerfläche & 83 \\
\hline 12.3 & Mitgliederzahlen & 84 \\
\hline 12.4 & Einzeleintritte & 85 \\
\hline 12.5 & Entwicklung der Rendite & 85 \\
\hline 12.6 & Investitionen während der letzten drei Jahre & 86 \\
\hline 12.7 & Detaillierte Erfolgsbeurteilung & 87 \\
\hline 12.8 & Persönliche Erklärungen für den Erfolg & 90 \\
\hline 12.9 & Erfolgsfaktor Effizienz & 95 \\
\hline 12.10 & Zusammenfassung „Erfolg“ & 98 \\
\hline
\end{tabular}

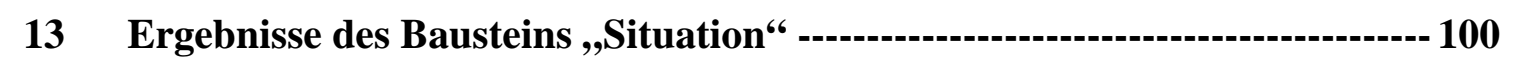

\begin{tabular}{|llr}
\hline $13.1 \quad$ Konkurrenzdruck & 100 \\
\hline
\end{tabular}

\begin{tabular}{|lll}
\hline $13.2 \quad$ Erfolgsfaktoren aus Kundensicht & 101 \\
\hline
\end{tabular}

\begin{tabular}{|lll}
\hline $13.3 \quad$ Erfüllung der Kundenanforderungen & 104
\end{tabular}

\begin{tabular}{|lll}
\hline 13.4 & Zusammenfassung ,Situation“ & 107
\end{tabular}

14 Zusammenhänge zwischen Persönlichkeit und Führungsverhalten --------- 108

\begin{tabular}{|llll}
\hline 14.1 & Genereller Zusammenhang von Persönlichkeit und Führungsverhalten 108 \\
\hline
\end{tabular}

\begin{tabular}{|lll}
\hline $14.2 \quad$ Führungsverhalten der Ausprägung E-I & 109 \\
\hline
\end{tabular}

\begin{tabular}{|lll|}
\hline $14.3 \quad$ Führungsverhalten der Ausprägung $S-N$ & 111 \\
\hline
\end{tabular}

\begin{tabular}{|lll|}
\hline $14.4 \quad$ Führungsverhalten der Ausprägung T-F & 113 \\
\hline
\end{tabular}

\begin{tabular}{|lll}
\hline 14.5 & Führungsverhalten der Ausprägung J-P & 114 \\
\hline
\end{tabular}

\begin{tabular}{|lll}
\hline $14.6 \quad$ Zeitaufwand-Extremwerte für die einzelnen Führungstätigkeiten & 115 \\
\hline
\end{tabular}

14.7 Zusammenfassung „Persönlichkeit $\leftrightarrow \rightarrow$ Führungsverhalten“ 117

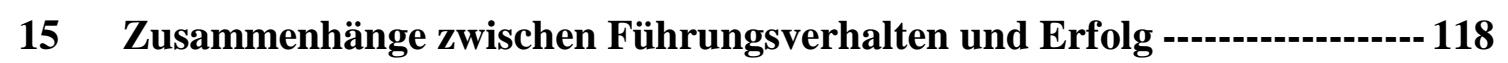

\begin{tabular}{|lll}
\hline 15.1 & Führungsverhalten und Erfolg als Ganzes & 118 \\
\hline
\end{tabular}

\begin{tabular}{|lll}
\hline $15.2 \quad$ Führungsverhalten und Erfolg zweifach kategorisiert & 118 \\
\hline
\end{tabular}

\begin{tabular}{|lll|}
\hline $15.3 \quad$ Extremwerte Führungsverhalten und Erfolg (vierfach kategorisiert) & 119 \\
\hline
\end{tabular}

115.4 Führungsverhalten des sehr erfolgreichen Managers 120

$15.5 \quad$ Zusammenfassung ,Führungsverhalten $\leftarrow \rightarrow$ Erfolg 121 


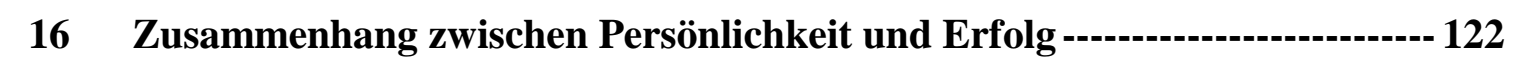

\begin{tabular}{|lll}
\hline 16.1 & Rangliste der 16 Einzeltypen & 122 \\
\hline
\end{tabular}

\begin{tabular}{|lll}
\hline $16.2 \quad$ Rangliste der Kerntypen & 123 \\
\hline
\end{tabular}

\begin{tabular}{|lll}
\hline 16.3 & Rangliste der 4 Ausprägungen nach Erfolg & 124 \\
\hline
\end{tabular}

\begin{tabular}{|lll}
\hline $16.4 \quad$ Der Erfolgstyp ESTJ & 124 \\
\hline
\end{tabular}

\begin{tabular}{|lll}
\hline 16.5 & Persönlichkeit und Erfolgskennzahlen & 128 \\
\hline
\end{tabular}

16.6 Zusammenfassung „,Persönlichkeit $\leftarrow \rightarrow$ Erfolg“ 128

17 Zusammenhänge zwischen Situation und Erfolg -----------------------------129

\begin{tabular}{|lll}
\hline $17.1 \quad$ Rangliste der situativen Erfolgsfaktoren & 129 \\
\hline
\end{tabular}

$17.2 \quad$ Erfüllung der Erfolgsfaktoren durch die einzelnen Center 130

17.3 Zusammenfassung „Situation $\leftarrow \rightarrow$ Erfolg“ 131

18 Zusammenhänge zwischen Persönlichkeit und Situation -------------------- 132

\begin{tabular}{|lll|}
\hline $18.1 \quad$ Erfüllung der situativen Erfolgsfaktoren durch die einzelnen Typen & 132 \\
\hline
\end{tabular}

18.2 Zusammenfassung „Persönlichkeit $\leftrightarrow \rightarrow$ Situation“ 133

19 Unterschiede Stadt-Land und Gross-Klein --------------------------------- 134

\begin{tabular}{|c|c|c|}
\hline 19.1 & Unterschiede Stadt - Land & 134 \\
\hline 19.1 .1 & Verteilung & 134 \\
\hline 19.1 .2 & Grösse & 135 \\
\hline 19.1 .3 & Anzahl Kunden & 136 \\
\hline 19.1 .4 & Manager-Persönlichkeit & 137 \\
\hline 19.1 .5 & Konkurrenzdruck & 137 \\
\hline 19.1 .6 & Personal & 138 \\
\hline 19.1 .7 & Führungsverhalten & 139 \\
\hline 19.1 .8 & Erfolg & 140 \\
\hline 19.1 .9 & Zusammenfassung & 140 \\
\hline 19.2 & Unterschiede Gross - Klein & 141 \\
\hline 19.2 .1 & Verteilung & 141 \\
\hline 19.2 .2 & Manager-Persönlichkeit & 141 \\
\hline 19.2 .3 & Konkurrenzdruck & 142 \\
\hline 19.2 .4 & $\begin{array}{c}\text { Personal } \\
\end{array}$ & 143 \\
\hline 19.2 .5 & Führungsverhalten & 143 \\
\hline 19.2 .6 & Erfolg & 145 \\
\hline 19.2 .7 & Zusammenfassung & 145 \\
\hline
\end{tabular}




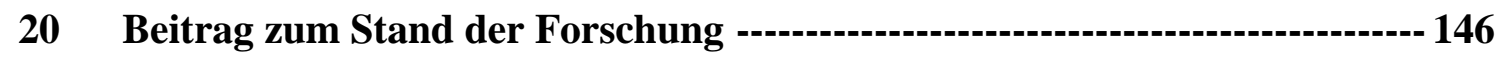

20.1 Erkenntnisse über die Wachstumsfaktoren der Branche 146

\begin{tabular}{|lr|}
\hline $20.2 \quad$ Erkenntnisse über die Übertragbarkeit der Ergebnisse & 149 \\
\hline
\end{tabular}

\begin{tabular}{lll}
20.2 .1 & Management-Anforderungen in der Dienstleistungsbranche & 149 \\
\hline
\end{tabular}

20.2.2 Gesellschaftliche Veränderungen und ihre Auswirkungen 151

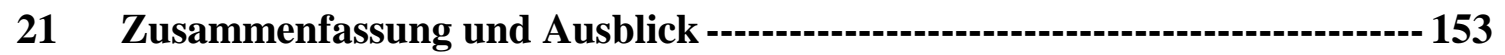

21.1 Erkenntnisse über die Zusammenhänge zwischen den einzelnen Bausteinen153

$21.2 \quad$ Vergleich der Gruppen Stadt - Land und Gross - Klein 156

21.3 Erkenntnisse über die Wachstumsfaktoren der Branche 158

$21.4 \quad$ Erkenntnisse über die Übertragbarkeit der Ergebnisse 159

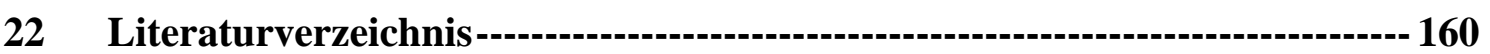

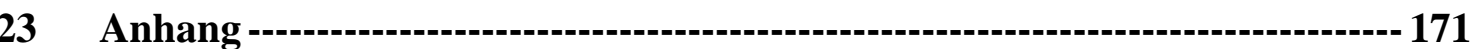




\section{Abbildungsverzeichnis}

\begin{tabular}{|ll}
\hline Abbildung 1-1 Kategorien der Selbstkontrolle von Fitnesscenter-Besuchern & 5 \\
\hline
\end{tabular}

Abbildung 2-1 Fitnesskonzepte (nach KAYSER, 1997) 8

\begin{tabular}{|ll|}
\hline Abbildung 3-1 Bedingtheit des Erfolges durch Person und Situation & 16 \\
\hline Abbildung 3-2 Art & 17 \\
\hline
\end{tabular}

\begin{tabular}{|lr|}
\hline Abbildung 3-2 Arbeitsmodell der vorliegenden Arbeit & 17 \\
\hline Abbildung 3-3 Multidimensionales Fuhrungsmodel fir den Sport & 18 \\
\hline
\end{tabular}

Abbildung 3-3 Multidimensionales Führungsmodell für den Sport 18

\begin{tabular}{|lr|}
\hline Abbildung 5-1 Die beiden Typen der Ausprägung E - I & 33 \\
\hline Abbildung 5-2 Die beiden Typen & 33 \\
\hline
\end{tabular}

\begin{tabular}{|lr|}
\hline Abbildung 5-2 Die beiden Typen der Ausprägung N - S & 33 \\
\hline Abbildung 5-3 Die beiden Typen
\end{tabular}

\begin{tabular}{|lr|}
\hline Abbildung 5-3 Die beiden Typen der Ausprägung T - F & 33 \\
\hline Abbildung 5-4 Die beiden Typen & 34 \\
\hline
\end{tabular}

\begin{tabular}{|lr|}
\hline Abbildung 5-4 Die beiden Typen der Ausprägung J - P & 34 \\
\hline Abbildung 5-5 Die vier Kerntypen
\end{tabular}

\begin{tabular}{|lr|}
\hline Abbildung 5-5 Die vier Kerntypen & 34 \\
\hline
\end{tabular}

\begin{tabular}{|lr|}
\hline Abbildung 6-1 Die 10 Führungsrollen & 37 \\
\hline Abbildung 6-2 Die 12 & 38 \\
\hline
\end{tabular}

\begin{tabular}{|rr|}
\hline Abbildung 6-2 Die 12 LOS-Kategorien & 38 \\
\hline
\end{tabular}

Abbildung 7-1 Kriterien der 10 Führungsrollen eines Fitnesscenter-Managers 44

\begin{tabular}{|lr|}
\hline Abbildung 8-1 Geographische Verteilung der Center & 60 \\
\hline Abbildung 8-2 Geographische Verteilung der Center in $\%$ & 60 \\
\hline
\end{tabular}

\begin{tabular}{|lr|}
\hline Abbildung 8-2 Geographische Verteilung der Center in \% & 60 \\
\hline Abbildung 8-3 & 61 \\
\hline
\end{tabular}

\begin{tabular}{|lr|}
\hline Abbildung 8-3 Flächenmässige Verteilung der Center & 61 \\
\hline
\end{tabular}

\begin{tabular}{ll}
\hline Abbildung 8-4 Geschlechterverteilung der Center-Leitenden & 63 \\
\hline
\end{tabular}

\begin{tabular}{|lc|}
\hline Abbildung 9-1 Verteilung der Persönlichkeitstypen & 65 \\
\hline Abbildung 9-2 Verteilung der Ausprägungen & 66 \\
\hline
\end{tabular}

\begin{tabular}{|cc|}
\hline Abbildung 9-2 Verteilung der Ausprägungen & 66 \\
\hline Abbildung 9-3 Verteilung & 69 \\
\hline
\end{tabular}

\begin{tabular}{ll}
\hline Abbildung 9-3 Verteilung der Persönlichkeitstypen im Vergleich I & 69 \\
\hline Abbidung 9-4 Verteilung
\end{tabular}

\begin{tabular}{ll}
\hline Abbildung 9-4 Verteilung der Persönlichkeitstypen im Vergleich II & 70 \\
\hline Abbidung 9-5 Ver.
\end{tabular}

\begin{tabular}{ll}
\hline Abbildung 9-5 Verteilung der Kerntypen & 72 \\
\hline
\end{tabular}

\begin{tabular}{|ll}
\hline Abbildung 10-1 Wichtigkeit der Führungstätigkeiten & 75 \\
\hline Abbildung 10-2 Wichtigkeit der Fuhrungstatigkeiten im Detail & 76 \\
\hline
\end{tabular}

\begin{tabular}{lr}
\hline Abbildung 10-2 Wichtigkeit der Führungstätigkeiten im Detail & 76 \\
\hline
\end{tabular}

\begin{tabular}{lr}
\hline Abbildung 10-3 Zeitaufwand für Führungstätigkeiten & 79 \\
\hline Abbiden & 80 \\
\hline
\end{tabular}

\begin{tabular}{lr}
\hline Abbildung 10-4 Führungsverhalten & 80 \\
\hline
\end{tabular}

\begin{tabular}{|lr|}
\hline Abbildung 11-1 Persönliche Erfolgsbeurteilung & 82 \\
\hline Abbidung 11-2 Abonnements-Verkäufe & 84 \\
\hline
\end{tabular}

\begin{tabular}{|lr|}
\hline Abbildung 11-2 Abonnements-Verkäufe & 84 \\
\hline Abbilung 11-3 Umfang der- & 86 \\
\hline
\end{tabular}

\begin{tabular}{|lr|}
\hline Abbildung 11-3 Umfang der Investitionen der letzten drei Jahre & 86 \\
\hline Abbilung 11-4 Perong & 87 \\
\hline
\end{tabular}

\begin{tabular}{lr}
\hline Abbildung 11-4 Persönliche Erfolgsbeurteilung & 87 \\
\hline Abbildung 11-5 Rentabe Center & 88 \\
\hline
\end{tabular}

\begin{tabular}{|lr|}
\hline Abbildung 11-5 Rentable Center nach Abonnementsverkäufen & 88 \\
\hline Abbildung & 90 \\
\hline
\end{tabular}

\begin{tabular}{lr}
\hline Abbildung 11-6 Erfolgsfaktoren für Mitgliederzahlen & 90 \\
\hline Abbildung 11-7 Erfolgsfaki & 91 \\
\hline
\end{tabular}

\begin{tabular}{lr}
\hline Abbildung 11-7 Erfolgsfaktoren für Auslastungsschwankungen & 91 \\
\hline Abbiddung $11-8$ Erfolgsfakn & 93 \\
\hline
\end{tabular}

\begin{tabular}{|lr|}
\hline Abbildung 11-8 Erfolgsfaktoren für Kundenzufriedenheit & 93 \\
\hline Abbldung 11-9 Erfolgsfakt & 94 \\
\hline
\end{tabular}

\begin{tabular}{lr}
\hline Abbildung 11-9 Erfolgsfaktoren für Gewinn & 94 \\
\hline
\end{tabular} 


\begin{tabular}{|ll|}
\hline Abbildung 12-1 Konkurrenzdruck & 100 \\
\hline Abbildung 12-2 Erfolgsfaktoren aus Kundensicht & 102 \\
\hline Abbildung 12-3 Erfüllung der Kundenanforderungen & 105 \\
\hline Abbildung 12-4 Wichtigkeit der Faktoren und Erfüllung durch das Center & 106 \\
\hline
\end{tabular}

\begin{tabular}{|ll|}
\hline Abbildung 13-1 Zeitaufwand pro Woche für Planen/Koordinieren & 110 \\
\hline Abbildung 13-2 Zeitaufwand pro Woche für Administration & 110 \\
\hline Abbildung 13-3 Zeitaufwand pro Woche für Motivieren & 112 \\
\hline Abbildung 13-4 Zeitaufwand pro Woche für Akquisition/Marketing & 112 \\
\hline Abbildung 13-5 Zeitaufwand pro Woche für Planen/Koordinieren & 115 \\
\hline
\end{tabular}

\begin{tabular}{|lr|}
\hline Abbildung 18-1 Flächenverteilung Stadt - Land & 135 \\
\hline Abbildung 18-2 Kundenverteilung Stadt - Land & 136 \\
\hline Abbildung 18-3 Verteilung des Konkurrenzdruckes Stadt - Land & 138 \\
\hline Abbildung 18-4 Verteilung des Konkurrenzdruckes Gross - Klein & 142 \\
\hline
\end{tabular}

Abbildung 20-1 Zusammenfassung 153 


\section{Tabellenverzeichnis}

Tabelle 3-1 Der gute Coach 21

Tabelle 3-2 Der gute Manager 22

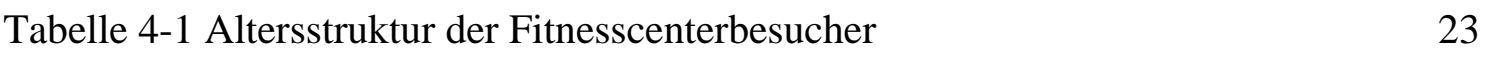

\begin{tabular}{|rr}
\hline Tabelle 4-2 Ausbildungsstand der Fitnesscenter-Besucher & 24 \\
\hline
\end{tabular}

\begin{tabular}{|lr}
\hline Tabelle 4-3 Hauptaktivität der Fitnesscenter-Kunden & 24 \\
\hline
\end{tabular}

Tabelle 4-4 Trainingshäufigkeit der Fitnesscenter-Besucher 25

Tabelle 4-5 Motivation für den Fitnesscenter-Besuch 25

\begin{tabular}{lr}
\hline Tabelle 4-6 Situative Variablen & 28 \\
\hline
\end{tabular}

\begin{tabular}{lr}
\hline Tabelle 8-1 Geographische Verteilung der Center & 59 \\
\hline
\end{tabular}

\begin{tabular}{lr}
\hline Tabelle 8-2 Flächenmässige Verteilung der Center & 61 \\
\hline
\end{tabular}

\begin{tabular}{|lr}
\hline Tabelle 8-3 Geschlechter-Verteilung der Center-Leitenden & 62 \\
\hline
\end{tabular}

Tabelle 9-1I Verteilung der vier Kerntypen 72

\begin{tabular}{lr}
\hline Tabelle 9-2 Vergleich der Verteilung der Kerntypen & 73 \\
\hline
\end{tabular}

Tabelle 10-1 Wichtigkeit der Führungstätigkeiten im Detail 75

\begin{tabular}{lr}
\hline Tabelle 10-2 Die wichtigsten Führungstätigkeiten & 77 \\
\hline
\end{tabular}

Tabelle 10-3 Führungstätigkeiten nach Fach- und Sozialkompetenz 78

\begin{tabular}{|rr}
\hline Tabelle 11-1 Persönliche Erfolgsbeurteilung & 82 \\
\hline
\end{tabular}

\begin{tabular}{|rr}
\hline Tabelle 11-2 Verteilung der CH-Center nach Fläche & 83 \\
\hline
\end{tabular}

\begin{tabular}{|rr}
\hline Tabelle 11-3 Abonnementsverkäufe & 84 \\
\hline
\end{tabular}

\begin{tabular}{|rr}
\hline Tabelle 11-4 Umfang der getätigten Investitionen & 86 \\
\hline
\end{tabular}

\begin{tabular}{|rr}
\hline Tabelle 11-5 Persönliche Erfolgsbeurteilung nach Bereichen & 87 \\
\hline
\end{tabular}

\begin{tabular}{|rr}
\hline Tabelle 11-6 Rentable Center nach Aboverkäufen & 88 \\
\hline
\end{tabular}

\begin{tabular}{lr}
\hline Tabelle 11-7 Effizienz: Fläche/Mitarbeiter & 95 \\
\hline
\end{tabular}

\begin{tabular}{lr}
\hline Tabelle 11-8 Vergleich Fläche/Mitarbeiter & 96 \\
\hline
\end{tabular}

Tabelle 11-9 Effizienz: Fläche/Mitglied 96

\begin{tabular}{|rr}
\hline Tabelle 11-10 Vergleich Fläche/Mitglied & 97 \\
\hline
\end{tabular}

\begin{tabular}{lr}
\hline Tabelle 11-11 Effizienz: Mitglied/Mitarbeiter & 97 \\
\hline
\end{tabular}

\begin{tabular}{lr}
\hline Tabelle 11-12 Vergleich Mitglieder/Mitarbeiter & 98 \\
\hline
\end{tabular}

\begin{tabular}{|lr|}
\hline Tabelle 12-1 Konkurrenzdruck & 100 \\
\hline Tabelle 12-2 Wichtigkeit von situativen Faktoren aus Kundensicht & 101 \\
\hline Tabelle 12-3 Hitliste der Erfolgsfaktoren aus Kundensicht & 102 \\
\hline Tabelle 12-4 Erfüllung der Kundenanforderungen & 105 \\
\hline Tabelle 12-5 Wichtigkeit für Kunden und deren Erfüllung & 106 \\
\hline
\end{tabular}

\begin{tabular}{|lr|}
\hline Tabelle 13-1 Führungsverhalten der Ausprägung E-I & 109 \\
\hline Tabelle 13-2 Zeitaufwand pro Woche für Planen/Koordinieren & 109 \\
\hline Tabelle 13-3 Zeitaufwand pro Woche für Administration & 110 \\
\hline Tabelle 13-4 Führungsverhalten der Ausprägung S-N & 111 \\
\hline
\end{tabular}




\begin{tabular}{|lr|}
\hline Tabelle 13-5 Zeitaufwand pro Woche für Motivieren/Bestärken & 111 \\
\hline Tabelle 13-6 Zeitaufwand pro Woche für Akquisition/Marketing & 112 \\
\hline Tabelle 13-7 Führungsverhalten der Ausprägung T-F & 113 \\
\hline Tabelle 13-8 Führungsverhalten der Ausprägung J-P & 114 \\
\hline Tabelle 13-9 Aufgewendete Zeit pro Woche für Planen/Koordinieren & 114 \\
\hline Tabelle 13-10 Extreme Zeitaufwand pro Führungstätigkeit & 116 \\
\hline
\end{tabular}

\begin{tabular}{|lr|}
\hline Tabelle 14-1 Erfolgs-Signifikanz der einzelnen Führungstätigkeiten I & 118 \\
\hline Tabelle 14-2 Erfolgs-Signifikanz der einzelnen Führungstätigkeiten II & 119 \\
\hline Tabelle 14-3 Extreme Führungsverhalten und Erfolg & 119 \\
\hline Tabelle 14-4 Führungstätigkeiten des sehr erfolgreichen Managers & 120 \\
\hline
\end{tabular}

\begin{tabular}{|c|c|}
\hline Tabelle 15-1 Rangliste der 16 MBTI-Typen nach Erfolg & 122 \\
\hline Tabelle 15-2 Rangliste der MBTI-Kerntypen nach Erfolg & 123 \\
\hline Tabelle 15-3 Rangliste der Kerntypen-Komponenten nach Erfolg & 123 \\
\hline Tabelle 15-4 Korrelationen der 4 MBTI-Ausprägungen mit Erfolg & 124 \\
\hline Tabelle 15-5 Persönlichkeitstypen von Sportstudierenden & 125 \\
\hline
\end{tabular}

\begin{tabular}{|lr|}
\hline Tabelle 16-1 Rangliste der situativen Erfolgsfaktoren & 129 \\
\hline Tabelle 16-2 Erfüllung der Erfolgsfaktoren & 130 \\
\hline
\end{tabular}

\begin{tabular}{l}
\hline Tabelle 17-1 Erfüllung der sit. Erfolgsfaktoren nach Typen \\
\begin{tabular}{|lr|}
\hline & 132 \\
\hline Tabelle 18-1 Verteilung Stadt-Land & 134 \\
\hline Tabelle 18-2 Flächenverteilung Stadt-Land & 135 \\
\hline Tabelle 18-3 Kundenverteilung Stadt-Land & 136 \\
\hline Tabelle 18-4 Verteilung des Konkurrenzdruckes Stadt - Land & 137 \\
\hline Tabelle 18-5 Personalvergleich Stadt - Land & 138 \\
\hline Tabelle 18-6 Wichtigkeitseinschätzung Führungsverhalten Stadt - Land & 139 \\
\hline Tabelle 18-7 Vergleich Führungstätigkeiten Stadt - Land & 139 \\
\hline Tabelle 18-8 Verteilung Gross - Klein & 141 \\
\hline Tabelle 18-9 Typenverteilung Gross - Klein & 141 \\
\hline Tabelle 18-10 Verteilung des Konkurrenzdruckes Gross - Klein & 142 \\
\hline Tabelle 18-11 Personalvergleich Gross - Klein & 143 \\
\hline Tabelle 18-12 Wichtigkeitseinschätzung Führungsverhalten Gross - Klein & 143 \\
\hline Tabelle 18-13 Vergleich Führungsverhalten Gross - Klein & 144 \\
\hline
\end{tabular}
\end{tabular}




\section{Einleitung}

\subsection{Problemstellung}

Kaum ein Bereich des Sports hat sich in den 80er und frühen 90er Jahren so explosiv entwickelt wie die Fitnessbranche. In Deutschland wurden in den 80er Jahren über 4000 Fitnesscenter eröffnet (BODYLIFE 1995). 1991 gehörten die 5000 Fitnesscenter mit ihren 60'000 Beschäftigten und einem Gesamtumsatz von 1.5 Mia. DM zum grössten Wachstumszweig der Dienstleistungsbranche (WEBER, SCHNIDER, KORTLÜKE \& HORAK 1994).

Dieses enorme Wachstum wurde begünstigt oder gar erst ermöglicht durch die markanten gesellschaftlichen Veränderungen der letzten 20 Jahre und die folgenden Faktoren:

- Entwicklung eines Fitnessbewusstseins in der Bevölkerung,

- Persönliche Fitness als Ausgleich und Mittel der Sinn- und Identitätsfindung,

- Stilisierung des perfekten Körpers,

- Zunehmende ökonomische Bedeutung der Fitnessbranche.

Das erwähnte Wachstum der Branche in den 80er und frühen 90er Jahren ist in den letzten drei Jahren abgeflacht. Fitness scheint im Produktelebenszyklus von der Wachstums- in die Reifephase überzugehen.

Neben dem Einbruch der zweistelligen Wachstumsraten weisen noch andere Faktoren darauf hin:

- Sättigung des Marktes, steigende Konkurrenz und damit echter Wettbewerb

- Unternehmensberater entdecken die Fitnessbranche

- Qualitätssicherung wird zum Thema

- Ausbildungsgänge für qualifiziertes Fachpersonal entstehen

- Die Sozialwissenschaft beginnt sich für das Phänomen „Fitness“ zu interessieren.

Zahlreiche Bücher sind in den letzten Jahren erschienen, die sich mit dem gesellschaftsund sozialpolitischen Wandel und der neuen Bedeutung des Fitnesssports auseinandersetzen (vgl. Kapitel 1.2 1 Wachstumsfaktoren der Fitnessbranche Seite 3 .

Die zunehmend ökonomische Bedeutung der Branche sowie der Anstieg an Beschäftigten schlug sich auch in einer Fülle von Untersuchungen und erscheinender Fachliteratur nieder. Die meisten Publikationen richten sich jedoch an den Fitnessleiter oder Trainer und geben Antworten auf fachlich-technische Fragen: Trainingsgestaltung, Fitnesstrends, Kundenbedürfnisse, Materialtests oder Geräteneuerungen. Der CenterManager selbst ist dabei nur am Rande (allenfalls noch im Bereich Fachkompetenz) ein Thema. Klassisches Managementwissen (strategisches Management, MarketingManagement, Betriebswirtschaft, etc.) hat sicher in der Fitnessbranche eine grosse Bedeutung. Angesichts der persönlichen und sozialen Atmosphäre eines Fitnesscenters spielen die Selbst- und Sozialkompetenz des Managers jedoch eine mindestens ebenso grosse Rolle wie die Fachkompetenz. 
Es ist deshalb erstaunlich, dass der Bereich Sozial- und Selbstkompetenz des Fitnesscenter-Managers, seine Einstellung zum Sport, seine Persönlichkeit und das damit zusammenhängende Führungsverhalten wenig untersucht wurden.

Diese Lücke will die vorliegende Arbeit schliessen.

Die empirische Untersuchung beschränkt sich dabei auf die Schweizer Fitnesscenter, die über das Qualitop-Gütesiegel verfügen.

Die schweizerischen Fitnesscenter befinden sich momentan in einem Prozess der Qualitätssicherung. Mit der Änderung des Schweizerischen Krankenversicherungsgesetzes (KVG) wurden die Krankenkassen verpflichtet, einen Teil Ihrer Gelder für Präventionsmassnahmen freizustellen. Eine Massnahme unter vielen war, den Besuch eines Fitnesscenters als Präventionsleistung der Versicherten zu anerkennen und im Sinne eines „Präventionsbonus“ mit bis zu Fr. 200.- pro Jahr zu subventionieren. Um eine gewisse Präventions-Qualität der Fitnesscenter sicherzustellen, haben sich die grössten Schweizer Krankenversicherungen zusammengeschlossen und das Gütesiegel "Qualitop“ lanciert, das nach Erfüllung gewisser Kriterien an die Fitness-Center abgegeben wird. Der Präventionsbonus wird nur mehr Versicherten gewährt, die in Qualitop-zertifizierten Centern trainieren.

Das Qualitop-Zertifikat orientiert sich an infrastrukturellen und personellen Kriterien. Die Frage des Managements wird bewusst ausgeklammert, weil im Fitnesscenterbereich noch zuwenig gesicherte Erkenntnisse über den Zusammenhang von Management und Erfolg bestehen.

Dazu will diese Arbeit erste Zahlen liefern.

Folgende praktische Fragen stehen dabei im Zentrum:

- Gibt es Zusammenhänge zwischen Persönlichkeit, Sportverständnis, Führungsverhalten und dem Erfolg eines Fitnesscenter-Managers?

- Wenn ja, welche Kriterien oder Faktoren (einzeln oder in Kombination) erhöhen die Chance des Erfolgs?

- Lässt sich ein charakteristisches Persönlichkeits- oder Führungsprofil des erfolgreichen und des nicht erfolgreichen Fitnesscenter-Managers zeichnen?

Ein solches Profil wäre einerseits für Qualitop eine wertvolle Ergänzung zu den bestehenden Zertifikats-Kriterien, andererseits könnte es von Consulting-Firmen im Fitnessbereich als Ausgangslage für eine Defizitanalyse mit entsprechenden CoachingMassnahmen von Fitnesscenter-Führungskräften verwendet werden.

Auf folgende wissenschaftliche Fragen sollen Antworten gegeben werden:

- Sind die Zusammenhänge von Persönlichkeit, Führungsverhalten und Erfolg auf andere Branchen übertragbar und decken sich die Anforderungen an Fitnesscentermanager mit den Anforderungen anderer Dienstleistungsbranchen?

- Wirken sich gesellschaftliche Veränderungan auf das Anforderungsprofil von Fitnesscentermanagern aus? 
Diese Betrachtungen erlauben es, die Erkenntnisse in einen grösseren Zusammenhang $\mathrm{zu}$ stellen und auf andere Dienstleistungsbetriebe und andere Management-Bereiche zu übertragen.

\subsection{Wachstumsfaktoren der Fitnessbranche}

Die in der Einleitung beschriebenen Faktoren, die zum rasanten Wachstum der Fitnessbranche geführt haben, werden im folgenden einzeln beschrieben und genauer analysiert.

\subsubsection{Entwicklung des Fitnessbewusstseins in der Bevölkerung}

Bereits in den 20er Jahren gab es erste Bodybuilding-Studios, von einem eigentlichen Fitnessbewusstsein in der Bevölkerung spricht FRIEDBERG (1984, S. 35) aber erst im Zusammenhang mit den Olympischen Sommerspielen 1972, als der unbekannte Amerikaner Frank Shorter die Goldmedaille im Marathon gewann. Dies inspirierte Tausende von gewöhnlichen Amerikanern

„to jog on the highways and byways in search of the rewards of staying fit."

Die Fitnesswelle dehnte sich in den mittleren 70er Jahren weiter aus, unter anderem auch weil bekannt wurde, dass Dr. COOPER (1968) aerobics entdeckt hatte - ein Trainingskonzept mit starker körperlicher Betätigung und dem Ziel von erhöhtem Sauerstoffverbrauch - und den gesundheitlichen Nutzen von körperlicher Fitness nachgewiesen hatte. Diese Entdeckungen wurden weiter gestützt durch FIXX (1977) und zusätzliche medizinische Studien (FRIEDBERG 1984, PAFFENBARGER 1989, BLAIR 1989 ).

Jane FONDA (1984) übernahm mitte der achtziger Jahre den Begriff der Cooperschen Aerobics für ihre Fitnessübungen und verhalf damit der Fitnessbewegung zu einem weiteren Popularitätsschub und neben der Strasse (Jogging) zu einem neuen Ausübungsort: das Zuhause (Videokassetten mit den Aerobic-Übungen) oder eben das Fitnesscenter mit den Aerobic-Instruktoren. Noch heute gehört Aerobic gehört zum Standardangebot eines grösseren Fitnesscenters.

Gegen Ende der achtziger Jahre setzte sich die Erkenntnis durch, dass Aerobics nicht die einzige Komponente eines Fitness-Programms sein sollte ( PATRAY \& CORTESE 1988, RIPPE 1989). Als ebenso wichtig wurden angesehen: Ernährung, Stresskontrolle, Selbstmanagement und der Zusammenhang von Fitness mit Selbstvertrauen und Selbstwertgefühl. Dieses breitere Fitnessverständnis (vgl. Kapitel 2.1, Begriffe, Seite 7 ) hat auch heute noch Bestand und ist Grundlage der vorliegenden Arbeit.

\subsubsection{Persönliche Fitness als Mittel der Sinn- und Identitätsfindung}

Wie das Fitnessverständnis haben sich in vielen westeuropäischen Ländern in den achtziger und neunziger Jahren auch die traditionellen sozialen und gesellschaftlichen 
Normen gewandelt. Dieser sogenannte „Fahrstuhl-Effekt“ (STELTER 1996) wird durch BECK (1986, S. 115) wie folgt beschrieben:

„Die ,Klassengesellschaft' wird insgesamt eine Etage höher gefahren. Es gibt - bei allen sich einpendelnden und durchgehaltenen Ungleichheiten - ein kollektives Mehr an Einkommen, Bildung, Mobilität, Recht, Wissenschaft, Massenkonsum. In der Konsequenz werden subkulturelle Klassenidentitäten und -verbindungen ausgedünnt oder aufgelöst. Gleichzeitig wird ein Prozess der Individualisierung und Diversifizierung von Lebenslagen und Lebensstilen in Gang gesetzt, der das Hierarchiemodell sozialer Klassen und Schichten unterläuft und in seinem Wirklichkeitsgrad in Frage stellt.“

Die traditionellen Handlungs- und Lebensstrategien wie Familie, soziale Netzwerke, Religion oder Patriotismus werden grundsätzlich in Frage gestellt. Das Individuum muss sich seine Identität und den Sinn des Lebens neu aushandeln.

Zusätzlich führt die zunehmende technisierte Welt zu einem Verlust an natürlichen, echten, sinnhaften und sinnvollen Erlebnissen (SCHMID 1997).

Dies führt gemäss RITTNER (1986) zu einer Renaissance des Körpers als Möglichkeit der Identitätsentwicklung. Der Körper verhilft mit seinen Symptomen und Empfindungen zu Situationen, die als intensiv, echt, sinnhaft, sinnvoll und ganzheitlich wahrgenommen werden.

Der eigene Körper kompensiert damit den Authenzitäts- und Identitätsverlust und wird zu einer Orientierungsinsel im täglichen Wandel der Werte:

„Versunken in ihre Aktivität und in die Auseinandersetzung und Verwirklichung mit einem unablässig Symptome und Signale sendenden, sinnhaft interpretierenden Körper blenden die Subjekte die Dissonanzen und Inkongruenzen eines lästigen Alltags und beruflicher wie familiärer Probleme aus. Bewirkt und genossen werden - wenn auch nur auf Zeit - ein Mikrokosmos der Person-Umwelt-Übereinstimmung und ein Biotop individueller Authenzität. Statt Stress ergibt sich Konsonanz.“ (RITTNER 1986, S. 24).

Im Leiberleben nimmt sich also das Subjekt als selbstwirksam wahr und kann so sein Selbstbild und Selbstvertrauen aufbauen.

Die obigen Ausführungen zeigen, wie der gesellschaftlich und soziale Wandel der letzten fünfzehn Jahre das Sporttreiben im allgemeinen begünstigt hat. Wie lässt sich jedoch der überproportionale Anstieg im kommerziellen Fitnesssport erklären?

Die verloren gegangene Selbstkontrolle oder internale Kontrolle (ROTTER, 1972) - „,verstärkte Erwartungshaltungen des Individuums darüber, ob es durch eigenes Verhalten Verstärker und wichtige Ereignisse in seinem Leben beeinflussen kann“ kann gerade im Fitnesssport sehr eindrücklich wiedergewonnen werden. STELTER (1996) konnte in seiner empirischen Studie über Selbstkonzept und Identität durch Sport nachweisen, dass der Begriff „Selbstkontrolle“ gerade bei Besuchern von Fitnesscentern signifikant häufiger als bei anderen Sportarten als Trainingsmotivation angegeben wird. Er unterscheidet dabei drei Kategorien. 
1. Leibwahrnehmungen als Basis der Selbst-Kontrolle.

Die SportlerInnen erleben Gefühle wie ,gesunde Müdigkeit“ als durch das eigene

Selbst kontrollierbar.

2. Erleben von Körperkontrolle vermittelt Selbstkontrolle

Über das Führen von Trainingstagebüchern und Trainingskontrollen erleben die SportlerInnen ein Gefühl von Körperkontrolle, das ihnen wiederum ein Gefühl von Selbstkontrolle vermittelt.

3. Trainingsrhythmus strukturiert den Alltag und vermittelt Gefühl von allgemeiner Selbstkontrolle.

Die sportliche Aktivität im Fitnesscenter wird als strukturierender Faktor im Alltag erlebt und vermittelt so das Gefühl von allgemeiner Selbstkontrolle.

\section{Abbildung 1-1 Kategorien der Selbstkontrolle von Fitnesscenter-Besuchern} (STELTER, 1996)

\subsubsection{Die Stilisierung des perfekten Körpers}

Gutes Aussehen und der perfekte Body wurden in den achziger und frühen neunziger Jahren zum Synonym für Erfolg. Aussehen wird deshalb in einigen empirischen Studien dieser Zeit (RITTNER \& MRAZEK 1986, DIETRICH, HEINEMANN \& SCHUBERT 1990) als Hauptargument von sportlicher Betätigung genannt. Dies wurde jedoch bereits damals mit anderen Studien widerlegt, welche "Gesundheit" als das wichtigste Argument für Sporttreiben ( SAMPLE INSTITUT 1984, KLAUDI KLAUSEN 1989) angaben.

$\mathrm{Zu}$ diesen Widersprüchen in der empirischen Forschung kann folgende Tatsache als Erklärungsversuch beigezogen werden: je nach Center kann die Hauptausrichtung ganz unterschiedlich sein. Ein Center, das im Stile der alten Body-Building-Keller eingerichtet ist, zieht eher eine Kundschaft an, die ihre Trainingsziele im Bereich von Kraft und Aussehen hat. Ein Center, das neben dem eigentlichen Krafttraining bewusst auch Wert auf Herz-/Kreislauftraining und Stretching legt oder sogar zusätzliche Dienstleistungen im Gesundheitsbereich anbietet (z.B. Massage, Entspannungstraining, Ernährungsberatung, Zusammenarbeit mit Physiotherapien) ziehen ihrerseits wieder eher Kunden an, bei denen Gesundheit der Hauptgrund ihres Besuches ist. Die unterschiedlichen Ergebnisse der verschiedenen Studien sind darum möglicherweise durch eine unterschiedliche Auswahl der befragten Center zu erklären.

Auch heute gibt es noch die unterschiedlich ausgerichteten Center. Die klassischen Kraftkeller sind aber eher am Aussterben (unter anderem auch dadurch, dass sie von QUALITOP nicht akzeptiert und damit von den Krankenkassen nicht subventioniert werden, vgl. Kapitel 4.3 Qualitop Seite 26. 
Noch heute wird in der Werbung der perfekte Körper von Models zur Darstellung von erfolgreichen Personen verwendet und trainierte Körper verhilft dem Fernsehen auch zu Einschaltquoten:

„Die gesellschaftliche Modernisierung hat eine Intellektualisierung mit sich gebracht, die den Körper immer mehr in den Hintergrund gerückt hat. Der Sport setzt durch eine konkrete, anschauliche Körperästhetik den Gegensatz zur Abstraktheit der modernen Gesellschaft. Der Sportzuschauer geniesst eine Ästhetik der Perfektion körperlicher Abläufe, aber auch eine Ästhetik des Kampfes und des Siegeswillens. Diese Formen der Ästhetik sind für den Zuschauer als Konsumenten allgemein verständlich und bedürfen keinerlei intellektueller Anstrengungen." (Werner, 1997, Seite 63)

Als Grund für den Fitnesscenterbesuch ist aber mittlerweile Aussehen und Figurerhaltung (14\% bei Frauen, $12 \%$ bei Männern) weit hinter Gesundheit (56\% der Frauen, 52\% der Männer) und Rehabilitation (18\% der Frauen und Männer) auf Platz drei abgerutscht, wie die breit angelegte Untersuchung der Schweizerischen Beratungsstelle für Unfallverhütung bfu bei 15'000 Fitnesscenterbesuchern (BFS 1998) zeigte.

\subsection{Vorgehen}

Im theoretischen Teil müssen zuerst die verschiedenen später zu verwendenden Begriffe definiert, erklärt und abgegrenzt werden. Anschliessend soll ein Überblick über den Stand der Forschung im Bereich Erfolg und Management gegeben werden und ein für unsere Problemstellung nützliches Modell gefunden werden. Die verschiedenen Bestandteile des gefundenen Modells werden danach einzeln betrachtet und mögliche Erhebungsinstrumente oder Tests diskutiert und verglichen.

Aufgrund dieser Erkenntnisse wird im empirischen Teil ein Untersuchungsinstrument entworfen und eingesetzt, um die im Kapitel 1 erwähnten Fragen zu beantworten. 


\section{Begriffe und Abgrenzungen}

\subsection{Begriffe}

Die Begriffe Persönlichkeit, Sportverständnis, Führungsverhalten und Erfolg werden zu Beginn der einzelnen Kapitel behandelt.

\section{Fitness}

Der Fitness-Begriff hat sich im Laufe der Jahre entwickelt.

In den Anfängen des Fitnessbooms in Amerika in den 70er Jahren stand vor allem der körperliche Aspekt in Zusammenhang mit einer athletischen Leistung im Vordergrund. So bezeichneten GISOLFI \& LAMB (1989) Fitness als eine körperliche Fähigkeit, die sich vor allem auf die Erbringung einer athletischen Leistung auswirkt.

In Zusammenhang mit den verschiedenen Studien über die Auswirkungen von Ausdauersport auf Herz-/Kreislauferkrankungen (vgl. Kapitel 1.2.1 Entwicklung des Fitnessbewusstseins in der Bevölkerung. Seite 3] wurde in den frühen 80er Jahren eher der Bezug zur Gesundheit betont:

"Fitness is an ability to perform daily activities with vigor and is characterized by traits and capacities that are associated with low risk of premature development of hypokinetic diseases (i.e. those associated with physical inactivity)." (BOUCHARD et al., 1990, S. 123).

Mit der Veränderung des Körperbildes und dem Entdecken der sinnstiftenden Komponente von sportlichen Aktivitäten in der Freizeit (vgl. Kapitel 1.2.2 Persönliche Fitness als Mittel der Sinn- und Identitätsfindung Seite 3] wurde Fitness eher als ganzheitliches „Gerüstet-Sein für Beruf und Freizeit“ definiert:

"Being fit means the ability to carry out daily tasks with vigor and alertness, without undue fatigue, and with an ample energy to enjoy leisure time pursuits and to meet unforseen emergencies." (PETRAY \& CORTESE 1988, S. 19).

Im deutschen Sprachgebiet definiert SCHNABEL (1993, S. 299) Fitness als

„Zustand guter körperlicher und psychischer Leistungsvoraussetzungen für die Bewältigung einer bestimmten Tätigkeit bzw. Situation."

Er betont, dass Fitness eine wesentliche Zielkomponente des Freizeit- und Breitensports darstellt, dass sie sich aber auch auf alltägliche Situationen bezieht. Es gebe in dem Sinn keinen universellen Fitnessbegriff, sondern man sei „fit für eine bestimmte Aufgabe“. (SCHNABEL 1993).

KAYSER (1987) beschreibt ähnlich allgemein wie PETRAY \& CORTESE (1988) Fitness als Lebenstauglichkeit des Menschen sowie dessen aktuelle Eignung für beabsichtigte Handlungen.

Je nach Situation oder Bezug lassen sich gemäss der untenstehenden Tabelle verschiedenen Fitnesskonzepte unterscheiden. 
Verschiedene Fitnesskonzepte

- Prävention gegenüber Krankheiten

- Retardation des Alterungsprozesses

- Erhaltung und Steigerung der beruflichen Eignung

- Motorische Optimierung

- Rekreation und körperliches Wohlbefinden

- Beitrag zur Selbstverwirklichung (z.B. Freude, Lust, emotionale Stabilität, Schönheit)

- Beitrag zum Kontakt mit der Umwelt (z.B. Geselligkeit, Anerkennung durch andere, Naturerlebnisse)

Abbildung 2-1 Fitnesskonzepte (nach KAYSER, 1997)

Die aufgeführten Konzepte sind in konkreten Programmen oft miteinander verbunden und so kommt als zusätzliche Erschwerung eines Definitionsversuchs von Fitness dazu, dass die Begriffe Fitness und Gesundheit (,vollständiges körperliches, geistiges und soziales Wohlbefinden“ gemäss WHO) zu Synonymen werden. Dies wird auch in der amerikanischen Literatur betont (GLASSNER, 1989).

Als gemeinsamer Nenner der verschiedenen erwähnten Definitionen soll für diese Arbeit der folgende Fitnessbegriff verwendet werden:

ein zur Bewältigung einer Berufs-, Alltags- oder Freizeitsituation guter körperlicher Zustand, der ein geistiges und soziales Wohlbefinden mit sich bringt.

Es ist dabei jedoch nicht nur das Wohlbefinden dessen wichtig, der das Fitnesstraining betreibt, sondern gemäss MERTENS \& VAHL (1995) auch und vor allem, wie er eben mit diesem Wohlbefinden auf andere wirkt. Es komme nach ihnen darauf an, wie sich dieser Zustand für den zweckgerichteten Einsatz im Rahmen bestimmter gesellschaftlicher Erwartungen nutzvoll einsetzen lässt.

Wenn wir noch etwas in die Zukunft blicken und uns den Fitnessbegriff im 21. Jahrhundert ansehen, so werden dannzumal vor allem Werte wie Selbstverantwortung und Selbstverwirklichung durch ganzheitliches Tun und Sein im Vordergrund stehen oder mit den Worten von HERRON (1992, S. 124):

"Authorities writing in the field suggest that the twenty-first century definition of fitness will focus on self-directed awareness and action aimed toward integrating physical, emotional, social and spiritual life to achieve optimal performance within our environment." 


\section{Das Fitnesscenter}

Unter Fitnesscenter werden in dieser Arbeit Fitnesscenter verstanden, die mit dem Qualitätslabel „QUALITOP“ ausgezeichnet sind oder die Bedingungen dazu erfüllen:

- Angebot des Centers. Möglichkeit für unverbindliches Probetraining, Abklärung des Gesundheitszustandes vor dem Trainingsbeginn

- Vertrag: Möglichkeit zur Vertragsunterbrechung, automatisch Erneuerung nur nach schriftlicher Ankündigung

- Inbegriffene Leistungen: Eintrittstest, individueller Trainingsplan

- Infrastruktur für das Krafttraining: Kraftgeräte für gewisse Muskelgruppen, Minimalzahl an Geräten pro Mitglieder

- Infrastruktur für das Ausdauertraining: mindestens zwei Gerätevarianten mit Möglichkeiten für herzkontrolliertes Training

- Infrastruktur für Beweglichkeitstraining: spezieller Bereich mit Hinweistafeln

- Infrastruktur für Aerobic und Gymnastik: Minimalgrössen der Räumlichkeiten pro Mtglied, unterschiedliche Level

- Betreuung und Personal: Minimalanforderungen im Bereich Ausbildung der Mitarbeitenden, Minimalzahl an Mitarbeitenden pro Mitglied

- Zusatzangebote: Verkauf nur von BAG-zugelassenen Stärkungspräparaten

- Betriebsführung: Kontakt zu einem Arzt und/oder Physiotherapeuten, Reiningungskonzept

(detaillierte Bedingungen, siehe QUALITOP, 1997).

\section{Der Manager}

Der Begriff „Manager“ ist längst zu einem umgangssprachlichen Begriff geworden. Um so wichtiger ist folglich eine klare Eingrenzung für diese Arbeit.

In der Psychologie wird das Thema „Manager“ unter dem Aspekt der Person behandelt und den Begriffen „Führer“ oder „Vorgesetzter“ zugeordnet. Dies ergibt zum Beispiel folgende Definition:

„Führung ist die bewusste und zielbezogene Beeinflussung von Menschen.“ (VON ROSENSTIEL, MOLT \& RÜTTINGER, 1988, S. 23)

Diese Sicht vernachlässigt, dass ein Manager nicht nur Menschen führt, sondern auch andere Ressourcen als menschliche Arbeit optimierend einsetzen muss. Die Betriebswirtschaftslehre liefert hier ein abgerundeteres, jedoch auch komplexeres Bild des Managers:

„Führung ist die Gesamtheit der Institutionen, Prozesse und Instrumente, welche im Rahmen der Problemlösung durch eine Personengemeinschaft (mit komplexen zwischenmenschlichen Beziehungen) der Willensbildung (Planung und Entscheidung) und der Willensdurchsetzung (Anordnung und Kontrolle) dient." (RÜHLI, 1985, S.28)

„Führungskräfte sind Personen, die dispositiv tätig sind und in nennenswertem Umfang personelle, sachliche und finanzielle Verantwortung tragen und entsprechende Kompetenzen haben." (ATAG/SHZ/IHA, 1988, S. 15) 
„Manager sind Personen, die eine Führungs-Funktion ausüben und die damit verbundene Verantwortung, namentlich in personeller, sachlicher und finanzieller Hinsicht zu tragen haben.“ (ISELE, 1991, S.15)

Wesentliches Unterscheidungsmerkmal zwischen Managern, Führungskräften oder Kader (diese Begriffe werden in dieser Arbeit synonym verwendet) und den übrigen Mitarbeitenden des Unternehmens ist also die aus der Führungsaufgabe resultierende Anordnungsbefugnis mit der damit verbundenen Verantwortung. Als weitere Unterscheiungsmerkmale werden genannt (ATAG/SHZ/IHA, 1988, S. 15):

- Wichtige Stellung im Unternehmen (Schlüssel- Vertrauensposition, Spezialist, Experte)

- Besondere Vertrags- und/oder Gehaltsregelung

- Mitwirkung bei der Erfüllung unternehmerischer Aufgaben (mit Einfluss auf den Geschäftsgang)

- Qualifizierte Ausbildung, hohes Qualifikationsniveau

Diese betriebswirtschaftlichen Definitionen sind für die vorliegende Arbeit besser, da sie beide Facetten des Führungsprozeses, nämlich die psychologische, aber auch die instrumentell-operative, beinhalten.

\section{Das Unternehmen}

Ein Unternehmen kann gesehen werden als

„ein offenes, dynamisches, komplexes, teilweise probabilistisches, teilweise autonomes, zielgerichtetes und zielsuchendes produktives soziales System.“ (RÜHLI 1985, S. 15).

Diese Definition deckt den Begriff der Unternehmung als soziales und betriebswirtschaftliches Gefüge umfassend $a b$ und passt zu unserer oben verwendeten, zweifachen Definition von Führung.

In der Definition von Managern wurde die Verantwortung hervorgehoben, die mit der Führungsfunktion eindrehet. Man spricht in diesem Zusammenhang von „Responsibility Center":

"an organization unit headed by a responsible manager" (ANTHONY, DEARDEN \& BEDFORD 1989, S. 26).

Je nach Verantwortungsbereich kann der Grad der Verantwortlichkeit variieren. In der Literatur werden verschiedene Verantwortlichkeitsbereiche unterschieden, die jedoch je nach Autor anders definiert werden. Eine weit verbreitete Klassifikation ist die folgende von DEAKIN \& MAHER (1987), die vier Typen von Verantwortungsbereichen unterscheidet:

- Cost Center

- Revenue Center

- Profit Center

- Investment Center 
Grundsätzlich gilt für alle Bereiche, dass über bestimmte Ressourcen (Input) verfügt, diese Ressourcen in einer bestimmten Weise bearbeitet und dadurch ein Resultat (Output) erzielt, das je nach Kriterien als erfolgreich oder nicht erfolgreich bewertet werden kann. Die Bearbeitung der Ressourcen richtet sich dabei nach den Kriterien der Effektivität und Effizienz (vergleiche 3.5 Trainerpersönlichkeit und Erfolg Seite 18).

\section{Cost Center}

Der Leiter eines Cost Centers ist verantwortlich für die Kosten der Leistungserbringung seines Bereichs. Die zur Verfügung stehenden Ressourcen sind meist gegeben, wie auch der zu leistende Output. Der Cost Center Manager ist also primär mit der Optimierung von Input und Output beschäftigt (Effizienz).

\section{Revenue Center}

Leiter von Revenue Center sind verantwortlich für die Vermarktung und den Verkauf von Produkten und den damit erzielten Rückfluss an Mitteln (revenue). Sie haben in der Regel keinen Einfluss auf die Herstellungskosten und sind deshalb nur für die erzielten Erträge verantwortlich. Im Sinne der Output-Optimierung sind sie ebenfalls vor allem Effizienz-orientiert.

\section{Profit Center}

Wenn der Manager neben der absatzbezogenen Kompetenzen (Revenue Center) auch noch über umfassende produktionsbezogene Kompetenzen, so spricht man von einem Profit Center. Seine Zielorientierung ist dann ein vorgegebene Form von finanziellem Gewinn, oft ergänzt mit nichtfinanziellen Zielen wie z.B. Marktanteil.

Der Profit Center Manager ist sowohl um Effizienz, aber auch um Effektivität besorgt.

\section{Investment Center}

Manager von Investment Center sind mit dem höchstmöglichen Grad an Kompetenzen, aber auch Verantwortung ausgerüstet. Neben Input- und Output-Verantwortung entscheiden sie auch über längerfristige Investitionen und damit über strategische Belange des Unternehmens.

Es ist nicht möglich, die Fitnesscenter-Manager klar der einen oder anderen Kategorie zuzuordnen. Der Manager kann der Inhaber des Centers sein und hat damit die Funktion des Leiters eines Investment-Centers mit dem grösstmöglichen Grad an Kompetenzen, aber auch Verantwortung. Der Manager kann jedoch auch ,nur“ Geschäftsführer eines Franchising-Betriebes sein und damit nur die Verantwortung für das operative Tagesgeschäft haben, ohne sich um die strategische Ausrichtung des Unternehmens, das Marketing oder grössere Investitionen kümmern zu müssen. In diesem Falle ist er eher Leiter eines Cost oder Revenue Centers.

Diese klassische betriebswirtschaftliche Aufteilung hilft uns hier also nicht sehr viel weiter.

Es kann immerhin festgestellt werden, dass der Leiter eines Fitnesscenters grundsätzlich einen recht bis sehr grossen Freiheitsgrad in seinen Entscheidungen hat (vergleiche „starke“ und „schwache“ Situationen von ICKES, 1982), womit sich die Persönlichkeit des Managers stark auf das entsprechende Verhalten auswirkt (vergleiche 3.1. Persönlichkeit und Erfolg, Seite 14. 


\subsection{Abgrenzungen}

\section{Unternehmen und Fitnesscenter}

Damit die Untersuchungsergebnisse miteinander verglichen werden können, muss der Begriff der Unternehmung noch weiter eingeschränkt werden. Es kann darauf verzichtet werden, eigene Kriterien $\mathrm{zu}$ formulieren, da QUALITOP solche Kriterien bereits festgelegt hat (vergleiche Seite 9). Da alle in der Untersuchung befragten Center QUALITOP-zertifiziert sind und damit alle QAULITOP-Kriterien erfüllen, können diese Kriterien übernommen werden.

\section{Manager und Fitnesscenter-Manager}

Grundsätzlich unterscheiden sich die Führungsaufgaben des Fitnesscenter-Managers wenig von Führungsaufgaben in anderen Branchen. In kleinen Betrieben ist der Geschäftsleiter jedoch oftmals auch noch als Trainer tätig. Da unsere Untersuchung auch kleinere Betriebe umfasst, ist dieser Traineraspekt auch noch zu berücksichtigen.

In grösseren Center wird der Geschätsführer oder Manager sich fast ausschliesslich auf das Führen des Betriebes konzentrieren und nicht auch noch TrainingsleitungsFunktionen übernehmen können. Viele Fitnesscenterleiter sind jedoch als Trainer gross geworden und haben sich sukzessive ins Management eines Betriebes eingearbeitet.

Im Bereich „Trainerpersönlichkeit und Erfolg“ bestehen bereits viele Untersuchungen, die in einem speziellen Unterkapitel vorgestellt werden (vergleiche Kapitel 3.5 Trainerpersönlichkeit und Erfolg. Seite 18.

\section{Gewinnorientierte und Non-Profit-Organisationen}

Eine oftmals in den Betriebswissenschaften verwendete Unterscheidung ist diejenige von gewinn-orientierten Unternehmen und Non-Profit-Organisationen. BADELT (1999) schlägt in Anlehnung an SALOMON \& ANHEIER (1996) fünf Kriterien vor, die eine NPO charakterisieren:

\section{Mindestmass an formaler Organisation}

Explizit wird keine besondere Rechtsform verlangt, hingegen werden spontane oder lediglich auf einen einzigen, temporären Anlass bezogene Aktivitäten nicht als NPOs angesehen.

2. Private, nicht-staatliche Organisationen

Dies schliesst jedoch eine teilweise Finanzierung durch den Staat nicht aus.

3. Keine Gewinn- oder Überschussausschüttung an Eigentümer oder Mitglieder Das heisst nicht, dass NPOs keine Gewinne erwirtschaften dürfen. Der Gewinn muss aber in der Organisation verbleiben und für den Unternehmenszweck verwendet werden.

4. Minimum an Selbstverwaltung und Entscheidungsautonomie

Die wichtigsten Entscheidungen können im Unternehmen gefällt werden. Eine Aussenkontrolle findet nur parziell statt.

\section{Freiwilligkeit}

Darunter werden ehrenamtliche Tätigkeiten von Mitgliedern oder Funktionären, aber auch freiwillige Mitgliedschaften und Spenden verstanden. 
Keines der Kriterien ist absolut trennscharf. Obwohl dies im Hinblick auf die Verwendung des Begriffes hilfreich wäre, entspricht die Unschärfe der Grenzen sehr gut den auch in der Realität unscharfen Trennlinien zwischen NPOs und gewinnorientierten Organisationen.

Auch bei den Fitnesscentern ist die Unterscheidung nicht immer ganz klar zu treffen. Gehört das Unternehmen einer Privatperson, so wird es diese in der Regel gewinnorientiert führen. Gehört das Fitnesscenter jedoch einer Firma, die für ihre Mitarbeiter ein eigenes Fitnesscenter betreiben will, so steht dort vor allem die Dienstleistung an den Mitarbeitenden und weniger der Gewinn im Vordergrund. Diese Art von Fitnesscenter wird aber mit der vorliegenden Untersuchung gar nicht erfasst.

Aus diesem Grund wird in dieser Arbeit davon ausgegangen, dass die Fitnesscenter gewinnorientiert geführt werden.

\section{Individualleistung und Gruppenleistung}

Im Zentrum unserer Untersuchung steht der Fintnesscenter-Manager und sein Beitrag zum Erfolg eines Fitnesscenters. Die Individualleistung des Managers steht jedoch immer in Wechselwirkung mit der Leistung der Gruppe oder des Teams, dem der Manager vorsteht. Die Koordination der verschiedenen Einzelleistungen der Teammitglieder zu einer Kollektivleistung liegt jedoch in der Hand des Managers. Deshalb wird für diese Arbeit vereinfachend nicht berücksichtigt, wie gross der Anteil der einzelnen Teammitglieder am Gesamterfolg ist. Der Erfolg (vergleiche Kapitel 7 Der Führungserfolg Seite 40 des Fitnesscenters wird folglich als Individualleistung des verantwortlichen Managers gesehen.

Dies ist ein sehr grobe Vereinfachung, zumal der Stellenwert des Personals, wie die Untersuchungsergebnisse zeigen, in der Branche sehr hoch ist. Es hätte aber den Rahmen der Möglichkeiten dieser Arbeit gesprengt, das Personal auch noch in die Studie miteinzubeziehen. 


\section{Erfolg und Management: Stand der Forschung}

Wie schon eingangs erwähnt, hat die Diskussion um den Zusammenhang von Management und Erfolg in den Wirtschaftswissenschaften Tradition. Die folgende kurze Übersicht zeigt, wie sich die Forschung entwickelt hat und welche Modelle heute akzeptiert sind. Die Auflistung kann dabei keineswegs vollständig sein, sondern soll vor allem die Entwicklung mit einigen Meilensteinen aufzeigen.

\subsection{Persönlichkeit und Erfolg}

Ein erster Ansatz ging von einem Zusammenhang von Persönlichkeit und Erfolg aus und spiegelt sich in den ,great man“-Theorien von CARLYLE (1841) und GALTON (1869) über das Erreichen grosser Ziele. Diese monokausale Sicht des Zusammenhangs von Persönlichkeit und Erfolg wurde in der Vergangenheit mehrfach kritisiert (vgl. SARGES 1995, S. 1) und sogar widerlegt: STOGDILL (1948) und MANN (1959) kamen zum Schluss, dass keines der vielen untersuchten Persönlichkeitsmerkmale deutlich und konsistent über verschiedene Führungssituationen hinweg Führer und Nicht-Führer unterscheidet.

Damit wurde in den 60er und 70er Jahren mehrheitlich davon ausgegangen, dass Persönlichkeitsmerkmale als Prädiktoren für den Berufserfolg nicht recht tauglich sind. Im Bereich von Trainerpersönlichkeit und Erfolg konnten jedoch Zusammenhänge nachgewiesen werden (vergleiche Kapitel 3.5 Trainerpersönlichkeit und Erfolg, Seite 18. Diese Zusammenhänge konnten später in den 80er Jahren auch für andere Führungsbereiche gezeigt werden. Die wichtigsten Exponenten sind dabei GOUGH (1984) mit dem CPI, dem California Psychological Inventory, MCCRAE\& COSTA (1987) und GOLDBERG (1990) mit dem Fünf-Faktoren-Modell „The Big Five“.

\subsection{Situation und Erfolg}

Die Situation stand anschliessend im Mittelpunkt des Interessens. Es wurde festgestellt, dass die Situation in zweifacher Weise einflussnehmend ist: Zum einen gibt es einen direkten Einfluss auf den Erfolg: z.B. sehr gute Mitarbeiter oder Hochkonjunktur als positive Beeinflusser oder Weggang von schwer ersetzbaren Experten oder Wasserschaden mit vorübergehender Geschäftsschliessung als negative Beinflusser. Zum anderen gibt es den indirekten Einfluss auf den Erfolg über das Verhalten des Managers.

Diese Erkenntnisse führten zum Ansatz der Situationstheorien von FIEDLER (1967), HERSEY \& BLANCHARD (1977) und REDDIN (1981). Diese Situationstheorien gehen davon aus, dass die Situation eine dominante Variable für Führungserfolg darstellt. Die Situation, in welcher der Führer agiert, kann mehr oder weniger günstig für ihn sein, indem sie die Einflussnahme des Managers erleichtert oder überhaupt ermöglicht. 
Drei Faktoren werden dabei genannt, welche die Günstigkeit der Situation beeinflussen (FIEDLER, 1967, präzisiert nach CHELLADURAI \& KUGA, 1996):

- Führer-Geführte-Beziehung

- Problem- oder Aufgabenstruktur

- Machtstellung des Führers

Die Situation ist dabei weit zu verstehen: sie beinhaltet die Kultur des Landes und des Unternehmens, die Branche, die Art der Aufgaben, die Grösse der Arbeitsgruppe, die Qualifikation und Eigenart der Mitarbeiter, etc.

Sowohl JÄGER (1983) als auch später FIEDLER selbst (1987) weisen darauf hin, dass die Situation-Erfolg-Untersuchung nicht umhin kommt, die Person auch mit einzubeziehen - wie dies durch CHELLADURAI \& KUGA (1996) in ihrer Präzisierung auch gemacht wurde (die Führer-Geführte-Beziehung lässt sich ohne die Betrachtung der jeweiligen Persönlichkeiten kaum analysieren...).

Eine intensive Diskussion der Kontingenzmodelle der Führung in Bezug auf relevante situative Führungsvariablen findet sich bei HELLRIEGEL \& SLOCUM (1992).

\subsection{Verhalten und Erfolg}

Als nächster Ansatz wurde ein Zusammenhang von Verhaltens-Merkmalen und Führungserfolg postuliert und untersucht. Drei verschiedene Richtungen können dabei unterschieden werden (FLEISHMAN, ZACCARO \& MUMFORD, 1991):

- Beschreibung von charakteristischen Verhaltensmustern von Führungspersonen

- Fokussierung auf die situativen Faktoren des Führungsverhaltens

- und ein transaktionaler Ansatz des sozialen Austauschs von Führern und Geführten.

Die erzielten Ergebnisse der verhaltensorientierten Forschung gaben gemäss SARGES (1995) keine grosse Hilfe bei den Problemen der Selektion oder Beförderung von Führungspersönlichkeiten. Es setzte sich die Überzeugung durch, dass Verhalten einen Einfluss auf den Führungserfolg hat, jedoch isoliert zu wenig prognostische Relevanz für den Gebrauch in Personalabteilung hat. Spätestens bei der Frage des ,,person-job-fit“ war man wieder bei der Person angelangt.

\subsection{Person und Situation und Erfolg}

Also wurde die Frage nach der Persönlichkeit wieder aktuell und verschiedene Untersuchungen zeigten, dass der Schluss, Persönlichkeit könne nicht mit Erfolg assoziiert werden, ein vorschneller war. LORD, DE VADER und ALLIGER (1986, S. 407) reanalysierten die Studien von MANN (1959) und kamen zu folgendem Schluss:

"Personality traits are associated with leadership perceptions to a higher degree and more consistently than the popular literature indicates."

HOWARD \& BRAY (1988) konnten nachweisen, das Persönlichkeitsmerkmale von Managerkandidaten in der Lage waren, spätere Karrierestufen in überraschenderweise vorherzusagen. FLEISHMAN et al. (1991) fanden, dass kognitive Fähigkeiten und eine Reihe weiterer Persönlichkeitsmerkmale mit Organisationserfolg zusammenhängen. HOGAN (1991), BRANDSTÄTTER (1993) und KLINE (1993) bestätigen die 
Zusammenhänge von Persönlichkeit und Berufserfolg ebenfalls. Auch im Sport ist der Zusammenhang von Persönlichkeit und Erfolg ein Thema (siehe 3.5 Trainerpersönlichkeit und Erfolg, S. 18,

Mit diesen Funden setzte sich die Erkenntnis durch, dass die Manager-Persönlichkeit Voraussagen auf den Erfolg zulässt, dass jedoch noch andere Faktoren hineinspielen. Davon ausgehend und mit Anleihen in den oben beschriebenen Ansätzen entwickelte VON ROSENTIEL (1993) folgendes Führungs-Modell.

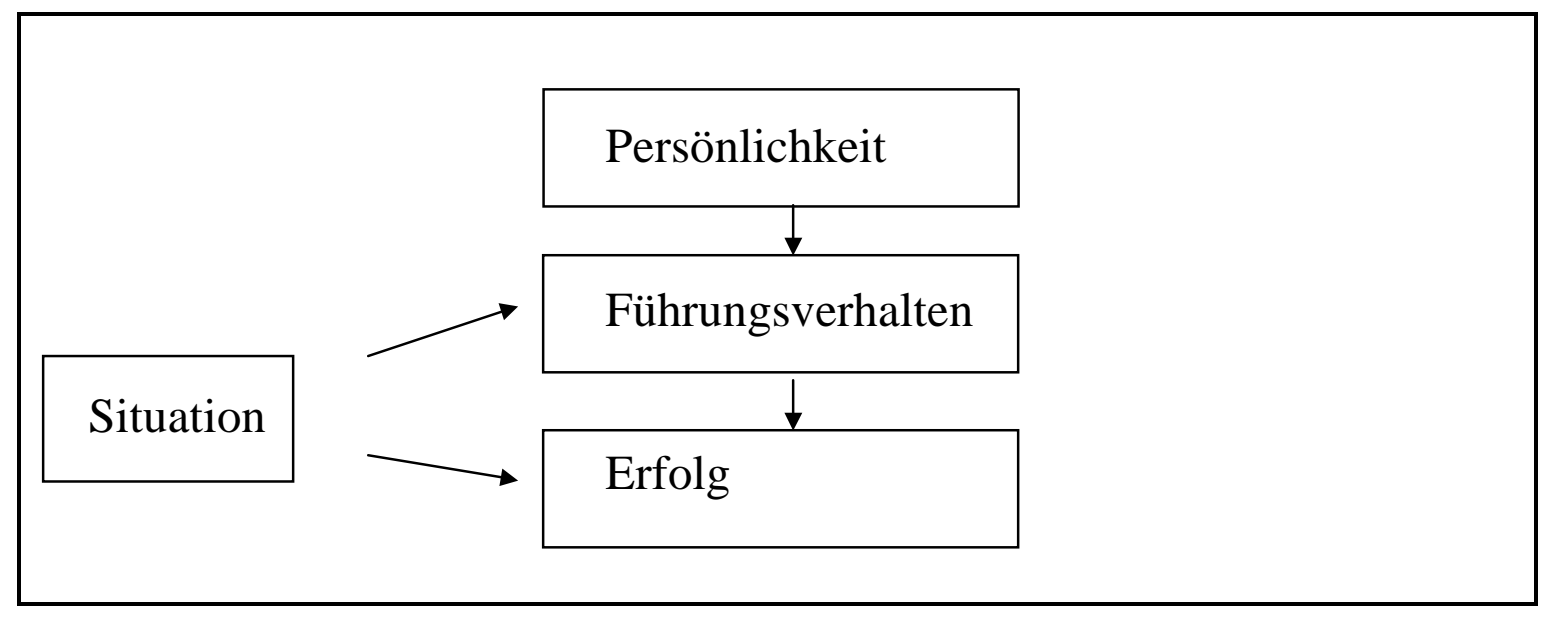

\section{Abbildung 3-1 Bedingtheit des Erfolges durch Person und Situation} (VON ROSENSTIEL, 1993, S. 339)

Das Modell zeigt, dass sowohl Merkmale der Person des Managers als auch Merkmale der Situation den Managementerfolg beeinflussen.

Es berücksichtigt die wichtigsten Kritikpunkte an den verschiedenen, oben beschriebenen, Ansätzen der Vergangenheit (SARGES 1995, S. 7):

„Der Erfolg einer Organisation lässt sich nicht alleine auf der Ebene des individuellen Manager-Verhaltens festmachen.

Verhalten ist weniger aus Persönlichkeitsmerkmalen allein erklärbar, als vielmehr aus den Gegebenheiten der Situation bzw. aus der Interaktion von Person und Situation.

Selbst wenn es persönlichkeitsbedingte Einflüsse eines Managers auf den Erfolg gibt, ist ein in einer bestimmten Position bzw. in einem bestimmten Umfeld erfolgreicher Manager nicht notwendig in jeder anderen Position bzw. in jedem anderen Umfeld erfolgreich.“

Zusätzlich relativiert dieses Modell die oft (NEUBERGER, 1990; STAEHLE, 1992; KOMPA, 1995) geäusserte Kritik der Glorifizierung des Berufsstandes der Manager, in dem der Manager allein für Erfolg verantwortlich ist. 
Einige Kritikpunkte am Modell von von Rosenstiel müssen dennoch angefügt werden.

Ein Einfluss der Person auf den Erfolg kann nur angenommen werden, wenn davon ausgegangen wird, dass die Einflussmerkmale der Persönlichkeit - über die Zeit und auch über verschiedene Situationen hinweg - konsistent oder zumindest hinreichend stabil sind. Und genau diese Annahme ist nicht eben unbestritten und hat die Persönlichkeits-Psychologie der letzten Jahre in der sogenannten Person-SituationDebatte (oder auch Interaktionismus-, bzw. Konsistenz-Debatte) massiv beschäftigt (vgl. PERVIN, 1989; MOSER, 1991). Der Ansatz von MISCHEL (1977) hilft in dieser Diskussion vermittelnd: er unterscheidet „starke“ (strukturierte, eingeschränkte Lösungs- und Verhaltensmöglichkeiten) und "schwache“ Situationen mit vielen Verhaltensmöglichkeiten. Darauf aufbauend konnte gezeigt werden, dass Persönlichkeitsmerkmale nur in schwachen Situationen entsprechende Verhaltensprognosen zulassen (ICKES, 1982).

Da Management-Situationen in der Regel schwache Situationen mit mehreren Möglichkeiten sind, kann das Modell von Rosenstiel in der Management-Diagnostik eingesetzt werden. Es wird deshalb dieser vorliegenden Untersuchung zu Grunde gelegt.

Zusätzlich zum Modell von von Rosentiel wird in dieser Arbeit davon ausgegangen, dass die einzelnen Bausteine in einer gegenseitigen Wechselwirkung stehen und nicht nur einseitig in eine Richtung wirken. Als zusätzliche Hypothese wird auch ein Zusammenhang zwischen Situation und Persönlichkeit postuliert.

In einer ersten Fassung wurde ein zusätzlicher Baustein „Sportverständnis“ als fünfter Baustein in das Modell integriert. Nach der Durchführung des empirischen Pre-Tests wurde aber dann wieder darauf verzichtet.

Dies ergibt folgendes Arbeitsmodell:

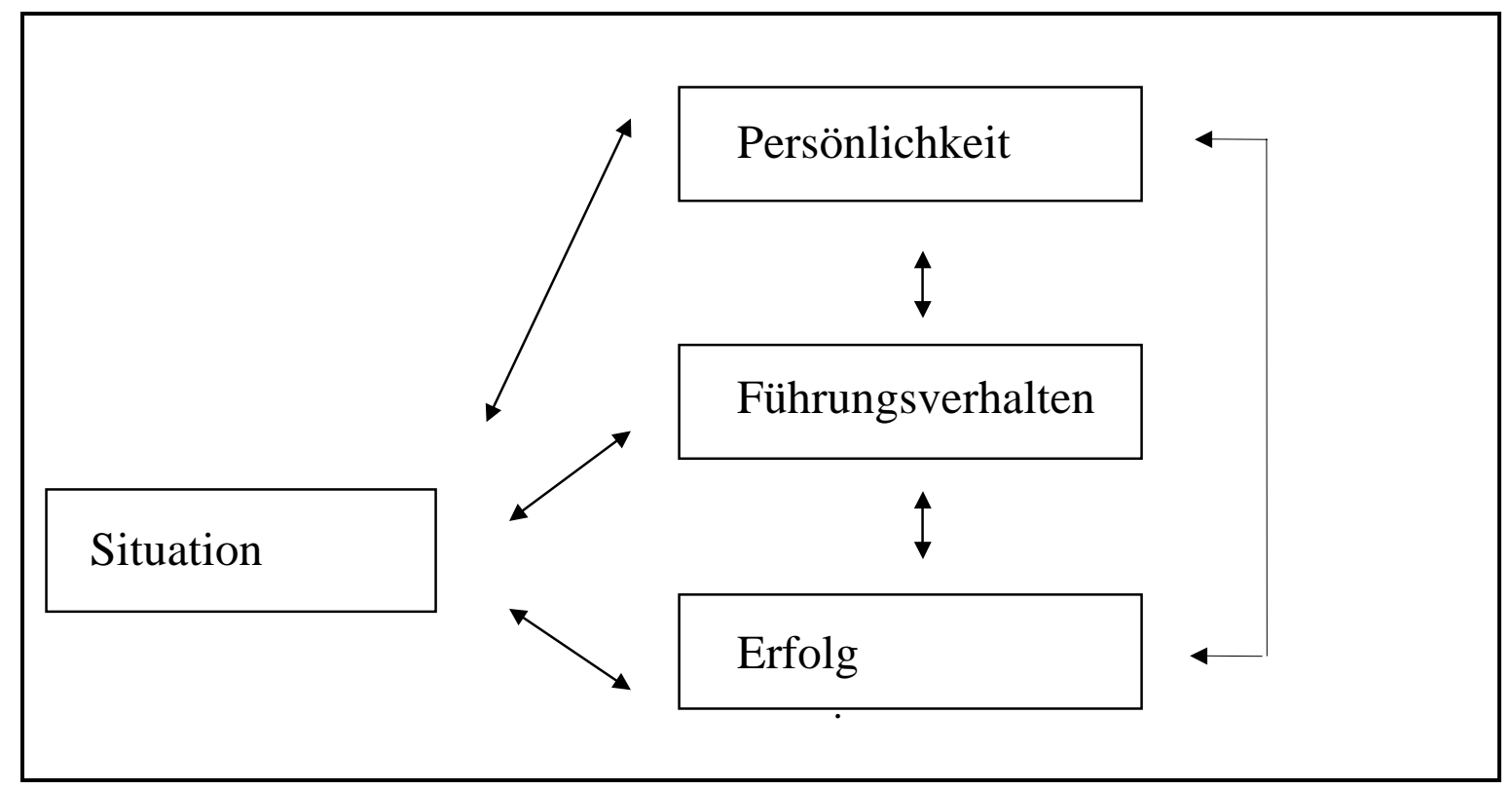

Abbildung 3-2 Arbeitsmodell der vorliegenden Arbeit 
(nach SCHMID 1998)

\subsection{Trainerpersönlichkeit und Erfolg}

Im Zusammenhang mit der Untersuchung über Erfolg und Persönlichkeit (vergleiche Kapitel 3.1 Persönlichkeit und Erfolg Seite 14 wurden auch spezifisch die Zusammenhänge von Trainerpersönlichkeit und Erfolg betrachtet. Die Werke von HENDRY (1972 und 1974) waren dabei grundlegend. Weitere Untersuchungen stammen von BIRD (1977), VOS STRACHE (1979) und CHELLADURAI \& CARRON (1981). Insgesamt waren aber die Resultate eher ernüchternd, wie auch HORN (1992, pp. 183) feststellte:

"Although research conducted to test these theories (Zusammenhänge Persönlichkeit und Erfolg) in educational and industrial settings resulted in some support for this approach to leadership effectiveness, efforts to apply these same theories to the sport context resulted in minimal succes."

Angespornt durch diese Situation entwickelte CHELLADURAI eine sportspezifische Theorie über den Zusammenhang zwischen Persönlichkeit und Erfolg. Wie dies aber bereits in anderen Bereichen, wurde auch hier die Situation als zusätzliche Determinante des Erfolges eingeführt (vergleiche Kapitel 3.4 Person und Situation und Erfolg. Seite 15 .

Das entstandene Modell stellt sich folgendermassen dar:

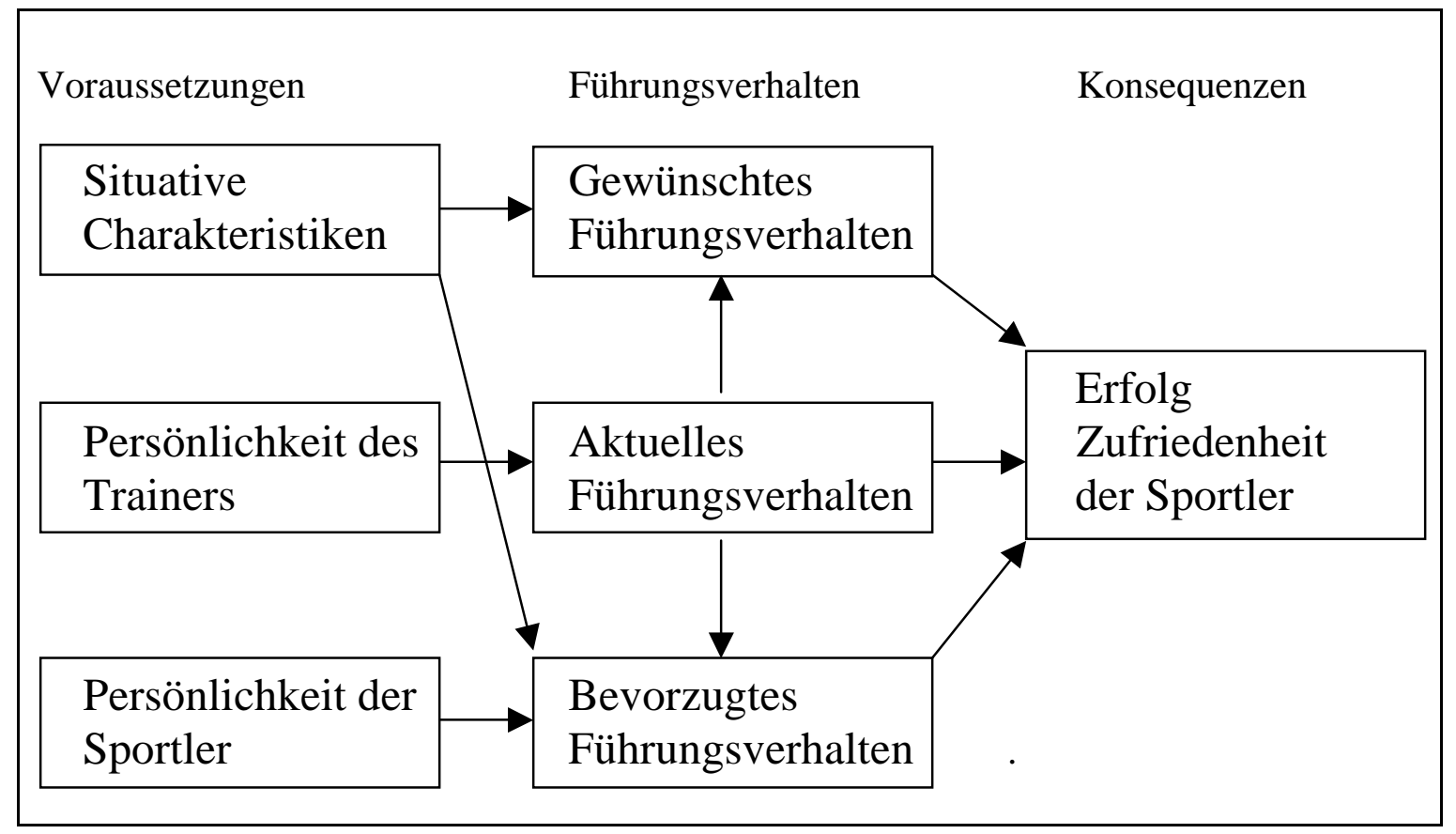

Abbildung 3-3 Multidimensionales Führungsmodell für den Sport

(nach CHELLADURAI 1980) 
Die Parallelen mit dem bereits vorgestellten Modell von VON ROSENSTIEL (1993) sind klar ersichtlich. Die verschiedenen Faktoren des Modells sind Persönlichkeit, Situation, Führungsverhalten und Erfolg. Unterschiede oder Ergänzungen gibt es in folgenden Bereichen:

- Im Bereich Persönlichkeit werden die Persönlichkeiten der Sportler oder der Trainierten ebenfalls noch miteinbezogen.

- Im Bereich des Führungsverhalten wird noch zwischen bevorzugtem Verhalten (Sollverhalten aus der Sicht der Athleten) und nötigem Verhalten (Sollverhalten aus der Sicht der Situation) und aktuellem Ist-Verhalten des Trainers aufgrund seiner Persönlichkeit differenziert.

- Im Bereich des Erfolgs wird zwischen sportlichem Erfolg und Zufriedenheit der Mannschaft oder der Sportler unterschieden.

Als Quintessenz aus seinen Untersuchungen postuliert CHELLADURAI (1980), dass der Erfolg und die Zufriedenheit der Athleten umso grösser sei, je näher die drei Verhaltensdimensionen zusammenrückten.

Überträgt man das System wieder auf die Betriebswirtschaft, so könnte man statt Athleten die Mitarbeiter oder die Kunden einsetzen. Auch dort kann man behaupten, dass der Erfolg umso grösser ist, je näher Kundenerwartungen und deren Erfüllung durch das Management zusammenkommen. Die einzelnen Dimensionen

- nötiges Verhalten durch die Situation bedingt

- gewünschtes Verhalten aus Kundensicht

- aktuelles Verhalten aufgrund der Persönlichkeit des Fitnesscentermanagers

werden denn auch alle im Fragebogen der vorliegenden Untersuchung erhoben. In diesem Sinne ist auch der Autor zusammen mit SAGE (1973) und CHELLADURAI (1985) der Meinung, dass Coaching oder Trainieren ähnlich ist wie Management und deshalb die Persönlichkeits-Erfolgs-Modelle aus der Management-Literatur sich auch auf den Bereich der Trainer anweden lassen und umgekehrt. Gemeinsames Sporttreiben schweisst nicht nur ein Sportteam zusammen, sondern ist als Teil der Corporate Culture auch ein Faktor zur Verhinderung der Personalfluktuation (vergleiche KRÜGER 1981).

Parallelen lassen sich auch im Bereich der Rollenkonflikte zeigen, denen sowohl Manager als auch Trainer in ihrer Funktion ausgesetzt sind. Dabei gibt es viele verschiedene Theorien, welche Rollen der Manager einnehmen muss. Neben den Unterschieden in den eigentlichen Rollendefinitionen ist allen Theorien gemeinsam, dass sich gewisse Rollen widersprechen, beziehungsweise fast nicht miteinander vereinbar sind. Diese Spannung muss eine Führungskraft aushalten können.

Ganz ähnlich ist auch der Trainer mit verschiedenen, teils widersprüchlichen Rollen konfrontiert, die er erfüllen sollte.GABLER \& HAHN (1974) fanden bei einer Untersuchung bei 68 Bundestrainern aus Deutschland und 34 Nationaltrainern aus der Schweiz die folgenden 6 Rollen:

- Der Trainer als Fachmann

- Der Trainer als Motivationsvermittler

- Der Trainer als Motivationsbekräftiger
- Der Trainer als Lehrer

- Der Trainer als Betreuer

- Der Trainer als Funktionär 
HAGEDORN (1995) formuliert in seinem Artikel „Trainer: die vereinigten Widersprüche der Persönlichkeit" auf sehr pointierte Art und Weise die folgende Rollen:

- Herr der Sportler, Knecht des Managements, Sündenbock, Minipapst, Ersatz-Vater, Über-Ich

- Initiator, Arrrangeur, Regisseur, Sündenbock und Delinquent

- Jäger und Gejagter

Trotz der übertriebenen Formulierungen klingen gewisse Wahrheiten an. Es gibt auch klare Entsprechungen zu den Rollen von GABLER \& HAHN: Fachmann entspricht dem Minipapst, Lehrer dem Ersatz-Vater oder Arrangeur, Motivator dem Jäger, Funktionär dem Knecht des Managements, etc.

Einzelne dieser erwähnten Rollen sind je nach Autor wichtiger als andere für den Erfolg oder werden besonders erwähnt:

- Die Rolle des Trainers als Experte und Fachmann: u.a. BROMME 1992, WOERMANN \& HANKE 1994, FRANKE 1995

- Die Rolle des Trainers als Pädagoge und Erzieher: u.a. CACHAY \& GAHAI 1989, KRÜGER 1989, KRÜGER 1991, WON, SCHARENBERG \& KRÜGER 1996

- Die Rolle des Trainers gegenüber der Gesellschaft: KRÜGER 1995, CACHAY 1995

Zusammenfassend kann zur Trainerpersönlichkeit folgendes gesagt werden:

- Den erfolgreichen Trainer schlechthin gibt es nicht. Erfolg ist immer abhängig von der Situation, den beteiligten Sportlern, den Zielsetzungen und eben der Persönlichkeit des Trainers.

- Wichtiger als das perfekte Spielen einer Rolle ist der Umgang mit den verschiedenen, teils widersprüchlichen und unvereinbaren Rollen des Trainers.

- Je nach Persönlichkeit fallen einem die einen oder anderen Rollen einfacher. Entsprechend sollte man sich als Trainer das jeweilige Umfeld aussuchen, in dem diejenigen Rollen, die man ohne grossen Aufwand gut übernehmen kann, die erfolgswirksamsten im jeweiligen Bereich sind.

Dies deckt sich wieder mit der Meinung vieler Management-Trainer, dass es den erfolgreichen Manager an sich gar nicht gibt. Es gibt Hinweise, dass gewisse Persönlichkeiten in gewissen Situationen und Umfeldern erfolgreicher sind als andere (vergleiche Kapitel 3.1 Persönlichkeit und Erfolg, Seite 14). Erfolgsvoraussagen aufgrund der Persönlichkeit können deshalb nur sehr spezifisch für eine bestimmte Situation abgegeben werden.

In diesem Sinne sind allgemeine Aussagen über den erfolgreichen Trainer in der vorliegenden Arbeit eben nur in allgemeiner Form als Referenz zu gebrauchen. Wie bereits oben erwähnt, kann aufgezeigt werden, dass die Anforderungen an einen erfolgreichen Trainer oder Coach sehr ähnlich sind wie die Anforderungen an einen erfolgreichen Manager. 
Im Sinne einer Vereinfachung und für den Zweck des Vergleichs von Sport und Management werden in dieser Arbeit Trainer und Coach gleichgesetzt. Dies in Übereinstimmung mit FIEDLER in SCHNABEL (1993, S. 78), der Coach wie folgt definiert:

„Trainer, Ausbilder eines Sportlers oder einer Mannschaft.

Der Begriffsinhalt hat sich mit der Leistungssportentwicklung gewandelt. Aus dem ehemaligen Berater und Zurufer (z.B. in englischen Ruderwettkämpfen) wurde besonders in den Sportspielen der verantwortliche Trainer der Mannschaft. Dabei wird in verschiedenen Ländern zwischen den Funktionen eines Trainers (konditionelle Ausbildung; Einüben von Standards usw.) und denen eines Coach (Strategie der Mannschaftsführung; Mannschaftsaufstellung; Taktikkonzeption; Führung der Spieler und der Mannschaft im Wettkampf usw.) unterschieden.“

Eine detaillierte Differenzierung der Begriffe siehe HOTZ (1993, zitiert in HOTZ 1997).

Die untenstehenden Beispiele von HOTZ (1997) und KRAUS \& WESTERMANN (1995) zeigen die Parallelen von Trainer/Coach und Manager deutlich. Die einzelnen Punkte sind vom Autor numeriert, um die ähnlichen Aussagen zu kennzeichnen.

Der gute Coach

- Ist glaubwürdig (1) und überzeugend (2), vorbildlich (3) und vertrauensstiftend (4)

- Ist psychologisch (5) und sportlich kompetent (6)

- Hat eine auch ethisch geleitete Verantwortung (7)

- Kann das Sportler-Verhalten modifizieren (8) und und deren Reserven mobilisieren (9)

- Rückt die eigene Person in den Hintergrund (10)

- Erkennt, was Wettkämpfer brauchen und handelt in deren Sinne (11)

- Kann sich in andere hineindenken (12)

- Kann vermitteln (13)

- Denkt positiv (14), strahlt Selbstvertrauen aus (15)

\section{Tabelle 3-1 Der gute Coach}

(HOTZ, 1997, S. 136)

Der gute Manager

- Besitzt Glaubwürdigkeit (1)

- Besitzt Kritikfähigkeit (5) und die Fähigkeit, sich mitzuteilen (2)

- Ist einfühlsam (12), tolerant und vertrauenerweckend (4)

- Ist Diagnostiker und Stratege (8)

- hat eine hohe Sach- und Sozialkompetenz (6 und 5)

- Ist Ausbildner und Vorbild (3)

- Orientiert sich an den Bedürfnissen des Kunden (11)

- Ist Seelsorger und Puffer $(12,13)$

- ist Moderator und Vermittler (13)

- ist Teamentwickler

- Besitzt emotionale Stabilität (14) und eine grosse Eigenmotivation 


\section{Tabelle 3-2 Der gute Manager}

(KRAUS \& WESTERMANN, 1995, S. 115)

Der gute Manager, wie auch der gute Trainer, muss sich nach zwei Kriterien orientieren: Effizienz und Effektivität.

\section{Effizienz}

Effizienz bezieht sich auf das Verhältnis von Input und Output. Die Frage heisst: „Mit welchem Aufwand wurde das Ziel erreicht?"“.

\section{Effektivität}

Effektivität bezieht sich auf das Erreichen des Ziels. Die Frage lautet: „Wurde das Ziel erreicht?“.

Effektives Handeln ist auch ohne Effizienz vorstellbar: Das Ziel wurde zwar erreicht, der Aufwand war jedoch gewaltig. Das Spiel wurde zwar gewonnen, die Spieler mussten jedoch so ans Limit gehen, dass sie sich auf die nächste Spiele im Turnier, die unmittelbar folgen, kaum wieder erholen werden.

Auf der anderen Seite ist auch Effizienz ist ohne Effektivität möglich: Das Oberziel, schwarze Zahlen zu schreiben wurde zwar nicht erreicht, der Output konnte aber bei gleichbleibendem Input massiv gesteigert werden.

Optimal ist natürlich eine Kombination von effektivem und effizientem Handeln.

In der amerikanischen Literatur wird „efficiency“ definiert als „,doing things right“, „effectiveness“ als „,doing the right things“ (HODGE \& ANTHONY, 1988, S. 300). 


\section{Die Situation}

\subsection{Die Fitnesslandschaft Schweiz}

Die Schweizerinnen und Schweizer sind Weltspitze, wenn es um die Mitgliedschaft in einem Fitnesscenter geht. Gemäss einer Untersuchung von MARTI (1997) haben 13\% aller Schweizer 1997 ein Fitnessabonnement gelöst.

Damit ist in der Schweiz eine Sättigung erreicht. Die Fitnessbranche befindet sich auf dem Produktelebenszyklus im Stadium der Reife. Die Anzeichen eines gesättigten Marktes sind denn auch in der Fitnessbranche nicht zu übersehen:

- Wenig Wachstum

- meistens qualitativ hochstehende Angebote

- Verbesserung werden vor allem im qualitativen Bereich realisiert

- Anstrengungen zur Ausdehnung des Kundenkreises finden weniger im Kerngeschäft als v.a. im Bereich der komplementären Angebote statt

In der Schweiz sind im Bereich der komplementären Angebote hauptsächlich zwei Stossrichtungen festellbar: einerseits vermehrte Betonung des Präventionscharakters von Fitnesstraining (vgl. auch Kapitel 4.3, Qualitop. Seite 26) und Ausdehnung des Trainings in Richtung Rehabilitation und Medizinische Trainingstherapie (MTT). Diese Trends konnten von SCHMID (1999) im Gebiet mit der grössten Fitnesscenterdichte der Schweiz, im Zürcher Oberland und im Limmattal, in einer Untersuchung erhärtet werden und decken sich mit den Aussagen von URSPRUNG (1998).

\subsection{Der typische Fitnesscenterbesucher}

Eine Untersuchung Schweizerischen Beratungsstelle für Unfallverhütung bei 15'000 Fitnesscenterbesuchern (von denen 6680 antworteten) ergaben 1998 folgendes Bild des Fitnesscenterbesuchers (BFS, 1998):

Altersstruktur

\begin{tabular}{|l|l|l|}
\hline Alter & Frauen (in \%) & Männer (in \%) \\
\hline$<25$ & 11 & 10 \\
\hline $25-34$ & 37 & 31 \\
\hline $35-44$ & 25 & 25 \\
\hline $45-54$ & 17 & 19 \\
\hline $55-64$ & 7 & 9 \\
\hline$>65$ & 3 & 6 \\
\hline
\end{tabular}

Tabelle 4-1 Altersstruktur der Fitnesscenterbesucher

Alle Altersgruppen sind vertreten. Man kann eine Verschiebung des Besucher-Alters nach oben feststellen. Mehr als die Hälfte der Besucherinnen sind älter als 35, bei den Männern beträgt der Anteil sogar fast 60\%. Die über 55 Jährigen machen bei den Frauen 
bereits $10 \%$ aller Kunden aus, bei den Männern sogar 15\%. Diese Zahlen widerspiegeln einerseits die generelle Überalterung in der Bevölkerung, zeigen aber auch, dass Fitnesstraining vom Image ,jung, schön, heisser Dress“ weggekommen und auch für ältere Menschen salonfähig geworden ist.

Ausbildung
\begin{tabular}{|l|l|}
\hline Ausbildung & Anteil in \% \\
\hline Grundschule & 5 \\
\hline Berufslehre & 72 \\
\hline Studium & 23 \\
\hline
\end{tabular}

Tabelle 4-2 Ausbildungsstand der Fitnesscenter-Besucher

Man kann eine Übervertretung von Studiumsabgängern im Vergleich zum gesamtschweizerischen Durchschnitt (BFS 1998) feststellen. Einerseits mag dies daran liegen, dass Studierende an den Hochschulen oft ein sehr gutes (und billiges!) Fitnessangebot haben, dies stark nützen und dann später nicht auf diese gute Gewohnheit verzichten wollen.

Andererseits zeigt dies einmal mehr die bekannte Tatsache, dass gebildetere Schichten mehr Sport treiben und mehr für ihre Gesundheit tun, als ungebildetere (MARTI, MAEDER \& CALMONTE, 1999).

Hauptaktivität im Center

\begin{tabular}{|l|l|l|}
\hline Tätigkeit & Frauen (in \%) & Männer (in \%) \\
\hline Kraft an Geräten & 13.2 & 24.6 \\
\hline Ausdauer an Geräten & 6.0 & 7.2 \\
\hline Kraft und Ausdauer & 47.8 & 61.6 \\
\hline Aerobic, Gymnastik & 30.4 & 3.4 \\
\hline Sauna, Solarium & 1.2 & 1.0 \\
\hline Anderes & 1.4 & 1.4 \\
\hline
\end{tabular}

Tabelle 4-3 Hauptaktivität der Fitnesscenter-Kunden

Die Hauptaktivität ist kombiniertes Kraft- und Ausdauertraining, sowohl bei den Frauen, als auch bei den Männern. Das reine Kraftbolzen steht bei einem Viertel der Männer immer noch im Vordergrund. Dies ist aber deutlich weniger als noch wenigen Jahren. Hier zeigen sich erste Wirkungen der grossen Anstrengungen der Center, das Herzkreislauftraining $\mathrm{zu}$ propagieren und $\mathrm{zu}$ einem fast obligatorischen Teil des Fitnesstrainings zu machen - unter anderem auch auf Druck von QUALITOP.

Andererseits ist auch Krafttraining für Frauen zum Thema geworden. Fast die Hälfte aller Frauen betreibt neben Ausdauertraining auch noch spezifisches Krafttraining an den Geräten. 
Grundsätzlich kann man festhalten, dass sich beide Geschlechter in ihrem Fitnessverhalten immer mehr annähern. Je mehr das Fitnesstraining die Gesundheit im Vordergrund steht (die Hauptmotivation für beide Geschlechter), desto kleiner werden auch die Unterschiede bei den einzelnen Trainingsinhalten.

Trainingshäufigkeit

\begin{tabular}{|l|l|}
\hline Häufigkeit pro Woche & Anteil in \% \\
\hline$<1 \mathrm{x}$ & 2.3 \\
\hline $1 \mathrm{x}$ & 23.2 \\
\hline $2 \mathrm{x}$ & 51.7 \\
\hline$>2 \mathrm{x}$ & 22.8 \\
\hline
\end{tabular}

Tabelle 4-4 Trainingshäufigkeit der Fitnesscenter-Besucher

Drei Viertel aller Bescher kommt zwei oder mehr Male zum Fitnesstraining. Dies ist eine erfreulich hohe Zahl an regelmässigen Besuchern. Einerseits zeigt hier die Aufklärungsarbeit der Trainingsleiter Wirkung (,,1 x pro Woche reicht knapp für die Fitnesserhaltung, Fortschritte sind aber damit nicht zu erreichen"), andererseits will der Kunde das doch recht teure Abonnement auch ausnützen.

Trainingsmotivation

\begin{tabular}{|l|l|l|}
\hline Hauptmotivation & Frauen (in \%) & Männer (in \%) \\
\hline Gesundheit & 56.2 & 51.0 \\
\hline Figurerhaltung & 14.2 & 12.2 \\
\hline Ergänzungstraining & 5.4 & 15.0 \\
\hline Rehabilitation & 18.4 & 17.6 \\
\hline Aerobic als Sport & 4.4 & 0.1 \\
\hline Bodybuilding als Sport & 0.1 & 1.8 \\
\hline Anderes & 1.3 & 2.3 \\
\hline
\end{tabular}

Tabelle 4-5 Motivation für den Fitnesscenter-Besuch

Die Hauptmotivation ist mit grossem Abstand die Gesundheit. Es zeigt sich damit eine Verlagerung weg vom klassischen Körper- (und damit Muskel)-training im Sinne der Stilisierung des perfekten Körpers hin zu einem umfassenderen Fitness-Training im Sinne einer ganzheitlichen Gesundheit und als Primärprävention. Zusätzlich motivieren die immer häufigeren Zivilisationskrankheiten im Sinne einer Sekundärprävention zu Fitnesstraining.

Ausführliche Gedanken zu dieser Neuausrichtung des Fitnessverständnisses finden sich im Kapitel 21.3 Erkenntnisse über die Wachstumsfaktoren der Branche Seite 158. 


\subsection{Qualitop}

Im Folgenden wird die Organisation QUALITOP vorgestellt. Die Informationen dafür stammen aus QUALITOP 1999, EIGENMANN 1997 und 1998 sowie aus MARTIN \& MARTI 1998.

Anfangs der 90er Jahre führten nach und nach einzelne Krankenversicherer für entsprechend Zusatzversicherte Kunden und Kundinnen einen dazumal noch Fitnessbonus genannten Präventionsbeitrag ein. Mit diesem Beitrag sollten einerseits fitnesstreibende Versicherte für ihr gesundheitsförderndes Verhalten belohnt werden und andererseits wurde für Noch-nicht-Trainierende ein Anreiz geschaffen, aktiv etwas zur Erhaltung bzw. Steigerung der Gesundheit zu tun.

Mit der Einführung des Präventionsbeitrags trafen die Krankenversicherer ein Bedürfnis vieler Kunden, das sich darin widerspiegelte, dass innert kurzer Zeit zahlreiche Versicherte von diesem Angebot Gebrauch machten.

Dies führte zu steigenden Kosten der Krankenkassen und zur Diskussion, ob die Ausschüttung eines Präventionsbeitrags nach dem Giesskannenprizip überhaupt sinnvoll sei. Wer die Gesundheit von Versicherten durch Fitnesstraining verantwortungsvoll und gezielt fördern will, muss eine Garantie dafür haben, dass das finanziell unterstützte Training auch tatsächlich nachhaltig positiv wirkt. Es musste ein Instrument geschaffen werden, das erlaubte, die Qualität des Angebotes in Fitnesscentern zu beurteilen.

In dieser Absicht schlossen sich die vier grössten Krankenversicherer der Schweiz (Helsana, Konkordia, SWICA und Wincare) in der Interessengemeinschaft QUALITOP zusammen. QUALITOP ist eine Non-Profit-Organisation und rechtlich gesehen ein Verein mit den vier Krankenversicherern als Mitglieder. Die Leitung der laufenden Geschäfte liegt in der Hand eines Geschäftsführers und des kleinen Teams der Gechäftsstelle in Winterthur. Ein Fachbeirat mit Vertretern aus der Fitnessbranche, der Eidgenössischen Sportschule in Magglingen und dem Präventionsbereich steht dem Vorstand bzw. dem Geschäftsführer beratend zur Seite. Somit fliesst auch neues Knowhow sofort in die QUALITOP-Tätigkeiten ein.

QUALITOP erarbeitete einen Qualitätskriterien-Katalog, anhand dessen eine Überprüfung der Qualität in den Fitnesscentern möglich wurde. Damit wurde eine Abgrenzung von präventiv wirkendem, gesundheitsförderndem Fitnesstraining gegenüber konsumorientierter, fitnessartiger Freizeitbeschäftigung angestrebt. Nur Kunden, die in QUALITOP-zertifizierten Centern trainieren, erhalten auf Antrag den Präventionsbeitrag zugesprochen.

Die Überprüfung der Center wird durch ein Team von über 40 Testern gewährleistet, die speziell für diese Tätigkeit ausgebildet wurden.

„Aus dem ehemaligen Marketinginstrument der Krankenkassen „Fitnessbonus“ wurde ein Instrument der Attraktivitätssteigerung der zertifizierten Center und ein klarer Wettbewerbsvorteil gegenüber den Nicht-Zertifizierten.“ (EIGENMANN 1998, S. 12) 
So ist es denn auch nicht erstaunlich, dass momentan 292 Center der etwa 400 Fitnessinstitutionen und -Centern in der Schweiz das QUALITOP-Zeritifkat besitzen.

Die qualitativ guten QUALITOP-Center schienen für diese vorliegende Untersuchung eine sehr gute Untersuchungsgruppe darzustellen. Dazu kommt, dass durch das Interesse der Organisation QUALITOP an den Ergebnissen die Adressen der zertifizierten Center zur Verfügung gestellt wurden.

\subsection{Situative Variablen der vorliegenden Untersuchung}

Situative Variablen wie zum Beispiel Verkehrslage, Konkurrenzsituation oder angebotene Infrastruktur können entscheidendere Faktoren für den Erfolg eines Fitnesscenters sein als die anderen Bausteine des Untersuchungsmodells wie Persönlichkeit, Führungsverhalten oder Sportverständnis.

Deshalb ist es sehr wichtig, diese situativen Variablen genau zu erfassen.

Von folgenden situativen Faktoren ist vorstellbar, dass sie einen Einfluss auf den Erfolg eines Fitnesscenters haben:

Situative Erfolgsfaktoren

- Konkurrenz-Situation

- direkte Konkurrenten mit gleichem Angebot

- allgemeine Fitnesscenter-Dichte der Region

- Substitutions-Produkte wie Fitnessfarm, Wellnessferien oder Polysportanlagen

- Mitarbeiter-Situation

- Allrounder oder Spezialisten

- Ausbildungsstand, Kompetenz

- Freundlichkeit

- Verhältnis Festangestellte zu Teilzeitangestellte

- Chef auf Platz

- Wirtschaftliche und gesellschaftliche Situation

- Konjunktur, Rezession, Inflation, Kreditvergabe-Politik der Banken

- Fitness- und Gesundheitstrends, Körperkult(ur), Schönheitsideale

- Verkehrslage

- zentral gelegen, an der Peripherie

- Erreichbarkeit

- mit Auto: genügend Parkplätze vorhanden

- mit öffentlichen Verkehrsmitteln: Verbindungen, nahe Haltestellen 
- Infrastruktur

- grosszügige Platzverhältnisse

- modern und hell eingerichtet

- neue Maschinen

- allgemeine Sauberkeit

- Palette des Angebots

- Arzt oder Physiotherapeut im selben Gebäudekomplex

- Abonnements-Möglichkeiten

- Jahresabo

- 10er-Abo

- Zonen-Abos

- Zeiten-Abos)

- Kundenservice

- Kinderbetreuung

- Prbetrainings

- Trainingsberatung

- Abholservice vom Bus

- Ambiente

- Events

- Soziale Anstrengungen des Centers

- grosser Bar-/Treffpunkt-Bereich

- Mitgliederausflüge vom Center aus organisiert, Wettbewerbe

- Vermittlung von Trainingspartnern

\section{Tabelle 4-6 Situative Variablen}

Bei dieser Fülle wird schnell klar, dass im begrenzten Rahmen dieser Untersuchung nicht alle möglichen Einflussfaktoren berücksichtigt werden können.

Im Sinne einer Voruntersuchung werden deshalb diese vielen Variablen auf ein paar wenige relevante Faktoren reduziert (vergleiche 8.4 Baustein Situation, Seite 49. 


\section{Die Persönlichkeit}

\subsection{Exkurs zur Persönlichkeitspsychologie}

Die Persönlichkeitspsychologie befasst sich mit der Frage, inwieweit sich die Persönlichkeit als Erklärung und zur Vorhersage von individuellen Differenzen im Verhalten von Menschen beiziehen lässt. Dabei werden drei Richtungen unterschieden.

\subsubsection{Personale Ansätze}

Modelle dieser Richtung sehen die Determinanten von Verhalten innerhalb der menschlichen Person und postulieren, dass Verhalten aufgrund personinterner Eigenschaften zustande kommt. Die bereits zitierte „great-man-theory“ (vergleiche 3.1. Persönlichkeit und Erfolg Seite ) von CARLYLE (1841) oder GALTON (1869) mit Bestimmungsgrössen wie „Charisma“ oder „Ausstrahlung“ wäre ein typischer Vertreter dieses Ansatzes.

\subsubsection{Situative Ansätze}

Anhänger dieser Richtung unterstellen, das menschliches Verhalten den Prägekräften der jeweiligen Situation unterliegt. Die sogenannte „Nachfragetheorie“ von HOFSTÄTTER (1966) zum Beispiel besagt, dass es von der Situation der Gruppe, dem Führungsbedarf und den Erwartungen der Geführten abhängt, wer zum Führer aufsteigt.

\subsubsection{Interaktionistische Ansätze}

Sie stellen die vermittelnde Position zwischen den personalen und situativen Ansätzen dar und gehen davon aus, dass menschliches Verhalten weder durch die Person noch die Situation allein hinreichend erklärt werden kann, sondern dass beide Faktoren einen Einfluss haben. Die „Kontingenztheorie“ von FIEDLER (1967) war eines der ersten Modelle des interaktionistischen Ansatzes.

In der modernen Managementliteratur dominiert der integrative Ansatz des Interaktionismus.

„Im Managementbereich hat der Interaktionismus mit dem Konzept der Situationsselektion die Sichtweise auf generelle und spezifische Prädiktoren der Managementeignung eröffnet. Demnach sind Situations- und Personmerkmale nur durch wechselseitige Bezugnahme beschreibbar." (DIETERICH \& SOWARKA, 1994).

Deshalb wurde dieser Arbeit das Führungsmodell VON ROSENTIEL, 1993 (vergleiche Abbildung 3-1, Bedingtheit des Erfolges durch Person und Situation zugrunde gelegt, das eine Weiterentwicklung des „Kontingenz“-Ansatzes von FIEDLER darstellt und in diesem Sinne dem Interaktionismus zuzuordnen ist. 


\subsection{Eigenschaftsinventare}

In der Personalbeurteilung und Eignungsdiagnostik wird vorwiegend mit eigenschaftstheoretischen Konzepten gearbeitet. Diese gehen davon aus, dass es im Menschen dauerhafte Dispositionen gibt, die sein Verhalten bestimmen und seine Persönlichkeit ausmachen.

„In wissenschaftlicher Hinsicht ist dieser Aussagekern nicht unproblematisch. Dennoch ist das Konzept für Managementzwecke praktisch. Es erlaubt einerseits die Verwendung objektiver Persönlichkeitstests, andererseits die Standardisierung des Beurteilungswesens anhand vorfabrizierter, auf spezifische Erfordernisse des Betriebes zugeschnittene Eigenschaftsinventare." (DIETERICH \& SOWARKA, 1994).

Eigenschaftsinventare versuchen, die riesige Fülle von Eigenschaften (ALLPORT \& ODBERT, 1936, zählten über 18'000 Eigenschaftswörter im englischen Wortschatz) auf eine überblickbare und vergleichbare Zahl zu reduzieren, um Willkür und Subjektivität bei der Anwendung auszuschliessen.

Eines der häufigsten Eigenschaftsinventare ist die „Adjective Check List“ von GOUGH (1952). In der Bibliographie von GOUGH \& HEILBRUNN (1980) werden über 700 Artikel zu diesem Beurteilungsverfahren aufgelistet.

\subsection{Typologien}

Die konsequenteste Form der Reduzierung stellen sogenannte Typologien dar, die versuchen, die Vielfalt des menschlichen Wesens auf eine geringe Zahl von Typen zu reduzieren. Einige der klassischen Typologien (z.B. von KRETSCHMER, SHELDON, JUNG oder SPRANGER) wurden durch amerikanische Management-Forscher für eignungsdiagnostische Zwecke weiterentwickelt.

\subsubsection{Strong Interest Inventory}

Das Strong Interest Inventory von STRONG (1943) baut auf den sechs idealen Typen von SPRANGER (1925) auf und gruppiert die Berufs- und Karriereinteressen der Probanden nach „General Occupational Themes“: Realistic, Investigative, Artistic, Social, Enterprising und Conventional. Es wurde millionenfach benutzt und in der Berufs- und Karrierenberatung eingesetzt.

Für die Management-Diagnostik wurden die sechs Occupational Themes durch MCALLISTER (1990) erweitert durch die Begriffe aufgabenorientiert-pragmatisch (R), datenorientiert-theoretisch (I), unabhängig-kreativ (A), menschenorientiert-humanistisch $(\mathrm{S})$, machtorientiert-kompetitiv (E) und produktorientiert-effizient $(\mathrm{C})$. 


\subsubsection{Myers-Briggs Type Indicator MBTI}

Dieser Test von BRIGGS \& MYERS (1977) geht auf die psychologische Typenlehre von JUNG (1921) zurück und unterscheidet vier Präferenzen zwischen

- Extraversion und Introversion: Einstellung zur Umwelt, aussen- (E) oder innen- (I) orientiert.

- Sensing und Intuition: Wahrnehmung, sinnlich (S) oder intuitiv (N)

- Thinking und Feeling: Beurteilung des Wahrgenommenen, analytisch (T) oder gefühlsmässig $(\mathrm{F})$.

- Judging und Perceiving: Lebenseinstellung, beurteilend (J) mit Bezug auf $(\mathrm{T}) /(\mathrm{F})$ oder wahrnehmend $(\mathrm{P})$ in Bezug auf $(\mathrm{S}) /(\mathrm{N})$.

Aufgrund der acht einzelnen Ausprägungen werden vier Präferenzen berechnet, die dann einen Typ (z.B. INTP) von möglichen 16 Typen darstellen.

Zum MBTI liegen eine Fülle von Forschungsarbeiten in Kombination mit anderen Persönlichkeits- Leistungs- und Beobachtungsskalen vor (CARLSON 1985, MURRAY 1990, MYERS \& MCCAULLEY 1985, u.a.)

„Es verwundert daher nicht, dass der MBTI über das Anwendungsgebiet der Bildungs- und Berufsberatung hinaus auch ein bevorzugtes Instrument der Karriereberatung abgibt. Aufgrund der hieraus resultierenden Datenfülle zu verschiedenen Managerstichproben sind der organisationspsychologische Einsatz des MBTI weit verbreitet (zu den Vor- und Nachteilen des MBTI als Instrument der Persönlichkeitsmessung vgl. BELL, 1993).“ (DIETERICH \& SOWARKA, 1994).

\subsubsection{Weitere Management-spezifische Typologien}

Über den MBTI hinaus gibt es Typologie-Konzepte, die speziell auf die Bedürfnisse der Management-Diagnostik hin entwickelt wurden. So z.B. die Zweitypen-Konzepte „Sachorientierung“ und „Menschenorientierung“ von BLAKE \& MOUTON (1986), „A“ und „B“ von FRIEDMANN \& ROSENMANN (1974) oder „X“ und „Y“ von MCGREGOR (1970). Dreitypen-Konzepte bestehen von MARCH \& SIMON (1976) und BASS \& AVOLIO (1990), Viertypenkonzepte schliesslich von SCHEIN (1980) oder MEYER \& DAVIS (1992).

Durch die starke Reduktion auf zwei bis vier Typen leidet jedoch die Aussagekraft der Resultate.

\subsection{Auswahl des Erhebungsinstruments}

Im Sinne des Arbeitsmodells der vorliegenden Arbeit, welches Situation und Person miteinbezieht, kommt nur ein interaktionistischer Ansatz in Frage.

Bei der Entscheidung, ob ein Eigenschaftsinventar oder eine Typologie verwendet werden soll, waren folgende Faktoren massgebend: 


\section{Validität, Reliabilitat, Objektivität}

Sowohl Eigenschaftsinventare wie auch Persönlichkeitstypologien sind häufig verwendete Tests, die in aufwändigen Verfahren standardisiert und validiert wurden. Diese Kriterien sind darum kein Argument, den einen oder anderen Test zu bevorzugen.

\section{Korrelationsmöglichkeiten}

Die Resultate von Korrelationen einzelner Typen, die eine Kombination aus verschiedenen Eigenschaften darstellen, sind wenig aussagekräftig, da nicht abgeschätzt werden kann, wie gross der Einfluss der einzelnen Eigenschaften auf den eigentlichen Typen ist. In diesem Sinne sind Eigenschaftsinventare besser geeignet, zu einzelnen Eigenschaften konkrete Aussagen zu machen.

Unterteilt man jedoch die Typen in die jeweiligen Eigenschaften, so können wieder valable Aussagen gemacht werden. Die MBTI-Typen beispielsweis können auf die vier Eigenschaftspaare E-I, N-S, T-F und J-P reduziert werden und sind damit einfacher auswertbar als eine Adjektivliste mit 15 - 20 Begriffen.

\section{Auswertung}

Bei den Persönlichkeitstypologien bestehen oft bereits vorgefertiget Auswertungen und Beschreibungen $\mathrm{zu}$ den einzelnen Typen. Gerade der MBTI-Test ist sehr gut dokumentiert und erlaubt es, den Probanden quasi als Belohnung für ihre Beteiligung an der Umfrage ein umfassendes Persönlichkeitsprofil zuzustellen. Dass die Sprache der Persönlichkeitsprofile sehr gut allgemein verständlich ist, erhöht natürlich den Wert für den Einsatz bei Laien.

\section{Vergleichsdaten}

Sowohl bei den Eigenschaftsinventaren als auch bei den Persönlichkeitstypen gibt es viele Untersuchungen, die als Vergleichsdaten herbeigezogen werden können. Beim MBTI-Test ist zusätzlich zu erwähnen, dass er gerade in der Management-Literatur öfters zitiert wird und sich deshalb für unsere Fragestellung sehr gut eignet.

Aufgrund dieser Überlegungen wird entschieden, in dieser Arbeit der MBTI-Test zur Ermittlung der Managerpersönlichkeit zu verwenden.

Für Korrelationsanalysen werden die jeweiligen Merkmalsausprägungen der vier Präferenzen verwendet.

Die einzelnen MBTI-Typen werden im Folgenden kurz vorgestellt. 


\subsection{Die verschiedenen Typen des MBTI}

Aus den vier Hauptausprägungen E-I, S-F, T-N und J-P werden 8 Persönlichkeitstypen abgeleitet, die von STÖGER (1996) wie folgt charakterisiert werden:

Der Extrovertierte (E)

- Ist ein guter Redner

- Liebt die Geselligkeit

- Geht von sich aus auf andere zu

- Will beständig die Welt verändern

- Verfügt über grosse Interessensbreite

- Tankt Energie im Kontakt mit anderen

- Beobachtet engagiert das äussere Geschehen
Der Introvertierte (I)

- Ist ein guter Zuhörer

- Schätzt das Alleinsein

- Wartet, dass man auf ihn zugeht

- Reflektiert die Welt

- Verfügt über grosse Interessenstiefe

- Schöpft Energie aus sich heraus

- Beobachtet eher das innere Geschehen

Abbildung 5-1 Die beiden Typen der Ausprägung E - I

Der Intuitive (N)

- Erfasst die Welt intuitiv

- Hat sehr viel Phantasie

- Richtet den Blick in die Zukunft

- Ist kreativ und theoriefreudig

- Verlässt sich auf seinen sechsten Sinn

- Hält den Überblick für das Wesentliche

- Orientiert sich an gedachten Möglichkeiten
Der Sensitive (S)

- Bewegt sich auf dem Boden der Realität

- Ist sehr vernünftig

- Lebt in der Gegenwart

- Vertraut auf Erfahrung

- Verlässt sich auf seine fünf Sinne

- Liebt Details

- Orientiert sich an Tatsachen

\section{Abbildung 5-2 Die beiden Typen der Ausprägung N - S}

Der Denker (T)

- Ist objektiv

- Folgt der Logik

- Entscheidet vom Kopf her

- Erscheint sachlich und kühl

- Geht analytisch an Probleme heran

- Misst sich und andere an Grundsätzen

- Ausgeprägtes Verständnis für Fairness
Der Einfühlsame (F)

- Sieht und lebt die menschliche Seite

- Reagiert persönlich und emotional

- Entscheidet aus Herz und Bauch

- Zeigt und ist empfänglich für Gefühle

- Nähert sich Problemen subjektiv

- Zeigt Anteilnahme

- Pflegt soziale Werte

Abbildung 5-3 Die beiden Typen der Ausprägung T - F 
Der Judger (J)

- Liebt Ordnung

- Plant langfristig

- Hat gern alles geregelt

- Arbeitet ergebnisorientiert

- Trifft Entscheidungen gerne und endgültig
Der Perceiver (P)

- Liebt das kreative Chaos

- Kann sich gut anpassen

- Ist flexibel und spontan

- Ist verfahrens- und prozessorientiert

- Schiebt Entscheidungen gerne auf

Abbildung 5-4 Die beiden Typen der Ausprägung J - P

Zur Vereinfachung reduziert man die 16 Typen auch noch auf sogenannte Kerntypen. Damit sind die Typen gemeint, die durch die beiden mittleren Buchstaben beschrieben werden: SF, ST, NT, NF. Die beiden mittleren Funktionen beschreiben zwei zentrale Funktionen: wie ein Mensch Informationen aufnimmt und wie er daraufhin Entscheidungen fällt. Die vier Kerntypen werden von STÖGER (1996) wie folgt charakterisiert:

Der SF-Typ: der einfühlsame Praktiker

- Verlässt sich auf seine fünf Sinne und pflegt Beziehungen aktiv.

- Arbeitet gewissenhaft, konzentriert sich auf Tatsachen und Fakten

- Arbeitet lieber im Team und ist sehr hilfsbereit

- Engagiert sich weniger um der Aufgabe als um der Kollegen willen

- Nimmt Kritik persönlich und geht Konflikten aus dem Weg

Der ST-Typ: der prinzipentreue Praktiker

- Verlässt sich auf seine fünf Sinne und hat Grundsätze

- Ist ein Arbeitstier und liebt die Details, termintreu und zuverlässig

- Engagiert sich um der Aufgabe und nicht um der Kollegen willen

- Bringt Kritik direkt an und hat keine Angst vor Konflikten

- Für Prozesse auf der Beziehungsebene manchmal blind

Der NT-Typ: der zielverliebte Visionär

- Nimmt Informationen intuitiv auf und entscheidet vom Kopf her

- Sieht das Ziel und die grossen Linien, guter Komplexitätsbewältiger

- Wirkt nach aussen kühl und distanziert, hasst Routinearbeiten

- Legt sich tendenziell mit Autoritäten an

- Scheut keinen Konflikt, bringt Kritik eher global denn konkret

Der NF-Typ: der hilfsbereite Visionär

- Nimmt seine Umwelt intuitiv wahr und legt Wert auf Beziehungen

- Begeistert sich für neue Aufgaben und reisst andere mit

- Hilfsbereit, lässt sich aber gerne unterbrechen, positive Lebenseinstellung

- Übersieht gerne die Details, ist am ganzen Bild interessiert

Abbildung 5-5 Die vier Kerntypen 
Die eigentliche Typologie von MYERS und BRIGGS unterscheidet jedoch 16 verschiedene Typen. Diese Typen einzeln hier zu charakterisieren, würde den Rahmen sprengen. Im Beltz-Testmanual (Myers-Briggs-Typenindikator, 1995) sind sie sehr gut beschrieben. Es sei deshalb an dieser Stelle auf diese Quelle hingewiesen.

Wie bereits erwähnt, wurden diese Beltz-Typenbeschreibungen denn auch den Fitnesscenter-Managern als persönliche Information zugestellt. 


\section{Das Führungsverhalten}

Grundsätzlich lassen sich zwei Ansätze zur Beschreibung des Führungsverhaltens unterscheiden: der analytisch-funktionsorientierte und der empirischhandlungsorientierte Ansatz.

\subsection{Der analytisch-funktionsorientierte Ansatz}

Der analytisch-funktionsorientierte Ansatz geht auf die Sichtweise des französischen Ingenieurs FAYOL (1916) zurück, der Management als Bündel universell nachweisbarer Funktionen in allen Organisationen definierte: prévoir (planen), organiser (organisieren), commander (befehlen), coordonner (koordinieren) und contrôler (kontrollieren).

Diese Sichtweise wurde ergänzt durch die Management-Forscher GULICK und URWICK (1937), die das Modell POSDCORB begründeten (planning, organizing, staffing, directing, coordinating, reporting, budgeting) und durch den Nobelpreisträger in Wirtschaftswissenschaften, SIMON (1945), der die Funktion „Entscheidung“ einbrachte und ihr eine übergeordnete Bedeutung im Management-Prozess attestierte. In der deutschen Management-Literatur werden die Management-Funktionen noch weiter unterteilt, und zwar in sachbezogene (z.B. Planung, Organisation, Kontrolle) und personenbezogene (Personalführung) Funktionen. Bei dieser dualen Aufteilung darf nicht übersehen werden, dass die Trennung in sach- und personenbezogene Komponenten nur analytischen Charakter haben kann, denn in Realität ist stets eine enge Verwobenheit von Sach- und Personenorienierung festzustellen. (STAEHLE 1991, S. 14)

Diese fehlende Realitätsnähe des analytische-funktionsorientierten Ansatzes und die fehlende empirische Fundierung (planen, organisieren, entscheiden, führen und kontrollieren sind empirisch kaum beobachtbar) führten zu einem neuen Modell: dem empirisch-handlungsorientierten Ansatz.

\subsection{Der empirisch-handlungsorientierte Ansatz}

Die Vertreter dieser Forschungsrichtung erheben die Aktivitäten der Manager vor Ort. Mit der Tagebuchmethode erhoben CARLSON (1951) die Arbeitsinhalte von neun schwedischen Top Managern und STEWART (1967) die Arbeitsweise von 160 Top und Middle Managers in britischen Unternehmungen. Der wichtigste Forschungsbeitrag stammt von MINTZBERG (1973), der fünf Top Manager von fünf amerikanischen Unternehmungen während 25 Tagen intensiv beobachtete.

Zusammenfassend lassen sich die Ergebnisse dieser Forschungsrichtung folgendermassen charakterisieren (nach NEUBERGER 1990, S. 158f.):

- Die Manageraktivitäten sind kurz, abwechslungsreich und stark fragmentiert - es wechseln wichtige und unwichtige, dringliche und nicht dringliche, bedeutsame und unbedeutsame Inhalte.

- Der Manager reagiert mehr als er agiert. 
- Etwa die Hälfte der Aktivitäten dauert weniger als neun Minuten; nur 10\% dauern länger als eine Stunde.

- Bei den meisten Aktivitäten herrscht in der Regel enormer Zeitdruck.

- Der Manager betreibt in Wirklichkeit sehr viel stärker kommunikative, interpersonale Aktivitäten als es in den Lehrbüchern der Betriebswirtschaftslehre gemeinhin dargestellt wird.

- Er verbringt oft mehr als 50\% seiner Zeit mit Nicht-Unterstellten, wie seinen eigenen Vorgesetzten, Kollegen in der Firma oder Kollegen anderer Firmen, mit Mitarbeitern anderer Funktionsbereiche oder Betriebe oder mit Aussenstehenden. Dies weist auf die Bedeutsamkeit und Komplexität seines Netzwerkes hin.

- Bei den oben beschriebenen Tätigkeiten hat er nicht nur die Führungsrolle inne, sondern noch eine Vielzahl anderer Rollen.

Zwei Erkenntnisse aus der obenstehenden Liste müssen besonders hervorgehoben werden.

„Der Manager betreibt in Wirklichkeit sehr viel stärker kommunikative, interpersonale Aktivitäten.“

Dies ist eine klare Kritik am analytisch-funktionalen Ansatz und ein deutlicher Beleg für die Wichtigkeit der verhaltenswissenschaftlichen Management-Forschung.

„Er hat nicht nur die Führungsrolle inne, sondern noch eine Vielzahl anderer Rollen.“

Diese Erkenntnis wird auch von STAEHLE (1991) gestützt.

Ein zentrales Problem dieser Berufsgruppe (gemeint sind die Manager) besteht ganz offensichtlich darin, sich auf rasch wechselnde Situationen und damit auf neue Rollenanforderungen einstellen zu können, dabei aber ihre Identität und unverwechselbaren Persönlichkeitsmerkmale nicht zu verlieren. (STAEHLE 1991, S. 15)

Als Management-bedeutsame Rollen wurden anzahlmässig zwischen 10 (MINTZBERG, 1973) und 24 (STAEHLE, 1991) identifiziert. Grundsätzlich lassen sich alle sogenannten Rollenkonzepte auf den von MINTZBERG (1973) verfassten Vorschlag zurückführen. Er wird deshalb an dieser Stelle vorgestellt:

Die 10 Führungsrollen

Interpersonelle Rollen

Repräsentant

Führer

Koordinator
Informationsbezogene Rollen Informationssammler Informationsverteiler Informant von externen Gruppen
Entscheidungsrolle

Unternehmer

Krisenmanager

Ressourcenzuteiler

Verhandlungsführer

\section{Abbildung 6-1 Die 10 Führungsrollen}

(nach MINTZBERG, 1973) 
Das Rollenkonzept hat den Vorteil, dass es erheblich verhaltensnäher ist, als das Funktionenkonzept und deshalb besser geeignet ist für empirische Untersuchungen, die sich mit Verhalten oder Tätigkeiten befassen.

Es ist jedoch anzufügen, dass Rollen keine starren Verhaltensschablonen sind, sondern durch Erwartungen und die jeweilige Situation beeinflusst werden.

Rollen sind nicht fertig, objektiv und generell gültig, sondern einmalig und fragwürdig. Zwar haben alle Handelnden aneinander Erwartungen, die zunächst gesellschaftsüblichen Typisierungen folgen; was jemand aber aus seiner Rolle macht, ist - abgesehen von den je unterschiedlichen Konstellationen - vor allem von ihm und seinen Mitspielern abhängig. (NEUBERGER, 1990, S. 86)

Die bessere Messbarkeit ist jedoch für eine Untersuchung entscheidend, so dass die oben erwähnte Einschränkung in Kauf genommen werden kann. Dazu kommt, dass das Rollenkonzept die als so wichtig befundenen interpersonalen Abläufe (vgl. NEUBERGER, 1990, S. 158 ff und STAEHLE 1991, S. 15) und die Relevanz der Situation (vgl. Abbildung 3-1 Bedingtheit des Erfolges durch Person und Situation mit der erwähnten Unterscheidung von schwachen und starken Situationen (MISCHEL, 1973) besser berücksichtigt als das Funktionenkonzept.

Das Rollenkonzept war denn auch Grundlage für viele weitere Forschungsarbeiten über das Managerverhalten (z.B. BUSSON, LARSON, VICORS \& NESS, 1981; MILLS, ROBEY \& SMITH, 1985, ROACH, 1986; LUEDER, 1986; MARTINKO \& GARDNER, 1990), wobei wiederholt geäussert wurde, dass ein wirklich aussagekräftiges Kategorisierungssystem für Managerverhalten fehle. Zu diesem Zweck haben LUTHANS \& LOCKWOOD (1984) das sogenannte Leader Observation System LOS entwickelt, das als hoch reliables Messinstrument für Managerverhalten dienen soll. Dieses System teilt Managerverhalten in 12 Kategorien ein.

\section{Die 12 LOS-Kategorien}

$\begin{array}{ll}\text { Planning/coordinating } & \text { planen/koordinieren } \\ \text { staffing } & \text { Personal führen } \\ \text { training/developing } & \text { ausbilden } \\ \text { decision making/problem solving } & \text { Probleme lösen/entscheiden } \\ \text { processing paperwork } & \text { Administration/Papierarbeit } \\ \text { exchanging routine information } & \text { Sitzungen/Informationsaustausch intern } \\ \text { monitoring/controlling performance } & \text { überwachen, kontrollieren } \\ \text { motivating/reinforcing } & \text { motivieren, bestärken } \\ \text { disciplineing/punishing } & \text { disziplinieren, bestrafen } \\ \text { interacting with outsiders } & \text { Akquisition, Marketing } \\ \text { managing conflict } & \text { Konfliktmanagement } \\ \text { socialising/politicking } & \text { Repräsentation, soziale Verpflichtungen }\end{array}$

\section{Abbildung 6-2 Die 12 LOS-Kategorien}


Assuming that the LOS accurately classifies the major types of managerial behavior, it appears to be an ideal tool for investigating the relationship between psychological types and observed managerial behavior. (GARDNER \& MARTINKO, 1990, S.35)

\subsection{Auswahl des Erhebungsinstruments}

Im Sinne unseres Arbeitsmodells, das Verhalten als einen Baustein betrachtet, kommt nur ein empirisch-handlungsorientierter Ansatz in Frage.

Die Anwort auf die Frage, welches Rollenkonzept zu verwenden ist, wird durch folgende Faktoren bestimmt:

\section{Validität, Reliabilitat, Objektivität}

Die verschiedenen Führungstätigkeiten müssen den verschiedenen Rollen zugeteilt werden können. Je klarer die Grenzen zwischen den verschiedenen Rollen oder Funktionen sind, desto einfacher ist dies möglich und desto aussagekräftiger werden die Resultate.

Da die Führungstätigkeiten in einzelnen Teilen Berufsgruppen-abhängig sind, ist es wichtig, dass die Liste der Führungstätigkeiten für die spezifische Situation angepasst werden.

In diesem Sinne eignet sich das Leader-Observation-System von LUTHANS UND LOCKWOOD besser als das zwar populärere Rollenkonzept von Mintzberg, da es bereits für die Zusammenhänge von Führungsverhalten und Persönlichkeit angepasst wurde.

Es wird deshalb entschieden, dieses Verfahren in dieser Untersuchuang ebenfalls anzuwenden. Um noch klarere Resultate $\mathrm{zu}$ erhalten, werden die einzelnen Führungstätigkeiten durch den Verfasser noch mit ganz spezifischen Inhalten für den Fitnesscenter-Manager gefüllt. 


\section{Der Führungserfolg}

Bevor auf die Frage des Erfolgs von Fitnesscenter-Managern eingegangen wird, seien ein paar grundsätzliche Überlegungen zum Thema Erfolgsmessung angeführt.

\subsection{Problematik der Erfolgsmessung}

Messen ist die Zuordnung von Zahlenwerten zu Eigenschaften von Objekten, welche nach bestimmten Regeln erfolgt. (BORTZ, 1984 und MARTIN, 1989). Dabei beziehen sich die Zuordnungsregeln immer nur auf gewisse Eigenschaften respektive Attribute der originär zu messenden Objekte (in unserm Fall Managererfolg). Folglich ist Erfolg per se nicht messbar, sondern nur einzelne Merkmale des Erfolgs.

Gerade hier beginnen aber die Schwierigkeiten. Je nachdem aus welcher Warte aus man Erfolg betrachtet, sind ganz andere Kriterien wichtig. Der Inhaber des Centers definiert vielleicht Erfolg vor allem über den erzielten Gewinn oder die Rendite. Für den Geschäftsführer sind zufriedene Kunden und steigende Abonnementszahlen Kriterien für den Erfolg. Der Kunde wiederum hat nochmals ganz andere Vorstellungen.

Anliegen der Erfolgsmessung dieser Arbeit muss darum sein, möglichst viele Aspekte des Erfolgs eines Centers zu berücksichtigen. Als erste Auslegeordnung von möglichen Erfolgskriterien kann das im nächsten Unterkapitel beschriebene Modell dienen.

\subsection{Aufbau eines Messsystems}

Der Aufbau eines Erfolgsmessungssystems kann nach MOCK \& GROOVE (1979) in fünf Schritten erfolgen:

1. Identifikation von Umfeld und Zweck des Messsystems

Dabei sind folgende Massebenen vorstellbar (nach EUSKE, 1984):

- Anstrengung und Einsatz (Input)

- Effektivität (Output)

- Angemessenheit (Output im Verhältnis zu den Bedürfnissen)

- Effizienz (Verhältnis von Input zu Output)

- Prozess (Output als Funktion des Inputs)

\section{Identifikation möglicher Massgrössen}

Es werden diejenigen Massgrössen bestimmt, die am ehesten geeignet sind, die gesuchten Merkmale objektiv zu beschreiben. Objektivität gründet auf folgenden Bedingungen (nach BORTZ, 1984, MOCK, 1976 und EUSKE, 1984):

- Reliabilität (Verlässlichkeit): Mehrere unabhängige Messungen liefern das gleiche Ergebnis.

- Validität (Güte): Die gemessenen Resultate geben die wirklichen Verhältnisse wieder. 
- Meaningfulness (Verständlichkeit): Für den Benutzer verständlich.

\section{Analyse der Eigenschaften der möglichen Massgrössen}

Die gefundenen Massgrössen werden auf ihre Eigenschaften hin geprüft (z.B. Reliabilität oder Validität) und es wird entschieden, ob die Massgrössen für die Messzwecke einsetzbar ist.

\section{Entwicklung und Analyse von neuen Massgrössen}

Oft reichen die bestehenden Massgrössen nicht aus. Es müssen neue Grössen gesucht und auch wieder evaluiert werden.

\section{5. Überprüfung des Gesamtsystems auf Nützlichkeit und Kosten}

Am Schluss müssen die Einzelkomponenten im Verbund überprüft werden und die Kosten in Relation zum erwarteten Nutzen gesetzt werden.

\subsection{Auswahl der Erhebungskriterien}

$\mathrm{Da}$ in diesem Bereich im Unterschied $\mathrm{zu}$ den Bausteinen Persönlichkeit und Führungsverhalten keine standardisierten Tests vorliegen, muss ein eigenes Erhebungsinstrument durch den Verfasser geschaffen werden.

Es wird dabei versucht, möglichst alle fünf von MOCK \& GROOVE (1979) angesprochenen Bereiche zu berücksichtigen.

- Anstrengungen

- Output

- Effektivität

- Effizienz

- Prozess

Die einzelnen Fitnesscenter-spezifischen möglichen Erfolgskriterien der fünf Bereiche werden im Kapitel Grundlagen der Empirischen Untersuchung, Unterkapitel 8.3 (Baustein Erfolg ab Seite 47 ausführlich vorgestellt. Dort wird auch begründet, wieso einzelne Kriterien nicht erhoben werden, obwohl sie sehr aussagekräftig für den Erfolg eines Fitnesscenter-Managers wären.

Die gleichen Anmerkungen können auch zum Baustein „Situation“ gemacht werden. Auch dort sind keine standardisierten Messinstrumente vorhanden. Die Analyse, Diskussion und Entscheidung für die in dieser Arbeit verwendeten Erhebungskriterien für die Situation finden sich im Kapitel Grundlagen der Empirischen Untersuchung, Unterkapitel 8.4 Baustein Situation, ab Seite 49. 


\section{Empirischer Teil}

\section{Grundlagen der empirischen Untersuchung}

\subsection{Baustein Persönlichkeit}

\section{Identifikation der Persönlichkeit}

Für den Baustein Persönlichkeit kann der MBTI-Test unverändert übernommen werden. Für die individuellen Rückmeldungen an die an der Untersuchung teilnehmenden Fitnesscenter-Manager werden die 16 Typen des Originaltests übernommen. Für die Korrelationen mit den anderen Bausteinen werden die vier Präferenzausprägungen verwendet.

\section{Überprüfung des Gesamtsystems}

Die Überprüfung des Gesamtsystems wird durch einen Pre-Test des Gesamtfragepackages bei 5 Fitnesscentern gewährleistet.

Auswertung des Pre-Tests:

Der Fragebogen ist verständlich und kann so übernommen werden.

\subsection{Baustein Führungsverhalten}

Für den Baustein „Führungsverhalten“ können die 12 LOS-Kategorien verwendet werden. Sie werden durch spezifische Beispiele aus der Tätigkeit eines Fitnesscenterleiters konkretisiert.

Als Ergänzung und Erweiterung der LOS-Methode wird nicht nur die zeitliche Belastung der einzelnen der einzelnen Führungsrollen erfragt, sondern ebenfalls erhoben, welche Prioritäten die Manager den einzelnen Tätigkeiten zuordnen.

Einerseits kann so eine branchenspezifische Prioritätenliste der Führungsrollen erstellt werden, andererseits kann auch festgestellt werden, inwiefern Priorität und dafür verwendete Zeit übereinstimmen. Dies ist eine wertvolle Aussage in Hinblick auf die Optimierung der persönlichen Arbeitseffektivität des einzelnen Managers.

\subsubsection{Konkretisierungskriterien der 10 Führungsrollen im Fitnesscenter}

Die folgenden Kriterien wurden nach einem Beobachtungsbesuch in drei Fitnesscentern erarbeitet. 
Planen/Koordinieren

- Wochenplanung, Monatsplanung, Jahresplanung

- Angebotsplanung, Planen von neuen Aktivitäten

- Marketingplanung

- Finanzplanung

Personal führen

- Personal einstellen, Personalgespräche

- Leitbild oder Vision weitergeben

- Coaching

Ausbilden

- Persönliche Weiterbildung

- Ausbildung der MitarbeiterInnen

- Kurse erteilen an externen Schulen

Entscheiden/Probleme lösen

- Krisensitzungen

- Entscheid für neue Anschaffungen, neues Leitbild, Personalentscheide

Administration/Papier- und Computerarbeit

- Finanzen, Buchhaltung

- Korrespondenz

- Rechtliche Abklärungen

- Abo-Management

- Kundenbetreuung

Sitzungen/Informationsaustausch intern

- Teamsitzungen

- internes Kommunikationssystem

Überwachen, kontrollieren

- Rundgänge im Center: Mitarbeiter, Geräte, Sauberkeit

- Kontrolle der Abschlüsse, Kennzahlen, Verkaufszahlen

- Rapporte der Mitarbeiter

Motivieren, bestärken

- Mitarbeitergespräche

- Teamanlässe, Teambildung

- Kundenbetreuung 
Disziplinieren, bestrafen

- Krisengespräche mit ungenügenden Mitarbeitern

- Massnahmen gegenüber lästigen oder nicht zahlenden Kunden

Akquisition, Marketing

- Kundentage, Probetrainings

- PR-Aktionen, Werbung, Messeauftritte

- CI, Atmosphäre

- Kundenbindungsmassnahmen

Konfliktmanagement

- Teamkonflikte

- Konflikte mit Kunden

- Konflikte mit Konkurrenten oder Behörden

Repräsentation, soziale Verpflichtungen

- öffentliche Auftritte, Teilnahme an Veranstaltungen

- Mitarbeit in Gesundheits-Projektgruppen

- Teilnahme an Kontaktapéros

\section{Abbildung 8-1 Kriterien der 10 Führungsrollen eines Fitnesscenter-Managers}

\subsubsection{Vernehmlassung durch Branchenexperten}

In der Vernehmlassung bei drei Fitnesscenter-Leitern wurden die folgenden Ergänzungen oder Änderungen eingebracht:

Planen/Koordinieren

- neu: Personaleinteilung

Personal führen

- neu: Weiterbildung der Mitarbeiter sicherstellen

- neu: Teambildung

Ausbilden

- keine Ergänzungen

Entscheiden/Probleme lösen

- Reaktion bei Reklamationen von Kunden

Administration/Papier- und Computerarbeit

- keine Ergänzungen 
Sitzungen/Informationsaustausch intern

- keine Ergänzungen

Überwachen, kontrollieren

- keine Ergänzungen

Motivieren, bestärken

- Incentives: Erfolgsbeteiligung, zusätzliche Freitage

- Verantwortung übergeben

Disziplinieren, bestrafen

- keine Ergänzungen

Akquisition, Marketing

- Kontakt zu Banken, Sponsoren oder Gönnern

- Kundengespräche, Probetrainings

Konfliktmanagement

- Keine Ergänzungen

Repräsentation, soziale Verpflichtungen

- Keine Ergänzungen

\subsubsection{Liste der bereinigten Rollenkriterien}

Diese Ergänzungen ergeben folgende bereinigte Kriterienliste:

Planen/Koordinieren

- Wochenplanung, Monatsplanung, Jahresplanung

- Personalplanung, Angebotsplanung, Planen von neuen Aktivitäten

- Marketingplanung, Finanzplanung

- Planung von Events oder Tagen der offenen Tür

Personal führen

- Personal einstellen, Personal-Gespräche

- Leitbild oder Vision weitergeben

- Coaching

- Weiterbildung der Mitarbeiter sicherstellen

- Teambildung

Ausbilden

- Persönliche Weiterbildung

- Ausbildung der MitarbeiterInnen

- Kurse erteilen an externen Schulen 
Entscheiden/Probleme lösen

- Krisensitzungen

- Entscheid für neue Anschaffungen, neue Projekte, neue Mitarbeiter

- Reaktion bei Reklamationen von Kunden

Administration/Papier- und Computerarbeit

- Finanzen, Buchhaltung

- Korrespondenz

- Rechtliche Abklärungen

- Abo-Management, Kundendateien

Sitzungen/Informationsaustausch intern

- Teamsitzungen

- internes Kommunikationssystem

Überwachen, kontrollieren

- Rundgänge im Center: Mitarbeiter, Geräte, Sauberkeit

- Kontrolle der Abschlüsse, Kennzahlen, Verkaufszahlen

- Rapporte der Mitarbeiter

Akquisition, Marketing

- Kundentage

- PR-Aktionen, Werbung, Messeauftritte

- Kontakt zu Banken, Sponsoren oder Gönnern

- Kundengespräche, Probetrainings

Konfliktmanagement

- Teamkonflikte

- Konflikte mit Kunden

- Konflikte mit Konkurrenten oder Behörden

Repräsentation, soziale Verpflichtungen

- öffentliche Auftritte, Teilnahme an Veranstaltungen

- Mitarbeit in Gesundheits-Projektgruppen

- Teilnahme an Kontaktapéros

\section{Abbildung 8-2 Bereinigte Führungstätigkeiten eines Fitnesscenter-Managers}

\subsubsection{Inhalt des Fragebogenteils „Führungstätigkeiten“}

Der Baustein Führungsverhalten wird aufgrund der oben erwähnten Voruntersuchungen angepasst. Die Schlussversion, wie sie in der Untersuchung verwendet wurde, ist im Anhang nachzulesen. 


\subsection{5 Überprüfung des Gesamtsystems}

Die Überprüfung des Gesamtsystems wird durch einen Pre-Test des Gesamtfragepackages bei 5 Fitnesscentern gewährleistet.

Auswertung des Pre-Tests:

Die genaue prozentuale Aufteilung des eigenen Arbeitsaufwands auf die verschiedenen Tätigkeiten setzt eigentlich das Führen eines exakten Arbeitsprotokolles voraus. Das Führen eines solchen Arbeitsprotokolls würde jedoch die zeitliche Belastung für die befragten Fitnesscentermanager massiv überschreiten. Die Fragestellung wird so belassen, zumal der erfahrene Manager auch ohne Arbeitsprotokoll genügend genau die zeitliche Verteilung seiner Arbeitstätigkeiten nennen kann.

\subsection{Baustein Erfolg}

Die Erarbeitung der Erfolgskriterien eines Fitnesscenters erfolgt nach dem Vorschlag von EUSKE, 1984 (vergleiche 7.2, Aufbau eines Messsystems. Seite 40.

\subsubsection{Identifikation des Umfelds und möglicher Messgrössen}

Gemäss EUSKE (1984) lassen sich die folgenden Kriterien für den Erfolg eines Fitnesscenters aufstellen:

Input

- Fläche des Centers

- Anzahl zur Verfügung stehender Geräte in den verschiedenen Bereichen (Herz/Kreislauf, Kraft, Wellness, Aerobics/Gymnastik)

- Anzahl der Mitarbeiter

- Eingesetztes Kapital

Output

- Anzahl verkaufte Abos

- Anzahl Mitglieder

Output in Verhältnis zu Bedürfnissen

- Auslastung der Geräte

Effizienz

- Gewinn

- Gesamtkapitalrendite 


\section{Prozess}

- Anzahl Kundengespräche oder Probetrainings

- Marketinganstrengungen

OSTRENGA (1990) hebt hervor, dass es wünschenswert wäre, wenn neben quantitativen finanziellen (z.B. Gesamtkapitalrendite, Gewinn) und nicht finanziellen Informationen (Grösse des Centers, Kundenzahl) auch solche über qualitative Aspekte des Erfolgs (z.B. Kundenzufriedenheit, Mitarbeiterzufriedenheit) erfasst würden, um eine umfassende Erfolgsmessung zu gewährleisten.

Es ist jedoch anzufügen, dass im Fall dieser Arbeit mit der schon relativ komplexen Versuchsanlage das Miteinbeziehen von qualitativen Daten den Rahmen sprengen würde. Aus diesem Grund wird die Erfolgsmessung nur auf quantifizierbare Daten abgestützt.

\subsubsection{Analyse der Eigenschaften der möglichen Massgrössen}

Die vorgeschlagenen Grössen werden in Experteninterviews gesichtet, gerafft und ergänzt. Zusätzlich werden auch die Fitnesscenter-Manager gefragt, welche Erfolgskriterien sie für sich intern anwenden. Die Interviews ergaben, dass die aussagekräftigsten Zahlen (Umsätze, Gewinne und Renditen der Teilbereiche und des Centers im gesamten) kaum in einem schriftlichen Fragebogen zu eruieren sind. Die einzelnen Centerleiter werden sich hüten, solche Zahlen, die auch der Konkurrenz etwas nützen könnten, preiszugeben. Es müssen folglich ,unverdächtigere“ Werte erhoben werden, die trotzdem etwas über den Erfolg eines Centers aussagen. Eine Variante ist, mit Referenzwerten zu arbeiten. Die Center müssen dabei nicht die eigentlichen Zahlen preisgeben, sondern nur deren Entwicklung. Eine weitere Schwierigkeit der Zahlen ist, dass jedes Center seine eigene Buchhaltung hat und je nach Abschreibungs- und Buchungspolitik unterschiedliche Massstäbe bei der Erhebung der Zahlen angewendet werden. Dies wiederum spricht für die Verwendung von Referenzwerten.

Die Kriterien zur Bewertung des Prozesses (Anzahl Kundengespräche oder Probetrainings, Marketinganstrengungen) sind ebenfalls problematisch. Nicht, weil die Center-Manager diese Daten nicht preisgeben würden, sondern weil viele Center diese Daten gar nicht erheben.

Übrig bleiben also die folgenden erhebbaren Kriterien für Erfolg:

Input

- Fläche des Centers

- Gesamtfläche

- Einzelflächen der 4 Bereiche: Herz-/Kreislauf, Kraft, Aerobics/Gymnastik, Wellness.

- Anzahl der Mitarbeiter:

- Anzahl Vollzeit- und Teilzeitangestellten

- Gesamtzahl hochgerechnete 100\%-Stellen

Output

- Entwicklung der Abo-Verkäufe/Mitgliederzahlen

- Anzahl Jahresabonnemente 1995, 1996, 1997

- Anzahl Einzeleintritte 1995, 1996, 1997 


\section{Effizienz}

- Gewinnentwicklung

- Gewinne 1996 und 1997 (Referenzwert: Gewinn 1995 = 100\%)

- Entwicklung der Gesamtkapitalrendite

- Rendite 1996 und 1997 (Referenzwert: Rendite 1995 = 100\%)

\section{Abbildung 8-4 Erhebungskriterien für Erfolg}

\subsubsection{Entwicklung und Analyse neuer Massgrössen}

Eine Entwicklung von neuen Massgrössen drängt sich nicht auf.

\subsection{4 Überprüfung des Gesamtsystems}

Die Überprüfung des Gesamtsystems wird durch einen Pre-Test des Gesamtfragepackages bei 5 Fitnesscentern gewährleistet.

Auswertung des Pre-Tests:

Fragen können so übernommen werden.

\subsection{Baustein Situation}

Für den Baustein Situation wird ebenfalls nach der Methode von EUSKE 1984 (vergleiche 7.2 Aufbau eines Messsystems Seite 40, vorgegangen.

\subsubsection{Identifikation möglicher Massgrössen}

Gemäss den Vorbemerkungen in Kapitel 4.4 Situative Variablen der vorliegenden Untersuchung (Seite 27) werden folgende situativen Variablen vorgeschlagen:

- Konkurrenz-Situation

- Mitarbeiter-Situation

- Wirtschaftliche und gesellschaftliche Situation

- Verkehrslage

- Erreichbarkeit

- Infrastruktur

- Personal

- Abonnements-Möglichkeiten

- Kundenservice

- Soziale Anstrengungen des Centers 


\subsubsection{Analyse der Variablen „Konkurrenz-Situation“}

Die Konkurrenzsituation ist sicher als situative Variable sehr entscheidend. Sie wird deshalb hier eingehend betrachtet.

Klassischerweise unterscheidet man im Bereich der Konkurrenz zwischen direkter und indirekter Konkurrenz:

Direkte Konkurrenz: sie bietet das gleiche Produkt an: z.B. andere Fitnesscenter mit gleichem Angebot.

Indirekte Konkurrenz: sie befriedigt das selbe Bedürfnis: z.B. sich wohlfühlen, soziale Kontakte, Sport treiben. (SEILER, 1991, S. 223)

SEILER (1991) unterscheidet zusätzlich:

Anbieter mit Kundenkenntnissen: Diese verkaufen bereits ein anderes Produkt an die Kunden. Sie besitzen daher gute Marktkenntnisse, so dass sie später Produkte herstellen können, welche die gleichen Kunden befriedigen. (entspricht den indirekten Konkurrenten)

Anbieter mit Produktkenntnissen: Diese befriedigen schon die gleichen Kundenbedürfnisse (z.B. in einer anderen Region), waren aber bis heute nicht in unserem Zielsegment tätig. In diese Kategorie gehören auch Anbieter, die über das technologische Rüstzeug verfügen, um entsprechende Produkte herstellen zu können. (direkter Konkurrent)

Anbieter mit liierten Geschäften: Hier denken wir vor allem an Unternehmen, die in vor oder nachgelagerten Geschäften operieren. Dabei sind vor allem solche Anbieter gefährlich, die in Geschäften aktiv sind, welche für den Erfolg des eigenen Produktes wesentlich sind. Dies gilt etwa für einen Rohstoffhersteller bei einer knappen Rohstoffsituation, oder ein Grossabnehmer des eigenen Produktes, der einen Konkurrenten übernehmen könnte. (indirekter Konkurrent)

Auf das Fitnesscenter übertragen sind also folgende Konkurrenten zu beachten:

Direkte Konkurrenten (oder Konkurrenten mit Produktkenntnissen)

- andere Fitnesscenter mit ähnlichem Angebot

Indirekte Konkurrenten (oder Konkurrenten mit Kundenkenntnissen)

Je weiter man die Motive fürs Fitnesstraining fasst, um so umfangreicher wird die Palette an indirekten Konkurrenten.

Für „Sport treiben“: u.a. Sportvereine, Polysportzentren, Squash- oder Badmintonhallen, Kletterzentrum

Für „Sich in seiner Haut wohl fühlen“: u.a. Sauna, Wellnessfarms, Tai-Chi, Chi-gong

Für „Selbstvertrauen erwerben“: u.a. Kampfsportarten, Rhetorikkurse,

Kommunikationstraining

Für „soziale Kontakte knüpfen“: u.a. Vereine, Discos

Für „Selbstverwirklichung“": u.a. Selbsterfahrungskurse, Freizeitkurse

Für „Gesundheit pflegen“: u.a. Stressmanagement-Kurse, wandern, joggen, biken,

Sauna, Solarium, Massage 
Es wird sofort ersichtlich, dass es zu weit führen würde, wenn man im Rahmen der Berücksichtigung der Konkurrenzsituation auch die indirekten Konkurrenten mit einbeziehen würde.

Deshalb werden in dieser Arbeit nur die direkten Konkurrenten berücksichtigt.

Es wäre denkbar, eine objektive Fitnesscenterdichte $\mathrm{zu}$ berechnen, in dem die Fitnesscneter nach Einzugsgebieten sortiert und die entsprechende Dichte berechnet würde. Im Sinne einer Konkurrenzaussage ist diese objektive Zahl jedoch nicht sehr aussagekräftig: Center in einem Gebiet mit grosser Dichte können mit einem Nischenangebot relativ konkurrenzlos sein, umgekehrt können in einem Gebiet mit kleiner Dichte zwei sehr ähnliche Center sehr stark miteinander konkurrieren.

Auf diese Scheingenauigkeit der objektiven Dichtezahlen wird deshalb verzichtet und für diese Arbeit auf die subjektive Empfindung der Konkurrenz-Situation durch die einzelnen Centerleiter zurückgegriffen. Mit der Überzeugung, dass der jeweilige Manager, der seit drei oder mehr Jahren im Geschäft ist, die Konkurrenzsituation besser beurteilen kann, als eine gerechnete Dichtezahl.

\subsection{3 „Mitarbeiter-Situation“ und „Wirtschaftliche und Gesellschaftliche Situation"}

\section{Mitarbeiter-Situation}

Die Mitarbeiter sind zu einem grossen Teil am Erfolg oder Nicht-Erfolg eines Fitnesscenters beteiligt - wie dies auch später durch die Resultate dieser Studie bestätigt wird. Die zusätzliche Befragung des Personals hätte aber den Rahmen dieser Untersuchung gesprengt. Da jedoch die Mitarbeiterauswahl und -weiterbildung in der Kompetenz des Fitnesscenter-Managers liegt, wird vereinfachend angenommen, dass der Erfolgsbeitrag der Mitarbeiter durch den Teammanager bestimmt wird.

Wirtschaftliche und gesellschaftliche Situation

Das allgemeine wirtschaftliche Umfeld (Konjunktur oder Rezession, Sparmassnahmen des Bundes im Gesundheitswesen, Neues Krankenversicherungsgesetz mit neuen Subventionsrichtlinien, etc.) und auch das gesellschaftliche Umfeld (allgemeines Gesundheitsbewusstsein der Bevölkerung, Stellenwert der persönlichen Gesundheit und Fitness) hat einen grossen Einfluss auf den Erfolg eines Fitnesscenters.

Da für das Untersuchungsgebiet Schweiz diese Einflüsse als für alle Fitnesscenter mehr oder weniger gleich anzunehmen sind, werden sie in dieser Untersuchung nicht speziell berücksichtigt.

\subsubsection{Analyse der übrigen Variablen}

Die Wichtigkeit der anderen Variablen „Verkehrslage, Erreichbarkeit, Infrastruktur, Personal, Abonnements-Möglichkeiten, Kundenservice, Soziale Anstrengungen des Centers" wird in einer Voruntersuchung eruiert, um unwichtige Variablen auszuschalten und die Untersuchung wenn möglich zu vereinfachen. 
Dabei wird wie folgt vorgegangen:

- Besuch von drei ausgewählten Fitnesscentern:

- ein allgemein ausgerichtetes Center mit über 1500 Jahreskunden (Dynamic

Neuhausen)

- ein stark auf Krafttraining ausgerichtetes Center (Kieser Schaffhausen)

- ein eher elitäres Center (Airgate, Zürich).

- Kurzbefragung von je 8 Kunden

Diese Umfrage ergab folgende Resultate:

\section{Wichtigkeit der situativen Variablen aus Kundensicht}

$(n=24$, sehr wichtig $=4$ Punkte, wichtig $=3$ Punkte, spielt eine Rolle $=2$ Punkte, unwichtig $=1$ Punkt))

\section{Sehr wichtig}

Sauberkeit der Nasszone

Freundlichkeit des Personals

Kompetenz des Personals

\section{Wichtig}

Anzahl Parkplätze $\quad 75$

Verschiedene Abo-Möglichkeiten $\quad 73$

Helle, hohe Räumlichkeiten $\quad 70$

Breite des Angebots $\quad 68$

Moderne Maschinen und Geräte 66

Spielt eine Rolle

Zentrale Lage $\quad 55$

Kollegen trainieren hier 47

\section{Unwichtig}

Erreichbarkeit mit öff. Verkehrsmitteln 35

Kindbetreuung 31

\section{Abbildung 8-5 Wichtigkeit der situativen Variablen aus Kundensicht}

Sehr wichtig

Die Auswertung zeigt erstaunlich deutlich, dass die persönlichen Faktoren für die Kunden an erster Stelle stehen. Sowohl die Freundlichkeit des Personals als auch deren Kompetenz erhalten Höchstnoten. Einzig „Sauberkeit der Nasszone“ wird als noch wichtiger bewertet. Dies ist jedoch das einzige technische Kriterium, das als „sehr 
wichtig“ eingestuft wird. Alle anderen technischen Kriterien wie Anzahl Parkplätze, Breite des Angebots, Abo-Möglichkeiten und moderne Maschinen sind nur ,wichtig“.

\section{Wichtig}

Wichtigkeit der Abonnements-Möglichkeiten: Die Kunden möchten ihre individuellen Leistungsbezüge auch individuell bezahlen. Wer mehr Leistung bezieht, zahlt gerne etwas mehr, wer weniger Leistung bezieht, will nicht den anderen Kunden den Eintritt verbilligen.

Die Wichtigkeit der Breite des Angebots weist darauf hin, dass es den NurKrafttraining-Kunden oder die Nur-Aerobic-Kundin immer weniger gibt. Man legt Wert auf die Möglichkeit, abwechseln zu können. Auch wenn es nur das Gefühl ist, die Möglichkeit zu haben und von der Möglichkeit eigentlich gar nicht soviel Gebrauch gemacht wird.

Im Bereich der „wichtigen“ Variablen lässt sich bemerken, dass die Rangfolge eher zufällig ist. Die Punkte-Differenzen sind eher klein und somit die einzelnen Faktoren fast alle gleich wichtig.

Unwichtig

Für diejenigen, die auf die öffentlichen Verkehrsmittel angewiesen sind, ist die Erschliessung des Centers mit öffentlichen Verkehrsmitteln natürlich sehr wichtig. Wegen der wenigen öV-Fahrer fällt dies im Gesamtrahmen aber wenig ins Gewicht. Dasselbe lässt sich für die Kinderbertreuung sagen. Wer Kinder ins Training mitnehmen muss, für den ist Kinderbetreuung das wichtigste Kriterium überhaupt. Da dies jedoch nur für wenige Kunden gilt, fällt dies im Gesamtrahmen nicht gross ins Gewicht.

Eine Umfrage bei den drei Geschäftsführern der drei Center ergab ein in etwa ähnliches Bild. Aus ihrer Erfahrung heraus erwarteten auch sie, dass „Sauberkeit der Nasszone“ und „Freundlichkeit“ als sehr wichtig eingestuft würden. In diesem Bereich kommen bei Ungenügen sofort Reklamationen der Kunden, was auf dessen Wichtigkeit für den Kunden hinweist. Hier lässt sich bemerken, dass sich jedermann zutraut, über Sauberkeit oder Freundlichkeit ein Urteil abzugeben. Die Fachkompetenz der Mitarbeiter hingegen oder der Gerätepark ist schwieriger zu bewerten oder wird weniger direkt im Center bemängelt als zum Beispiel im Gespräch mit Freunden.

Die Voruntersuchung der situativen Variablen zeigte jedoch, dass es schwierig ist, einzelne Variablen von vornherein auszuschliessen und sich nur auf zwei oder drei zu beschränken. Es werden deshalb folgende Fragen gewählt: 
Als wie wichtig beurteilen Sie die folgenden Faktoren aus der Sicht Ihrer Kunden

( 1 = unwichtig, 2 = spielt eine Rolle, 3 = wichtig, $4=$ sehr wichtig) ? Bitte in die erste Kolonne eintragen.

Wie gut erfüllt Ihr Center diese Anforderungen aus Ihrer Sicht $(1=$ gar nicht, $2=$ teilweise, 3 = gut, 4 = sehr gut)? Bitte in die zweite Kolonne eintragen.

Faktor

Breite des Angebots

Moderne Maschinen und Geräte

Sauberkeit der Nasszone

Kinderbetreuung

Abo-Möglichkeiten

Lage

Anzahl Parkplätze

Erreichbarkeit mit öffentl. Verkehrsmitteln

Freundlichkeit des Personals

Kompetenz des Personals

Kollegen trainieren hier

Helle, hohe Räumlichkeiten

Wichtigkeit für Kunden Erfüllt durch mein Center

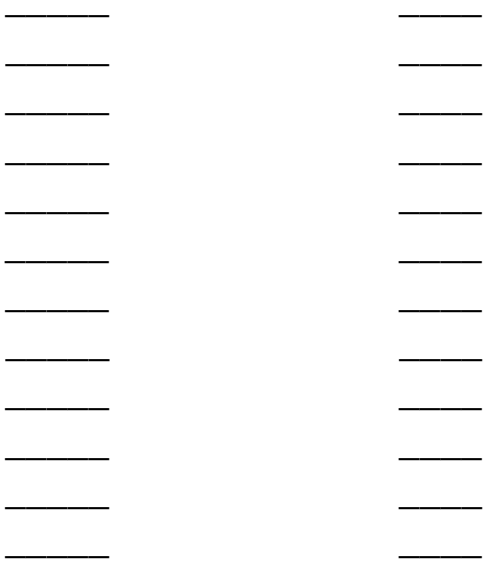

Abbildung 8-6 Erhebung der situativen Variablen

\subsubsection{Entwicklung und Analyse neuer Massgrössen}

Nicht nötig.

\subsection{6 Überprüfung des Gesamtsystems}

Die Überprüfung des Gesamtsystems wird durch einen Pre-Test des Gesamtfragepackages bei 5 Fitnesscentern gewährleistet.

Auswertung des Pre-Tests:

Fragen sind verständlich und klar. Der Fragebogen kann so lanciert werden. 


\section{5 Übersicht über die Untersuchungsanlage}

\subsubsection{Gesamtübersicht}

Zusammenfassend kommen wir zu folgender Untersuchungsanlage:

Untersuchungsgebiet

Erhebungsinstrument

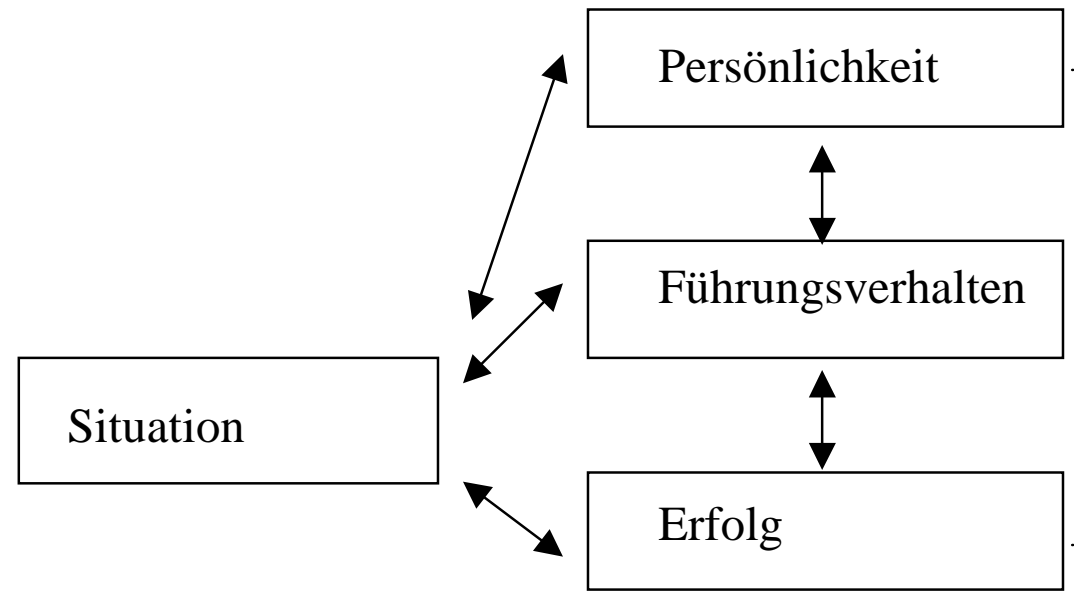

MBTI-Fragebogen

(Original Fragebogen)

LOS von Luthans \& Lockwood (adaptiert für Fitnesscenter)

Sit. Variablen aus Exp.-Interw.

Erfolgskriterien aus ExpertenInterviews und Fragebogen.

\section{Abbildung 8-7 Untersuchungsübersicht}

\subsection{2 Übersicht über die Datenquellen}

Im Folgenden werden die einzelnen Datenquellen kurz charakterisiert.

\section{Baustein Persönlichkeit}

Erhebungsinstument: MBTI-Fragebogen aus der Literatur

Anpassung:

Keine

\section{Baustein Führungsverhalten}

Erhebungsinstument: LOS von LUTHANS \& LOCKWOOD (1984)

Quelle:

Anpassung:

Methodologie:
Aus der Literatur

Mit Konkretisierungskriterien für Fitnesscenter-Manager.

Kriterienerarbeitung durch Autor

Voruntersuchung: Befragung von 3 x 8 Kunden 


\section{Baustein Erfolg}

Erhebungsinstument: Fragebogen

Quelle:

Autor

Anpassung:

Mit Konkretisierungskriterien für Fitnesscenter-Manager.

Methodologie:

Kriterienerarbeitung durch Autor

Vernehmlassung in Experteninterviews: qualitative Interviews mit 3 Fitnesscenterleitern

\section{Baustein Situation}

Erhebungsinstument:

Quelle:

Fragebogen

Anpassung:

Methodologie:

Autor

Vernehmlassung bei Kunden und Managern

Kriterienerarbeitung durch Autor

Voruntersuchung: Befragung von 3 x 8 Kunden

Besprechung der Ergebnisse mit Experten: qualitative Interviews mit 3 Fitnesscenterleitern

\subsection{Auswertungsmöglichkeiten im Überblick}

Die folgende Grafik zeigt die Möglichkeiten der Auswertung in der Übersicht:

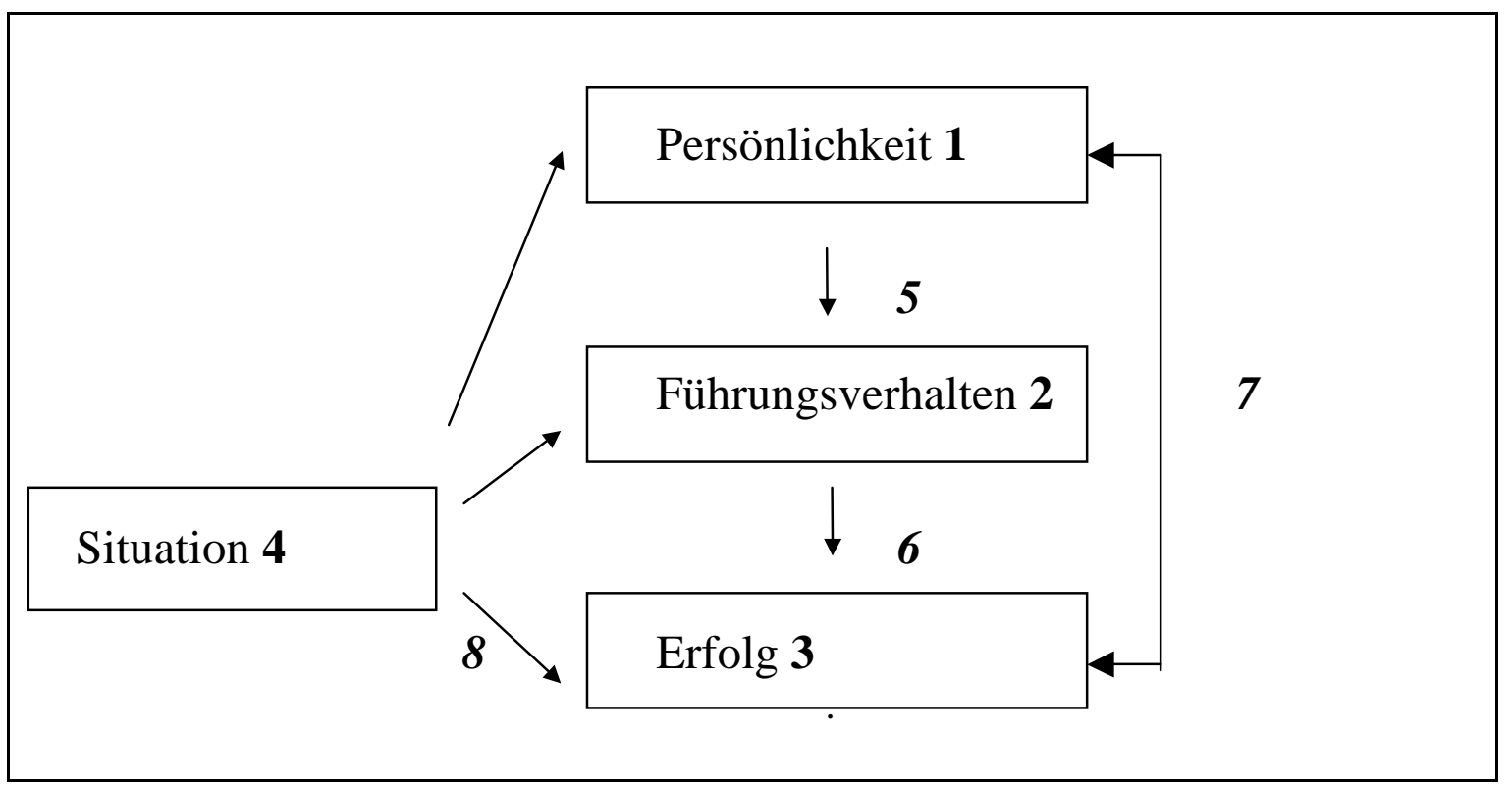

Abbildung 8-8 Auswertungsübersicht 


\subsection{Auswertungen der einzelnen Teilbereiche (Nummern 1 - 4)}

\subsubsection{Persönlichkeit: MBTI (1)}

Persönliches Feedback über Managertyp an alle teilnehmenden Fitnesscenter-Manager

$\rightarrow$ Persönlicher Gewinn für die an der Untersuchung teilnehmenden.

Darstellung der gefundenen Typen

- Verteilung der verschiedenen Typen

- Ist eine Häufung eines Typs feststellbar? Hat dies Gründe?

\subsubsection{Führungsverhalten (2)}

Darstellung der verschiedenen Führungsrollen

- Verteilung der verschiedenen Tätigkeiten

- Welche Führungsrollen brauchen in der Fitnessbranche am meisten Zeit?

\subsubsection{Erfolg (3)}

Darstellung möglicher Erfolgskriterien

- Ergebnisse der Umfrage bei Fitnesscentermanagern

Übersichtsdarstellung

- die erfolgreichen Center - die weniger erfolgreichen Center

8.7.4 Situation (4)

- Übersichtsdarstellung

\subsection{Auswertung der Zusammenhänge (Nummern 5 - 8)}

\subsubsection{Auswirkung der Persönlichkeit auf das Führungsverhalten (5)}

Korrelation der MBTI-Typen mit den Führungsrollen im Fitnessbereich

- Wenden alle Typen für die verschiedenen Führungsrollen etwa gleich viel Zeit auf?

- Bevorzugen einzelne Typen rein zeitlich oder mental einzelne Tätigkeiten?

\subsubsection{Auswirkung des Führungsverhaltens auf den Erfolg (6)}

Korrelation der einzelnen Führungsrollen mit Erfolg

- Welche Führungsrollen sind für den Erfolg in der Fitnesscenterbranche wichtig? Wieso? 


\subsubsection{Auswirkung der Persönlichkeit auf den Erfolg (7)}

- Welche situativen Variablen beeinflussen das Führungsverhalten?

- Welche Variablen beeinflussen den Erfolg?

- Lassen sich lineare Beziehungen herstellen?

\subsubsection{Auswirkung der Situation auf den Erfolg (8)}

- Gibt es Unterschiede zwischen erfolgreichen und nicht erfolgreichen Centern im Bereich der Situation?

- Können kritische Erfolgsfaktoren abgeleitet werden?

- Welche situativen Variablen beeinflussen den Erfolg am meisten? 


\section{Repräsentativität und Validität}

Von den 207 per Anfang Juni 1998 verschickten Fragebogen kamen bis zum 20. Juni 1998 deren 59 (28.6\%) zurück. Mit einem schriftlichen Follow up und persönlichem telefonischen Nachfragen kamen nochmals 23 Fragebogen zusammen. Ein Fragebogen musste für ungültig erklärt werden. Total konnten also 81 der 212 Fragebogen oder $39.3 \%$ ausgewertet werden.

Dieser unüblich hohe Rücklauf für eine schriftliche Befragung mag daher kommen, dass die attraktive Gegenleistung des individuell erstellten Persönlichkeitsprofils den einen oder die andere zum Mitmachen bewogen hat.

Im Folgenden werden verschiedene Parameter der beteiligten Fitnesscenter untersucht, um festzustellen, inwieweit die vorliegenden Ergebnisse als repräsentativ und valid bezeichnet werden können.

\subsection{Geographische Verteilung}

Für die Untersuchung der geographischen Verteilung wird die Schweiz in fünf GrossRegionen mit ihren jeweiligen Kantonen unterteilt:

- Westschweiz (französisch sprechend) WCH:

Genève, Vaud, Fribourg, Jura, Neuchâtel, Valais (französischer Teil)

- Nordwestschweiz NWCH:

Basel Stadt, Basel Land, Solothurn, Aargau

- Zentral- und Südschweiz:

Bern, Wallis (deutscher Teil), Uri, Schwyz, Unterwalden, Ticino, Graubünden

- Zürich ZH:

Zürich

- Ostschweiz OCH:

Schaffhausen, Thurgau, St. Gallen, Thurgau, Appenzell

Die Verteilung der befragten und der antwortenden Center zeigt sich wie folgt:

\begin{tabular}{|l|l|l|l|l|}
\hline Region & \multicolumn{2}{l|}{ Qualitop-Center } & \multicolumn{2}{l|}{ Antwortende Center } \\
\hline & Anzahl & In $\%$ & Anzahl & In \% \\
\hline Westschweiz WCH & 22 & 10.6 & 0 & 0 \\
\hline Nordwestschweiz NWCH & 42 & 20.3 & 18 & 22.2 \\
\hline Zentral- und Südschweiz ZSCH & 43 & 20.8 & 19 & 23.5 \\
\hline Kanton Zürich ZH & 55 & 26.6 & 24 & 29.6 \\
\hline Ostschweiz OCH & 45 & 21.7 & 20 & 24.7 \\
\hline Total & $\mathbf{2 0 7}$ & $\mathbf{1 0 0}$ & $\mathbf{8 1}$ & $\mathbf{1 0 0}$ \\
\hline
\end{tabular}

Tabelle 9-1 Geographische Verteilung der Center 
Diese Verteilung lässt sich folgendermassen darstellen:

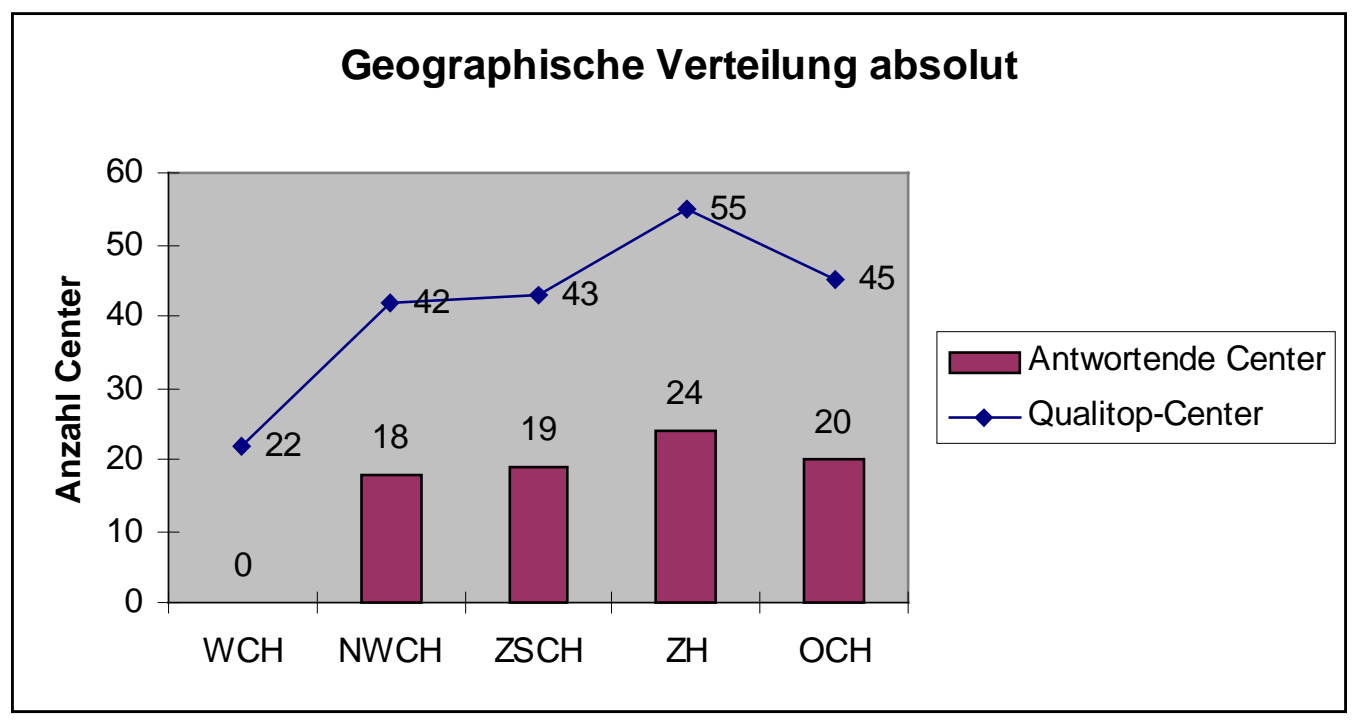

\section{Abbildung 9-1 Geographische Verteilung der Center}

Die beiden Stichproben (befragte Qualitopcenter, $n=207$ und antwortende Center $n=81$ ) sind sich sehr ähnlich - ausser für die Region Westschweiz. Um sie noch besser vergleichen zu können, sind sie auch noch in ihrer prozentualen Verteilung dargestellt.

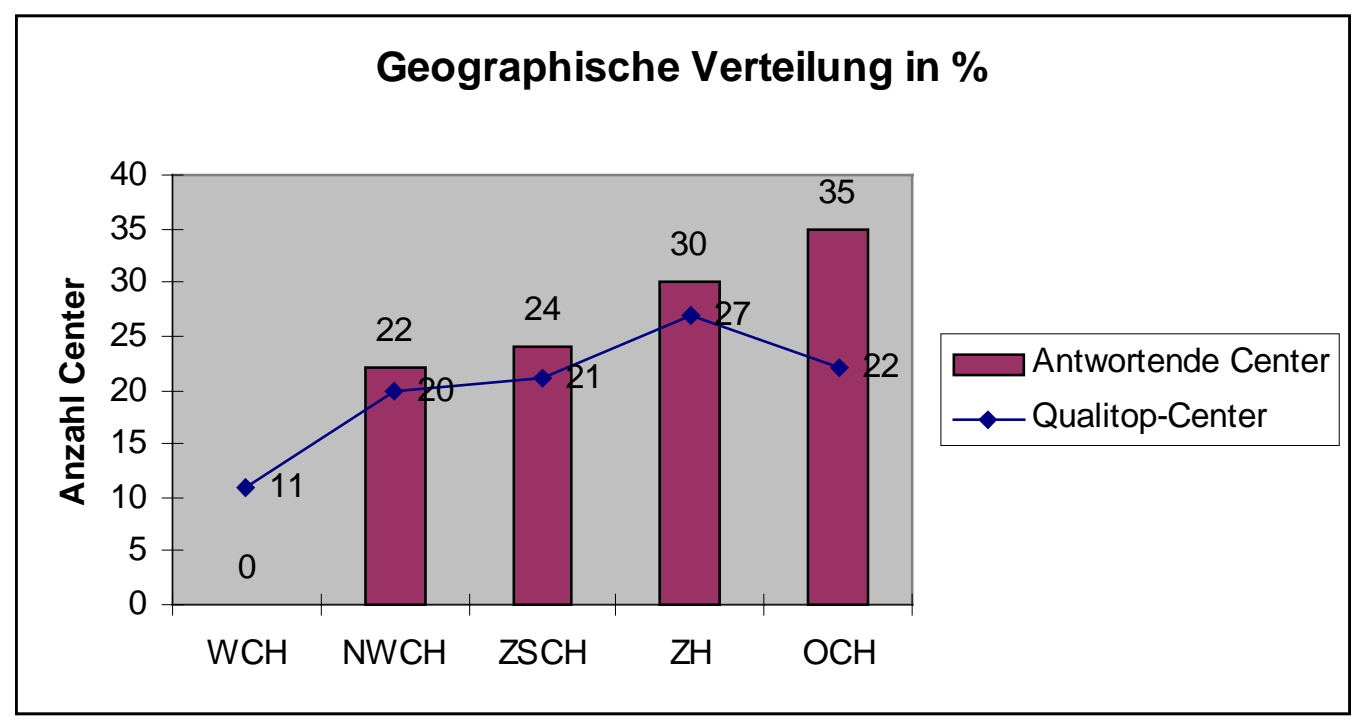

Abbildung 9-2 Geographische Verteilung der Center in \% 
Die antwortende Stichprobe kann von der geographischen Verteilung her mit Ausnahme der Westschweiz als repräsentativ für die Gesamtmenge der qualitop-zertifizierten Center in der Schweiz bezeichnet werden.

Die Westschweiz ist in der Studie nicht vertreten, weil der Fragebogen nur in deutscher Sprache verfasst war. Die Welschen, die auf sprachliche Diskriminierung sehr empfindlich reagieren, haben sich offensichtlich geweigert, an der Umfrage teilzunehmen. Der Tessin als italienisch sprechendes Gebiet der Schweiz ist in der Untersuchung mit zwei der insgesamt sechs Center vertreten.

- Die Stichprobe der antwortenden Center ist für die deutsche und die italienische Schweiz repräsentativ.

\subsection{Flächenmässige Verteilung der Center}

Die flächenmässige Verteilung der Center präsentiert sich folgendermassen:

\begin{tabular}{|l|r|r|}
\hline Fläche & Antwortende Center & Qualitop-Center \\
\hline $0-200 \mathrm{~m}^{2}$ & $6 \%$ & $29 \%$ \\
\hline $0-300 \mathrm{~m}^{2}$ & $8 \%$ & $28 \%$ \\
\hline $301-560 \mathrm{~m}^{2}$ & $33 \%$ & $36 \%$ \\
\hline$>560 \mathrm{~m}^{2}$ & $53 \%$ & $8 \%$ \\
\hline
\end{tabular}

Tabelle 9-2 Flächenmässige Verteilung der Center

Die Zahlen für alle Qualitop-Center stammen von QUALITOP, 1999.

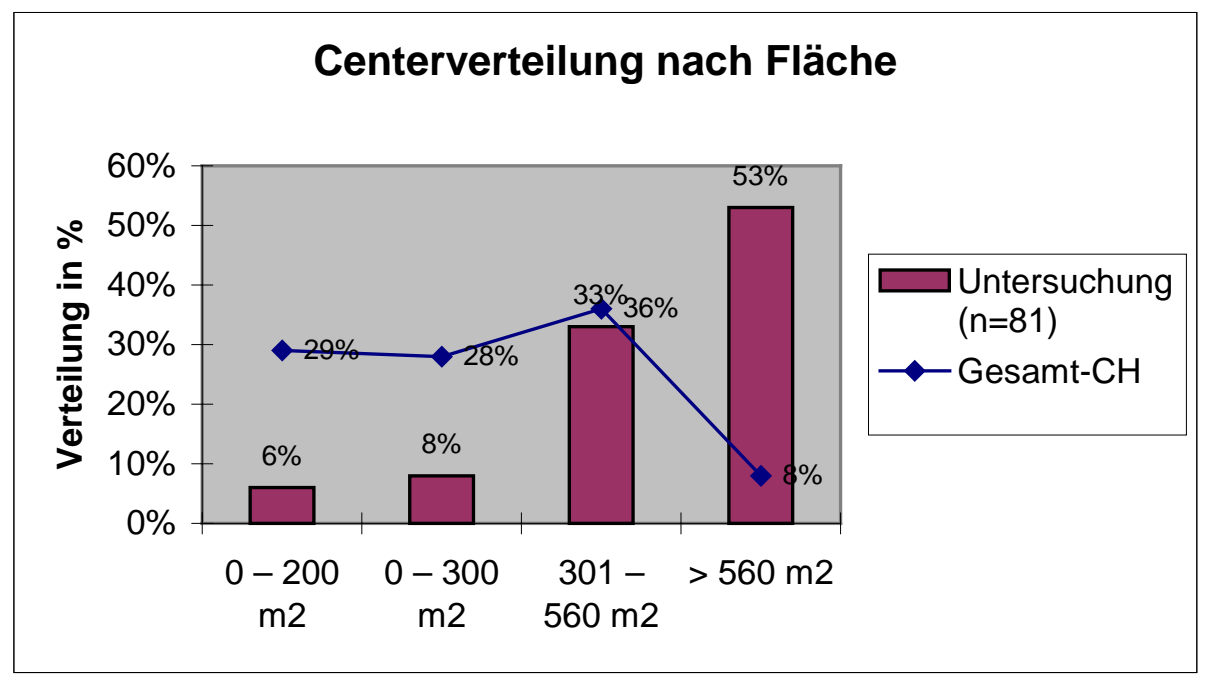

Abbildung 9-3 Flächenmässige Verteilung der Center 
Die beiden Stichproben entsprechen sich sehr wenig. Die kleinen Center $\left(0-300 \mathrm{~m}^{2}\right)$ sind stark untervertreten, die grossen Center ( über $560 \mathrm{~m}^{2}$ ) hingegen sind sehr stark übervertreten.

Die kleinen Center haben sich vielleicht aus Zeitmangel nicht beteiligt (Centerleiter ist nebenbei auch noch Trainingsinstruktor, bzw. ein Trainer ist nebenbei noch Geschäftsführer...) oder sind an den Fragestellungen der Untersuchung nicht interessiert. Die grossen Center (über $560 \mathrm{~m}^{2}$ ) sind nicht mehr einfach so nebenbei zu führen. Hier ist der Geschäftsführer wahrscheinlich fast ausschliesslich Manager und nicht mehr auch noch als Trainer oder Instruktor tätig. In dieser Untersuchung steht ja gerade die Person des Managers im Zentrum des Interessens und nicht die des Trainers. In diesem Sinne ist die Übervertretung der grossen Center zu Lasten einer Untervertretung der kleinen Center erfreulich.

- Flächenmässig kann die Stichprobe nicht als repräsentativ bezeichnet werden. Im Sinne dieser Untersuchung, wo der Manager (und nicht der Trainer) im Zentrum des Interessens steht, ist die Untervertretung der kleinen Center (Leiter eher auch noch Trainer) und die Übervertretung der grossen Center (Leiter v.a. Manager) aber eher als positiv für die Aussagekraft der Antworten zu werten.

\subsection{Geschlechtsverteilung der Centerleitenden}

Die Geschlechterverteilung der Center-Leitenden ist die folgende:

\begin{tabular}{|l|l|l|l|l|}
\hline Geschlecht & \multicolumn{3}{l|}{ Qualitop-Center } & \multicolumn{2}{l|}{ Antwortende Center } \\
\hline & Anzahl & In $\%$ & Anzahl & In \% \\
\hline Weibliche Leiterinnen & 16 & 7.7 & 6 & 7.4 \\
\hline Männliche Leiter & 191 & 92.3 & 75 & 92.6 \\
\hline Total & $\mathbf{2 0 7}$ & $\mathbf{1 0 0}$ & $\mathbf{8 1}$ & $\mathbf{1 0 0}$ \\
\hline
\end{tabular}

Tabelle 9-3 Geschlechter-Verteilung der Center-Leitenden

Die Zahlen sind für die Fitness-Frauen relativ ernüchternd. Im Aerobic- und Gymnastikbereich der Fitnesscenter, die wahrscheinlich fast die Hälfte des Umsatzes eines Fitnesscenters ausmachen, sind ein sehr grosser Teil der Instruktoren weiblichen Geschlechts. Im Management jedoch sind die Frauen krass untervertreten.

Es zeigt sich ein ähnlicher Effekt wie in den Schulen, wo ebenfalls ein grosser Anteil Frauen unterrichtet, die Oberlehrerstellen und Rektorenstellen aber fast ausnahmslos mit Männern besetzt sind. In Zukunft wird sich hier sicher noch etwas verändern; momentan ist die Fitnessbranche, was das Management angeht, aber immer noch sehr klar Männerdominiert. 
Die nur sechs Frauen, die an der Untersuchung teilgenommen haben, sind - obwohl sie der gesamtschweizerischen Geschlechterverteilung sehr gut entsprechen - leider zu wenig, als dass gewisse Fragen im Auswertungsteil (vergleiche Kapitel Fehler! \begin{tabular}{|l|l|l|}
\hline Verweisquelle konnte nicht gefunden werden. Fehler! Verweisquelle konnte nicht \\
\hline
\end{tabular} gefunden werden. $\mathrm{ab}$ Seite Fehler! Textmarke nicht definiert.) geschlechterspezifisch ausgewertet werden könnten. Dieser Geschlechtsvergleich wäre sehr interessant, ist aber mit dieser Stichprobe nicht möglich und müsste in einer anderen Arbeit untersucht werden.

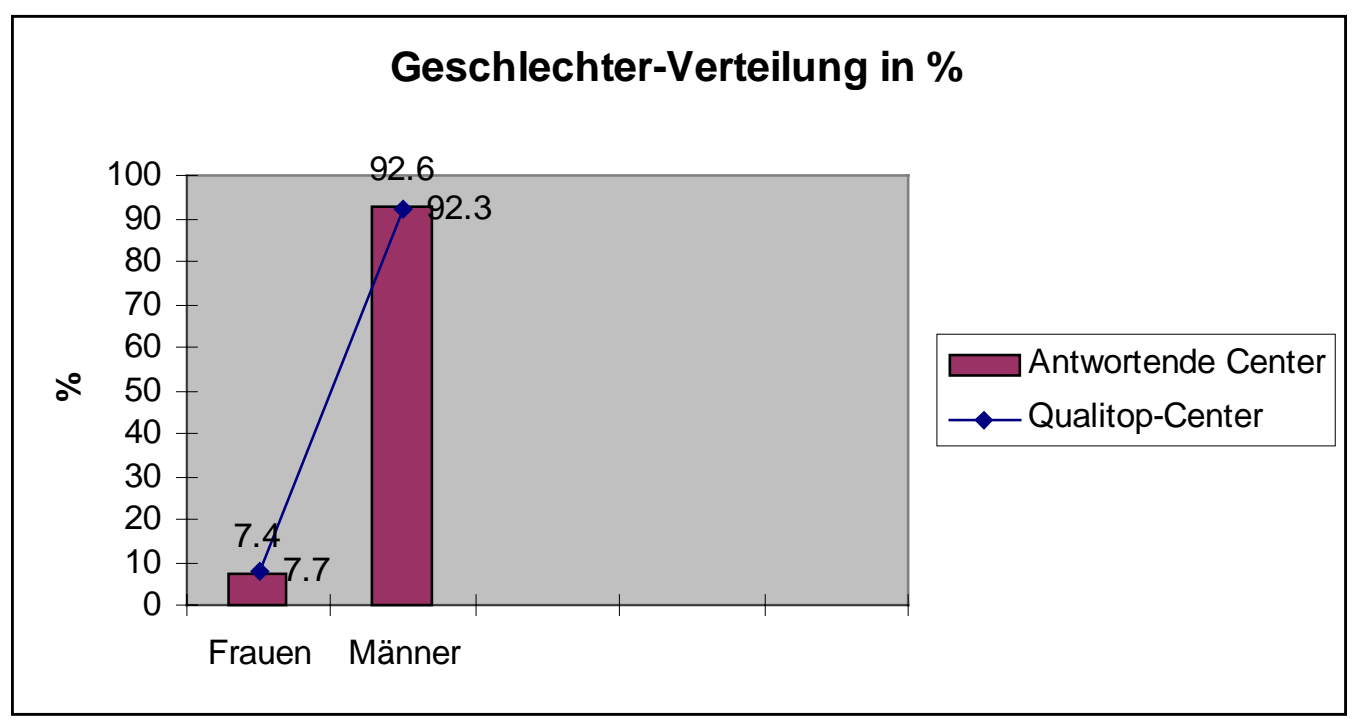

Abbildung 9-4 Geschlechterverteilung der Center-Leitenden

- Von der Geschlechterverteilung her kann die Stichprobe als repräsentativ bezeichnet werden.

\subsection{Validitätsprüfung anhand der Konkurrenzsituation}

Die Konkurrenzsituation wurde in der Untersuchung mit einer Frage nach der subjektiven Einschätzung durch die Centerleitenden erhoben:

„Wie gross beurteilen Sie den Konkurrenzdruck für Ihr Center?

$O$ klein $O$ erträglich $O$ gross $O$ sehr gross“

Zusätzlich zu den subjektiv erhobenen Daten wurde eine „objektive“ Konkurrenzsituation erhoben. Dabei wurde wie folgt vorgegangen:

Alle Qualitop-Center der Schweiz wurden auf einer Landeskarte eingezeichnet. Auf jedes Center wurde eine Folie mit einem eingezeichneten Radius von $20 \mathrm{~km}$ gelegt und die darin enthaltenen Konkurrenzcenter gezählt. Lag ein See oder ein Hügel zwischen 
den Centern, wurde nicht die Luftlinie berücksichtigt, sondern die effektive Fahrdistanz um das jeweilige Hindernis herum.

Dies ergab Werte zwischen 1 - 21 Centern, die im Umkreis von $20 \mathrm{~km}$ des jeweiligen Fitnesscenters lagen.

Anschliessend wurden die persönlichen Einschätzungen mit der gemessenen Konkurrenzsituation nach SPEARMAN korreliert. Resultat: $r=0.224, p=0.062(>0.05)$.

Die Zusammenhänge zwischen persönlicher Einschätzung des Konkurrenzdruckes und gemessener Konkurrenzsituation sind also nicht ganz signifikant, haben aber eine starke Tendenz zur Signifikanz.

Als mögliche Erklärungen für die Abweichungen von persönlicher Einschätzung und effektiv gemessener Fitnesscenterdichte können folgende Punkte angeführt werden:

\section{Center, die nicht QUALITOP zertifiziert sind}

Center, die nicht QUALITOP zertifiziert sind, wurden in unserer Messung der Konkurrenzsituation nicht erfasst, sind aber in Realität und in der Wahrnehmung der Manager sehr wohl eine Konkurrenz.

\section{Nischen}

Je grösser die Fitnesscenterdichte ist, desto mehr müssen sich die einzelnen Center auf einzelne Bereiche spezialisieren und Nischen besetzen. Dadurch verringert sich jedoch der effektive Konkurrenzdruck wieder. Der durch die Centerleitenden wahrgenommene Konkurrenzdruck der Center mit mehr als 15 Konkurrenten innerhalb der $20 \mathrm{~km}$ (Konkurrenzdruckeinschätzung 3.1, 3 = gross, $4=$ sehr gross) ist denn auch nur wenig höher als derjenige der Centerleitendenden mit zwischen 5 und 10 Konkurrenz-Centern (2.9).

Ein ähnliches Ergebnis zeigt auch der Vergleich der Konkurrenzsituation zwischen Centern in der Stadt und auf dem Land (vergleiche Unterkapitel 19.1 Unterschiede Stadt - Land Seite 134. Obwohl es in den Städten mehr Center hat als auf dem Land, ist der Konkurrenzdruck in den Städten nicht signifikant höher als auf dem Land.

- Im Bereich der Konkurrenzsituation konnte eine gute Übereinstimmung von subjektiver Einschätzung durch die Centermanager und objektiver Messung festgestellt werden.

- Es kann davon ausgegangen werden, dass diese Übereinstimmung auch in den anderen Bereichen besteht.

- Die Untersuchung kann damit als valid bezeichnet werden. 


\section{Ergebnisse des Bausteins „Persönlichkeit“}

\subsection{Verteilung der 16 Persönlichkeitstypen}

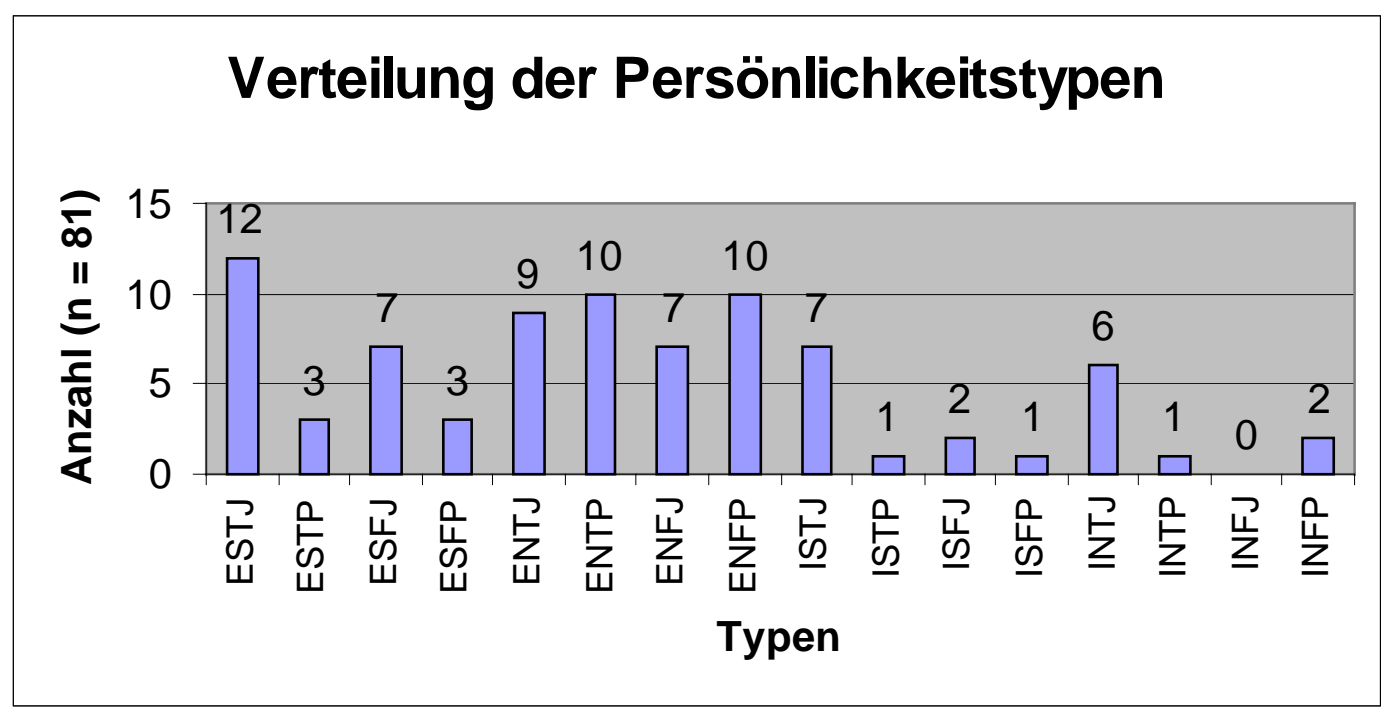

\section{Abbildung 10-1 Verteilung der Persönlichkeitstypen}

Man kann die Frage stellen, ob sich die verschiedenen Typen in der untersuchten Stichprobe gleich auf die verschiedenen Persönlichkeitstypen verteilen. Der Eindruck aus obiger Tabelle, dass keine Gleichverteilung vorliegt, wird bei einer Signifikanzprüfung mittels Chiquadrat-Test bestätigt (Chiquadrat $=44.8$; $\mathrm{df}=15, \mathrm{p}<0.001$ ). Es wird jedoch die Anwendungsvoraussetzung des Tests verletzt, dass nicht mehr als eine erwartete Zellhäufigkeit unter 5 sein darf (vgl. Schübo et al., 1991). Deshalb muss auf die Interpretation dieses Tests verzichtet werden. Man kann lediglich festhalten, dass die Typen ungleichmässig verteilt sind und ausser INFJ alle Typen in der Stichprobe vertreten sind.

\subsection{Verteilung auf die vier Ausprägungen E-I, N-S, T-F und J-P}

Konkretere Aussagen ergeben sich, wenn man die Verteilung auf die 4 Ausprägungen E-I, N-S, T-F und J-P anschaut.

\begin{tabular}{|l|l|}
\hline$E: I$ & $75: 25$ \\
\hline$N: S$ & $56: 44$ \\
\hline$T: F$ & $60: 40$ \\
\hline$J: P$ & $60: 40$ \\
\hline
\end{tabular}

Tabelle 12-I Verteilung der vier Ausprägungen 
Dies ergibt folgende graphische Darstellung:

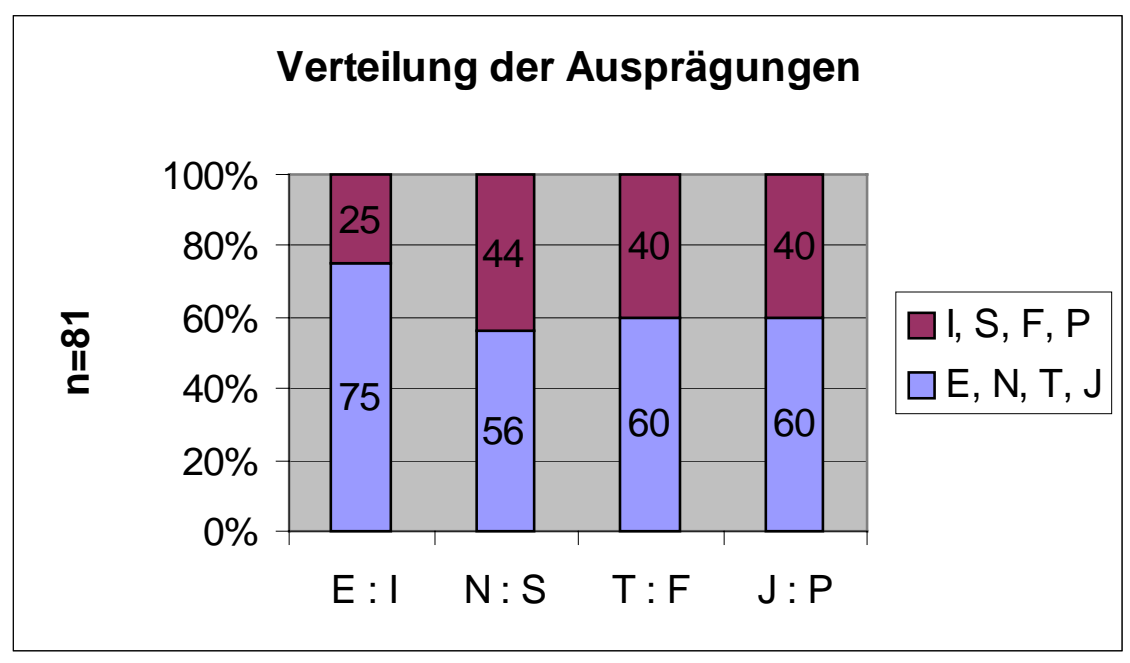

\section{Abbildung 10-2 Verteilung der Ausprägungen}

\section{Extraversion (E) versus Introversion (I)}

Die beiden Ausprägungen wurden schon im Theorieteil beschrieben (7.4.2 MyersBriggs Type Indicator, Seite 33), werden jedoch hier - damit nicht geblättert werden muss - der Einfachheit halber nochmals aufgelistet.

Der Extrovertierte (E)

- Ist ein guter Redner

- Liebt die Geselligkeit

- Geht von sich aus auf andere zu

- Will beständig die Welt verändern

- Verfügt über grosse Interessensbreite

- Tankt Energie im Kontakt mit anderen

- Beobachtet engagiert das äussere Geschehen
Der Introvertierte (I)

- Ist ein guter Zuhörer

- Schätzt das Alleinsein

- Wartet, dass man auf ihn zugeht

- Reflektiert die Welt

- Verfügt über grosse Interessenstiefe

- Schöpft Energie aus sich heraus

- Beobachtet eher das innere Geschehen

Drei Viertel aller Fitnesscenter-Manager, die an der Befragung teilgenommen haben, sind extrovertiert.

Dies erstaunt nicht, da sich eine offene und nach aussen gerichtete Persönlichkeit in Dienstleistungsbetrieben mit Kundenkontakt wohler fühlt als eine introvertierte.

Für die Kunden der persönliche Kontakt sehr wichtig ist (vergl. Abbildung 10-5 Wichtigkeit der situativen Variablen aus Kundensicht). Dies wiederum spricht eher für den Extrovertierten. Als Centerleiter steht man zwar weniger im direkten Kundenkontakt, sondern hat vermehrt Führungsfunktionen ,hinter den Kulissen“. Die Präsenz des Chefs an der Front wird aber von den Kunden stark geschätzt und ist deshalb auch nötig. Dazu kommt, dass man kaum direkt als Chef einsteigt, sondern sich über verschiedene Jobs in der Fitnessbranche zum Geschäftsleiter emporarbeitet. Auf diesem Weg ist Extrovertiertheit sicher sehr hilfreich. 


\section{Sensitive Wahrnehmung (S) versus intuitive Wahrnehmung (N) Analytische Entscheidung (T) versus gefühlsmässige Entscheidung (F) Geplanter Kontakt (J) mit der Aussenwelt versus spontaner Kontakt (P)}

Bei den andern drei Ausprägungen S-T, T-F und J-P kann keine ähnlich auffällige Verteilung wie bei der E-I-Ausprägung festgestellt werden. Die Verteilungen 60:40 und 56 : 44 können als ausgeglichen oder zumindest als nicht signifikant bezeichnet werden.

\subsubsection{Der Prototyp eines Fitnesscenter-Managers}

Statistisch gesehen könnte man also den ENTJ-Typ als den Prototyp eines FitnesscenterManagers bezeichnen. Dieser Typ ist in der vorliegenden Untersuchung auch am zweitmeisten vertreten. Der ENTJ-Typ ist wie folgt charakterisiert (MBTI-Manual, 1995):

Personen mit ENTJ-Präferenzen setzen ihre analytische Beurteilung ein, um möglichst alles in den Griff zu bekommen. Sie geben gern Anweisungen und planen langfristig. Da sie sich auf ihr analytisches Urteil verlassen, erscheinen sie logisch, objektiv kritisch und lassen sich meist nur durch vernünftige Argumente überzeugen. Ihre Aufmerksamkeit gilt vor allem den Ideen und nicht so sehr den Menschen, die Ideen einbringen.

Sie denken weit im voraus, planen Abläufe und einzelne Schritte für ein Projekt und gehen systematisch vor, um ihr Ziel termingerecht zu erreichen. Sie werden ungeduldig, wenn die Dinge unklar und nicht effizient sind und können hart sein, wenn es die Umstände verlangen.

Handlungen müssen folgerichtig und einsichtig sein, so denken sie, und handeln selbst danach. Ihr Weltbild und ihr eigenes Leben ist geordnet.

Sie interessieren sich vor allem für die Zukunft und überlegen, was man in der nächsten Zeit noch entwickeln könnte und geben sich weniger mit dem Bekannten ab oder mit dem, was jeder weiss und allen offensichtlich ist. Intuition befruchtet ihr Denken und macht sie empfänglich für neue Ideen und Theorien. Komplexe Probleme betrachten sie als Herausforderung.

Sie sind unzufrieden, wenn eine Arbeit ihren intuitiven Fähigkeiten nicht entspricht. Probleme stimulieren sie. Deshalb findet man diesen Typen häufig in Bereichen, in denen ständig neue Lösungsmöglichkeiten gefunden und umgesetzt werden müssen.

Diese Charakterisierung deckt sich sicher in vielen Bereichen mit den Anforderungen, die an einen erfolgreichen Manager oder Unternehmer - egal in welcher Branche gestellt werden:

- Logisch, objektiv kritisch, sachlich

- Delegieren gern

- Offen für Neues 
- Sehen die grossen Linien und Zusammenhänge

- Planen voraus

- Können hart sein

- Sehen Probleme als Herausforderung

Trotz dieser einsichtigen Übereinstimmung der Typenbeschreibung und den Anforderungen an eine erfolgreiche Managerin darf man nun nicht der Idee verfallen, nur ENTJ-Typen seien in der Fitnessbranche erfolgreich. Diese Typenzuteilung zeigt ja nur die Präferenzen einer Person. Die anderen Ausprägungen können ja ebenfalls aktiviert werden. Das situative Einsetzen der verschiedenen Ausprägungen je nach Situation im Wissen um die eigenen Präferenzen kann denn auch als eigentliche Reife bezeichnet werden. Dies bedeutet, dass auch ein Introvertierter I-Typ in der Branche absolut erfolgreich sein kann, wenn er es schafft, in gewissen Situationen, wo es nötig ist, seine E-Seite zu aktivieren.

Der erwähnte ENTJ-Prototyp des Fitnesscenter-Managers hat in dem Sinn einfach die beste Startposition. Ihm fällt das unternehmerische Handeln am Leichtesten. Die anderen Typen können dies jedoch durch bewusstes Entwickeln der eher unterentwickelten Persönlichkeitsaspekte kompensieren. Oder sie können sich für ihr Team entsprechend anders gelagerte Typen aussuchen, um damit die Defizite abzuschwächen.

Eine Typenuntersuchung von 834 Teilnehmern am Wirtschaftsforum für Führungskräfte (BENTS und BLANK 1995) zeigt folgende Typenverteilung:

\begin{tabular}{|l|l|}
\hline Typen & Prozentanteil \\
\hline ENTJ & $20 \%$ \\
\hline ESTJ & $17 \%$ \\
\hline ISTJ & $11 \%$ \\
\hline ENFJ & $10 \%$ \\
\hline ENFP & $8 \%$ \\
\hline ESFJ & $5 \%$ \\
\hline ENTP, INTJ & $7 \%$ \\
\hline INFP, INFJ & $3 \%$ \\
\hline ISFJ, INTP, ESTP & $2 \%$ \\
\hline ISTP, ISFP, ESFP & $1 \%$ \\
\hline
\end{tabular}

\section{Tabelle 12-II Typenverteilung von Führungskräften}

Auch hier ist der ENTJ-Typ am meisten vertreten. Offensichtlich bringen ENTJ-Typen viele Voraussetzungen mit, die für Führungsfunktionen nötig sind.

Grundsätzlich lässt sich eine recht gute Übereinstimmung der Typenverteilung zwischen unserer Stichprobe von Fitnesscenter-Managern und der Untersuchung von BENTS und BLANK (1995) beobachten (vergleiche Abbildung 12-3 Verteilung der Persönlichkeitstypen im Vergleich, Seite 69). Der durchgeführte Chiquadratanpassungs- 
test bestätigt dies (Chiquadrat $=16.1$; $\mathrm{df}=15, \mathrm{p}=.375$ ). Aus methodischen Gründen (Verletzung der Anwendungsvoraussetzungen in Bezug auf Zellhäufigkeiten) empfiehlt es sich, die Signifikanzprüfung nicht zu interpretieren.

Die grössten Unterschiede sind bei den ENTJ- und ENTP-Typen festzustellen. Im Fitness-Management treffen wir weniger ENTJ-Typen, dafür mehr ENTP-Typen wie grundsätzlich im Management.

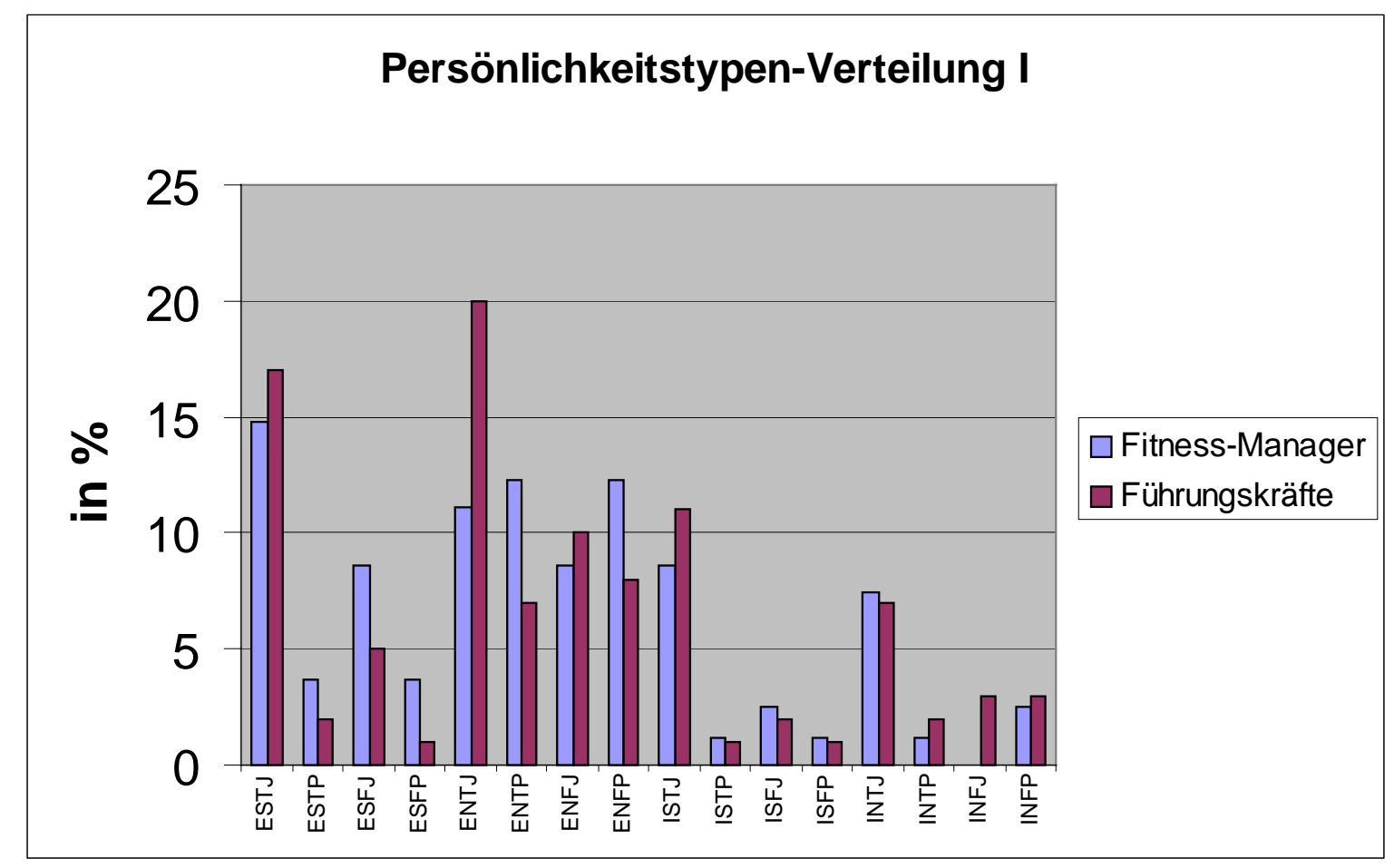

Abbildung 10-3 Verteilung der Persönlichkeitstypen im Vergleich I 
In der folgenden Abbildung wird die Typenverteilung im Betriebswirtschaftsstudium (BENTS und BLANK 1995, n=77), dem klassischen Studium für Führungskräfte, mit der Typenverteilung der effektiven Führungskräfte (BENTS und BLANK 1995) verglichen. Es bestätigt sich die obige Aussage, dass ENTJ-Typen wahrscheinlich grundsätzlich bereits viele Eigenschaften mitbringen, die für Führungsaufgaben wichtig sind.

Von den Studierenden, die eine Management-Ausbildung absolvieren, kommen, überproportional viele ENTJ-Typen „,weiter“ und bekleiden später Führungsfunktionen. Der Anteil von ENTJ-Typen beträgt bei den Studierenden $4 \%$ nimmt bei den Führungskräften auf $20 \%$ zu.

ESTJ-Typen und ISTJ-Typen sind im Vergleich zur Ausgangslage bei den Studierenden ebenfalls übervertreten, aber um einiges weniger markant als die ENTJ-Typen.

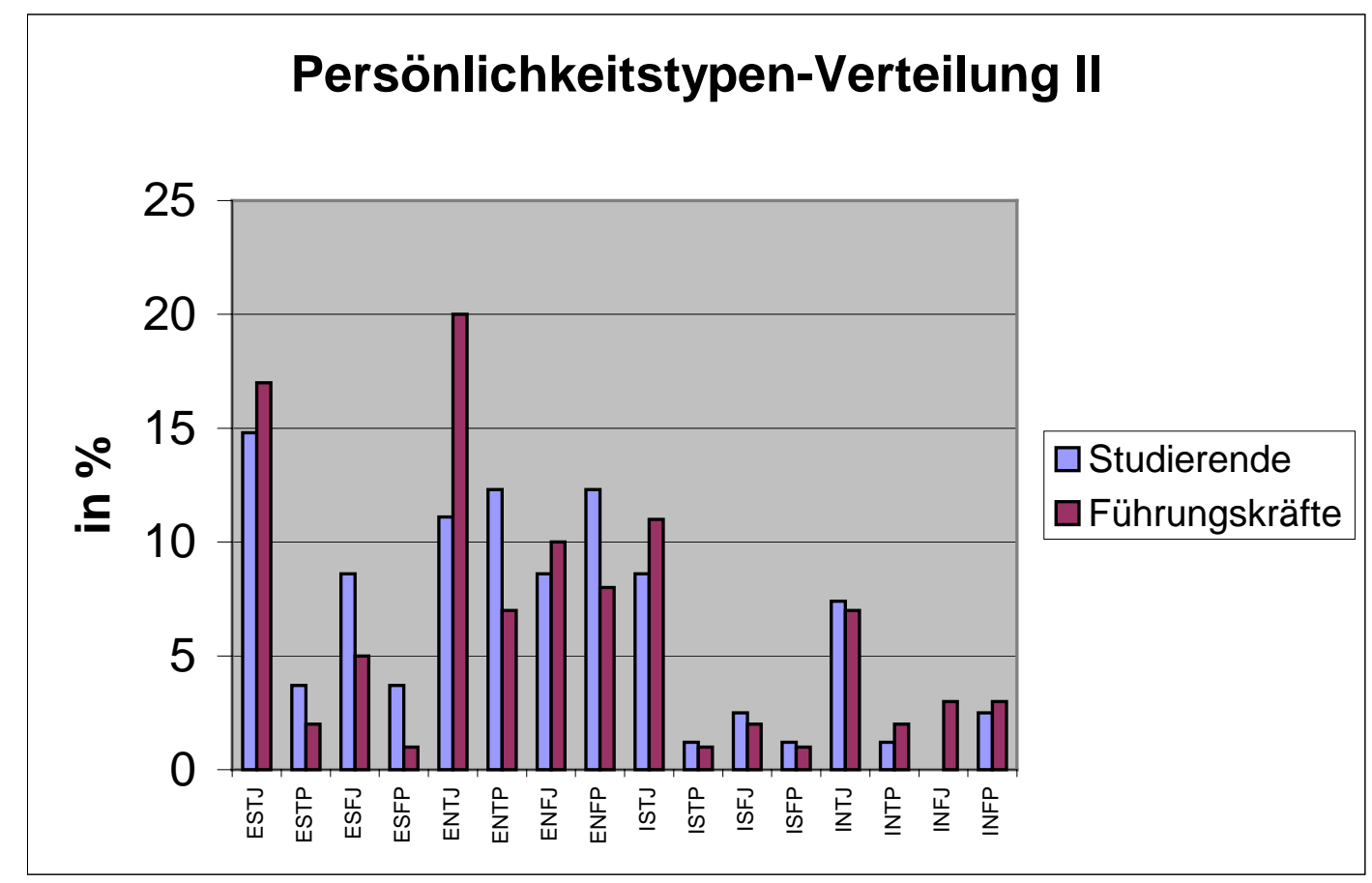

Abbildung 10-4 Verteilung der Persönlichkeitstypen im Vergleich II 
Die obigen Aussagen beziehen sich aber nur auf die Häufigkeit der Typen. Damit ist noch nichts über deren Erfolg ausgesagt.

Wie die Erfolgsquote der einzelnen Typen ist, werden wir im Unterkapitel „Zusammenhang von Persönlichkeit und Erfolg“ sehen. Einige Facts seien hier jedoch vorweggenommen:

- Die drei erfolgreichsten Manager der Untersuchung sind allesamt ESTJ-Typen.

- Die drei im Durchschnitt erfolgreichsten Typen sind ESTP, ESTJ und ESFP.

- Der Prototyp ENTJ liegt in der vorliegenden Untersuchung auf Platz sechs der Erfolgstypen, jedoch nur 7\% hinter dem Spitzenreiter.

\subsubsection{Der Gegentyp zum Prototyp}

Aus den ähnlichen Überlegungen wie oben lässt sich auch der klassische Anti-FitnessManager bezeichnen. Aufgrund der Statistik wäre es der ISFP-Typ. Er ist in unserer Untersuchung mit nur einem Vertreter repräsentiert. Bei den Untersuchungen von BENTS und BLANK ist er ebenfalls sehr schlecht vertreten ( $2 \%$ bei den Studierenden, $1 \%$ bei den Führungskräften).

Der ISFP-Typ wird im MBTI-Manual wie folgt charakterisiert:

Personen mit ISFP-Präferenzen vermitteln persönliche Wärme. Bisweilen zeigen sie diese warme Seite erst, wenn sie einen Menschen gut kennen - sie ist nach innen gewandt, wie das Innenfutter eines Mantels. Wenn sie sich jemandem zuwenden, dann tun sie es ganz - zeigen ihre Zuwendung jedoch eher durch Tun als durch Worte. Wenn sie von einer Sache überzeugt sind oder eine positive Beziehung $\mathrm{zu}$ einem Menschen haben, erfüllen sie die damit verbundenen Aufgaben und Verpflichtungen äusserst zuverlässig.

Sie sehen alles aus einer persönlichen Sicht. Massstab sind die eigenen Ideale und persönlichen Ansichten. Sie halten leidenschaftlich an ihren Überzeugungen fest, lassen sich aber durchaus von jemand beeinflussen, zu dem sie eine persönliche Beziehung haben. Obwohl innere Normen und Ideale für sie so grundsätzlich sind, finden sie es schwierig, darüber zu reden. Sie sprechen selten über ihre innersten Empfindungen; ihre Verletzlichkeit schützen sie durch reservierte Zurückhaltung. Im Alltäglichen sind sie tolerant, offen, flexibel und anpassungsfähig. Wenn man jedoch ihre Normen antastet, lassen sie sich auf keinen Kompromiss ein. Gewöhnlich geniessen sie den Augenblick und lassen sich diesen Genuss ungern dadurch verderben, dass sie unter Druck eine Terminsache abschliessen müssen. Sie haben keine Ambitionen, jemanden zu beeindrucken oder Macht auszuüben. Besonders schätzen sie Menschen, die sich Zeit nehmen, ihre Vorstellungen und Ziele zu verstehen.

Interessiert sind sie an der Wirklichkeit, wie sie über ihre fünf Sinne wahrnehmen. Sie fühlen sich in Bereichen $\mathrm{zu}$ Hause, in denen ihr Geschmack, ihre Wahrnehmungsfähigkeit und ihr Sinn für Schönheit und Formen angesprochen werden. Manche haben auch ein besonderes Verhältnis zur Natur oder zu Tieren. Oft betätigen sie sich erfolgreich im künstlerischen Bereich. Mit ihren Händen können sie sich meist besser ausdrücken als mit Worten. 
Diese Charakterisierung legt einzelne Verhaltensweisen offen, die für ManagementAufgaben sicher nicht unbedingt erfolgversprechend sind:

- Persönliche Sichtweise

- Fast stures Festhalten an eigenen Idealen und Vorstellungen

- Im Moment leben, wenig Verständnis für Terminangelegenheiten

- Wenig Ambitionen

- Drücken sich besser durch Tun aus als durch Worte

Auf der Liste der Erfolgstypen figuriert der ISFP-Typ bei den 15 in der Untersuchung vorhandenen Typen auf Rang 13, also an drittletzter Stelle. Wieder kann hier eine Tendenz bestätigt werden, eine statistisch abgestützte Aussage kann jedoch nicht gemacht werden.

\subsection{Verteilung der vier Kerntypen ST, SF, NT und NF}

Die prozentuale Verteilung der vier Kerntypen (vergl. 5.5 Die verschiedenen Typen des MBTI Seite 33 ergibt folgendes Ergebnis:

\begin{tabular}{|l|r|}
\hline ST & $28.5 \%$ \\
\hline SF & $16.0 \%$ \\
\hline NT & $32.0 \%$ \\
\hline NF & $23.5 \%$ \\
\hline
\end{tabular}

Tabelle 10-1I Verteilung der vier Kerntypen

Dies ergibt folgende graphische Darstellung:

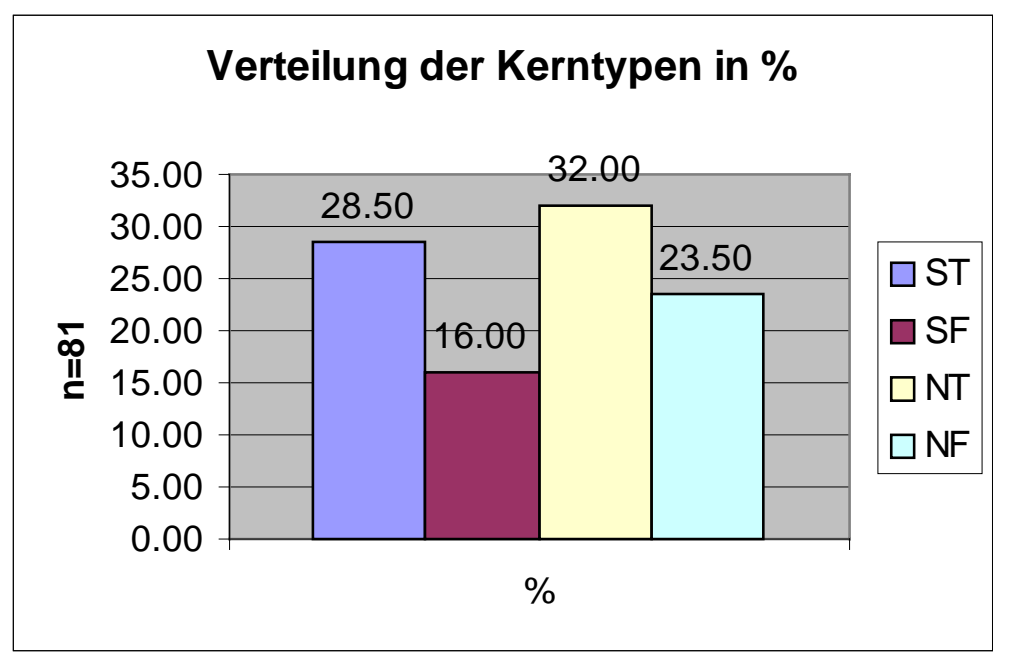

\section{Abbildung 10-5 Verteilung der Kerntypen}


Spitzenreiter ist der NT-Typ mit 32\%. Er wurde im Theorieteil (vergl. 5.5 Die verschiedenen Typen des MBTI Seite 33 wie folgt charakterisiert:

Der NT-Typ: der zielverliebte Visionär

- Nimmt Informationen intuitiv auf und entscheidet vom Kopf her

- Sieht das Ziel und die grossen Linien

- Guter Komplexitätsbewältiger

- Hasst Routinearbeiten

- Wirkt nach aussen kühl und distanziert

- Legt sich tendenziell mit Autoritäten an

- Scheut keinen Konflikt, bringt Kritik eher global denn konkret

Der NT-Typ kann als Prototyp des Unternehmers bezeichnet werden: zielorientiert, sieht die grossen Linien, entscheidet analytisch, ist konfliktfähig und lässt sich von Hindernissen (und auch Autoritäten) nicht von seinem Weg abbringen.

Es ist deshalb nicht erstaunlich, dass dieser Typ obenauf schwingt. Gerade in der sich permanent stark wandelnden Fitnessbranche muss man Informationen intuitiv schnell aufnehmen können, die Vision nicht aus den Augen verlieren und sich nicht in den Details verlieren. Diese Eigenschaften sind jedoch nicht nur in der Fitnessbranche gefragt, sondern in allen Branchen. Der Vergleich mit der Typenverteilung der Untersuchung von BENTS und BLANK bestätigt dies.

\begin{tabular}{|l|r|r|}
\hline Typ & SCHMID & BENTS \& BLANK \\
\hline ST & $28.5 \%$ & $31 \%$ \\
\hline SF & $16.0 \%$ & $9 \%$ \\
\hline NT & $32.0 \%$ & $36 \%$ \\
\hline NF & $23.5 \%$ & $24 \%$ \\
\hline
\end{tabular}

Tabelle 10-2 Vergleich der Verteilung der Kerntypen

Im Bereich der Auswertung des Zusammenhangs von Persönlichkeit und Erfolg werden wir sehen, ob der NT-Typ nicht nur übervertreten, sondern auch besonders erfolgreich ist.

Am schlechtesten vertreten ist der „Gegentyp“ zum NT-Typ, der SF-Typ mit 16\%. Der SF-Typ wurde wie folgt charakterisiert:

Der SF-Typ: der einfühlsame Praktiker

- Verlässt sich auf seine fünf Sinne und pflegt Beziehungen aktiv.

- Arbeitet gewissenhaft, konzentriert sich auf Tatsachen und Fakten

- Arbeitet lieber im Team und ist sehr hilfsbereit

- Engagiert sich weniger um der Aufgabe als um der Kollegen willen

- Nimmt Kritik persönlich und geht Konflikten aus dem Weg 
Einzelne Eigenschaften wie Kontaktpflege, gewissenhaftes Arbeiten und Hilfsbereitschaft sind im Dienstleistungssektor sehr wichtig und eine gute Voraussetzung für Erfolg. Als Chef sind jedoch Eigenschaften wie fehlende Konfliktfähigkeit und zu starke Beziehungsorientierung zu den Mitarbeitern suboptimal. Es erstaunt deshalb eigentlich auch hier nicht, dass dieser Typ untervertreten ist. Ob er aber effektiv weniger erfolgreich ist, wird sich im Kapitel 16 Zusammenhang zwischen Persönlichkeit und Erfolg(Seite 122, zeigen.

Die beiden anderen Kerntypen ST und NF siedeln sich mit $28.5 \%$ bzw. $23.5 \%$ zwischen den beiden ,Extrempolen“ an.

\subsection{Zusammenfassung „Persönlichkeit“}

- Unregelmässige Verteilung der Einzeltypen ohne Gesetzmässigkeit.

- Klare Dominanz der Extrovertierten (75\%) gegenüber den Introvertierten (25\%).

- T- und J-Typen etwa eineinhalb Mal so stark vertreten wie F- und P-Typ.

- Kerntyp NT doppelt so stark (32\%) vertreten wie „Gegentyp“ SF (16\%).

- Der ENTJ-Typ könnte als Prototyp des Fitnesscenter-Managers bezeichnet werden.

- Die Typenverteilung der Fitnesscenter-Manager entspricht der Typenverteilung des Berufstandes ,Manager“ recht gut. 


\section{Ergebnisse des Bausteins „Führungsverhalten“}

\subsection{Wichtigkeit der Führungstätigkeiten für den Erfolg}

Auf die Frage, welche Führungstätigkeiten für den Erfolg eines Fitnesscenters wichtig sind, wurden folgende Antworten erteilt:

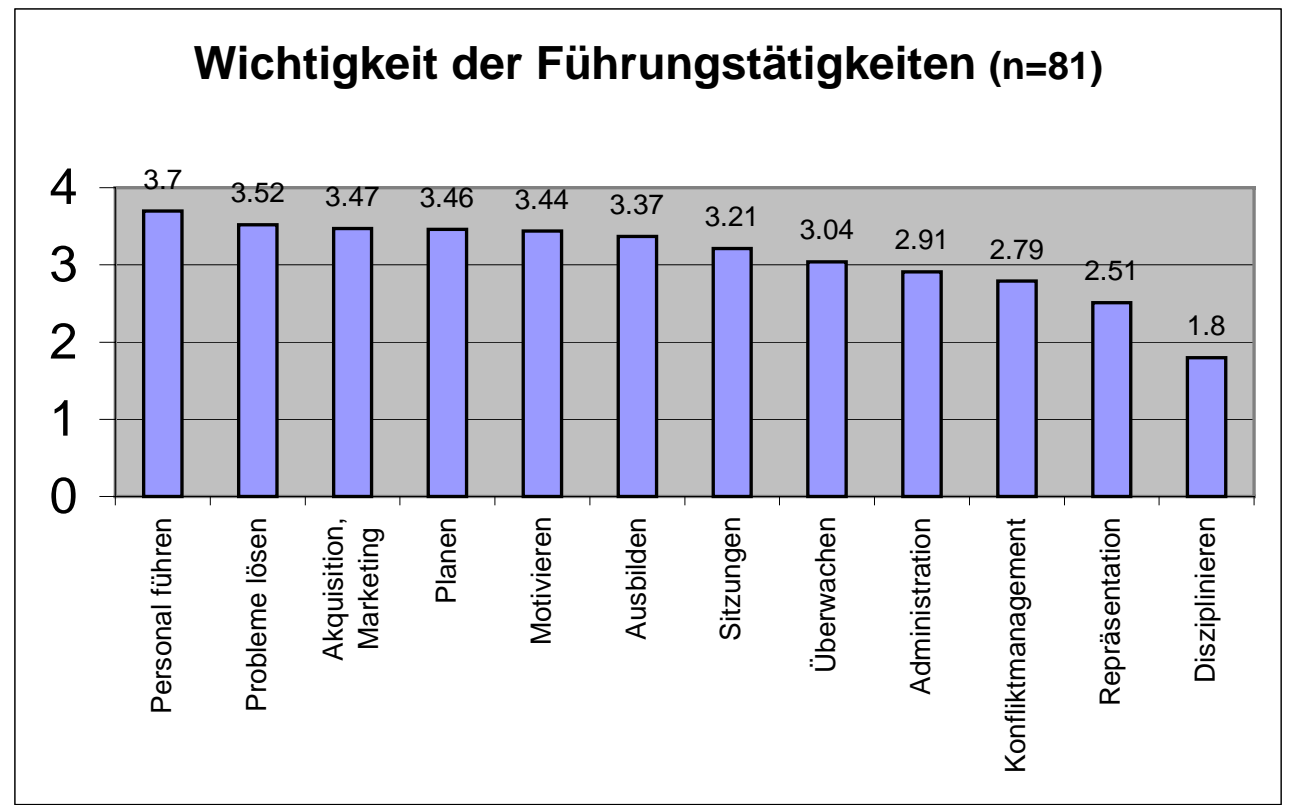

Legende:

$4=$ sehr wichtig, $3=$ wichtig, $2=$ ziemlich wichtig, $1=$ unwichtig

Durchschnittsrating von 81 Fitnesscentermanager, sortiert nach Wichtigkeit.

\section{Abbildung 11-1 Wichtigkeit der Führungstätigkeiten}

Detaillierte Beurteilung der Wichtigkeit der fünf wichtigsten Führungstätigkeiten:

\begin{tabular}{|l|l|l|l|l|}
\hline Tätigkeiten & Nennungen (\%) & & & \\
\hline & Sehr wichtig & Wichtig & Ziemlich wichtig & Unwichtig \\
\hline Personal führen & 75.3 & 19.8 & 4.9 & 0 \\
\hline Probleme lösen & 56.8 & 38.3 & 4.9 & 0 \\
\hline $\begin{array}{l}\text { Akquisition, } \\
\text { Marketing }\end{array}$ & 53.1 & 37.0 & 7.4 & 2.5 \\
\hline Planen & 58.0 & 29.6 & 10.5 & 1.7 \\
\hline Motivieren & 60.5 & 27.2 & 8.6 & 3.7 \\
\hline
\end{tabular}

Tabelle 11-1 Wichtigkeit der Führungstätigkeiten im Detail 
Diese Verteilung sieht graphisch dargestellt folgendermassen aus:

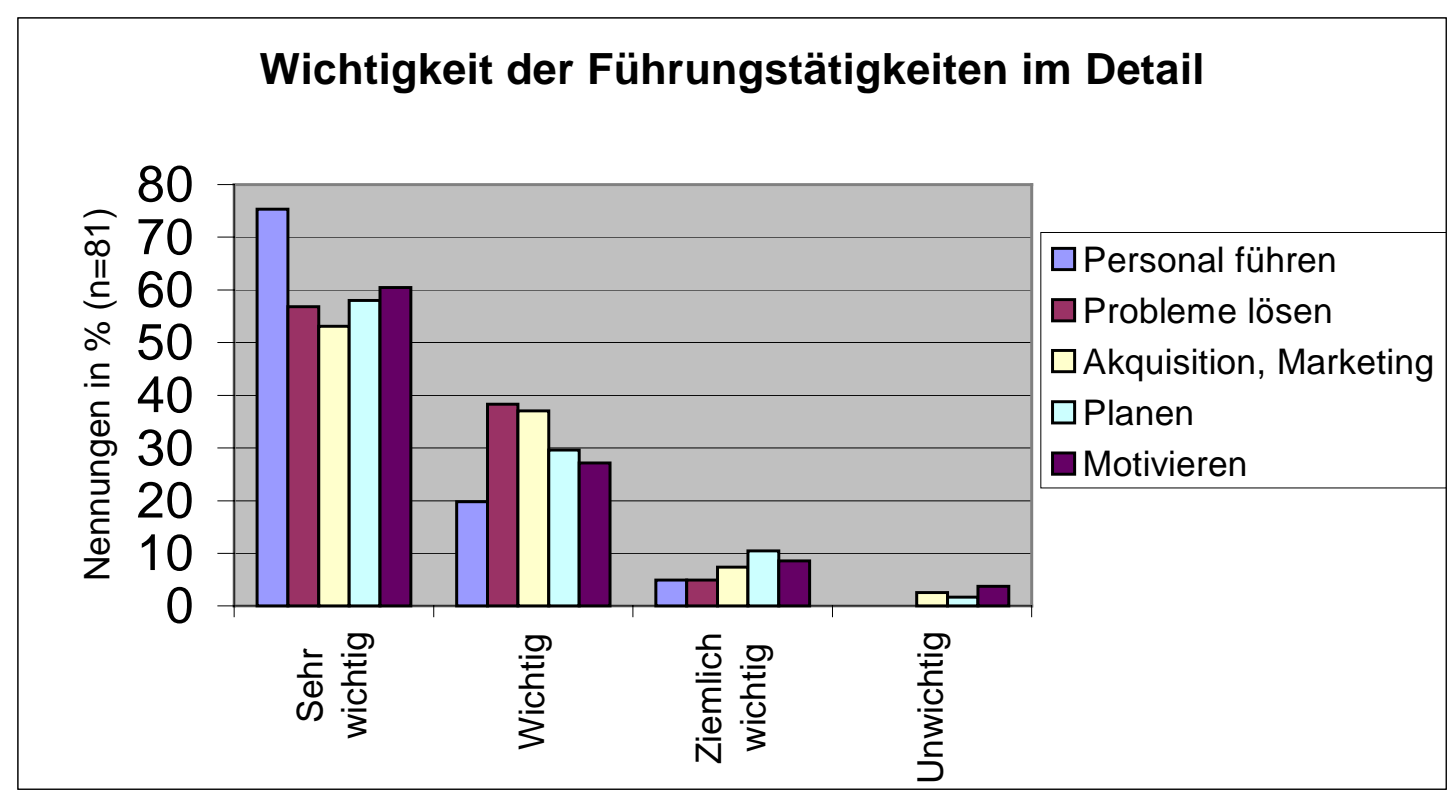

\section{Abbildung 11-2 Wichtigkeit der Führungstätigkeiten im Detail}

Sehr wichtig

Jeweils über 50\% der Befragten bezeichneten die fünf Führungstätigkeiten

- Personal führen

- Probleme lösen

- Akquisition, Marketing

- Planen

- Motivieren

als sehr wichtig. 
Am wichtigsten

Bei der Frage nach den wichtigsten zwei unter den sehr wichtigen Tätigkeiten ergab sich folgende Verteilung:

\begin{tabular}{|l|r|r|}
\hline Tätigkeit & \%-Stimmen & Rang \\
\hline Personal führen & & \\
\hline Planen, koordinieren & $40.8 \%$ & 1 \\
\hline Motivieren, bestärken & $30.9 \%$ & 2 \\
\hline Akquisition, Marketing & $25.9 \%$ & 3 \\
\hline Ausbilden & $24.7 \%$ & 4 \\
\hline Probleme lösen & $17.3 \%$ & 5 \\
\hline Sitzungen, Informationsaustausch & $16.0 \%$ & 6 \\
\hline Überwachen, kontrollieren & $13.5 \%$ & 7 \\
\hline Repräsentation, soziale Verpflichtungen & $8.6 \%$ & 8 \\
\hline Konfliktmanagement & $3.7 \%$ & 9 \\
\hline Administration, Papierarbeit & $2.5 \%$ & 10 \\
\hline Disziplinieren, bestrafen & $2.4 \%$ & 11 \\
\hline Keine Angabe & $0 \%$ & 12 \\
\hline
\end{tabular}

Tabelle 11-2 Die wichtigsten Führungstätigkeiten

Die wichtigste Führungstätigkeit aus der Sicht der befragten Manager ist „Personal führen“. Mit $41 \%$ oder etwa einem Fünftel aller Nennungen steht sie klar vor einer Dreiergruppe bestehend aus Planen/koordinieren, Motivieren/bestärken und Akquisition/Marketing mit jeweils 25 - 30\% aller Nennungen.

Der Befund, Personal führen sei die wichtigste Führungstätigkeit, widerspiegelt auch die Sicht der Kunden. Die beiden wichtigsten Kriterien aus Kundensicht sind Freundlichkeit des Personals und Kompetenz des Personals (vergl. 13.3 Erfolgsfaktoren aus Kundensicht Seite 101. Effektive Personalführung konzentriert sich folglich vor allem auf die beiden Aspekte Freundlichkeit und Kompetenz der Angestellten. 
Fach- oder Sozialkompetenz?

Die folgende Liste ist ein Versuch, zu beschreiben, inwiefern die verschiedenen Führungstätigkeiten eher Fachkompetenz voraussetzen oder vor allem Sozialkompetenz benötigen (erhoben im Rahmen der Interviews mit den Centerleitern, $n=3$ )

\begin{tabular}{|l|c|c|c|}
\hline Tätigkeit & Rang & $\begin{array}{c}\text { Fach- } \\
\text { kompetenz }\end{array}$ & $\begin{array}{c}\text { Sozial- } \\
\text { kompetenz }\end{array}$ \\
\hline & & & \\
\hline Personal führen & 1 & & $\mathrm{x}$ \\
\hline Planen, koordinieren & 2 & $\mathrm{x}$ & $(\mathrm{x})$ \\
\hline Motivieren, bestärken & 3 & & $\mathrm{x}$ \\
\hline Akquisition, Marketing & 4 & $\mathrm{x}$ & $(\mathrm{x})$ \\
\hline Ausbilden & 5 & $\mathrm{x}$ & $\mathrm{x}$ \\
\hline Probleme lösen & 6 & $(\mathrm{x})$ & $\mathrm{x}$ \\
\hline Sitzungen, Informationsaustausch & 7 & & $\mathrm{x}$ \\
\hline Überwachen, kontrollieren & 8 & $\mathrm{x}$ & \\
\hline $\begin{array}{l}\text { Repräsentation, } \\
\text { Verpflichtungen }\end{array}$ & 9 & & $\mathrm{x}$ \\
\hline Konfliktmanagement & 10 & & $\mathrm{x}$ \\
\hline Administration, Papierarbeit & 11 & $\mathrm{x}$ & \\
\hline Disziplinieren, bestrafen & 12 & & $\mathrm{x}$ \\
\hline
\end{tabular}

Tabelle 11-3 Führungstätigkeiten nach Fach- und Sozialkompetenz

Die Aufstellung zeigt, dass grundsätzlich im Führungsbereich die sozial-menschlichen Fähigkeiten eher gefordert sind, als die eigentliche Fachkompetenz. Grundsätzlich brauchen natürlich alle Führungstätigkeiten Fachkompetenz, um die Situation und die Auswirkungen des Führungsverhaltens beurteilen zu können. Das eigentliche Führen ist jedoch am Schluss eine Frage der Kommunikation und ohne die menschlich-soziale Komponente wird es niemandem gelingen, die vorhandene Sachkenntnis umzusetzen.

Es wird somit auch verständlich, warum ,grosse“ Führungspersönlichkeiten die Branche manchmal fast wechseln wie andere die Kleider. Ob CEO in einer Chemiefirma, in einem Diensleistungsunternehmen oder in einem Produktionsbetrieb: die eigentlichen Führungstätigkeiten bleiben sehr ähnlich. Die nötige Fachkompetenz oder das Spezialistenwissen kann durch die langjährigen Mitarbeiter eingebracht werden.

In der Fitnessbranche sind die sozialen Kompetenzen noch wichtiger als in anderen Branchen. Neben der eigentlichen Manager-Funktion sind die meisten Centerleiterinnen auch noch als Trainingsberaterin tätig oder pflegen anderweitig den persönlichen Kontakt zu den Kunden. Es ist deshalb erstaunlich, dass die herkömmlichen Weiterbildungsangebote für Fitnesscenter-Manager vor allem den Bereich Fachkompetenz abdecken. Im Bereich Sozial- und Selbstkompetenz bestehen wenig branchenspezifische Angebote. Ein Bedürfnis hierzu besteht jedoch. Die auf dem Antworttalon als Zusatz angebrachte Frage ,Sind Sie an Kursen zum Thema Persönlichkeits-Management interessiert?“" wurde von etwa 45\% der Befragten bejaht. 


\subsection{Zeitaufwand für die einzelnen Führungstätigkeiten}

Nicht alle Führungstätigkeiten nehmen gleich viel Zeit in Anspruch. Die folgende Grafik zeigt die zeitliche Belastung der einzelnen Tätigkeiten.

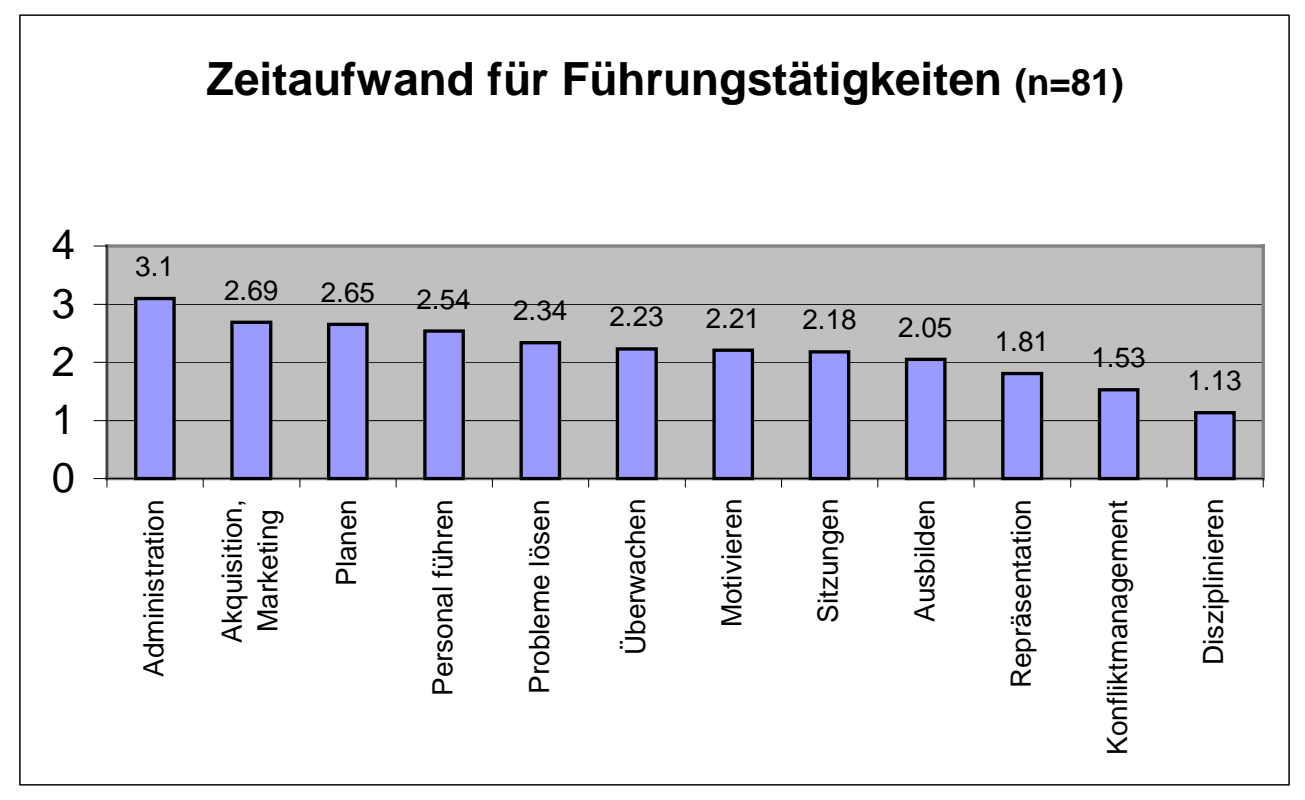

Legende:

$4=$ mehr als $6 \mathrm{~h}$ pro Woche, $3=4-6 \mathrm{~h}$ pro Woche, $2=1-3 \mathrm{~h}$ pro Woche, $1=$ weniger als $1 \mathrm{~h}$ pro Woche.

Durchschnittsrating von 81 Fitnesscenter-Manager, sortiert nach Zeitaufwand.

\section{Abbildung 11-3 Zeitaufwand für Führungstätigkeiten}

Mit etwas über $4-6 \mathrm{~h}$ pro Woche braucht Administration und Papierarbeit am meisten Zeit. $37 \%$ der Befragten gaben an, dass sie über $6 \mathrm{~h}$ pro Woche darin investierten, $39.5 \%$ kreuzten $4-6 \mathrm{~h}$ an.

Die Ergebnisse decken sich zu weiten Teilen mit den Aussagen von URSPRUNG (1998), der in seiner Studie über das Berufsfeld Fitnesscenter die Führungstätigkeiten des Fitnesscenter-Managers nach Zeitaufwand wie folgt beschreibt:

- Administrative Aufgaben wie Buchhaltung, Planung, Mitgliederadministration

- Marketing, Unternehmenskonzept und dessen Umsetzung

- Führung der Mitarbeitenden

- Arbeit an internen Dokumentationen, was wieder als Administration bezeichnet werden könnte. 


\subsection{Führungsverhalten: Wichtigkeit für den Erfolg und Zeitaufwand}

Interessant wird die Gegenüberstellung von Wichtigkeit der Führungstätigkeit und die dafür eingesetzte Zeit.

Es zeigt sich, dass die beiden Parameter nicht in direkter Abhängigkeit zueinander stehen: mit abnehmender Wichtigkeit wird nicht unbedingt weniger Zeit eingesetzt.

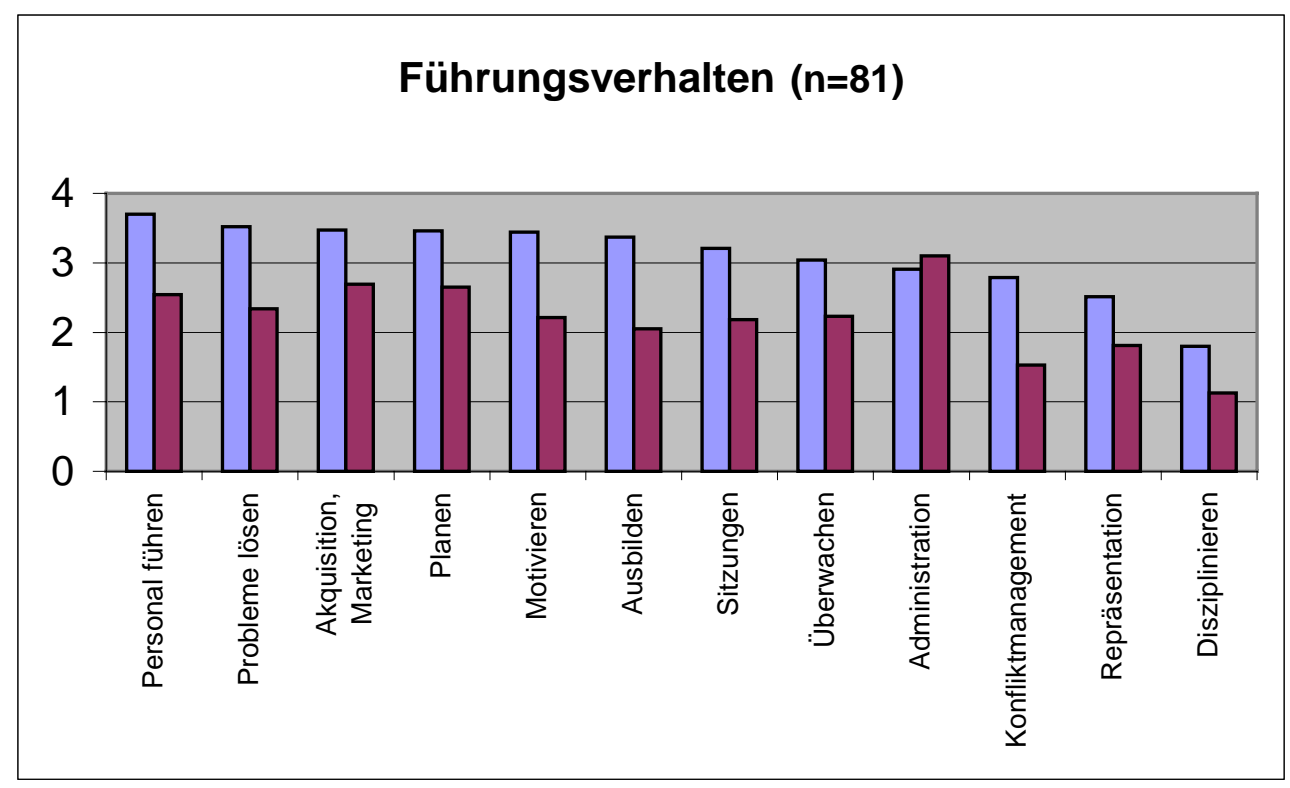

Legende:

Kolonne links: Wichtigkeit $(4=$ sehr wichtig, $3=$ wichtig, $2=$ ziemlich wichtig, $1=$ unwichtig)

Kolonne rechts: Zeitaufwand $(4=$ mehr als $6 \mathrm{~h}$ pro Woche, $3=4-6 \mathrm{~h}$ pro Woche, $2=1-3 \mathrm{~h}$ pro Woche, $1=$ weniger als $1 \mathrm{~h}$ pro Woche).

\section{Abbildung 11-4 Führungsverhalten}

Es fällt auf, dass Administration/Papierarbeit mit Abstand die meiste Zeit in Anspruch nimmt. Von der Wichtigkeit für den Erfolg her steht diese Tätigkeit jedoch erst an neunter Stelle.

Die Auswertung zeigt klar, dass die persönliche Arbeitstechnik von einigen FitnessManagern noch verbessert werden kann. Auch dieser Bereich ist mit dem bestehenden Kursangebot in der Fitnessbranche noch wenig abgedeckt. Workshops und Seminare mit Inhalten wie Analyse der eigenen Arbeitstechnik, Kennenlernen von Zeitfallen, Erfahrungsaustausch und Tipps zum Zeitmanagement sind deshalb sicher ein Bedürfnis. Folgenden Fragen müsste man in solchen Kursen nachgehen: 
- Werden die andern (wichtigeren) Tätigkeiten zuerst erledigt und anschliessend die Zeit für Administration gebraucht oder wird man durch die Papierarbeit von den eigentlich wichtigeren Tätigkeiten abgehalten?

- Lässt sich Papierarbeit schlecht delegieren oder können Schweizer FitnesscenterManager schlecht delegieren?

- Ist es günstiger, wenn der Chef die Administration macht oder fallen in einem mittleren Fitnessbetrieb zuwenig administrative Tätigkeiten für die Anstellung einer Sekretärin an, sodass die Chefin es gleich selbst erledigt?

- Welchen Zeitfallen falle ich zum Opfer? Welche könnte ich umgehen?

\subsection{Zusammenfassung „Führungsverhalten“}

- Wichtigste Führungstätigkeiten für den Erfolg (aus Sicht der Centerleiter): Personal führen, planen/koordinieren, motivieren/bestärken, Akquisition/Marketing.

- Sozialkompetenz kommt in der Fitnessbranche vor Fachkompetenz.

- Am meisten Zeit braucht Administration/Papierarbeit, die nur 9.wichtigste Führungstätigkeit.

- Weiterbildungsangebote im Bereich Sozial- und Selbstkompetenz sowie Arbeitstechnik und Zeitmanagement wären nötig, werden jedoch branchenspezifisch noch kaum angeboten. 


\section{Ergebnisse des Bausteins „Erfolg“}

\subsection{Persönliche Erfolgsbeurteilung}

\begin{tabular}{|l|r|}
\hline Persönliche Erfolgsbeurteilung & In \% \\
\hline Mässig erfolgreich & 31 \\
\hline Erfolgreich & 61 \\
\hline Sehr erfolgreich & 8 \\
\hline
\end{tabular}

Tabelle 12-1 Persönliche Erfolgsbeurteilung

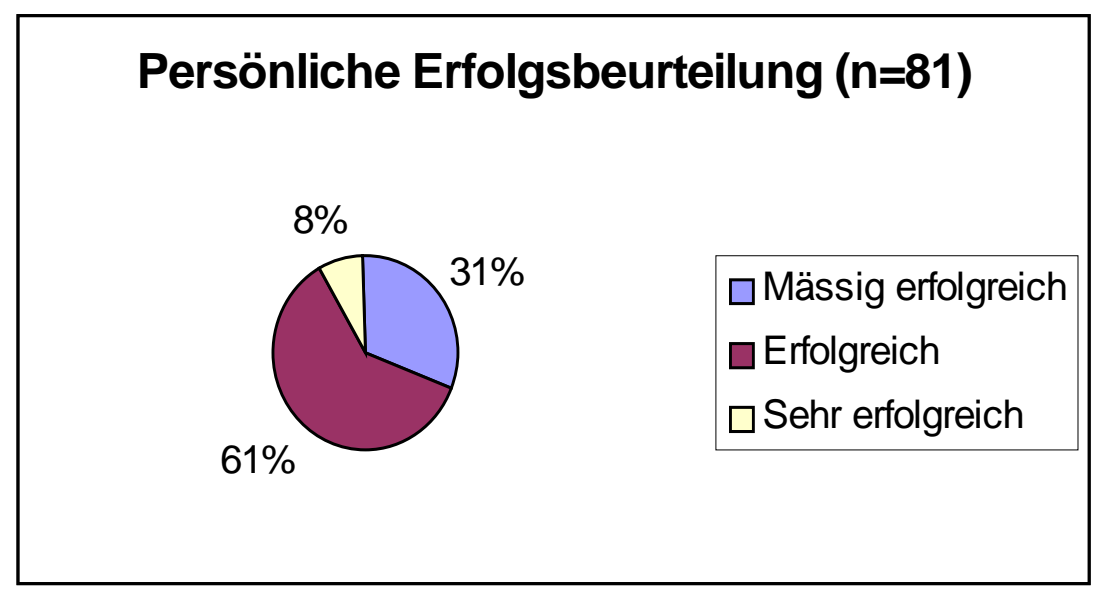

Abbildung 12-1 Persönliche Erfolgsbeurteilung

Grundsätzlich herrscht in der Fitnessbranche eine relative Zufriedenheit mit dem Geschäfts-gang. Nur ein Drittel aller Center bezeichnen sich als mässig erfolgreich, beinahe zwei Drittel bezeichnen ihr Geschäft als erfolgreich. Auffallend ist die kleine Spitze von nur gerade 8\%, die sich als sehr erfolgreich bezeichnen. Es wurden bei der Fragestellung nach der persönlichen Erfolgsbeurteilung keine Erfolgskriterien vorgegeben. Diese allgemeine Erfolgsbeurteilung ist also ein recht guter Gradmesser für die Grundzufriedenheit der Branche. Diese ist im Vergleich zu anderen Branchen sehr hoch: zwei Drittel aller Center bezeichnen sich als erfolgreich oder sehr erfolgreich.

Leider erhebt das Bundesamt für Statistik keine eigenen Zahlen nur für die Fitnesscenterbranche. Es kann somit nicht festgestellt werden, ob die grundsätzliche Zufriedenheit der Centerleiter mit einem guten Geschäftsgang im Vergleich mit anderen Branchen einhergeht. Immerhin konnte auf telefonische Nachfrage bei zwei Banken die Kreditrisiko-Einstufung von Fitnesscentern in Erfahrung gebracht werden. Bei beiden Banken gelten gutgeführte Fitnesscenter als grundsätzlich profitabel (5 - 8\% Gesamtkapitalrentabilität) und sind als „mässig risikoreich“ eingestuft.Die Einschätzung der Fitnesscenterleiter ist also im Vergleich mit der „objektiven“ Einschätzung der Banken verständlich und realistisch. 


\subsection{Centerfläche}

Die Verteilung der Center nach der Centerfläche wurde bereits in Tabelle 9-2 Flächenmässige Verteilung der Center vorgestellt. Um ein hin und her Blättern zu verhindern, ist sie hier nochmals aufgeführt.

\begin{tabular}{|l|r|r|}
\hline Fläche & Untersuchung & Gesamt-CH \\
\hline $0-200 \mathrm{~m}^{2}$ & $6 \%$ & $29 \%$ \\
\hline $0-300 \mathrm{~m}^{2}$ & $8 \%$ & $28 \%$ \\
\hline $301-560 \mathrm{~m}^{2}$ & $33 \%$ & $36 \%$ \\
\hline$>560 \mathrm{~m}^{2}$ & $53 \%$ & $8 \%$ \\
\hline
\end{tabular}

Gesamtzahlen CH von QUALITOP, 1999.

\section{Tabelle 12-2 Verteilung der CH-Center nach Fläche}

Mehr als die Hälfte der von Qualitop zertifizierten Center ist unter $300 \mathrm{~m}^{2}$ gross. Die Schweiz ist also ein Land der kleinen Center, was auch der Vergleich mit Deutschland klar zeigt (aus DSSV 1998). Dort sind 14\% der Center unter $400 \mathrm{~m}^{2}, 29 \%$ der Center zwischen $400 \mathrm{~m}^{2}$ und $600 \mathrm{~m}^{2}$ und $57 \%$ aller Center über 600. Leider sind in den beiden Ländern die Kategorien in der Erfassung anders gewählt, weshalb sich die Zahlen nicht genau vergleichen lassen. Die Tatsache, dass in Deutschland eher grössere Center vorkommen, ist aber klar ersichtlich.

In der Schweiz zeigen Neueröffnungen auch eher einen Trend in Richtung von grösseren Einheiten auf, dem auch einige kleine Center zum Opfer fallen werden (vergleiche 12.3 Mitgliederzahlen, Seite 84t. Es ist aber noch zu früh, um von einem eigentlichen Konzentrationsprozess in der Branche zu reden.

Immerhin, auch URSPRUNG (1998) weist in seiner Studie über das Berufsfeld Fitnesscenter auf Veränderungen und Gefahren hin:

Durch die hohe Dichte von Centern vor allem in Ballungszentren findet jedoch ein intensiver Preiskampf statt. Gefährdet sind vor allem mittelgrosse Center, die ihren Mitgliedern die ganze Angebotspalette zur Verfügung stellen wollen. Der Aufwand hierfür ist fast derselbe wie für ein sehr grosses Center oder eine Kette mit mehreren Betrieben, die Kosten zu amortisieren, ist jedoch viel schwieriger.

Die kleineren Center können nur dann überleben, wenn sie es schaffen, eine attraktive Nische im Angebot zu besetzen. Zudem haben sie den Vorteil, dass die Mitgliederbindung dank der grösseren Überschaubarkeit in der Regel besser ist. 


\subsection{Mitgliederzahlen}

Die folgenden Zahlen wurden in der Untersuchung ermittelt. Leider gibt es keine statistische Erfassung der Jahresabonnements der QUALITOP-Mitglieder. Es kann also kein Quervergleich der in dieser Untersuchung ermittelten Werte im gesamtschweizerischen Vergleich angestellt werden.

\begin{tabular}{|l|r|r|r|r|r|r|}
\hline & $\mathbf{1 9 9 5}$ & $\mathbf{1 9 9 6}$ & $\mathbf{1 9 9 7}$ & $\mathbf{1 9 9 5}$ & $\mathbf{1 9 9 6}$ & $\mathbf{1 9 9 7}$ \\
\hline $0-500$ & $30 \%$ & $27 \%$ & $31 \%$ & $56 \%$ & $43 \%$ & $42 \%$ \\
\hline $501-1000$ & $10 \%$ & $17 \%$ & $22 \%$ & $18 \%$ & $27 \%$ & $30 \%$ \\
\hline $1001-1500$ & $8 \%$ & $12 \%$ & $10 \%$ & $14 \%$ & $20 \%$ & $13 \%$ \\
\hline $1501-2000$ & $4 \%$ & $3 \%$ & $7 \%$ & $7 \%$ & $4 \%$ & $10 \%$ \\
\hline $2001-2500$ & $0 \%$ & $1 \%$ & $0 \%$ & $0 \%$ & $2 \%$ & $0 \%$ \\
\hline$>2500$ & $2 \%$ & $2 \%$ & $4 \%$ & $5 \%$ & $4 \%$ & $5 \%$ \\
\hline Keine Angaben & $46 \%$ & $38 \%$ & $26 \%$ & & & \\
\hline
\end{tabular}

Tabelle 12-3 Abonnementsverkäufe

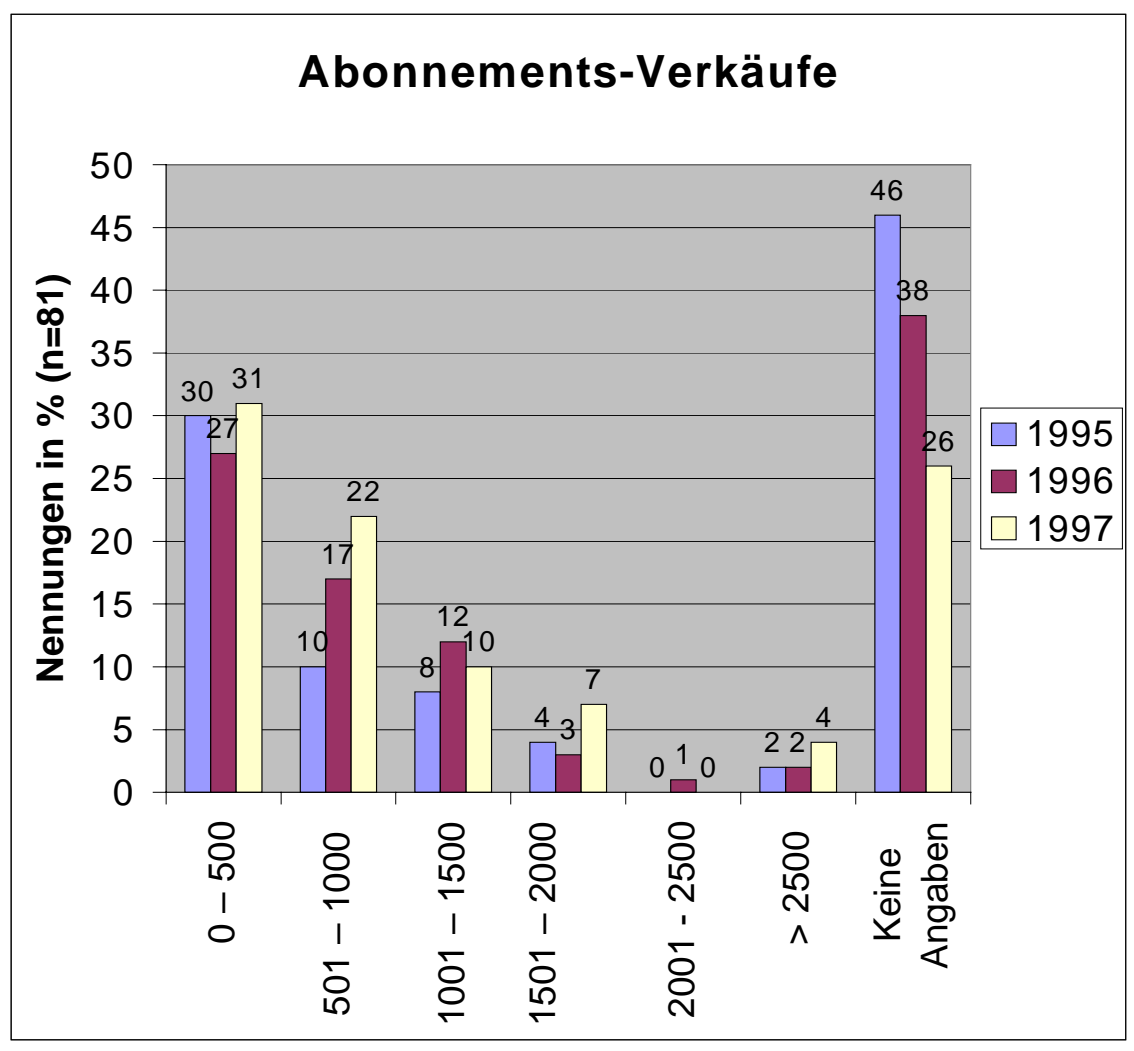

Abbildung 12-2 Abonnements-Verkäufe 
Es fallen verschiedene Dinge auf:

Viele Leerstimmen

Ein sehr hoher Prozentsatz an Centern hat keine Abonnementszahlen angegeben. In den Jahren 1995 und 1996 sind folgende Erklärungen möglich:

- Die 95er-Zahlen sind irgendwo im Keller gelagert und wurden für diese Untersuchung nicht hervorgeholt.

- Viele Center sind erst seit 2 Jahren im Geschäft.

- Die Geschäftsführerin hat in den letzten zwei Jahren gewechselt und hat die alten Zahlen nicht mehr.

Für das Jahr 1997 sind die Zahlen sicher bekannt. Immerhin 26\% der Befragten oder jeder vierte wollten diese Zahlen nicht veröffentlichen. Die Zahl der verkauften Abonnements scheint also bereits eine sensible Zahl $\mathrm{zu}$ sein, die man nicht gerne preisgibt.

Vor allem kleinere Center mit unter 1000 Abos

Etwa 70\% der Center, die eine Abo-Angabe gemacht haben, verkaufen unter 1000 Jahresabonnements. Dies stimmt überein mit den oben gemachten Aussagen zur Betriebsfläche. Die Schweiz ist eher ein Land der kleinen Center.

Verschiebungen bei den beiden untersten Segmenten

Die auffallendsten Verschiebungen betreffen die beiden untersten Segmente. Die Gruppe mit unter 500 Mitgliedern ist kontinuierlich von $56 \%$ auf $42 \%$ geschrumpft, die Gruppe mit 500 - 1000 Abonnements im Gegenzug kontinuierlich von 18\% auf 30\% angewachsen.

Die Gruppen 1000 - 2500 Mitglieder haben sich uneinheitlich entwickelt.Grundsätzlich ist ein Trend zu eher grösseren Centern feststellbar. Es gab unter den Neueröffnungen der letzten 2 Jahre in der Schweiz überproportional viele Center mit einer Fläche von über $1000 \mathrm{~m}^{2}$ und einer Abonnementszahl von über 2000 Abonnements.

\subsection{Einzeleintritte}

Nur gerade 10 der befragten 81 Center bieten Einzeleintritte an. Aus diesem Grund wird auf Resultate dieser Erhebung verzichtet.

\subsection{Entwicklung der Rendite}

Leider kann zur Renditeentwicklung keine Aussage gemacht werden. Nur gerade etwa $10 \%$ der befragten Center gaben ihre Rendite-Entwicklung an, auch wenn nur eine prozentuale Entwicklung ohne konkrete Zahlen gefragt wurde.

Es zeigt sich, dass in der Branche ein starker Konkurrenzdruck herrscht und man sich scheut, Zahlen zu veröffentlichen, die auch nur am Rande einem eventuellen Konkurrenten etwas nützen könnten. 
Ein Gespräch mit den Präsidenten des Schweizerischen Fitnesscenterverbandes SFCV bestätigte diese Zurückhaltung bei der Veröffentlichung von Zahlen. Trotz wiederholter Anstrengungen und Vorstösse sei es dem SFCV bis jetzt noch nicht möglich, die genauen Mitglieder- und Rentabilitätszahlen der eigenen Mitglieder-Center zu erheben. Als Richtzahl gab er eine Gesamtkapitalrentabilität von 4-5\% an.

\subsection{Investitionen während der letzten drei Jahre}

\begin{tabular}{|l|r|}
\hline Umfang der Investitionen & In \% \\
\hline Klein & 3 \\
\hline Mittel & 43 \\
\hline Gross & 43 \\
\hline Sehr gross & 11 \\
\hline
\end{tabular}

Tabelle 12-4 Umfang der getätigten Investitionen

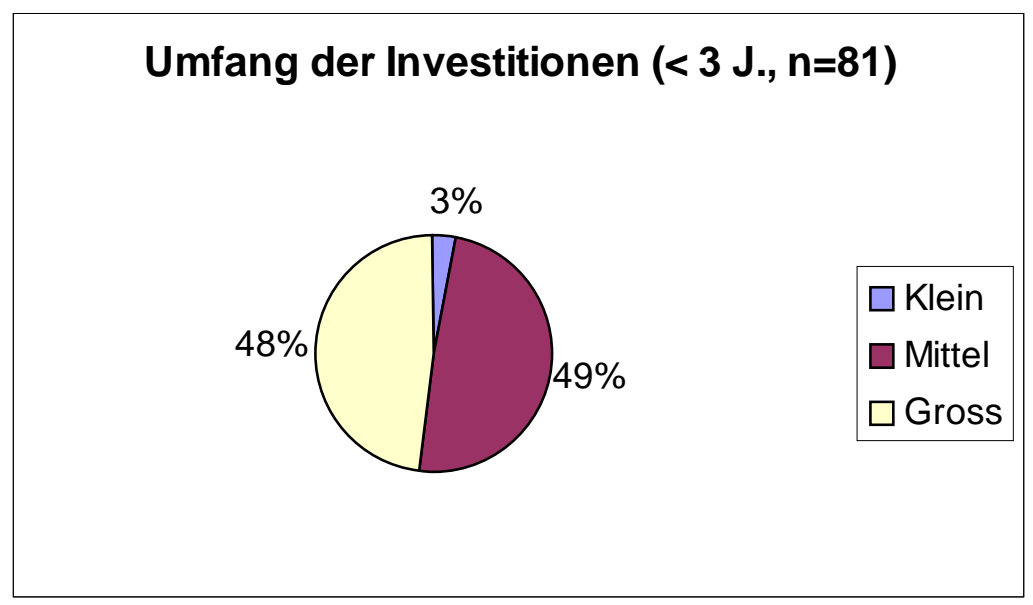

\section{Abbildung 12-3 Umfang der Investitionen der letzten drei Jahre}

Nur gerade $3 \%$ erklärten, sie hätten in den letzten drei Jahren wenig investiert. Die restlichen $97 \%$ gaben an, mittlere, grosse oder sogar sehr grosse Investitionen getätigt zu haben.

Offenbar herrscht in der Branche ein grosser Druck, auf dem neuesten Stand zu sein. Dies, obwohl „Moderne Maschinen“ erst an achter Stelle auf der Kundenwichtigkeitsliste steht (vergleiche Kapitel 13.2 Erfolgsfaktoren aus Kundensicht, Seite 101. 


\subsection{Detaillierte Erfolgsbeurteilung}

Folgende detaillierte Erfolgsbeurteilungen wurden abgegeben:

\begin{tabular}{|l|l|l|l|l|}
\hline Bereich & $\begin{array}{l}\text { Nicht } \\
\text { erfolgreich }\end{array}$ & $\begin{array}{l}\text { Mässig } \\
\text { erfolgreich }\end{array}$ & Erfolgreich & $\begin{array}{l}\text { Sehr } \\
\text { erfolgreich }\end{array}$ \\
\hline Mitgliederzahlen & $4 \%$ & $20 \%$ & $60 \%$ & $16 \%$ \\
\hline Auslastung Tag-Abend & $6 \%$ & $29 \%$ & $52 \%$ & $13 \%$ \\
\hline Auslastung saisonal & $6 \%$ & $54 \%$ & $37 \%$ & $3 \%$ \\
\hline Kundenzufriedenheit & $1 \%$ & $3 \%$ & $49 \%$ & $47 \%$ \\
\hline Gewinn & $12 \%$ & $49 \%$ & $35 \%$ & $5 \%$ \\
\hline Rentabilität & $11 \%$ & $47 \%$ & $36 \%$ & $6 \%$ \\
\hline & & & & \\
\hline
\end{tabular}

Tabelle 12-5 Persönliche Erfolgsbeurteilung nach Bereichen

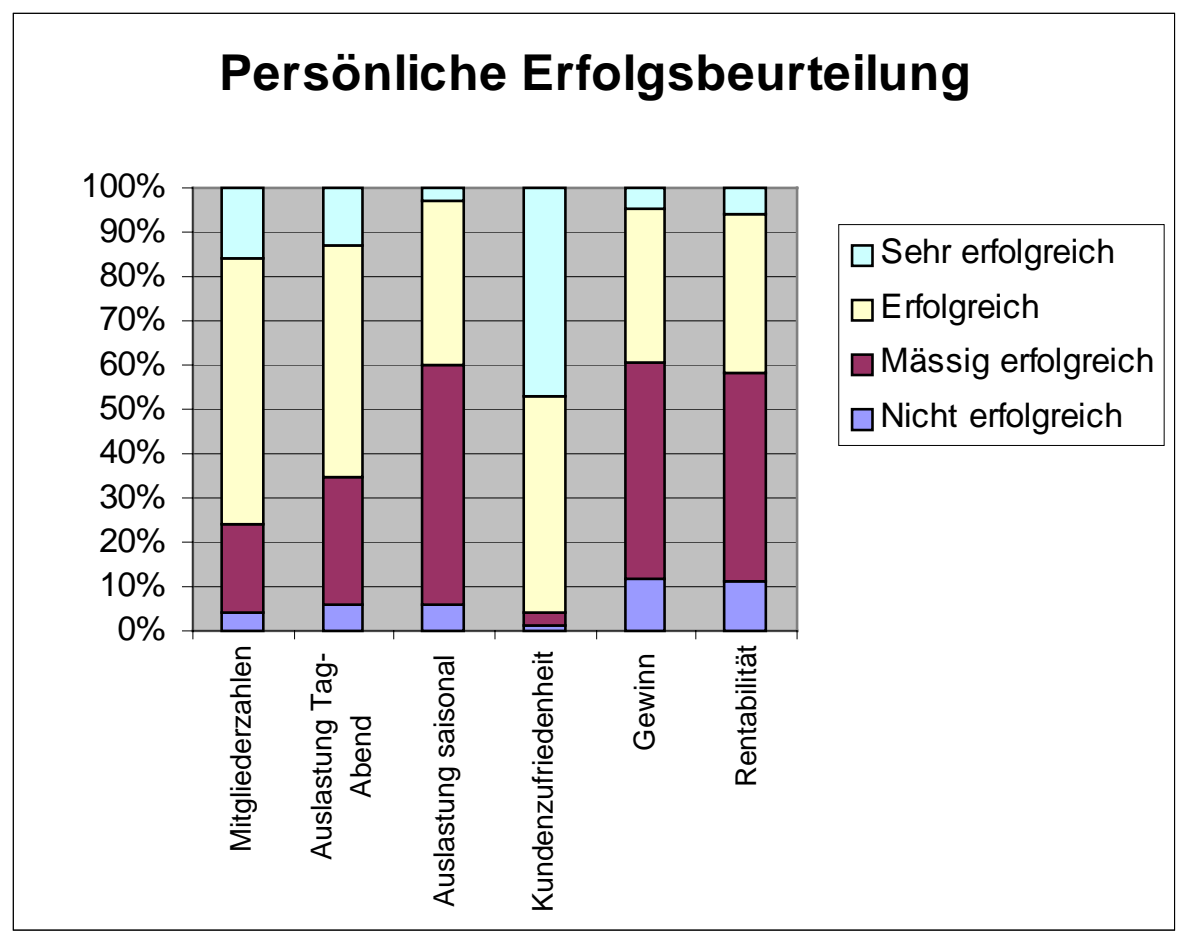

Abbildung 12-4 Persönliche Erfolgsbeurteilung

Am erfolgreichsten beurteilen sich die Managerinnen im Bereich der Kundenzufriedenheit. Knapp die Hälfte aller Center beurteilt sich als sehr erfolgreich im Bereich der Kundenzufriedenheit. Nur gerade $4 \%$ sehen sich als nicht oder mässig erfolgreich in diesem Bereich. 
Im Bereich der Mitgliederzahlen herrscht ebenfalls eine grosse Zufriedenheit. Drei Viertel der Manager bezeichnen ihren Geschäftsgang im Bereich Mitgliederzahlen als erfolgreich oder sehr erfolgreich.

Die Auslastungsverteilung Tag-Abend scheint in zwei Dritteln aller Center gut gemanagt zu sein. In der saisonalen Auslastungsverteilung ist man jedoch weniger zufrieden: $60 \%$ beurteilen sich in diesem Bereich als nicht oder mässig erfolgreich.

Erstaunlich sind nun die Resultate im Bereich von Gewinn und Rentabilität.

Trotz sehr grosser Kundenzufriedenheit und einer erfolgreich bis sehr erfolgreichen Mitgliederzahl wird der finanzielle Geschäftsgang im Bereich Gewinn und Rentabilität von jeweils etwa $60 \%$ der Center-Manager als nicht oder nur mässig erfolgreich beurteilt. Dies steht im Widerspruch zu den unter 12.1 Persönliche Erfolgsbeurteilung (Seite 82 gemachten Aussagen, dass es der Branche grundsätzlich nicht so schlecht geht. Die folgende Aufstellung zeigt, welche Betriebsgrösse am profitabelsten ist:

Sehr erfolgreich in Gewinn und Rentabilität:

$0-500$ Mitglieder: $\quad 1$ Center

1001 - 1500 Mitglieder: 3 Center

Erfolgreich in Gewinn und Rentabilität

0-500 Mitglieder: 8 Center

501 - 1000 Mitglieder: 6 Center

1001 - 1500 Mitglieder: 1 Center

> 2500 Mitglieder: 1 Center

Tabelle 12-6 Rentable Center nach Aboverkäufen

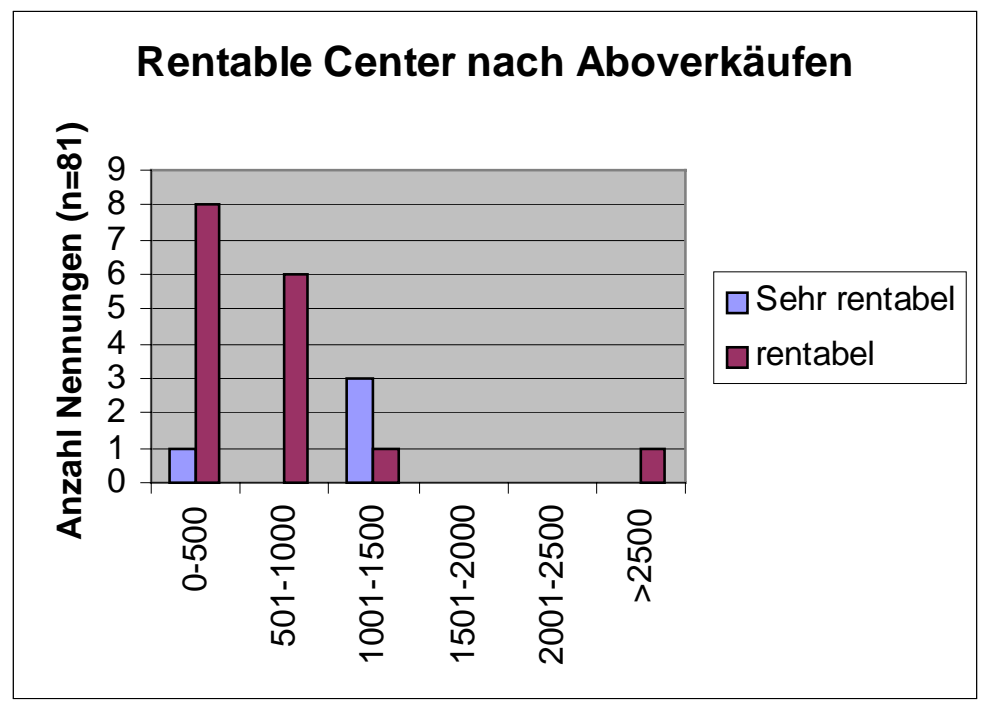

Abbildung 12-5 Rentable Center nach Abonnementsverkäufen 
Eine auffallend grosse Zahl an kleinen Centern mit $0-1000$ Mitgliedern scheint profitabel oder sehr profitabel zu sein. „Small is beautiful and profitable!“ könnte man versucht sein, zu sagen. An dieser Stelle sei auch nochmals an das Zitat von Ursprung (1998) erinnert (vergleiche 12.2 Centerfläche Seite 83, der auf die gute Mitgliederbindung der kleineren Center hinweist, was sich offensichtlich mit den Ergebnissen dieser Untersuchung deckt.

Eine weitere mögliche Erklärung für den schlechten Geschäftsgang der grösseren Center trotz zufriedener und vieler Kunden könnte sein, dass man im Winter wahrscheinlich sehr gut ausgelastet ist und kaum mehr Mitglieder verkraften könnte, im Sommer aber noch Lücken hat (vergleiche Abschnitt auf der vorangehenden Seite, Sparte saisonale Auslastung). Aufgrund der vorliegenden Informationen dieser Untersuchung können keine weiteren Erklärungen gegeben werden. Dies müsste in spezifischeren Befragungen ermittelt werden. Es lässt sich einzig feststellen, dass die Branche hier ein Problem hat und dass Handlungsbedarf besteht. 


\subsection{Persönliche Erklärungen für den Erfolg}

Folgende Erklärungen werden für den Erfolg im Bereich Mitgliederzahlen gegeben:

\begin{tabular}{|l|l|l|l|l|}
\hline Erklärung & $\begin{array}{l}\text { Kleiner } \\
\text { Einfluss }\end{array}$ & $\begin{array}{l}\text { Mittlerer } \\
\text { Einfluss }\end{array}$ & $\begin{array}{l}\text { Grosser } \\
\text { Einfluss }\end{array}$ & $\begin{array}{l}\text { Sehr gros- } \\
\text { ser Einfluss }\end{array}$ \\
\hline Marketing, Werbung & $14 \%$ & $34 \%$ & $28 \%$ & $24 \%$ \\
\hline $\begin{array}{l}\text { Marktforschung, Eingehen auf } \\
\text { Kundenwünsche }\end{array}$ & $10 \%$ & $32 \%$ & $38 \%$ & $20 \%$ \\
\hline Mitmachen von Trends & $22 \%$ & $60 \%$ & $13 \%$ & $5 \%$ \\
\hline Qualität der Infrastruktur & $3 \%$ & $10 \%$ & $49 \%$ & $38 \%$ \\
\hline Qualität des Personals & $2 \%$ & $3 \%$ & $22 \%$ & $73 \%$ \\
\hline Motivation/Teamgeist Personal & $0 \%$ & $3 \%$ & $27 \%$ & $70 \%$ \\
\hline Kostenmanagement & $10 \%$ & $24 \%$ & $52 \%$ & $14 \%$ \\
\hline Administration & $16 \%$ & $55 \%$ & $24 \%$ & $5 \%$ \\
\hline
\end{tabular}

Tabelle 12-XV Gründe für den Erfolg bei den Mitgliederzahlen

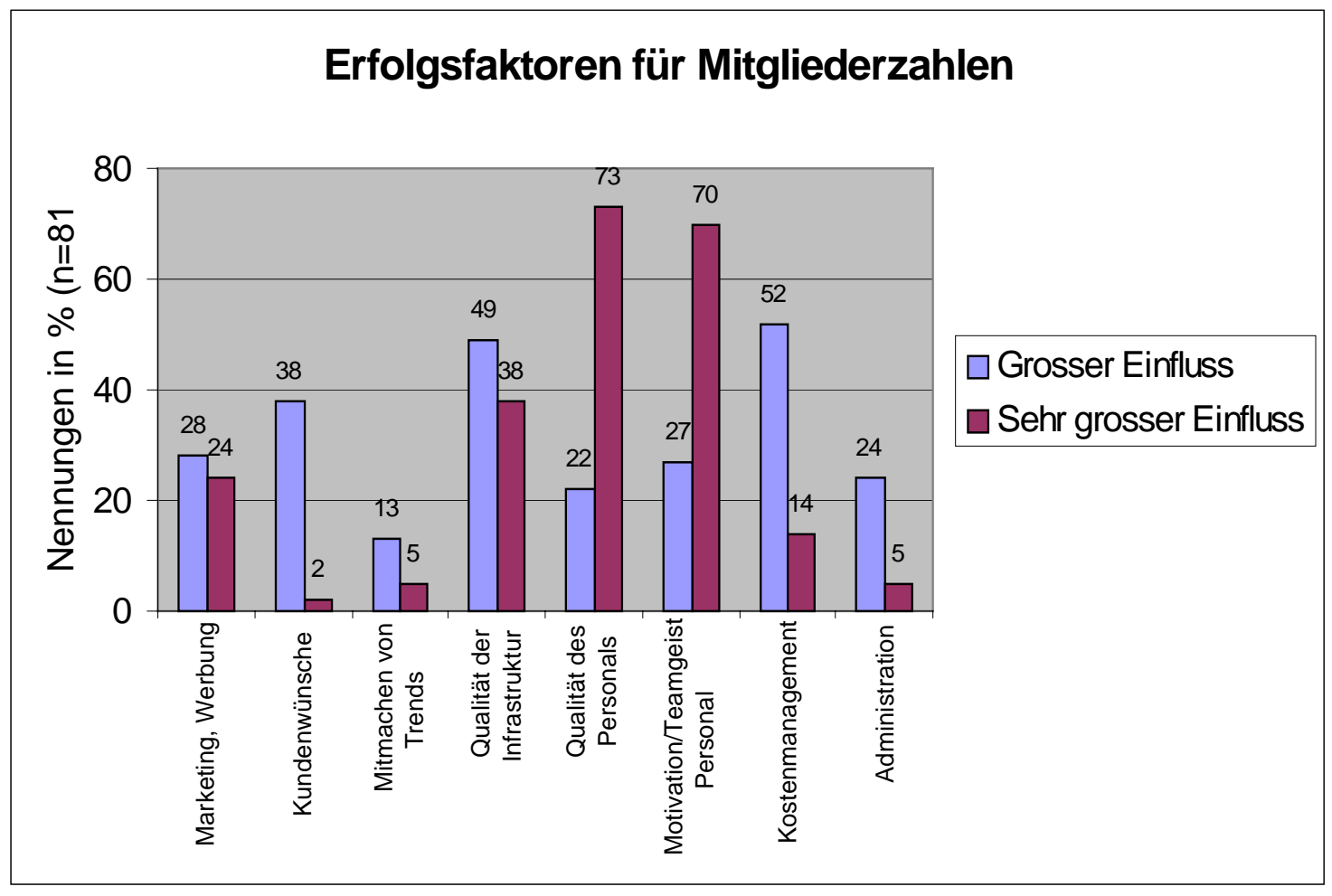

\section{Abbildung 12-6 Erfolgsfaktoren für Mitgliederzahlen}

Der mit grossem Abstand wichtigste Faktor für den Erfolg im Bereich Mitgliederzahlen ist das Personal. Sowohl die Qualität als auch die Motivation und der Teamgeist des 
Personals erhalten Höchstnoten. An zweiter Stelle kommt die Infrastruktur, noch vor Marketing oder Eingehen auf Kundenwünsche.

Folgende Erklärungen werden von den Managern gegeben, um ihren Erfolg im Bereich Auslastungsschwankungen zu begründen.

\begin{tabular}{|l|l|l|l|l|}
\hline Erklärung & $\begin{array}{l}\text { Kleiner } \\
\text { Einfluss }\end{array}$ & $\begin{array}{l}\text { Mittlerer } \\
\text { Einfluss }\end{array}$ & $\begin{array}{l}\text { Grosser } \\
\text { Einfluss }\end{array}$ & $\begin{array}{l}\text { Sehr gros- } \\
\text { ser Einfluss }\end{array}$ \\
\hline Marketing, Werbung & $20 \%$ & $44 \%$ & $27 \%$ & $9 \%$ \\
\hline $\begin{array}{l}\text { Marktforschung, Eingehen auf } \\
\text { Kundenwünsche }\end{array}$ & $16 \%$ & $32 \%$ & $42 \%$ & $10 \%$ \\
\hline Mitmachen von Trends & $42 \%$ & $44 \%$ & $9 \%$ & $5 \%$ \\
\hline Qualität der Infrastruktur & $24 \%$ & $21 \%$ & $40 \%$ & $15 \%$ \\
\hline Qualität des Personals & $13 \%$ & $14 \%$ & $33 \%$ & $40 \%$ \\
\hline Motivation/Teamgeist Personal & $13 \%$ & $15 \%$ & $32 \%$ & $40 \%$ \\
\hline Kostenmanagement & $33 \%$ & $33 \%$ & $27 \%$ & $7 \%$ \\
\hline Administration & $49 \%$ & $39 \%$ & $13 \%$ & $0 \%$ \\
\hline
\end{tabular}

Tabelle 12-XVI Gründe für den Erfolg bei Auslastungsschwankungen

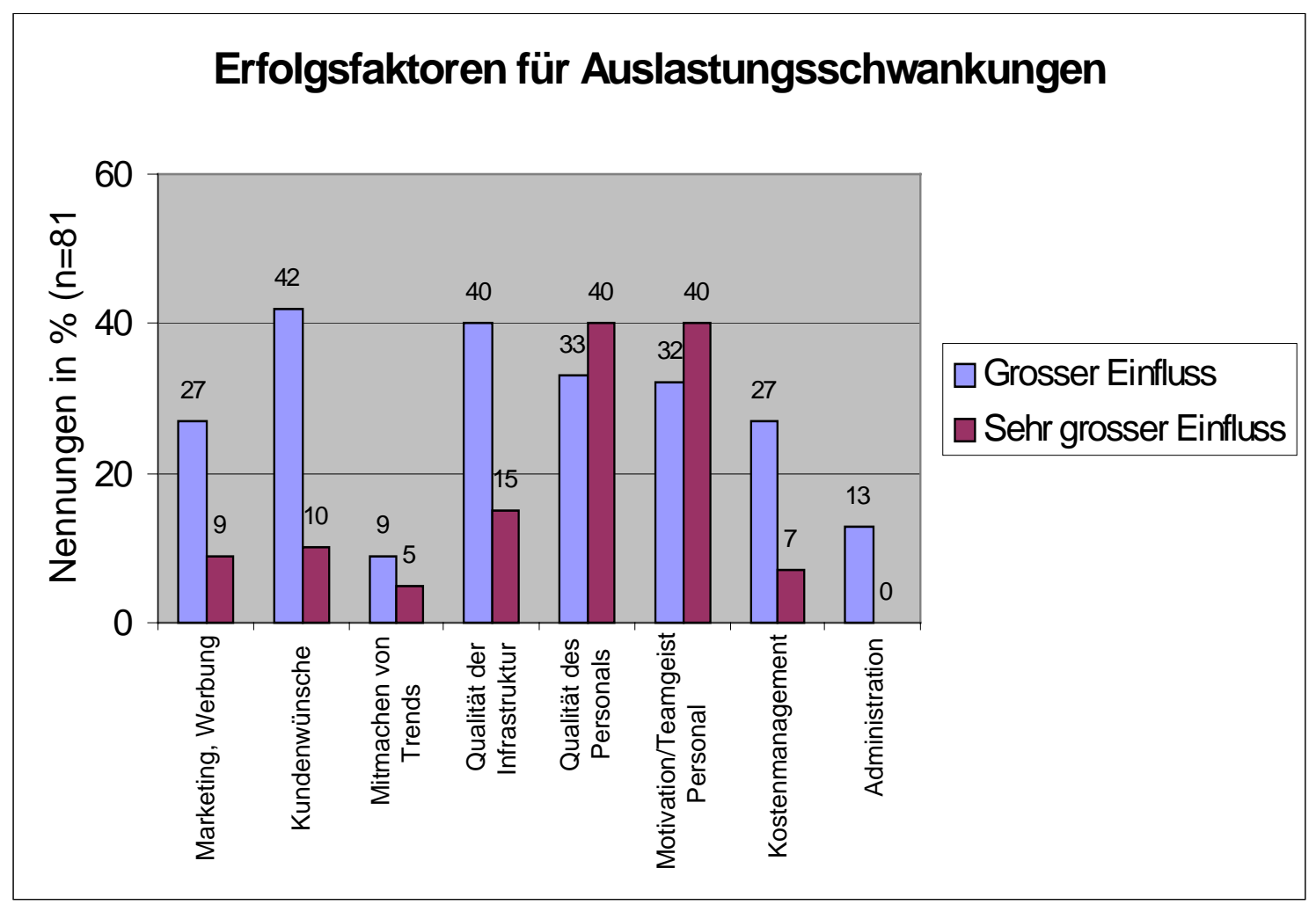

Abbildung 12-7 Erfolgsfaktoren für Auslastungsschwankungen 
Auch für den Erfolg bei den Auslastungsschwankungen werden wieder zum grössten Teil die Mitarbeiter verantwortlich gemacht. Auch hier kommen Qualität der Infrastruktur noch vor Marketing oder Eingehen auf Kundenwünsche. Dies ist eine sehr erstaunliche Aussage. Gerade Auslastungsschwankungen sind doch eigentlich ein Marketingproblem. Mit guten Anreizen für Randstunden, spezifischen Angeboten für Zielgruppen ausserhalb der Peak-Zeiten (Hausfrauen, Senioren, etc.) kann dem Problem griffig begegnet werden. Das Personal kann an zweiter Stelle auf die vorhandenen Angebote hinweisen und so mithelfen, die Auslastungsschwankungen zu vermindern. An erster Stelle muss aber ein entsprechendes Angebot und damit eine MarketingAnstrengung stehen.

Folgende Erklärungen werden von den Managern gegeben, um ihren Erfolg im Bereich Kundenzufriedenheit zu begründen.

\begin{tabular}{|l|l|l|l|l|}
\hline Erklärung & $\begin{array}{l}\text { Kleiner } \\
\text { Einfluss }\end{array}$ & $\begin{array}{l}\text { Mittlerer } \\
\text { Einfluss }\end{array}$ & $\begin{array}{l}\text { Grosser } \\
\text { Einfluss }\end{array}$ & $\begin{array}{l}\text { Sehr gros- } \\
\text { ser Einfluss }\end{array}$ \\
\hline Marketing, Werbung & $43 \%$ & $42 \%$ & $12 \%$ & $3 \%$ \\
\hline $\begin{array}{l}\text { Marktforschung, Eingehen auf } \\
\text { Kundenwünsche }\end{array}$ & $8 \%$ & $31 \%$ & $30 \%$ & $32 \%$ \\
\hline Mitmachen von Trends & $17 \%$ & $45 \%$ & $30 \%$ & $8 \%$ \\
\hline Qualität der Infrastruktur & $0 \%$ & $8 \%$ & $42 \%$ & $50 \%$ \\
\hline Qualität des Personals & $1 \%$ & $1 \%$ & $12 \%$ & $86 \%$ \\
\hline Motivation/Teamgeist Personal & $0 \%$ & $1 \%$ & $20 \%$ & $79 \%$ \\
\hline Kostenmanagement & $33 \%$ & $40 \%$ & $23 \%$ & $4 \%$ \\
\hline Administration & $25 \%$ & $49 \%$ & $25 \%$ & $1 \%$ \\
\hline
\end{tabular}

Tabelle 12-XVII Gründe für den Erfolg bei Kundenzufriedenheit 


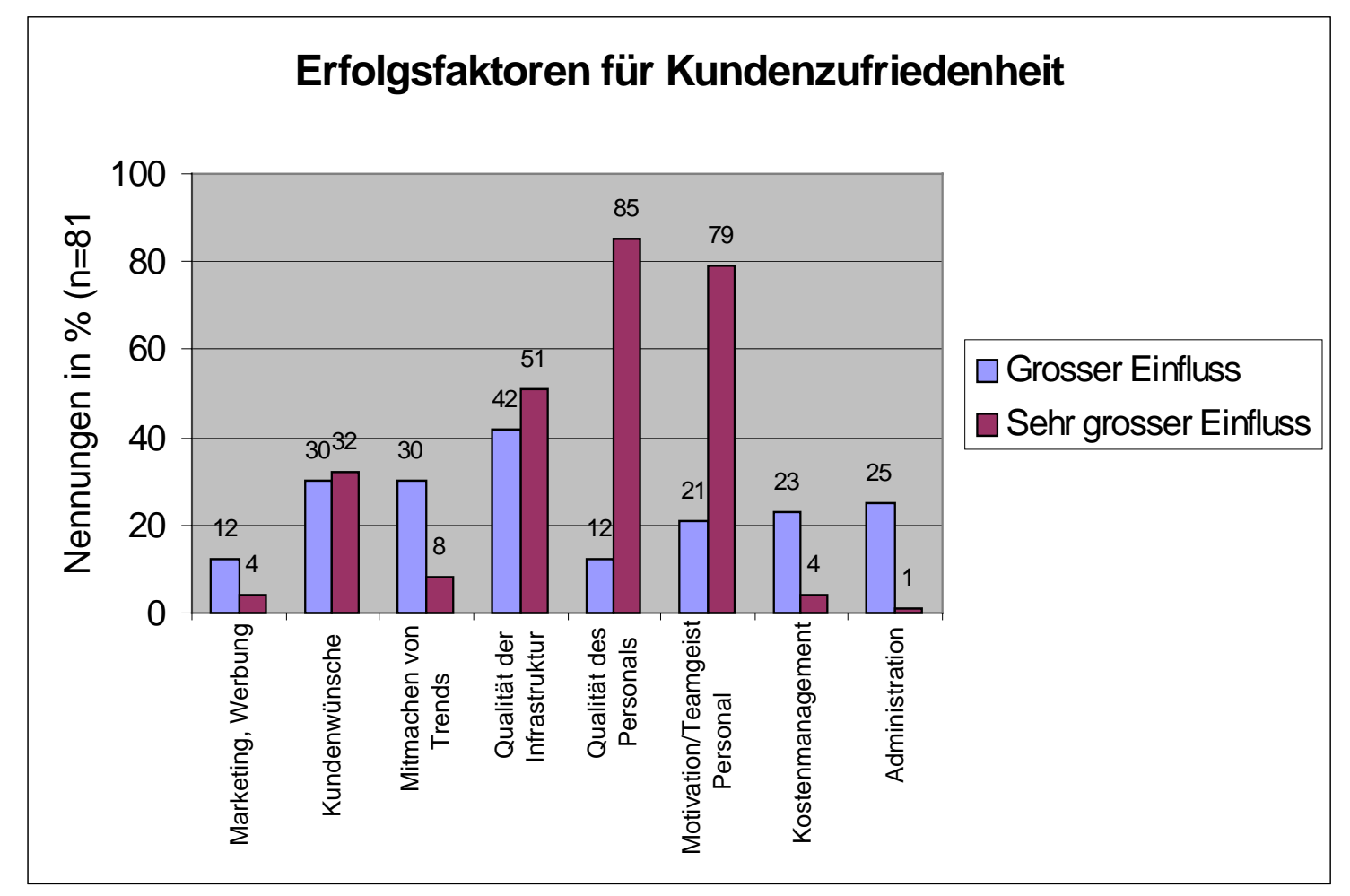

\section{Abbildung 12-8 Erfolgsfaktoren für Kundenzufriedenheit}

Qualität und Motivation des Personals sind die klaren Spitzenreiter. Beide Faktoren wurden von über drei Viertel aller Befragten als sehr wichtig eingestuft. An zweiter Stelle nach dem Personal wieder die Qualität der Infrastruktur, die von 50\% der Befragten als ,sehr wichtig“ für den Erfolg bei der Kundenzufriedenheit eingestuft wird.

Weit abgeschlagen ist der Faktor „Mitmachen von Trends“. Obwohl die Fitnessbranche sich in den letzten Jahren massiv entwickelt hat und immer wieder neue Trends aus den USA gekommen sind (Spinning, Body Pump, Step Aerobic, etc.), scheint bei den Fitnessanbietern die Überzeugung vorzuherrschen, dass übertriebenes Aufgreifen von Trends die Kundenzufriedenheit nicht massiv steigert.

In einem Quervergleich wurde gemessen, ob die Center, die sich als erfolgreich oder sehr erfolgreich bezeichnen, dem Mitmachen von Trends mehr oder weniger Gewicht zumessen als der Durchschnitt. Es konnte jedoch keine Signifikanz festgestellt werden. 
Folgende Erklärungen werden von den Managern gegeben, um ihren Erfolg im Bereich Gewinn zu begründen.

\begin{tabular}{|l|l|l|l|l|}
\hline Erklärung & $\begin{array}{l}\text { Kleiner } \\
\text { Einfluss }\end{array}$ & $\begin{array}{l}\text { Mittlerer } \\
\text { Einfluss }\end{array}$ & $\begin{array}{l}\text { Grosser } \\
\text { Einfluss }\end{array}$ & $\begin{array}{l}\text { Sehr gros- } \\
\text { ser Einfluss }\end{array}$ \\
\hline Marketing, Werbung & $17 \%$ & $26 \%$ & $27 \%$ & $30 \%$ \\
\hline $\begin{array}{l}\text { Marktforschung, Eingehen auf } \\
\text { Kundenwünsche }\end{array}$ & $14 \%$ & $33 \%$ & $30 \%$ & $23 \%$ \\
\hline Mitmachen von Trends & $26 \%$ & $49 \%$ & $17 \%$ & $8 \%$ \\
\hline Qualität der Infrastruktur & $3 \%$ & $22 \%$ & $42 \%$ & $33 \%$ \\
\hline Qualität des Personals & $1 \%$ & $8 \%$ & $27 \%$ & $64 \%$ \\
\hline Motivation/Teamgeist Personal & $3 \%$ & $9 \%$ & $30 \%$ & $58 \%$ \\
\hline Kostenmanagement & $7 \%$ & $17 \%$ & $35 \%$ & $41 \%$ \\
\hline Administration & $17 \%$ & $35 \%$ & $35 \%$ & $13 \%$ \\
\hline
\end{tabular}

Tabelle 12-XVIII Gründe für den Erfolg bei Gewinn

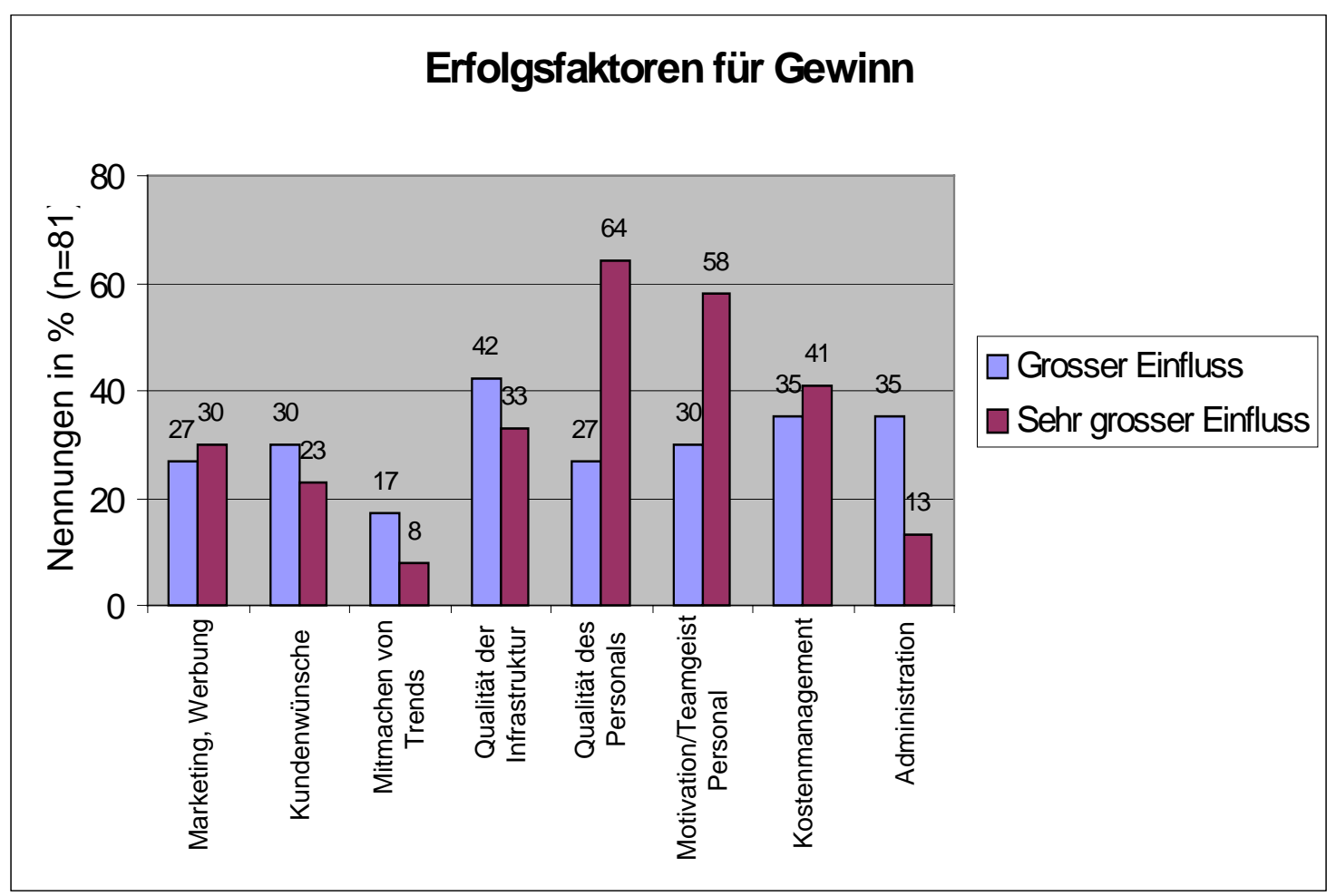

\section{Abbildung 12-9 Erfolgsfaktoren für Gewinn}

Hier taucht ein neuer Faktor unter den ersten drei auf: Kostenmanagement. Erstaunlicherweise kommt er aber erst auf Platz drei nach den beiden Personalfaktoren. Eine Mehrheit der Centerleiter sieht also das Personal auch im Bereich „Gewinn“ als wichtigsten Erfolgsfaktoren überhaupt an. 
Zusammenfassend lassen sich diese kritische Erfolgsfaktoren für die Teilbereiche bestimmen:

Mitgliederzahlen

1. Qualität des Personals

2. Motivation/Teamgeist des Personals

3. Qualität der Infrastruktur

4. Marktforschung, Eingehen auf Kundenwünsche

Auslastungsschwankungen

1. Qualität des Personals

2. Motivation/Teamgeist des Personals

3. Qualität der Infrastruktur

4. Marktforschung, Eingehen auf Kundenwünsche

Kundenzufriedenheit

1. Qualität des Personals

2. Motivation/Teamgeist des Personals

3. Qualität der Infrastruktur

4. Marktforschung, Eingehen auf Kundenwünsche

Gewinn

1. Qualität des Personals

2. Motivation/Teamgeist des Personals

3. Kostenmanagement

4. Qualität der Infrastruktur

Tabelle 12-XIX Kritische Erfolgsfaktoren für den Unternehmenserfolg

\subsection{Erfolgsfaktor Effizienz}

Folgende drei Effizienzkennzahlen lassen sich aufgrund der erhobenen Daten berechnen: Fläche pro Mitarbeiter, Fläche pro Mitglied, Mitglied pro Mitarbeiter

\section{Fläche pro Mitarbeiter}

\begin{tabular}{|l|r|}
\hline Fläche/Mitarbeiter & Verteilung \\
\hline $0-100 \mathrm{~m}^{2}$ & $23 \%$ \\
\hline $100-200 \mathrm{~m}^{2}$ & $50 \%$ \\
\hline $200-300 \mathrm{~m}^{2}$ & $21 \%$ \\
\hline$>300 \mathrm{~m}^{2}$ & $6 \%$ \\
\hline Durchschnittswert/Kennzahl & $\mathbf{1 6 0 ~ ~ m ^ { 2 }} / \mathbf{M A}$ \\
\hline
\end{tabular}

Tabelle 12-7 Effizienz: Fläche/Mitarbeiter 
$50 \%$ aller Center weisen eine Fläche von $100-200 \mathrm{~m}^{2}$ pro Mitarbeiter aus. Als Durchschnittswert der 81 untersuchten Center und somit quasi als Branchenkennzahl konnten $160 \mathrm{~m}^{2}$ pro Mitarbeiter berechnet werden.

Vergleicht man diese Kennzahl bei den sehr erfolgreichen und den nur mässig erfolgreichen Centern, so ergibt sich folgendes Bild:

\begin{tabular}{|l|r|}
\hline Center & Kennzahl m $/$ MA \\
\hline Sehr erfolgreiche Center & $168 \mathrm{~m}^{2} / \mathrm{MA}$ \\
\hline Wenig erfolgreiche Center & $125 \mathrm{~m}^{2} / \mathrm{MA}$ \\
\hline Durchschnitt aller Center & $160 \mathrm{~m}^{2} / \mathrm{MA}$ \\
\hline
\end{tabular}

\section{Tabelle 12-8 Vergleich Fläche/Mitarbeiter}

Es zeigt sich, dass erfolgreiche Center weniger Mitarbeiter für die gleiche Fläche einsetzen als die weniger erfolgreichen. Die eingesparten Personalkosten wirken sich offensichtlich direkt auf den Erfolg aus.

\section{Fläche pro Mitglied}

\begin{tabular}{|l|r|}
\hline Fläche/Mitglied & Verteilung \\
\hline $0-1 \mathrm{~m}^{2}$ & $37 \%$ \\
\hline $1-2 \mathrm{~m}^{2}$ & $33 \%$ \\
\hline $2-3 \mathrm{~m}^{2}$ & $22 \%$ \\
\hline$>3 \mathrm{~m}^{2}$ & $8 \%$ \\
\hline & \\
\hline Durchschnittswert/Kennzahl & $\mathbf{1 . 9} \mathbf{~ m}^{\mathbf{2}} / \mathbf{M g l}$. \\
\hline
\end{tabular}

Tabelle 12-9 Effizienz: Fläche/Mitglied

$37 \%$ aller Center weisen eine Fläche von $0-1 \mathrm{~m}^{2}$ pro Mitarbeiter, $33 \%$ eine Fläche von $1-2 \mathrm{~m}^{2}$ pro Mitarbeiter aus. Als Durchschnittswert der 81 untersuchten Center und somit quasi als Branchenkennzahl konnten $1.9 \mathrm{~m}^{2}$ pro Mitarbeiter berechnet werden.

Als Vergleich können hier die Zahlen von Deutschland (DSSV 1998) angefügt werden. Die ca. 5500 kommerziellen Fitnessanlagen in Deutschland kommen auf eine durchschnittliche Anzahl Mitglieder von 620 und haben eine durchschnittliche Fläche von $715 \mathrm{~m}^{2}$. Dies ergibt eine Fläche/Mitglied-Kennzahl von $1.2 \mathrm{~m}^{2}$. Diese Zahl ist deutlich kleiner als in der Schweiz. Es mag einerseits daran liegen, dass in Deutschland die Center grundsätzlich besser (effizienter) ausgelastet sind, andererseits ist in der Schweiz der Wellnes- und Sauna-Bereich eher grosszügiger gestaltet, was sofort recht viel Fläche beansprucht, die nicht so stark ausgelastet werden kann wie der Gerätebereich. 
Der Vergleich von sehr erfolgreichen Center und mässig erfolgreichen Centern im Bereich der Fläche pro Kunde präsentiert sich folgendermassen:

\begin{tabular}{|l|r|}
\hline Center & Kennzahl m² Mitglied \\
\hline Sehr erfolgreiche Center & $2.9 \mathrm{~m}^{2} /$ Mitglied \\
\hline Wenig erfolgreiche Center & $1.8 \mathrm{~m}^{2} /$ Mitglied \\
\hline Durchschnitt aller Center & $1.9 \mathrm{~m}^{2} /$ Mitglied \\
\hline
\end{tabular}

Tabelle 12-10 Vergleich Fläche/Mitglied

Man hätte erwartet, dass die erfolgreicheren Center pro Mitglied weniger Fläche zur Verfügung stellen oder anders gesagt, die vorhandene Fläche mit mehr Kunden besser nutzen. Erstaunlicherweise zeigt sich aber gerade das Gegenteil: die sehr erfolgreichen Center stellen dem Kunden über einen $\mathrm{m}^{2}$ mehr zur Verfügung als die nur mässig erfolgreichen Center.

Für die Kunden ist es also wahrscheinlich sehr wichtig, dass sie genügend Platz zur Verfügung haben. Leider wurde dieser Faktor ,genügend Platz“ in der Untersuchung „Wichtigkeit für den Kunden“ nicht erhoben. Der Faktor „helle, hohe Räumlichkeiten“ wurde erhoben, fungiert jedoch auf der Rangliste der Wichtigkeit für den Kunden erst an fünfter Stelle.

Es können mit den vorhandenen Daten keine weiteren Begründungen zu diesen Befunden gemacht werden. Dieser Frage sollte aber in weiteren Studien unbedingt nachgegangen werden.

Es zeigen sich hier gewisse Parallelen zur Hotellerie. Es ist auch dort so, dass erfolgreichere Hotels ihren Kunden mehr Platz zur Verfügung stellen, als weniger erfolgreiche (REHKUGLER 1997).

\section{Mitglieder pro Mitarbeiter}

\begin{tabular}{|l|r|}
\hline Mitglieder/Mitarbeiter & Verteilung \\
\hline $0-100$ & $38 \%$ \\
\hline $100-200$ & $40 \%$ \\
\hline $200-300$ & $20 \%$ \\
\hline$>300$ & $2 \%$ \\
\hline & \\
\hline Durchschnittswert/Kennzahl & 129 Mitgl. / MA \\
\hline
\end{tabular}

Tabelle 12-11 Effizienz: Mitglied/Mitarbeiter

$38 \%$ aller Center beschäftigen pro 0 - 100 Mitglieder einen Mitarbeiter, $40 \%$ pro $100-$ 200 Mitglieder einen Mitarbeiter. Als Durchschnittswert der 81 untersuchten Center und 
somit quasi als Branchenkennzahl konnten 129 Mitglieder pro Mitarbeiter berechnet werden.

Wieder wurden sehr erfolgreiche und mässig erfolgreiche Center gegenübergestellt:

\begin{tabular}{|l|r|}
\hline Center & Kennzahl Mitgl./Mitarbeiter \\
\hline Sehr erfolgreiche Center & 120 Mitglieder/Mitarbeiter \\
\hline Wenig erfolgreiche Center & 115 Mitglieder/Mitarbeiter \\
\hline Durchschnitt aller Center & 129 Mitglieder/Mitarbeiter \\
\hline
\end{tabular}

\section{Tabelle 12-12 Vergleich Mitglieder/Mitarbeiter}

Sowohl erfolgreiche als auch weniger erfolgreiche Center haben weniger Mitglieder pro Mitarbeiter als der Branchenschnitt, gewährleisten also eine quantitativ überdurchschnittliche Betreuung. Die Ergebnisse können dahingehend interpretiert werden, dass es nicht entscheidend ist, wieviel Personal da ist (Quantität), sondern wie die effektive Betreuungsarbeit geleistet wird (Qualität). Die Qualitätsdimension konnte aber in dieser Studie aus Ressourcengründen nicht miteinbezogen werden. Mit den vorhandenen Daten lässt sich die Vermutung also nicht bestätigen. Auch dies wäre eine sehr interessante Fragestellung für eine Fortsetzungsstudie.

\subsection{Zusammenfassung „Erfolg“}

- Die Fitnessbranche ist eine erfolgreiche Branche. Fast zwei Drittel aller Center beurteilen ihr Geschäft als erfolgreich oder sehr erfolgreich.

- Die Schweiz ist ein Land der kleinen Fitnesscenter $\left(<300 \mathrm{~m}^{2}\right)$. An der Untersuchung beteiligten sich jedoch vor allem grössere Center $\left(>300 \mathrm{~m}^{2}\right)$.

- Die ganz kleinen Center $(<500$ Mitglieder) nehmen tendenziell ab zu Lasten der mittleren Center (500 - 1000 Abos). Die grossen Center mit über 1000 Mitgliedern entwickeln sich uneinheitlich.

- Renditezahlen werden aus Konkurrenzgründen nicht bekanntgegeben. Es ist von einer Gesamtkapitalrentabilität um 5\% auszugehen.

- Es herrscht ein grosser Innovationsdruck in der Branche. Über 50\% der Center haben in den letzten 3 Jahren grosse oder sehr grosse Investitionen getätigt.

- Kritische Erfolgsfaktoren sind

- Qualität des Personals

- Freundlichkeit des Personals

- Qualität der Infrastruktur

- Eingehen auf Kundenwünsche/Marktforschung 
- Folgende Durchschnittswerte konnten als Kennzahlen ermittelt werden:

- Fläche/Mitarbeiter: $\quad 160 \mathrm{~m}^{2} /$ Mitarbeiter

- Fläche/Mitglied: $\quad 1.9 \mathrm{~m}^{2} /$ Mitglied

- Mitglieder/Mitarbeiter: 129 Mitglieder/Mitarbeiter

- Sehr erfolgreiche Fitnesscenter unterscheiden sich in den Kennzahlen von ihren mässig erfolgreichen Kollegen wie folgt:

- grössere Fläche pro Mitarbeiter

- grössere Fläche pro Mitglied

- gleich viele Mitglieder pro Mitarbeiter 


\section{Ergebnisse des Bausteins „Situation“}

\subsection{Konkurrenzdruck}

Der Konkurrenzdruck wird wie folgt beschrieben:

\begin{tabular}{|l|r|}
\hline Konkurrenzdruck & In \% \\
\hline Klein & 13 \\
\hline Erträglich & 43 \\
\hline Gross & 30 \\
\hline Sehr gross & 14 \\
\hline
\end{tabular}

Tabelle 13-1 Konkurrenzdruck

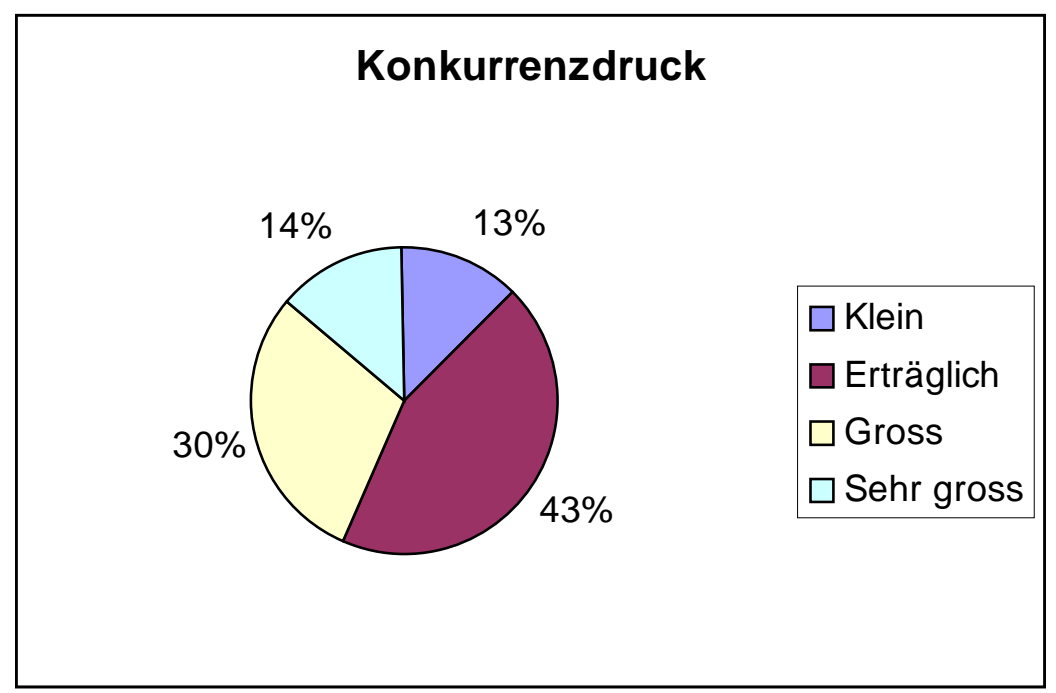

\section{Abbildung 13-1 Konkurrenzdruck}

Der Konkurrenzdruck wird von mehr als der Hälfte der Managerinnen als klein oder erträglich beurteilt. Dies steht im Gegensatz zu den Feststellungen im Unterkapitel 12 Ergebnisse des Bausteins „Erfolg " (Seite 82). Dort wurde bemerkt, dass die Manager aussergewöhnlich vorsichtig sind, wenn es um das Herausgeben von sogenannten sensiblen Daten wie Abo-Verkäufe, Rentabilität oder Gewinn geht. Daraus wurde geschlossen, dass der Konkurrenzdruck eher gross ist. In persönlichen Gesprächen wurde dies auch immer wieder bestätigt. Offensichtlich ziehen es aber viele vor, in dieser quasi offiziellen Umfrage im Bereich Konkurrenzdruck auf Understatement zu machen. 


\subsection{Erfolgsfaktoren aus Kundensicht}

Das erfolgreiche Anbieten einer Dienstleistung hängt im Wesentlichen davon ab, dass man genau weiss, wie der Kunde die Leistung beurteilt.

Im Beispiel des Fitnesscenters bestimmen verschiedene Faktoren die Dienstleistung. Es ist kaum möglich, alle Kriterien, die für den Kunden wichtig sind, zu erfüllen. Es sei denn, der Preis des Produkts spielt gar keine Rolle. Im Normalfall geht es also darum, möglichst viele der wichtigsten Kundenkriterien möglichst gut zu erfüllen. Die folgende Frage hatte die Absicht, zu klären, welche Kriterien für den Kunden wichtig sind. Die Liste der 12 Kriterien kam wie folgt zustande:

Brainstorming mit 10 Kunden aus verschiedenen Centern zur Frage: Was ist Ihnen bei der Auswahl Ihres Fitnesscenters besonders wichtig. Die etwa 30 Kriterien wurden zu Kategorien zusammengefasst und von einer Kontrollgruppe von 10 anderen Kunden nach der Wichtigkeit beurteilt. Die 12 wichtigsten Kriterien wiederum wurden den Fitnesscenter-Managern zur Beurteilung vorgelegt.

Auf die Frage „Wie wichtig sind die folgenden Faktoren für Ihre Kunden?“ wurden folgende Antworten erteilt:

\begin{tabular}{|l|l|l|l|l|}
\hline Faktor & Unwichtig & $\begin{array}{l}\text { Ziemlich } \\
\text { wichtig }\end{array}$ & Wichtig & $\begin{array}{l}\text { Sehr } \\
\text { wichtig }\end{array}$ \\
\hline Breite des Angebots & $9 \%$ & $14 \%$ & $48 \%$ & $29 \%$ \\
\hline Moderne Maschinen und Geräte & $2 \%$ & $27 \%$ & $47 \%$ & $24 \%$ \\
\hline Sauberkeit der Nasszone & $1 \%$ & $4 \%$ & $15 \%$ & $80 \%$ \\
\hline Kinderbetreuung & $25 \%$ & $24 \%$ & $31 \%$ & $20 \%$ \\
\hline Versch. Abo-Möglichkeiten & $6 \%$ & $25 \%$ & $38 \%$ & $31 \%$ \\
\hline Zentrale Lage & $12 \%$ & $20 \%$ & $44 \%$ & $24 \%$ \\
\hline Anzahl Parkplätze & $4 \%$ & $15 \%$ & $42 \%$ & $41 \%$ \\
\hline Erreichbarkeit mit öV & $15 \%$ & $37 \%$ & $40 \%$ & $8 \%$ \\
\hline Freundlichkeit des Personals & $0 \%$ & $1 \%$ & $10 \%$ & $89 \%$ \\
\hline Kompetenz des Personals & $0 \%$ & $3 \%$ & $21 \%$ & $76 \%$ \\
\hline Kollegen trainieren hier & $18 \%$ & $19 \%$ & $42 \%$ & $21 \%$ \\
\hline Helle, hohe Räumlichkeiten & $3 \%$ & $17 \%$ & $48 \%$ & $32 \%$ \\
\hline
\end{tabular}

Tabelle 13-2 Wichtigkeit von situativen Faktoren aus Kundensicht 


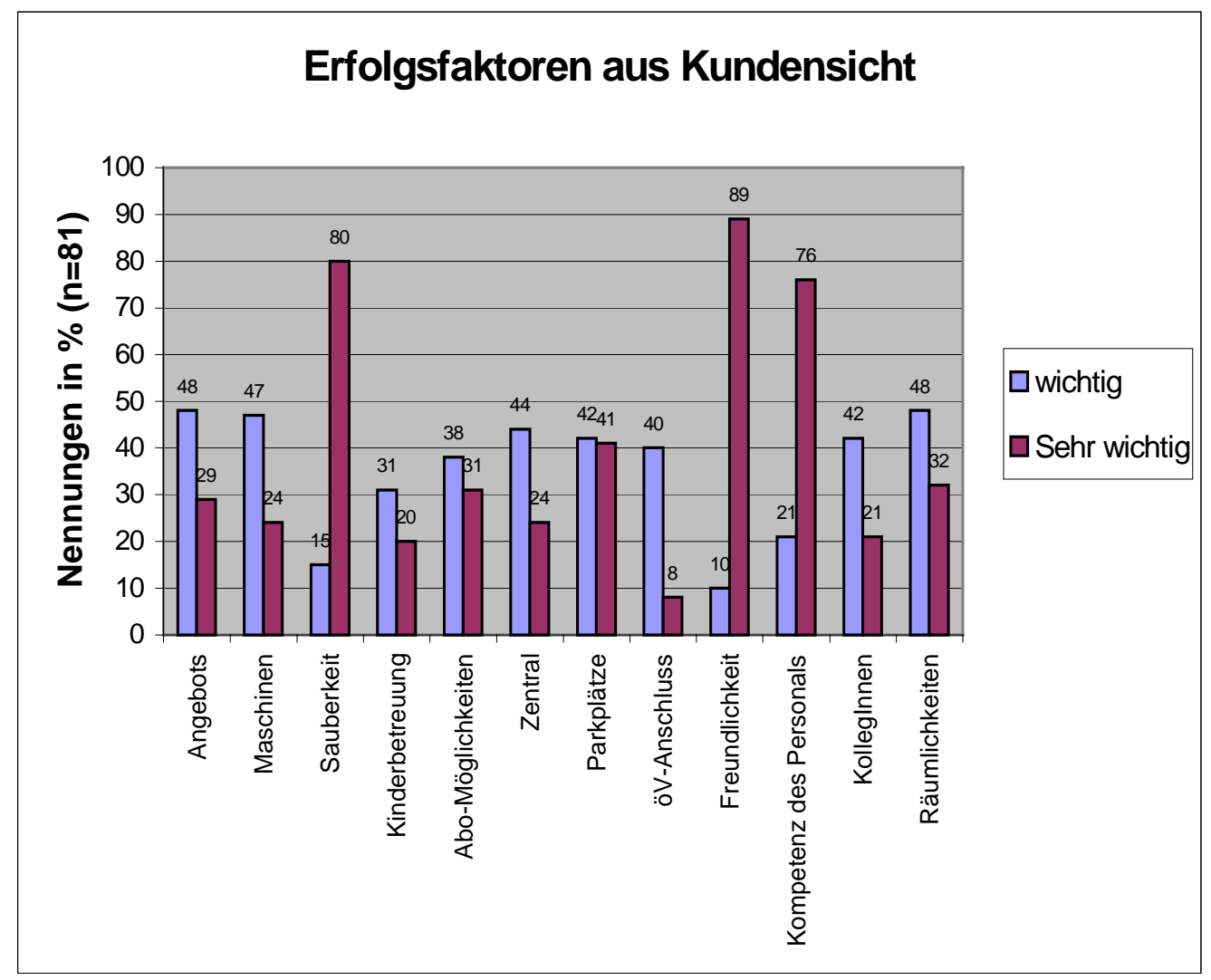

\section{Abbildung 13-2 Erfolgsfaktoren aus Kundensicht}

Dies ergibt folgende Hitliste

\begin{tabular}{|l|l|l|}
\hline Rang & Faktor & $\begin{array}{l}\text { Durchschnitts- } \\
\text { Rating }\end{array}$ \\
\hline 1 & Freundlichkeit des Personals & 3.88 \\
\hline 2 & Kompetenz des Personals & 3.74 \\
\hline 3 & Sauberkeit der Nasszone & 3.73 \\
\hline 4 & Anzahl Parkplätze & 3.16 \\
\hline 5 & Helle, hohe Räumlichkeiten & 3.10 \\
\hline 6 & Breite des Angebots & 2.99 \\
\hline 7 & Verschiedene Abonnements-Möglichkeiten & 2.94 \\
\hline 8 & Moderne Maschinen und Geräte & 2.91 \\
\hline 9 & Zentrale Lage & 2.79 \\
\hline 10 & Kollegen trainieren hier & 2.65 \\
\hline 11 & Kinderbetreuung & 2.46 \\
\hline 12 & Erreichbarkeit mit öffentlichen Verkehrsmitteln & 2.42 \\
\hline
\end{tabular}

Legende: Durchschnittsrating ( $\mathrm{n}=81)$ : 1 = unwichtig, $2=$ ziemlich wichtig, 3 = wichtig, $4=$ sehr wichtig.

Tabelle 13-3 Hitliste der Erfolgsfaktoren aus Kundensicht 
Die drei mit Abstand wichtigsten Erfolgsfaktoren aus Kundensicht sind nach der Meinung der 81 befragten Manager

- Freundlichkeit des Personals

- Kompetenz des Personals

- Sauberkeit der Nasszone

Dies deckt sich mit den Aussagen, die im Unterkapitel 12.8 Persönliche Erklärungen für den Erfolg (Seite 90) gemacht werden. Dort werden für die vier Erfolgskriterien „Mitgliederzahlen“, Auslastungsschwankungen“, Kundenzufriedenheit" und „Gewinn“ jeweils immer „Kompetenz des Personals“ und Freundlichkeit des Personals“ als wichtigste Ursachen genannt.

An zweiter Stelle folgen die beiden Faktoren „Anzahl Parkplätze“ und „Helle, hohe Räumlichkeiten“.

Erst an dritter Stelle - bereits mit fast einem Wichtigkeits-Wertungspunkt weniger folgt das Trio „Breite des Angebots“, Verschiedene Abonnements-Möglichkeiten“ und „Moderne Maschinen und Geräte“.

Wie bereits eingangs dieses Unterkapitels erwähnt, konzentriert sich effektives Management darauf, die für den Kunden wichtigsten Faktoren möglichst gut zu erfüllen. Bevor also an neue Maschinen, neue Abonnementsmöglichkeiten oder andere Preise gedacht wird, sind die Faktoren

- Freundlichkeit des Personals

- Kompetenz des Personals

- Sauberkeit der Nasszone

- Anzahl Parkplätze

- Helle, hohe Räumlichkeiten

zu optimieren. An den Parkplätzen oder an den Räumlichkeiten lässt sich meistens wenig verändern. Einzig bei einem Center-Neubau oder einer Übernahmen kann man auf diese Faktoren achten. Im Bereich Personalführung und Sauberkeit besteht jedoch nach Einschätzung der Manager noch einiges an Verbesserungspotential (vergleiche Unterkapitel 13.3 Erfüllung der Kundenanforderungen. Seite 104.

Neben den vorgegebenen Faktoren bestand die Möglichkeit, eigene Faktoren, die ebenfalls für den Kunden wichtig sind, zu nennen.

Folgende zusätzlichen Faktoren wurden genannt $(\mathrm{n}=81)$ :

- Betreuung durch das Personal: 18

- Atmosphäre: 11

- Preis: 8

- Öffnungszeiten: 7

- Physiotherapie/medizinische Betreuung im Haus: 6

- Krankenkassenrückvergütung: 2

Die Nennungen „Betreuung durch das Personal“ kann man den beiden Kategorien „Freundlichkeit“" und „Kompetenz des Personals“ zuordnen. Diese beiden Faktoren sind 
wie oben erwähnt bereits an der Spitze der Hitliste von Erfolgsfaktoren. Würde man diese 29 Nennungen noch dazuzählen, wären die beiden Personalfaktoren noch prominenter an erster Stelle.

Die Nennungen Preis und Öffnungszeiten sind bei der internen Ausscheidung der 12 Kriterien mit Kundenbefragungen ausgeschieden, tauchen hier jedoch wieder auf. Die wenigen Nennungen weisen darauf hin, dass sie gerechtfertigt weggelassen wurden.

Die 6 Nennungen „Physiotherapie/medizinische Betreuung im Haus“ möchte ich trotz der kleinen Anzahl kommentieren. Im Theorieteil wurde bereits darauf hingewiesen, dass sich die Fitnesscenterbranche im Produktelebenszyklus in der Reifephase befindet (vergleiche 4.1 Die Fitnesslandschaft Schweiz, Seite 23. Ein Charakteristikum der Reifephase ist, dass versucht wird, das Kerngeschäft auf benachbarte Geschäftsfelder auszudehnen. Im Bereich des Fitnesstrainings sind das Prävention und Rehabilitation. Es lässt sich ein Trend feststellen, dass Fitnesscenter versuchen, den Bereich Rehabilitation und medizinische Betreuung auch abzudecken. Im Gegenzug dazu versuchen die Physiotherapien und medizinischen Therapien, ihr Geschäftsfeld in Richtung Gesundheitstraining zu erweitern. Wer schlussendlich gewinnt, ist schwierig $\mathrm{zu}$ prognostizieren. Am erfolgversprechendsten ist sicher, wenn sich zwei Partner aus diesen unterschiedlichen Bereichen zusammenschliessen, jeweils das profunde Fachwissen der eigenen „Disziplin“ mitbringen und sich gegenseitig die Kunden im Grenzbereich zuweisen können.

Krankenkassen-Rückvergütung

Dies ist sicher ein relativ wichtiges Kriterium für den Kunden, da diese Rückvergütung 20-30\% des Abonnementspreises ausmachen kann. Ein solcher Rabatt kann auch von einem ,billigen“ Center kaum gegeben werden. Die wenigen Nennungen sind damit zu erklären, dass diese Untersuchung nur unter QUALITOP-zertifizierten Centern durchgeführt wurde. Wie schon im Theorieteil erwähnt, sind kommen alle Kunden von QUALITOP-Centern in den Genuss der Rückvergütung. Insofern ist dieser Faktor unter den zertifizierten Centern nicht relevant, im Vergleich mit nicht zertifizierten Centern spielt er aber sicher eine Rolle.

\subsection{Erfüllung der Kundenanforderungen}

Nach der Frage nach der Kundenwichtigkeit der einzelnen Faktoren wurden die Fitnesscenter-Manager gefragt, wie stark ihr Center diese Anforderungen erfüllt.

Die folgenden Antworten wurden dabei gegeben: 


\begin{tabular}{|l|l|l|l|l|}
\hline Faktor & $\begin{array}{l}\text { Nicht } \\
\text { erfüllt }\end{array}$ & $\begin{array}{l}+ \text { - } \\
\text { erfüllt }\end{array}$ & $\begin{array}{l}\text { Gut } \\
\text { erfüllt }\end{array}$ & $\begin{array}{l}\text { Sehr gut } \\
\text { erfüllt }\end{array}$ \\
\hline Breite des Angebots & $8 \%$ & $11 \%$ & $49 \%$ & $32 \%$ \\
\hline Moderne Maschinen und Geräte & $4 \%$ & $9 \%$ & $43 \%$ & $44 \%$ \\
\hline Sauberkeit der Nasszone & $0 \%$ & $10 \%$ & $33 \%$ & $57 \%$ \\
\hline Kinderbetreuung & $30 \%$ & $12 \%$ & $33 \%$ & $25 \%$ \\
\hline Versch. Abo-Möglichkeiten & $6 \%$ & $11 \%$ & $38 \%$ & $45 \%$ \\
\hline Zentrale Lage & $9 \%$ & $21 \%$ & $28 \%$ & $42 \%$ \\
\hline Anzahl Parkplätze & $10 \%$ & $24 \%$ & $27 \%$ & $39 \%$ \\
\hline Erreichbarkeit mit öV & $6 \%$ & $20 \%$ & $27 \%$ & $47 \%$ \\
\hline Freundlichkeit des Personals & $0 \%$ & $5 \%$ & $38 \%$ & $57 \%$ \\
\hline Kompetenz des Personals & $1 \%$ & $3 \%$ & $37 \%$ & $60 \%$ \\
\hline Kollegen trainieren hier & $5 \%$ & $29 \%$ & $51 \%$ & $15 \%$ \\
\hline Helle, hohe Räumlichkeiten & $9 \%$ & $9 \%$ & $29 \%$ & $53 \%$ \\
\hline
\end{tabular}

Tabelle 13-4 Erfüllung der Kundenanforderungen

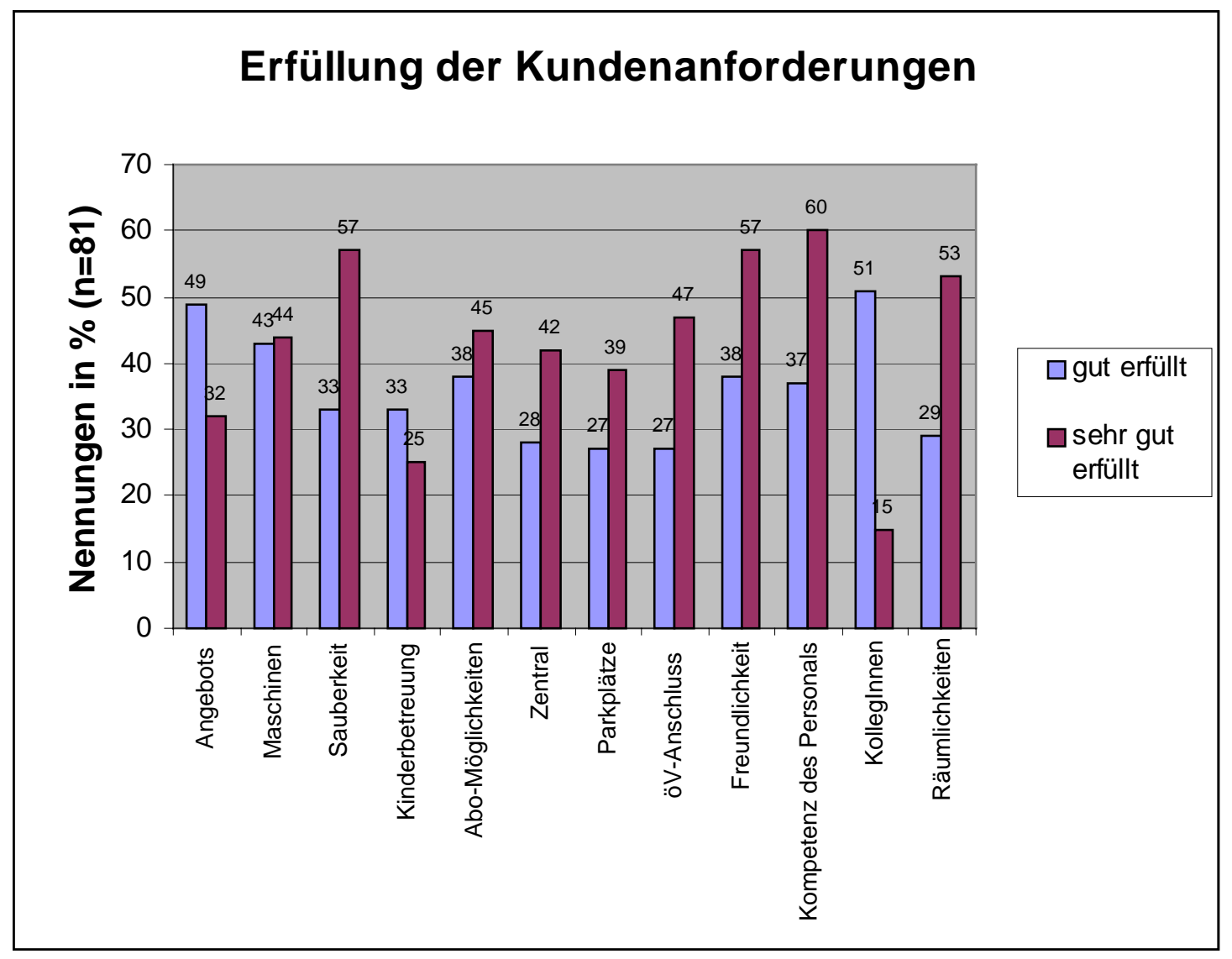

Abbildung 13-3 Erfüllung der Kundenanforderungen 
Die drei wichtigsten Faktoren Kompetenz und Freundlichkeit des Personals und Sauberkeit werden immerhin zu über 55\% sehr gut erfüllt. Es besteht jedoch immer noch ein grosses Verbesserungspotential.

Die folgende Gegenüberstellung zeigt, dass die drei Erfolgsfaktoren, die am besten erfüllt werden, auch die drei wichtigsten Faktoren aus Kundensicht sind.

\begin{tabular}{|l|l|l|l|}
\hline Rang & Faktor & $\begin{array}{l}\text { Wichtigkeit für den } \\
\text { Kunden }\end{array}$ & $\begin{array}{l}\text { Erfüllt durch } \\
\text { das Center }\end{array}$ \\
\hline 1 & Freundlichkeit des Personals & 3.88 & 3.52 \\
\hline 2 & Kompetenz des Personals & 3.74 & 3.54 \\
\hline 3 & Sauberkeit der Nasszone & 3.73 & 3.47 \\
\hline 4 & Anzahl Parkplätze & 3.16 & 2.96 \\
\hline 5 & Helle, hohe Räumlichkeiten & 3.10 & 3.27 \\
\hline 6 & Breite des Angebots & 2.99 & 3.06 \\
\hline 7 & Verschiedene Abonnements-Möglichkeiten & 2.94 & 3.21 \\
\hline 8 & Moderne Maschinen und Geräte & 2.91 & 3.28 \\
\hline 9 & Zentrale Lage & 2.79 & 3.04 \\
\hline 10 & Kollegen trainieren hier & 2.65 & 3.27 \\
\hline 11 & Kinderbetreuung & 2.46 & 2.53 \\
\hline 12 & Erreichbarkeit mit öffentl. Verkehrsmitteln & 2.42 & 3.15 \\
\hline
\end{tabular}

Tabelle 13-5 Wichtigkeit für Kunden und deren Erfüllung

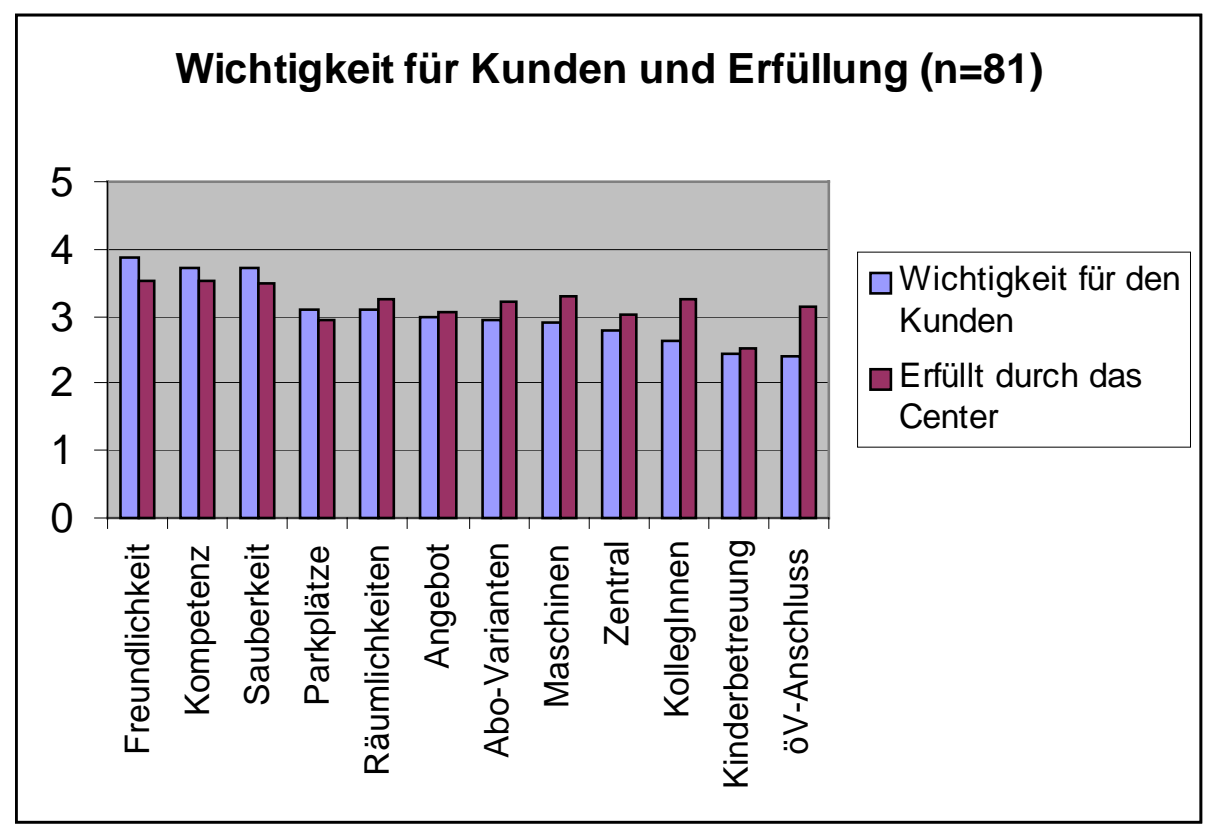

\section{Abbildung 13-4 Wichtigkeit der Faktoren und Erfüllung durch das Center}

Legende:

Wichtigkeit: 1=unwichtig, 2=ziemlich wichtig, 3=wichtig, 4=sehr wichtig

Erfüllung: 1=nicht erfüllt, $2=$ mehr oder weniger erfüllt, $3=$ gut erfüllt, $4=$ sehr gut erfüllt 
Generell kann gesagt werden, dass die Wichtigkeit aus Kundensicht und die Erfüllung durch die Center übereinstimmt.

Hier scheint in dem Sinne Einsicht und Umsetzung weniger zu divergieren als dies beim Führungsverhalten gezeigt werden konnte (vergleiche 11.3 Führungsverhalten: Wichtigkeit für den Erfolg und Zeitaufwand Seite 80 .

Die grössten Unterschiede bestehen beim Faktor „öV“. Hier ist offensichtlich das Bedürfnis der Kunden sehr tief, die Erfüllung durch die Center jedoch gut. Immerhin kann gesagt werden, dass dies keine „Verschleuderung von Ressourcen“ am falschen Ort ist, da der öffentliche Verkehr durch die Gemeinde und nicht durch das Center gefördert wird.

Diese Gegenüberstellung kann auch als Kontrollfrage für die Validität der ganzen Umfrage gewertet werden. Erfreulicherweise ergibt sich eine gute Validität.

\subsection{Zusammenfassung „Situation“}

- Die drei wichtigsten Erfolgsfaktoren aus Kundensicht sind:

- Freundlichkeit des Personals

- Kompetenz des Personals

- Sauberkeit der Nasszone

- Die drei Erfolgsfaktoren, die von den Centern am besten erfüllt werden, sind auch die drei wichtigsten aus Kundensicht. Der Erfüllungsgrad kann jedoch noch gesteigert werden. 


\section{Zusammenhänge zwischen Persönlichkeit und Führungs- verhalten}

\subsection{Genereller Zusammenhang von Persönlichkeit und Führungsverhalten}

Wie bereits bemerkt wurde, ergibt die Aufteilung in 16 verschiedene MBTI-Typen solch kleine Probandengruppen, dass Korrelationsanalysen mit einzelnen Typen kaum stichhaltige Antworten liefern können .

Deshalb werden nur die 4 Ausprägungen E-I, S-N, T-F und J-P mit den 12 verschiedenen Führungstätigkeiten korreliert. Aus den 48 Resultaten kann nur gerade bei fünf Ergebnissen eine Signifikanz unter 0.065 nachgewiesen werden. Dies ist der Fall bei:

Planen/koordinieren für die Ausprägung E-I

Planen/koordinieren für die Ausprägung J-P

Administration/Papierarbeit für die Ausprägung E-I

Motivieren/bestärken für die Ausprägung S-N

Akquisition/Marketing für die Ausprägung S-N

Grundsätzlich kann also festgestellt werden, dass man in dieser vorliegenden Untersuchung von den Persönlichkeitstypen her wenig signifikant auf das entsprechende Führungsverhalten in den 12 LOS-Kategorien schliessen kann. Dies mag einmal mehr mit den kleinen Gruppen zusammenhängen. Grundsätzlich geht man ja sonst bei der Verwendung des MBTI-Prsönlichkeitstests in der Berufsberatung oder im ManagementConsulting davon aus, dass die einzelnen Typen gewisse besondere Verhaltensweisen an den Tag legen, was man auch in vielen Studien nachweisen konnte (MYERS 1962, STRICKER \& ROSS 1968, MCCAULLEY 1978, MILLS 1983, LACY 1984, VELSOR \& CAMPBELL 1984).

In den folgenden Unterkapiteln werden die Resultate der einzelnen Ausprägungen im Detail vorgestellt. Es lässt sich zeigen, dass die MBTI-Typenbeschreibungen und das tatsächlich in dieser Untersuchung festgestellte Führungsverhalten meist tendenziell übereinstimmen, jedoch nicht wirklich signifikant sind. Dies hängt damit zusammen, dass die jeweiligen Ausprägungen nur eine Grunddisposition für das entsprechende Handeln darstellen und dieses Handeln nicht zwingend nach sich ziehen. 


\subsection{Führungsverhalten der Ausprägung E-I}

Die folgenden Resultate im Bereich der Ausprägung E-I konnten gemessen werden:

\begin{tabular}{|l|l|l|l|}
\hline Führungsverhalten & $\begin{array}{l}\text { Mehr Zeit aufge- } \\
\text { wendet durch E }\end{array}$ & $\begin{array}{l}\text { Mehr Zeit aufge- } \\
\text { wendet durch I }\end{array}$ & $\begin{array}{l}\text { Erwartung } \\
\text { MBTI-Test }\end{array}$ \\
\hline Planen, koordinieren & & X & I \\
\hline Personal führen & & $\mathrm{x}$ & $\mathrm{E}$ \\
\hline Ausbilden & & $\mathrm{x}$ & $\mathrm{E}$ \\
\hline Probleme lösen & & $\mathrm{x}$ & $\mathrm{I}$ \\
\hline Administration, Papierarbeit & & $\mathrm{X}$ & $\mathrm{I}$ \\
\hline Sitzungen, Info-Austausch & & $\mathrm{x}$ & $\mathrm{I}$ \\
\hline Überwachen, kontrollieren & $\mathrm{x}$ & & $\mathrm{E}$ \\
\hline Motivieren, bestärken & $\mathrm{x}$ & & $\mathrm{E}$ \\
\hline Disziplinieren, bestrafen & $\mathrm{x}$ & & $\mathrm{E}$ \\
\hline Akquisition, Marketing & $\mathrm{x}$ & & $\mathrm{E}$ \\
\hline Konfliktmanagement & & $\mathrm{x}$ & $\mathrm{E}$ \\
\hline Repräsentation & $\mathrm{x}$ & & $\mathrm{E}$ \\
\hline
\end{tabular}

Tabelle 14-1 Führungsverhalten der Ausprägung E-I

In $83 \%$ der Fälle stimmen die beobachteten Ergebnisse mit den Erwartungen überein.

Keine Übereinstimmung ist in den Bereichen Personal führen (Erwartung E, gemessen I) und Konfliktmanagement (Erwartung E, gemessen I) festzustellen.

Als Erklärung im Bereich Personalführung als auch im Bereich Konfliktmanagement kann angemerkt werden, dass E-Typen sicher ihr Personal ebenfalls führen und ebenfalls Konflikte lösen, wahrscheinlich durch einen direkteren Führungsstil jedoch weniger Zeit dafür aufwenden müssen. Diese Aussage müsste jedoch in einer eigenen Untersuchung mit einer grösseren Probandengruppe überprüft werden.

Eine signifikante Übereinstimmung ist für die beiden Verhaltensweisen Planen/Koordinieren $($ Sig. $=0.045)$ und Administration/Papierarbeit $($ Sig. $=0.032)$ nachzuweisen

Im Folgenden die detaillierte Auswertung:

Planen/Koordinieren (Sig. $=0.045)$

\begin{tabular}{|l|l|l|l|l|}
\hline & $<1 h$ & $1-3 h$ & $4-6 h$ & $>6 h$ \\
\hline $\mathrm{E}$ & $12 \%$ & $46 \%$ & $20 \%$ & $22 \%$ \\
\hline $\mathrm{I}$ & $0 \%$ & $30 \%$ & $40 \%$ & $30 \%$ \\
\hline
\end{tabular}

Tabelle 14-2 Zeitaufwand pro Woche für Planen/Koordinieren 


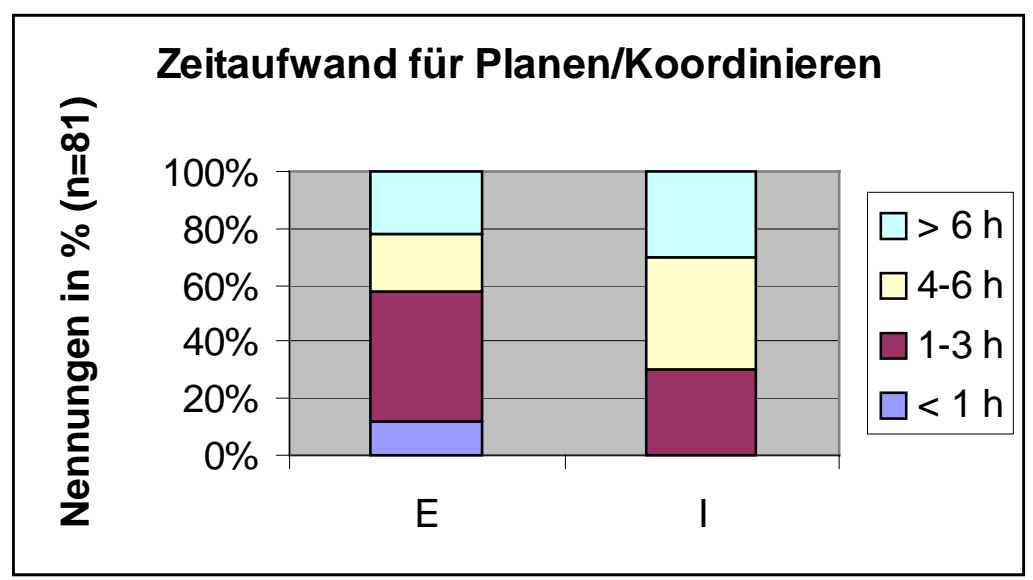

\section{Abbildung 14-1 Zeitaufwand pro Woche für Planen/Koordinieren}

$70 \%$ der I-Typen verwenden mehr als 4 Stunden für Planungs- und Koordinationsarbeiten (E: 42\%). Unter 4 Stunden kommen nur 30\% der I-Typen, jedoch $58 \%$ der E-Typen.

Administration, Papierarbeit (Sig. $=0.032)$

\begin{tabular}{|l|l|l|l|l|}
\hline & $<1 h$ & $1-3 h$ & $4-6 h$ & $>6 h$ \\
\hline $\mathrm{E}$ & $8 \%$ & $17 \%$ & $43 \%$ & $31 \%$ \\
\hline $\mathrm{I}$ & $0 \%$ & $11 \%$ & $31 \%$ & $58 \%$ \\
\hline
\end{tabular}

Tabelle 14-3 Zeitaufwand pro Woche für Administration

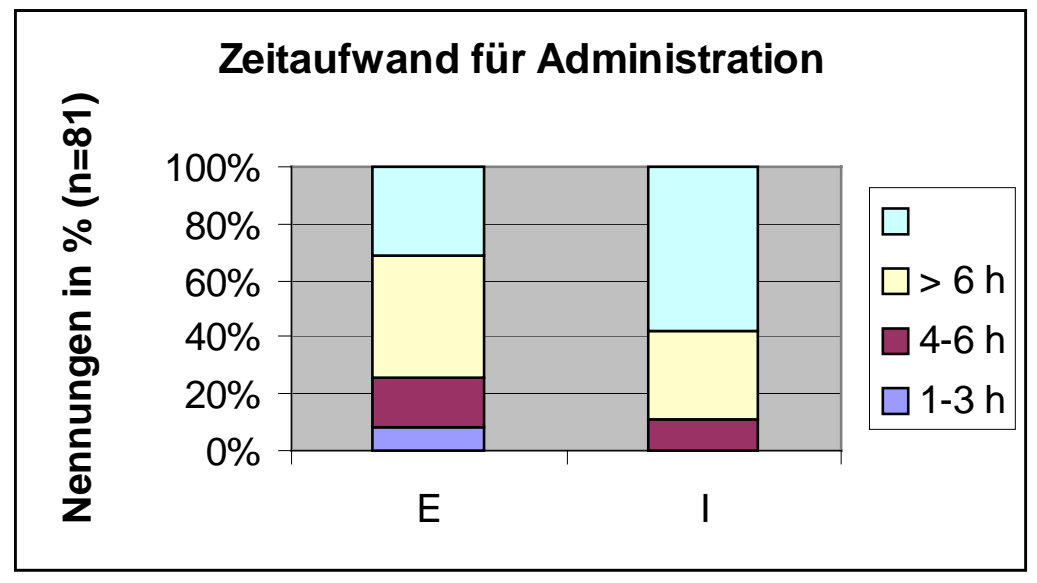

Abbildung 14-2 Zeitaufwand pro Woche für Administration

58\% der I-Typen verwenden mehr als sechs Stunden pro Woche für Administration (E: $31 \%$ ). Unter sechs Stunden kommen nur 42\% der I-Typen, jedoch 68\% der E-Typen. 


\subsection{Führungsverhalten der Ausprägung S-N}

Die folgenden Resultate im Bereich der Ausprägung S-N konnten gemessen werden:

\begin{tabular}{|l|l|l|l|}
\hline Führungsverhalten & Mehr Zeit aufge- & Mehr Zeit aufge- & Erwartung \\
\hline & wendet durch S & wendet durch N & MBTI-Test \\
\hline Planen, koordinieren & $\mathrm{x}$ & & $\mathrm{S}$ \\
\hline Personal führen & & $\mathrm{X}$ & $\mathrm{N}$ \\
\hline Ausbilden & $\mathrm{X}$ & & $\mathrm{S}$ \\
\hline Probleme lösen & & $\mathrm{X}$ & $\mathrm{N}$ \\
\hline Administration, Papierarbeit & & $\mathrm{X}$ & $\mathrm{S}$ \\
\hline Sitzungen, Info-Austausch & $\mathrm{x}$ & & $\mathrm{S}$ \\
\hline Überwachen, kontrollieren & $\mathrm{X}$ & & $\mathrm{S}$ \\
\hline Motivieren, bestärken & & $\mathrm{X}$ & $\mathrm{N}$ \\
\hline Disziplinieren, bestrafen & & & $\mathrm{X}$ \\
\hline Akquisition, Marketing & & $\mathrm{X}$ & $\mathrm{N}$ \\
\hline Konfliktmanagement & & $\mathrm{X}$ & $\mathrm{N}$ \\
\hline
\end{tabular}

Tabelle 14-4 Führungsverhalten der Ausprägung S-N

In 92\% der Fälle stimmen die beobachteten Ergebnisse mit den Erwartungen überein.

Keine Übereinstimmung ist im Bereich Administration/Papierarbeit (Erwartung $\mathrm{S}$, gemessen N) festzustellen. Papierarbeit beinhaltet nicht nur Informationsaufnahme (S$\mathrm{N}$-Ausprägung; hier wäre der N-Typ sicher schneller als der S-Typ), sondern auch Informationsverarbeitung (F-T-Ausprägung; da ist offensichtlich der N-Typ langsamer, da weniger organisiert).

Eine signifikante Übereinstimmung ist für die Führungstätigkeiten Motivieren/Bestärken $($ Sig. $=0.066)$ und Akquisition/Marketing $($ Sig. $=0.019)$ nachzuweisen.

Im Folgenden die detaillierte Auswertung:

Motivieren/Bestärken $($ Sig. $=0.066)$

\begin{tabular}{|l|l|l|l|l|}
\hline & $<1 h$ & $1-3 h$ & $4-6 h$ & $>6 h$ \\
\hline $\mathrm{S}$ & $25 \%$ & $56 \%$ & $14 \%$ & $5 \%$ \\
\hline $\mathrm{N}$ & $19 \%$ & $38 \%$ & $29 \%$ & $14 \%$ \\
\hline
\end{tabular}

Tabelle 14-5 Zeitaufwand pro Woche für Motivieren/Bestärken 


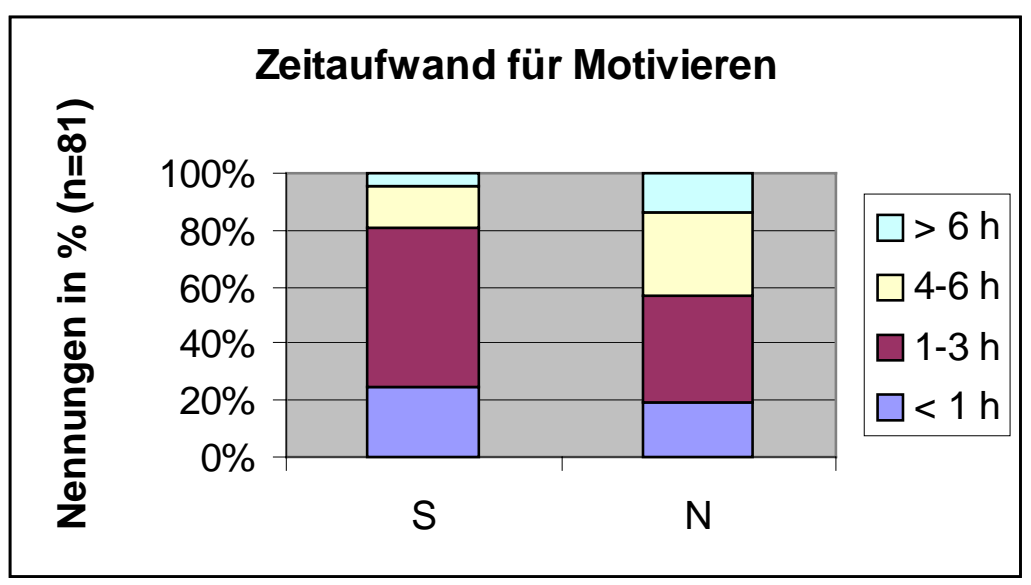

Abbildung 14-3 Zeitaufwand pro Woche für Motivieren

$81 \%$ der S-Typen verwenden weniger als drei Stunden pro Woche für Motivieren /Bestärken; nur 19\% investieren mehr als drei Stunden. Im Gegensatz dazu verwenden nur 57\% der N-Typen weniger als drei Stunden; $43 \%$ investieren mehr als drei Stunden.

Akquisition/Marketing (Sig. $=0.019)$

\begin{tabular}{|l|l|l|l|l|}
\hline & $<1 h$ & $1-3 h$ & $4-6 h$ & $>6 h$ \\
\hline $\mathrm{S}$ & $20 \%$ & $33 \%$ & $36 \%$ & $11 \%$ \\
\hline $\mathrm{N}$ & $4 \%$ & $36 \%$ & $21 \%$ & $39 \%$ \\
\hline
\end{tabular}

Tabelle 14-6 Zeitaufwand pro Woche für Akquisition/Marketing

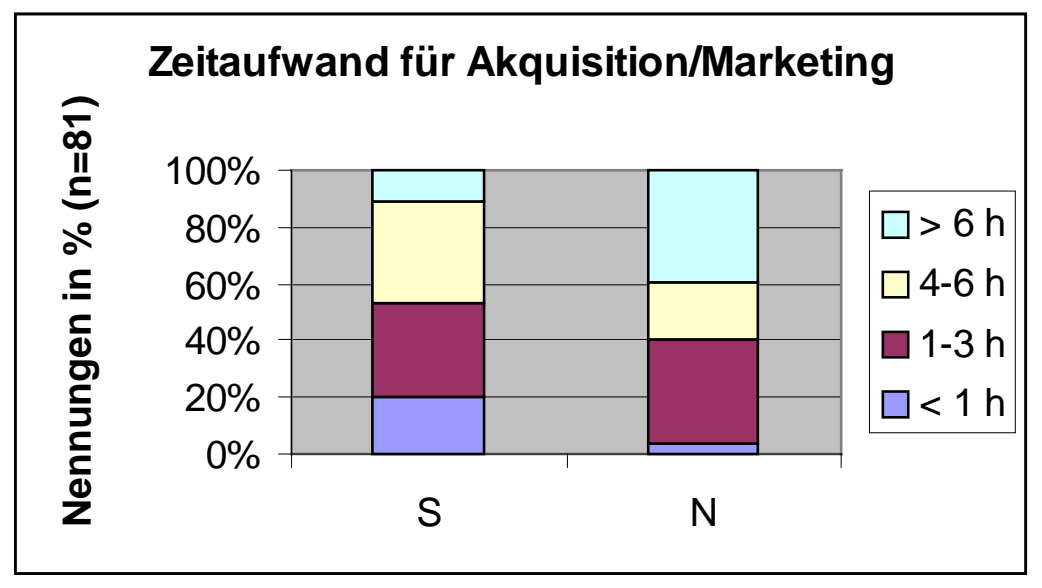

Abbildung 14-4 Zeitaufwand pro Woche für Akquisition/Marketing

53\% der S-Typen verwenden unter drei Stunden für Akquisition und Marketing, 47\% mehr als drei Stunden. Bei den N-Typen investieren nur $40 \%$ weniger als drei Stunden und $60 \%$ über drei Stunden. 


\subsection{Führungsverhalten der Ausprägung T-F}

Die folgenden Ergebnisse wurden im Bereich der Ausprägung T-F ermittelt:

\begin{tabular}{|l|l|l|l|}
\hline Führungsverhalten & Mehr Zeit aufge- & Mehr Zeit aufge- & Erwartung \\
\hline & wendet durch T & wendet durch F & MBTI-Test \\
\hline Planen, koordinieren & $\mathrm{x}$ & & $\mathrm{T}$ \\
\hline Personal führen & $\mathrm{x}$ & & $\mathrm{F}$ \\
\hline Ausbilden & $\mathrm{x}$ & & $\mathrm{T}$ \\
\hline Probleme lösen & $\mathrm{x}$ & $\mathrm{x}$ & $\mathrm{T}$ \\
\hline Administration, Papierarbeit & & & $\mathrm{F}$ \\
\hline Sitzungen, Info-Austausch & $\mathrm{x}$ & $\mathrm{x}$ & $\mathrm{T}$ \\
\hline Überwachen, kontrollieren & & & $\mathrm{T}$ \\
\hline Motivieren, bestärken & $\mathrm{X}$ & $\mathrm{x}$ & $\mathrm{F}$ \\
\hline Disziplinieren, bestrafen & & & \\
\hline Akquisition, Marketing & $\mathrm{x}$ & $\mathrm{x}$ & $\mathrm{T}$ \\
\hline Konfliktmanagement & & $\mathrm{F}$ \\
\hline
\end{tabular}

Tabelle 14-7 Führungsverhalten der Ausprägung T-F

In $75 \%$ der Fälle stimmen die beobachteten Ergebnisse mit den Erwartungen überein.

Keine Übereinstimmung ist in den Bereichen Personal führen (Erwartung F, gemessen $\mathrm{T}$ ), Überwachen/Kontrollieren (Erwartung $\mathrm{T}$, gemessen $\mathrm{F}$ ) und Motivieren/Bestärken (Erwartung F, gemessen T) festzustellen.

Personalführung: F-Typen messen wohl der Personalführung eine höhere Wichtigkeit zu als T-Typen, verwenden aber offenbar weniger Zeit dafür. Dies kann damit zusammenhängen, dass T-Typen wahrscheinlich aufwändigere Personalbeurteilungsund Personalfeedbacksysteme haben und damit mehr Zeit brauchen.

Überwachen/Kontrollieren: sehr kleine Differenz (0.095). Man könnte vereinfachend sagen, dass beide Typen gleich viel Zeit dafür einsetzen.

Motivieren/Bestärken: Hier kann eine ähnliche Erklärung wie bei der Personalführung vorgebracht werden. F-Typen messen dem Motivieren wohl einen höheren Stellenwert bei, wickeln das aber offensichtlich effizienter ab als T-Typen.

Eine signifikante Übereinstimmung ist für keine der Führungstätigkeiten nachzuweisen. 


\subsection{Führungsverhalten der Ausprägung J-P}

Die folgenden Ergebnisse wurden im Bereich der Ausprägung J-P ermittelt:

\begin{tabular}{|l|l|l|l|}
\hline Führungsverhalten & Mehr Zeit aufge- & Mehr Zeit aufge- & Erwartung \\
\hline & wendet durch J & wendet durch P & MBTI-Test \\
\hline Planen, koordinieren & X & & J \\
\hline Personal führen & $\mathrm{X}$ & & J \\
\hline Ausbilden & $\mathrm{X}$ & $\mathrm{X}$ & $\mathrm{J}$ \\
\hline Probleme lösen & & & $\mathrm{P}$ \\
\hline Administration, Papierarbeit & $\mathrm{X}$ & & $\mathrm{J}$ \\
\hline Sitzungen, Info-Austausch & $\mathrm{x}$ & & $\mathrm{J}$ \\
\hline Überwachen, kontrollieren & $\mathrm{X}$ & $\mathrm{X}$ & $\mathrm{J}$ \\
\hline Motivieren, bestärken & & & $\mathrm{P}$ \\
\hline Disziplinieren, bestrafen & & $\mathrm{X}$ & $\mathrm{X}$ \\
\hline Akquisition, Marketing & & $\mathrm{X}$ & $\mathrm{J}$ \\
\hline Konfliktmanagement & & $\mathrm{P}$ \\
\hline
\end{tabular}

Tabelle 14-8 Führungsverhalten der Ausprägung J-P

In 93\% der Fälle stimmen die beobachteten Ergebnisse mit den Erwartungen überein.

Keine Übereinstimmung ist im Bereich Akquisition/Marketing (Erwartung J, gemessen P) festzustellen. Marketing ist an sich eine planerische Tätigkeit und deshalb klar Jlastig. Offensichtlich investieren hier P-Typen aber mehr Zeit. Vielleicht weil sie sich der Wichtigkeit des Marketings bewusst sind und darum absichtlich ein Gegengewicht zu ihren Präferenzen setzen.

Eine signifikante Übereinstimmung ist für die Führungstätigkeit Planen/Koordinieren nachzuweisen.

Planen/Koordinieren (Sig. $=0.034)$

\begin{tabular}{|l|l|l|l|l|}
\hline & $<1 h$ & $1-3 h$ & $4-6 h$ & $>6 h$ \\
\hline $\mathrm{J}$ & $3 \%$ & $37 \%$ & $33 \%$ & $27 \%$ \\
\hline $\mathrm{P}$ & $17 \%$ & $50 \%$ & $13 \%$ & $20 \%$ \\
\hline
\end{tabular}

Tabelle 14-9 Aufgewendete Zeit pro Woche für Planen/Koordinieren 


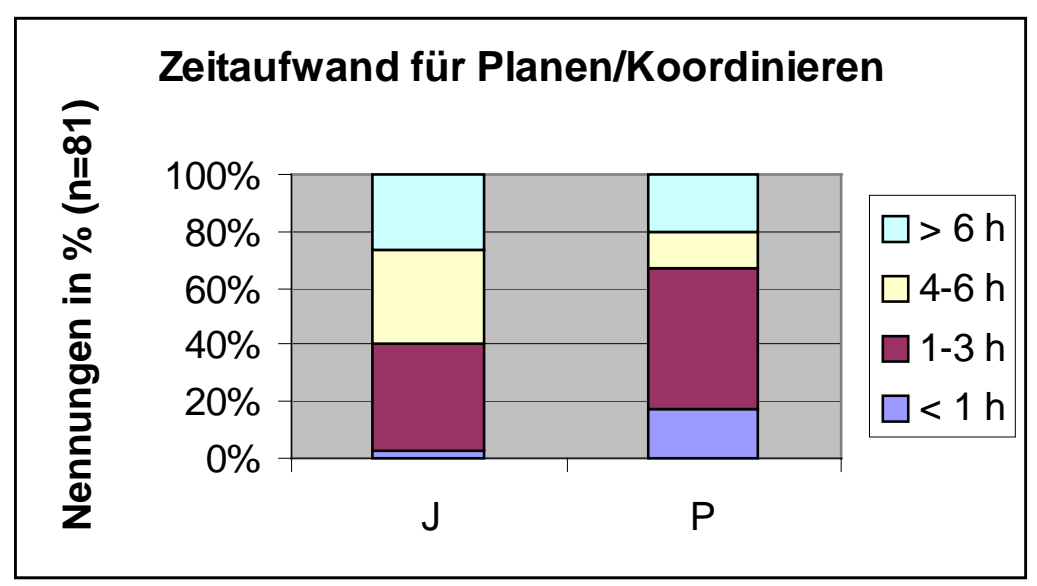

\section{Abbildung 14-5 Zeitaufwand pro Woche für Planen/Koordinieren}

40\% der J-Typen verwenden weniger als 3 Stunden für Planung und Koordination, 60\% mehr als drei Stunden. Im Gegensatz dazu verwenden über 67\% der P-Typen weniger als drei Stunden für Planung, nur 33\% investieren über drei Stunden.

Dieses Resultat erstaunt wenig, da der J-Typ als klassischer Planer hier sicher viel Zeit investiert. P-Typen nehmen die Sache wie es kommt, müssen aber deshalb im Bereich Konfliktmanagement und Probleme lösen mehr Zeit investieren.

\subsection{Zeitaufwand-Extremwerte für die einzelnen Führungstätigkeiten}

Mangels Aussagekraft wird hier darauf verzichtet, für jede Führungstätigkeit und jeden Typen den jeweiligen Zeitaufwand aufzulisten. Im Folgenden sollen nur die jeweiligen Extremwerte notiert werden.

\begin{tabular}{|l|l|l|l|}
\hline Führungstätigkeit & $\begin{array}{l}\text { Minimalwert } \\
(\text { Typ })\end{array}$ & Maximalwert (Typ) & Differenz \\
\hline Planen, koordinieren & $2.37(\mathrm{P})$ & $3.00(\mathrm{I})$ & 0.63 \\
\hline Personal führen & $2.43(\mathrm{P})$ & $2.65(\mathrm{I})$ & 0.22 \\
\hline Ausbilden & $1.97(\mathrm{P})$ & $2.10(\mathrm{~J})$ & 0.13 \\
\hline Probleme lösen & $2.19(\mathrm{~F})$ & $2.44(\mathrm{I})$ & 0.25 \\
\hline Administration, Papierarbeit & $2.97(\mathrm{~S})$ & $3.47(\mathrm{I})$ & 0.50 \\
\hline Sitzungen, Info-Austausch & $2.07(\mathrm{P})$ & $2.30(\mathrm{I})$ & 0.23 \\
\hline Überwachen, kontrollieren & $2.00(\mathrm{I})$ & $2.30(\mathrm{E})$ & 0.30 \\
\hline Motivieren, bestärken & $2.00(\mathrm{~S})$ & $2.38(\mathrm{~N})$ & 0.38 \\
\hline Disziplinieren, bestrafen & $1.07(\mathrm{~F})$ & $1.17(\mathrm{~T})$ & 0.10 \\
\hline Akquisition, Marketing & $2.39(\mathrm{~S})$ & $2.93(\mathrm{~N})$ & 0.54 \\
\hline Konfliktmanagement & $1.37(\mathrm{~S})$ & $1.66(\mathrm{~N})$ & 0.29 \\
\hline Repräsentation & $1.55(\mathrm{I})$ & $1.91(\mathrm{~N})$ & 0.36 \\
\hline
\end{tabular}

Legende: $1=<1 \mathrm{~h}, 2=1-3 \mathrm{~h}, 3=3-6 \mathrm{~h}, 4=>6 \mathrm{~h}$ pro Woche Zeitaufwand 


\section{Tabelle 14-10 Extreme Zeitaufwand pro Führungstätigkeit}

Weniger als 0.25 Differenz zwischen den Extremwerten gibt es bei folgenden Führungstätigkeiten:

- Personal führen

- Ausbilden

- Probleme lösen

- Sitzungen, Informationsaustausch

- Disziplinieren, bestrafen

Für diese Tätigkeiten wenden offensichtlich alle Typen in etwa gleich viel Zeit auf. Erstaunlich ist dies vor allem für Sitzungen/Informationsaustausch. Hier erwartet man eigentlich, dass sich erhebliche Unterschiede zwischen den einzelnen Typen ergeben.

Zwischen 0.25 und 0.49 Differenz gibt es bei den folgenden Tätigkeiten:

- Überwachen, kontrollieren

- Motivieren, bestärken

- Konfliktmanagement

- Repräsentation, soziale Verpflichtungen

Über 0.5 Punkte Differenz ergeben sich bei:

- Planen, koordinieren

- Administration, Papierarbeit

- Akquisition, Marketing

Die beiden ersten Tätigkeiten waren beinahe zu erwarten. In die Planung und die Administration kann man eigentlich beliebig viel investieren; sie können immer noch perfekter gemacht werden. Je nach Typ zeigen sich hier grosse Unterschiede. Interessant ist, dass beide Male der I-Typ sowohl in Planung als auch in Administration am meisten Zeit investiert (auch im Bereich Marketing ist der I-Typ bei denen, die sehr viel investieren).

Bei Akquisition/Marketing kann der grosse Unterschied auch daher rühren, dass man je nach Ansicht mehr oder weniger unter Marketing verstehen kann. Was für den einen Centerleiter noch Administration ist, wird durch den anderen bereits als Marketing verstanden. Erwähnenswert ist hier, dass beide Male sowohl bei Administration als auch bei Marketing der S-Typ am wenigsten Zeit investiert (bei der Planung befindet er sich im Mittelfeld).

Zusammenfassend kann Folgendes festgestellt werden:

Minimaler Zeitaufwand: Maximaler Zeitaufwand:

P-Typ: $4 \mathrm{x} \quad$ I-Typ: $5 \mathrm{x}$

S-Typ: $4 \mathrm{x} \quad$ N-Typ: $4 \mathrm{x}$

I-Typ: $2 \mathrm{x} \quad$ E-/T-/J-Typ: je $1 \mathrm{x}$

F-Typ: $2 \mathrm{x}$

Die Häufung bei einzelnen Typen fällt auf. Ob damit aber P- und S-Typen eher als Minimalisten, I- und N-Typen als Arbeitstiere bezeichnet werden können, ist mehr als fraglich. Auch hier müsste eine detailliertere Untersuchung genauere Daten erheben. 
14.7 Zusammenfassung „Persönlichkeit $\leftarrow \rightarrow$ Führungsverhalten“

- Einzelne signifikante Zusammenhänge zwischen Persönlichkeit und Führungsverhalten sind nachweisbar.

- Die Ergebnisse decken sich mit den Erwartungen aus den MBTI-Profilen.

- I-Typen verwenden signifikant mehr Zeit für Planen/Koordinieren und Administration/Papierarbeit als E-Typen.

- N-Typen verwenden signifikant mehr Zeit für Motivieren/Bestärken und Akquisition/Marketing als S-Typen.

- J-Typen verwenden signifikant mehr Zeit für Planen/Koordinieren und Administration/Papierarbeit als P-Typen. 


\section{Zusammenhänge zwischen Führungsverhalten und Erfolg}

\subsection{Führungsverhalten und Erfolg als Ganzes}

Korreliert man die einzelnen Führungstätigkeiten mit Erfolg (Summe der persönlichen Erfolgsbeurteilung, Variablen 328 - 333), so ergeben sich folgende Signifikanzen, bzw. eben Nicht-Signifikanzen:

\begin{tabular}{|l|l|}
\hline Führungstätigkeit & $\begin{array}{l}\text { Signifikanz mit } \\
\text { Erfolg }\end{array}$ \\
\hline Planen, koordinieren & 0.348 \\
\hline Personal führen & 0.960 \\
\hline Ausbilden & 0.403 \\
\hline Probleme lösen & 0.648 \\
\hline Administration, Papierarbeit & 0.478 \\
\hline Sitzungen, Info-Austausch & 0.667 \\
\hline Überwachen, kontrollieren & 0.764 \\
\hline Motivieren, bestärken & 0.482 \\
\hline Disziplinieren, bestrafen & 0.372 \\
\hline Akquisition, Marketing & 0.696 \\
\hline Konfliktmanagement & 0.962 \\
\hline Repräsentation & 0.808 \\
\hline
\end{tabular}

Tabelle 15-1 Erfolgs-Signifikanz der einzelnen Führungstätigkeiten I

Es lassen sich keine signifikanten Zusammenhänge zwischen den einzelnen Führungstätigkeiten und Erfolg nachweisen.

Tendenziell lässt sich aussagen, dass es sich eher lohnt, bei folgenden Führungstätigkeiten Zeit zu investieren (Signifikanz < 0.5):

- Planen, koordinieren

- Disziplinieren, bestrafen

- Ausbilden

- Administration, Papierarbeit

- Motivieren, bestärken

\subsection{Führungsverhalten und Erfolg zweifach kategorisiert}

Teilt man die Variable Erfolg auf in die zwei Unterkategorien „weniger erfolgreich“ (Nennungen in den beiden Kategorien ,nicht erfolgreich“ und „mässig erfolgreich“) und „mehr erfolgreich“ (Nennungen in den beiden Kategorien „erfolgreich“ und „sehr erfolgreich") so ergeben sich noch etwas klarere Resultate: 


\begin{tabular}{|l|l|}
\hline Führungstätigkeit & $\begin{array}{l}\text { Signifikanz mit „weniger } \\
\text { /mehr erfolgreich“ }\end{array}$ \\
\hline Planen, koordinieren & 0.365 \\
\hline Personal führen & 0.805 \\
\hline Ausbilden & 0.077 \\
\hline Probleme lösen & 0.377 \\
\hline Administration, Papierarbeit & 0.203 \\
\hline Sitzungen, Info-Austausch & 0.942 \\
\hline Überwachen, kontrollieren & 0.743 \\
\hline Motivieren, bestärken & 0.807 \\
\hline Disziplinieren, bestrafen & 0.468 \\
\hline Akquisition, Marketing & 0.750 \\
\hline Konfliktmanagement & 0.961 \\
\hline
\end{tabular}

Tabelle 15-2 Erfolgs-Signifikanz der einzelnen Führungstätigkeiten II

Es ergeben sich noch verstärkte Tendenzen für Erfolg bei den Führungstätigkeiten Ausbilden, Administration, Planung und Probleme lösen, wobei Ausbilden sogar knapp signifikant herauskommt $($ Signifikanz $=0.077)$.

15.3 Extremwerte Führungsverhalten und Erfolg (vierfach kategorisiert)

\begin{tabular}{|l|c|c|c|}
\hline Führungstätigkeit & \multicolumn{2}{|l|}{$\begin{array}{l}\text { Zeitaufwand für das } \\
\text { Erfolgs-Resultat: }\end{array}$} & Differenz \\
\hline & $\begin{array}{l}\text { Mässig } \\
\text { erfolgreich }\end{array}$ & $\begin{array}{l}\text { Sehr } \\
\text { erfolgreich }\end{array}$ & \\
\hline Planen, koordinieren & 2.48 & 2.83 & 0.35 \\
\hline Personal führen & 2.60 & 3.17 & 0.57 \\
\hline Ausbilden & 2.32 & 3.50 & 0.82 \\
\hline Probleme lösen & 2.24 & 2.67 & 0.43 \\
\hline Administration, Papierarbeit & 2.96 & 2.67 & -0.19 \\
\hline Sitzungen, Info-Austausch & 2.20 & 2.33 & 0.13 \\
\hline Überwachen, kontrollieren & 2.28 & 2.67 & 0.39 \\
\hline Motivieren, bestärken & 2.24 & 2.17 & -0.07 \\
\hline Disziplinieren, bestrafen & 1.09 & 1.33 & 0.24 \\
\hline Akquisition, Marketing & 2.72 & 2.50 & -0.22 \\
\hline Konfliktmanagement & 1.56 & 2.20 & 0.64 \\
\hline Repräsentation & 1.88 & 2.33 & 0.45 \\
\hline
\end{tabular}

Legende:

Arbeitsaufwand: $1=<1 \mathrm{~h}, 2=1-3 \mathrm{~h}, 3=3-6 \mathrm{~h}, 4=>6 \mathrm{~h}$ pro Woche

Tabelle 15-3 Extreme Führungsverhalten und Erfolg 
Fast bei allen Führungstätigkeiten lässt sich feststellen, dass mehr Zeit in die jeweilige Tätigkeit investieren jeweils auch mehr Erfolg bringt. Einzig bei den Führungsverhalten Administration und Motivieren trifft dies nicht zu.

Am grössten sind die Unterschiede zwischen mässig erfolgreichen und sehr erfolgreichen Center-Managerinnen bei der investierten Zeit in die Führungstätigkeiten

- Ausbilden

- Konfliktmanagement

- Personal führen

Offensichtlich lohnt es sich in diesen drei Bereichen am meisten, Zeit zu investieren.

\subsection{Führungsverhalten des sehr erfolgreichen Managers}

Aufgrund der ermittelten Ergebnisse stellt sich das Führungsverhalten des sehr erfolgreichen Fitnesscenter-Managers wie folgt dar:

\begin{tabular}{|l|l|l|}
\hline Rang & Führungstätigkeit & $\begin{array}{l}\text { Zeitaufwand } \\
\text { pro Woche }\end{array}$ \\
\hline 1 & Personal führen & 3.17 \\
\hline 2 & Planen, koordinieren & 2.83 \\
\hline 3 & Probleme lösen & 2.67 \\
\hline & Administration, Papierarbeit & 2.67 \\
\hline & Überwachen, kontrollieren & 2.67 \\
\hline 6 & Ausbilden & 2.50 \\
\hline & Akquisition, Marketing & 2.50 \\
\hline 8 & Sitzungen, Informationsaustausch & 2.33 \\
\hline & Repräsentation, Akquisition & 2.33 \\
\hline 10 & Konfliktmanagement & 2.20 \\
\hline 11 & Motivieren, bestärken & 2.17 \\
\hline 12 & Disziplinieren, bestrafen & 1.33 \\
\hline
\end{tabular}

Legende:

Arbeitsaufwand: $1=<1 \mathrm{~h}, 2=1-3 \mathrm{~h}, 3=3-6 \mathrm{~h}, 4=>6 \mathrm{~h}$ pro Woche

Tabelle 15-4 Führungstätigkeiten des sehr erfolgreichen Managers

Generell muss festgestellt werden, dass sich mit dieser Untersuchung wenig griffige oder gar signifikante Zusammenhänge zwischen Führungsverhalten und Erfolg aufzeigen lassen.

Es wurden im Rahmen dieser Studie nur quantitative Werte erhoben (wieviel Zeit setzen Sie für die jeweiligen Führungstätigkeiten ein?), da qualitative Erhebungen den Rahmen bei weitem gesprengt hätten. Allein die quantitativen Abweichungen lassen wenig 
Schlüsse von Führungsverhalten auf Erfolg zu. Das wiederum bedeutet, dass ein grosser Teil der Erklärungen für den Erfolg im Bereich der Qualität des Führungsverhaltens gesucht werden muss, was zum Beispiel in weiterführenden Untersuchungen abgeklärt werden könnte.

Die erzielten Resultate sind aber grundsätzlich recht plausibel. Im Allgemeinen geht man ja auch davon aus, dass man mit mehr Arbeiten auch mehr bewegt, dass es aber noch um einiges wichtiger ist, wie qualitativ gut oder wie effektiv die Arbeit ist. Damit schliesst sich der Kreis zur Diskussion über Effizienz versus Effektivität im Kapitel Querverweis.

Beide Effekte, dass man mit mehr Arbeiten mehr erreicht und dass man mit effektiverem Arbeiten mehr erreicht, konnten gezeigt werden. Im Bereich der Quantität sind mit den vorhandenen quantitativen Daten Aussagen möglich, im Bereich der Qualität müsste mit qualitativer Datenerhebung gearbeitet werden, was viel aufwendiger wäre und kaum mehr repräsentativ realisiert werden könnte.

In dieser Arbeit wurde der Anspruch an Repräsentativität gestellt, was aber wiederum den qualitativen Ansatz ausschloss.

In diesem Sinne konnten keine weitergehenden Befunde als die gemachten erwartet werden.

\subsection{Zusammenfassung „Führungsverhalten $\leftarrow \rightarrow$ Erfolg}

- Es konnten keine signifikanten Zusammenhänge zwischen Führungstätigkeiten und Erfolg nachgewiesen werden.

- Die erfolgversprechendsten Tätigkeiten sind

- Ausbilden

- Administration

- Planung

- Probleme lösen

- Sehr erfolgreiche Center-Manager investieren markant mehr Zeit als ihre mässig erfolgreichen Kolleginnen in folgende Führungsaufgaben:

- Ausbilden

- Konfliktmanagement

- Personalführung

- Mit der vorhandenen Untersuchungsanlage kann nur über die quantitativen, nicht aber über die qualitativen Unterschiede der einzelnen Führungstätigkeiten und den damit verbundenen Auswirkungen auf den Erfolg ausgesagt werden.

- Der Erfolg wird mehr (und wahrscheinlich sogar vor allem) durch das qualitative Führungsverhalten (wie?) als durch das quantitative Führungsverhalten (wieviel?) beeinflusst. 


\section{Zusammenhang zwischen Persönlichkeit und Erfolg}

\subsection{Rangliste der 16 Einzeltypen}

Folgende Rangliste ergibt sich, wenn die 16 MBTI-Typen nach ihrem selbstbeurteilten Erfolg sortiert werden:

\begin{tabular}{|c|c|c|c|}
\hline Rang & Typ & Erfolgswert & Anzahl Probanden \\
\hline \multicolumn{4}{|c|}{ Erfolgswert über 2.9} \\
\hline 1 & ISTP & 3.0 & 1 \\
\hline 2 & ESTP & 2.98 & 3 \\
\hline \multirow[t]{2}{*}{3} & ESTJ & 2.97 & 12 \\
\hline & ESFP & 2.97 & 3 \\
\hline \multicolumn{4}{|c|}{ Erfolgswert über 2.7} \\
\hline 5 & ENTP & 2.80 & 10 \\
\hline 6 & ENTJ & 2.78 & 9 \\
\hline 7 & ISTJ & 2.75 & 7 \\
\hline \multicolumn{4}{|c|}{ Erfolgswert über 2.5} \\
\hline 8 & INFP & 2.64 & 2 \\
\hline 9 & INTJ & 2.58 & 6 \\
\hline 10 & ESFJ & 2.55 & 7 \\
\hline 11 & ENFJ & 2.52 & 7 \\
\hline \multicolumn{4}{|c|}{ Erfolgswert über 2.0} \\
\hline 12 & ENFP & 2.41 & 10 \\
\hline 13 & ISFP & 2.33 & 1 \\
\hline 14 & INTP & 2.17 & 1 \\
\hline 15 & ISFJ & 2.08 & 2 \\
\hline 16 & INFJ & - & 0 \\
\hline
\end{tabular}

Legende

Erfolgswert: 2 = mässig erfolgreich, 3 = erfolgreich, 4 = sehr erfolgreich

Summe der persönlichen Einschätzungen in den Bereichen Mitgliederzahlen, Auslastungsschwankungen, Kundenzufriedenheit, Gewinn, Gesamtkapitalrentabilität.

Tabelle 16-1 Rangliste der 16 MBTI-Typen nach Erfolg 


\subsection{Rangliste der Kerntypen}

Reduziert man die 16 Typen auf die 4 Kerntypen, so ergeben sich folgende Resultate:

\begin{tabular}{|l|l|l|l|}
\hline Rang & Kerntyp & Erfolgswert & Anzahl Probanden \\
\hline 1 & ST & 2.91 & 23 \\
\hline 2 & NT & 2.72 & 26 \\
\hline 3 & SF & 2.56 & 13 \\
\hline 4 & NF & 2.47 & 19 \\
\hline
\end{tabular}

\section{Legende}

Erfolgswert: 2 = mässig erfolgreich, 3 = erfolgreich, 4 = sehr erfolgreich

Summe der persönlichen Einschätzungen in den Bereichen Mitgliederzahlen, Auslastungsschwankungen, Kundenzufriedenheit, Gewinn, Gesamtkapitalrentabilität.

\section{Tabelle 16-2 Rangliste der MBTI-Kerntypen nach Erfolg}

Isoliert man innerhalb der 4 Kerntypen noch die einzelnen Komponenten, so erhält man folgende Übersicht:

\begin{tabular}{|l|l|l|l|}
\hline Rang & Komponente & Erfolgswert & Anzahl Probanden \\
\hline 1 & $\mathrm{~T}$ & 2.81 & 49 \\
\hline 2 & $\mathrm{~S}$ & 2.78 & 36 \\
\hline 3 & $\mathrm{~N}$ & 2.60 & 45 \\
\hline 4 & $\mathrm{~F}$ & 2.50 & 32 \\
\hline
\end{tabular}

\section{Legende}

Erfolgswert: 2 = mässig erfolgreich, 3 = erfolgreich, $4=$ sehr erfolgreich

Summe der persönlichen Einschätzungen in den Bereichen Mitgliederzahlen, Auslastungsschwankungen, Kundenzufriedenheit, Gewinn, Gesamtkapitalrentabilität.

Tabelle 16-3 Rangliste der Kerntypen-Komponenten nach Erfolg 


\subsection{Rangliste der 4 Ausprägungen nach Erfolg}

Korreliert man die 4 Ausprägungen E-I, S-N, T-F und J-P mit der persönlichen Einschätzung von Erfolg, so erhält man folgende Ergebnisse:

\begin{tabular}{|l|l|l|}
\hline Ausprägung & Korrelation & Signifikanz \\
\hline E-I & -.1299 & 0.251 \\
\hline S-N & -.1302 & 0.250 \\
\hline T-F & -.2184 & 0.052 \\
\hline J-P & -.0061 & 0.957 \\
\hline
\end{tabular}

\section{Tabelle 16-4 Korrelationen der 4 MBTI-Ausprägungen mit Erfolg}

Die obigen Zahlen können folgendermassen interpretiert werden:

Der E-Typ ist in unserer Untersuchung tendenziell erfolgreicher als der I-Typ.

Der S-Typ ist tendenziell erfolgreicher als der N-Typ.

Der T-Typ ist signifikant erfolgreicher als der F-Typ.

Der J-Typ ist eher erfolgreich als der P-Typ.

Der Prototyp des erfolgreichen Fitnesscenter-Managers ist folglich der ESTJ-Typ.

Interessanterweise ist also der in unserer Untersuchung am meisten vertretene Persönlichkeits-Typ (ESTJ) unter den erhobenen Centern am erfolgreichsten. Ob da bereits etwas von Survival of the Fittest durchschimmert, wäre aber vermessen zu sagen.

Bei der Betrachtung der Kerntypen stellt man ebenfalls fest, dass der ST-Typ erfolgsmässig mit grossem Abstand vor den anderen Typen kommt.

Bei der isolierten Betrachtung der einzelnen Kerntypen-Komponenten zeigt sich, dass die T-Komponente am meisten zum Erfolg beisteuert, gefolgt von der S-Komponente, der N- und schlussendlich der F-Komponente.

\subsection{Der Erfolgstyp ESTJ}

Im Folgenden soll etwas genauer auf den ESTJ-Typen eingegangen werden.

Dass der ESTJ-Typ als Erfolgstyp bezeichnet werden kann, deckt sich mit den Erkenntnissen der beiden vorangehenden Unterkapiteln. In der Rangliste aller 16 Typen ist der ESTJ-Typ zwar „nur“ gerade auf Rang drei, die beiden vor ihm liegenden Typen (ISTP und ESTP) sind jedoch nur mit einem oder drei Probanden in der Stichprobe vertreten und kaum repräsentativ. Der ESTJ-Typ mit 12 Probanden in der Stichprobe stellt die grösste Gruppe dar. Das Resultat kann also eher als stichhaltig angesehen werden.

Zusammenhänge von ESTJ-Typen und Manager-Persönlichkeiten konnten auch in anderen Studien nachgewiesen werden.

Im Vergleich der beiden Persönlichkeitsinventare MBTI und Study of Value (ALLPORT et al., 1960) konnte MYERS (1962) mit jeweils 1351 und 877 Probanden 
nachweisen, dass die ALLPORTsche Ausprägung „Economic“ - was gut zu unserem Manager passt - jeweils signifikant $(p<0.1)$ oder stark signifikant $(p<0.01)$ mit den Ausprägungen E, S, T und J korrelierte.

MCCAULLEY (1978) zeigte in drei Studien mit jeweils 66, 484 und 645 Probanden beiden Geschlechts, dass die vier Ausprägungen E, S, T und J jeweils stark signifikant ( $\mathrm{p}<0.001)$ mit Leadership-Potential korrelieren.

LACY (1984) zeigte, dass von zwei Stichproben mit je 912 und 848 Probanden die Berufsgattungen Army Officer, Navy Officer, Department Store Manager, Marketing Executive, Agri Business Manager, Chamber of Commerce Executive, Credit manager, Banker und Accountant jeweils mit mindestens drei der vier Ausprägungen signifikant oder stark signifikant korrelierten.

Die mit dieser Untersuchung erzielten Resultate können folglich einmal mehr als plausibel und der Fragebogen als valid bezeichnet werden.

Die Resultate einer Persönlichkeits-Erhebung unter Sportlehrer-Studierenden (SCHMID, 1999 b) seien an dieser Stelle vorgestellt:

\begin{tabular}{|l|l|l|}
\hline MBTI-Typ & Anzahl absolut & In $\%$ \\
\hline ISTJ & 12 & 16 \\
\hline ISTP & 3 & 4 \\
\hline ESTP & 2 & 3 \\
\hline ESTJ & 15 & 20 \\
\hline ISFJ & 5 & 7 \\
\hline ISFP & 0 & \\
\hline ESFP & 3 & 4 \\
\hline ESFJ & 5 & 7 \\
\hline INFJ & 2 & 3 \\
\hline INFP & 1 & 1 \\
\hline ENFP & 13 & 17 \\
\hline ENFJ & 4 & 6 \\
\hline INTJ & 2 & 3 \\
\hline INTP & 0 & 0 \\
\hline ENTP & 4 & 6 \\
\hline ENTJ & 4 & 6 \\
\hline Total & 75 & 100 \\
\hline & &
\end{tabular}

Tabelle 16-5 Persönlichkeitstypen von Sportstudierenden

Der ESTJ-Typ ist der am meisten verbreitete Typ unter Sportstudierenden. Sportler sind meist sehr leistungs- und erfolgsorientiert. Die Zusammenhänge mit dem Erfolgstypen ESTJ ist deshalb nicht erstaunlich.

Die Funde von SCHMID (1999 b) decken sich übrigens mit den Ergebnissen von VELSOR und CAMPBELL (1984), die von 1228 Studierenden bei den 
Sportstudierenden ebenfalls in allen vier Ausprägungen E, S, T und J sehr signifikante $(\mathrm{p}<0.01)$ Zusammenhänge aufzeigten.

Man kann also Sportstudierende als gutes Rekrutierungsfeld für zukünftige erfolgreiche Manager betrachten. Immerhin 20\% sind ESTJ-Typen.

Der ESTJ-Typ wird im Beltz Testmanual (1997) wie folgt beschrieben:

Personen mit ESTJ-Präferenzen benutzen analytische Beurteilung, um Dinge in den Griff zu bekommen. Gern entwickeln sie Projekte, um sie anschliessend umzusetzen. Da sie sich auf ihr analytisches Urteil verlassen, erscheinen sie analytisch, objektiv kritisch und lassen sich meist nur durch vernünftige Argumente überzeugen. Ihre Aufmerksamkeit gilt vor allem der Arbeit und nicht so sehr den Menschen, die Arbeit tun.

Sie hantieren gern mit Daten, planen Abläufe und einzelne Schritte für ein Projekt und gehen systematisch vor, um ihr Ziel termingerecht zu erreichen. Sie werden ungeduldig, wenn die Dinge unklar oder nicht effizient sind und können hart sein, wenn es die Umstände verlangen.

Ihr Weltbild und ihr eignes Leben ist geordnet.

Sie interessieren sich eher für reale Verhältnisse als für zukünftige Möglichkeiten. Das macht sie nüchtern, praktisch und realistisch, orientiert am Hier und Jetzt. Sie verlassen sich auf ihre bisherigen Erfahrungen, um Probleme zu lösen und möchten sicherstellen, das Ideen, Pläne und Entscheidungen auf solider Basis stehen.

Sie bevorzugen Aufgaben, die zu handgreiflichen, sichtbaren und konkreten Ergebnissen führen. Sie interessieren sich für Geschäftliches, für Industrie, Produktion und Konstruktion. Sie mögen Verwaltungsaufgaben, in denen sie Ziele setzen, Entscheidungen treffen und die notwendigen Anweisungen geben. Sie erledigen Dinge - das ist ihre starke Seite.

Wie alle Typen, die schnell urteilen, stehen sie in der Gefahr, voreilige Schlüsse $\mathrm{zu}$ ziehen. Sie müssen also gegensteuern und sollten besonders die Meinung derer anhören, die sich - vielleicht aufgrund ihrer Stellung - nicht sofort äussern. Das wird ihnen schwerfallen.

Möglicherweise kostet es sie Überwindung, persönliche Dinge einzubeziehen. Sie können ihre logische Seite so in den Vordergrund stellen, dass sie dabei persönliche Aspekte, die wichtig sind, nicht berücksichtigen.

Das Bild des erfolgreichen Managers, das oben gezeichnet wird, deckt sich zu weiten Teilen mit den Anforderungen, die auch in Stelleninseraten immer wieder an Führungskräfte, die im operativen Bereich tätig sind, gestellt werden:

- Schnelle Auffassungs- und Beurteilungsgabe

- Entscheidungsstark

- Systematisch, effizient, termingerecht

- Hart in der Sache

- Orientiert am Hier und Jetzt 
In der Fitnessbranche, die so sehr vom Umgang mit den Kunden, von der Atmosphäre im Center lebt, wo Kompetenz und Freundlichkeit des Personals an oberster Stelle stehen, in dieser Branche kann man sich den knallharten, analytisch-rationalen Manager kaum vorstellen. In unserer Untersuchung haben viele grosse und sehr grosse Center mitgemacht. In diesen Centern ist der Manager wohl eher hinter den Kulissen tätig und nicht noch gleichzeitig an der Rezeption und Trainingsleiter. Für diese Tätigkeiten ist das ESTJ-Profil sicher ein Vorteil. Vielleicht erscheint Personalführung auch deshalb so weit oben auf der Liste der Erfolgsfaktoren, weil sich die Manager bewusst sind, dass eben hier ein Manko besteht.

Ebenfalls fehlt das Visionäre im ESTJ-Profil. Da die Fitnessbranche sich jedoch im Stadium der Reife befindet, ist vielleicht dieses Visionäre nicht so wichtig. Eigentlich ist die Fitnessbranche ja eine relativ unkreative Branche. Abgesehen von einzelnen Trends hat sich in den letzten 5 Jahren im Vergleich zum Beispiel zur Internet-Branche kaum etwas geändert. Es geht vielmehr darum, eine solide, relativ klar umrissene Leistung für den Kunden zu vollbringen. Und dafür sind die ESTJ-Typen sicher hervorragend geeignet.

Dazu kommt, dass man auf der operativen Stufe im täglichen Geschäft gar keine Zeit hat für die Zukunft. In grossen Firmen sind deshalb operative Tätigkeiten und strategische auch getrennt, weil wer die grossen Züge sieht, in der Regel kein Flair für den so wichtigen Kleinkram hat und umgekehrt.

Das Fitnesscenter ist jedoch zu klein, als dass man operative und strategische Führung trennen könnte. Beides muss durch den Centerleiter abgedeckt werden, was zu Konflikten führen kann, wie auch URSPRUNG (1998) beschreibt:

Die Forderungen, die der Betriebsalltag an den Fitnesscenter-Manager stellt, sind hoch und bergen das Risiko, dass die konzeptionelle Arbeit vernachlässigt wird, was sich mittelfristig negativ auf den Erfolg des Unternehmens auswirkt.

Die konzeptionelle und visionäre Seite geht dem ESTJ-Typen ab. Hier sind N-Typen gefragt. Es ist deshalb nicht erstaunlich, dass auf strategischer Ebene im Führungsbereich vermehrt ENTJ-Typen angetroffen werden. Bei der bereits zitierten Untersuchung von BENTS und BLANK (1995) bei 834 Führungskräften sind die ENTJTypen mit 20\% stärker vertreten als die ESTJ-Typen mit 17\%. Die NT-Typen machen $36 \%$ aus und überflügeln die ST-Typen mit $31 \%$.

Für den Fitnessbereich ist zu sagen, dass gerade das Visionäre und das PersönlichMenschliche eine grosse Chance in den nächsten Jahren darstellt. Es wäre nicht verwunderlich, wenn sich der ENTJ-Anteil auf Kosten des ESTJ-Anteils stark erhöhen würde. Mehr noch: es wäre wünschenswert und wichtig für die Zukunft der Branche. 


\subsection{Persönlichkeit und Erfolgskennzahlen}

Im Unterkapitel 12.9 Erfolgsfaktor Effizienz (Seite 95 wurden drei Effizienzkennzahlen ermittelt:

- Mitglieder/Mitarbeiter

- $\mathrm{m}^{2} /$ Mitglied

- $\mathrm{m}^{2} /$ Mitarbeiter

Korreliert man die 4 Ausprägungen der MBTI-Persönlichkeitstypen mit diesen Effizienz-Kennzahlen, so ergeben sich keine signifikanten Zusammenhänge. Tendenziell kann man feststellen, dass die S- und T-Typen eher mehr $\mathrm{m}^{2} /$ Mitglied und eher mehr $\mathrm{m}^{2} /$ Mitarbeiter haben und in diesem Sinne erfolgversprechend arbeiten (vergleiche 12.9 Erfolgsfaktor Effizienz, Seite 95. Man stösst hier aber klar auf die Grenzen dieser Untersuchung. Einerseits aufgrund der Beschränkung auf quantitative Erhebung und andererseits auch aufgrund der relativ kleinen Stichprobenzahl.

\subsection{Zusammenfassung „Persönlichkeit $\leftarrow \rightarrow$ Erfolg“}

- Der Erfolgstyp schlechthin in unserer Untersuchung ist der ESTJ-Typ, der klassisch analytisch-rationale Manager.

- Sportstudierende sind ein gutes Rekrutierungsfeld für zukünftige Manager. Ihr ESTJ-Anteil beträgt $20 \%$.

- Das Menschlich-Soziale und Visionäre, das dem jetzigen Erfolgstypen fehlt, sind die Chancen der Branche in der näheren Zukunft. 


\section{Zusammenhänge zwischen Situation und Erfolg}

\subsection{Rangliste der situativen Erfolgsfaktoren}

Korreliert man die verschiedenen situativen Faktoren der Untersuchung mit Erfolg und erstellt anschliessend eine Rangliste aufgrund der Signifikanz, so ergibt sich folgendes Bild.

\begin{tabular}{|l|l|l|l|}
\hline Rang & Situativer Faktor & Korrelation & Signifikanz \\
\hline 1 & Kompetenz des Personals & 0.373 & 0.001 \\
\hline 2 & Freundlichkeit des Personals & 0.373 & 0.003 \\
\hline 3 & Breite des Angebots & 0.253 & 0.027 \\
\hline 4 & Sauberkeit der Nasszone & 0.249 & 0.026 \\
\hline 5 & Moderne Maschinen und Geräte & 0.235 & 0.036 \\
\hline 6 & Anzahl Parkplätze & 0.205 & 0.068 \\
\hline 7 & Erreichbarkeit mit öffentl. Verkehr & 0.194 & 0.085 \\
\hline 8 & Versch. Abonnements-Möglichkeiten & 0.830 & 0.464 \\
\hline 9 & Kollegen trainieren hier & 0.081 & 0.479 \\
\hline 10 & Zentrale Lage & 0.074 & 0.513 \\
\hline 11 & Helle, hohe Räumlichkeiten & 0.028 & 0.807 \\
\hline 12 & Kinderbetreuung & 0.002 & 0.988 \\
\hline
\end{tabular}

Tabelle 17-1 Rangliste der situativen Erfolgsfaktoren

Wirklich signifikant korrelieren die ersten fünf Faktoren mit Erfolg. Es kann also festgehalten werden: Je besser die folgenden Faktoren erfüllt werden, desto erfolgreicher ist der jeweilige Fitnesscenter-Manager:

- Kompetenz des Personals

- Freundlichkeit des Personals

- Sauberkeit der Nasszone

- Breite des Angebots

- Moderne Maschinen und Geräte

Diese Ergebnisse decken sich mit den in Unterkapitel 13.2 Erfolgsfaktoren aus Kundensicht (Seite 101) gemachten Feststellungen. Dort erstellten die Befragten eine Rangliste der für die Kunden wichtigsten Faktoren. In den ersten fünf Rängen tauchten auf:

1. Freundlichkeit des Personals

2. Kompetenz des Personals

3. Anzahl Parkplätze

4. helle, hohe Räumlichkeiten

5. Breite des Angebots 
Es zeigt sich, dass die damals für die Kunden als wichtig erachteten Kriterien sich auch direkt auf den Erfolg des Centers auswirken. An erster Stelle steht dabei wieder das Personal. Je kompetenter und je freundlicher das Personal, desto erfolgreicher ist das Center. Die beiden Signifikanzen von 0.001 und 0.003 sprechen eine deutliche Sprache.

Diese Resultate stützen die Ergebnisse des Kapitels, Zusammenhänge zwischen Führungsverhalten und Erfolg ' wo gezeigt wurde, dass der sehr erfolgreiche Manager am meisten Zeit für „Personal führen“ einsetzt (vergleiche 15.4 Führungsverhalten des sehr erfolgreichen Managers, Seite 120.

\subsection{Erfüllung der Erfolgsfaktoren durch die einzelnen Center}

Die folgende Liste zeigt die Unterschiede zwischen den erfolgreichen und den nicht erfolgreichen Centern im Bereich der Erfüllung der oben erwähnten Erfolgsfaktoren.

\begin{tabular}{|l|l|l|}
\hline Situativer Faktor & \multicolumn{2}{|l|}{ Erfüllung der Anforderung durch } \\
\hline & mässig erfolgreiche & sehr erfolgreiche \\
\hline & Fitnesscenter \\
\hline Kompetenz des Personals & 3.32 & 4.00 \\
\hline Freundlichkeit des Personals & 3.36 & 4.00 \\
\hline Breite des Angebots & 2.80 & 3.00 \\
\hline Sauberkeit der Nasszone & 3.40 & 4.00 \\
\hline Moderne Maschinen und Geräte & 3.16 & 3.67 \\
\hline Anzahl Parkplätze mit öffentl. & 2.72 & 3.33 \\
\hline $\begin{array}{l}\text { Erreichbarkeit Abonnements- } \\
\text { Verkehr }\end{array}$ & 3.36 & 3.67 \\
\hline $\begin{array}{l}\text { Versch. } \\
\text { Möglichkeiten }\end{array}$ & 2.62 & 3.67 \\
\hline Kollegen trainieren hier & 2.96 & 3.17 \\
\hline Zentrale Lage & 3.20 & 3.33 \\
\hline Helle, hohe Räumlichkeiten & 2.40 & 3.50 \\
\hline Kinderbetreuung & \multicolumn{2}{|l}{} \\
\hline
\end{tabular}

Legende

Bewertung der Erfüllung: 1 = nicht erfüllt, $2=+/$ - erfüllt, $3=$ gut erfüllt, $4=$ sehr gut erfüllt.

Tabelle 17-2 Erfüllung der Erfolgsfaktoren

Ausser beim Faktor Breite des Angebots sind die sehr erfolgreichen Center bei den wichtigsten 6 Erfolgsfaktoren jeweils über einen halben Bewertungspunkt besser als die nur mässig erfolgreichen Center. Grundsätzlich schneiden die sehr erfolgreichen Center in allen Erfolgsfaktoren besser ab als die mässig erfolgreichen.

Dies ist eine Bestätigung für die Validität der herausgearbeiteten Erfolgsfaktoren. Offensichtlich sind sie ein guter und verlässlicher Indikator für Erfolg. 
- Die wichtigsten situativen Faktoren für Erfolg sind nach ihrer Wichtigkeit sortiert:

- Kompetenz des Personals

- Freundlichkeit des Personals

- Sauberkeit der Nasszone

- Breite des Angebots

- Moderne Maschinen und Geräte

- Anzahl Parkplätze

- Diese Erfolgsfaktoren werden durch sehr erfolgreiche Center markant besser erfüllt als durch die nur mässig erfolgreichen Center. 


\section{Zusammenhänge zwischen Persönlichkeit und Situation}

\subsection{Erfüllung der situativen Erfolgsfaktoren durch die einzelnen Typen}

Generell kann man kaum tendenzielle oder sogar signifikante Zusammenhänge zwischen den einzelnen Persönlichkeitstypen und den situativen Faktoren zeigen. Die Auflistung der jeweils besten und schlechtesten Erfüllung der verschiedenen situativen Erfolgsfaktoren durch die einzelnen Ausprägungen der MBTI-Typen zeigt jedoch ein paar interessante Resultate.

\begin{tabular}{|l|l|l|}
\hline Situativer Faktor nach Wichtigkeit & $\begin{array}{l}\text { Beste } \\
\text { Erfüllung }\end{array}$ & $\begin{array}{l}\text { Schlechteste } \\
\text { Erfüllung }\end{array}$ \\
\hline Kompetenz des Personals & $3.65(\mathrm{P})$ & $3.35(\mathrm{I})$ \\
\hline Freundlichkeit des Personals & $3.61(\mathrm{~S})$ & $3.35(\mathrm{I})$ \\
\hline Breite des Angebots & $3.14(\mathrm{~S})$ & $2.97(\mathrm{~F})$ \\
\hline Sauberkeit der Nasszone & $3.61(\mathrm{~S})$ & $3.35(\mathrm{I})$ \\
\hline Moderne Maschinen und Geräte & $3.38(\mathrm{~J})$ & $3.13(\mathrm{P})$ \\
\hline Anzahl Parkplätze & $3.06(\mathrm{P}, \mathrm{T})$ & $2.75(\mathrm{I})$ \\
\hline Erreichbarkeit mit öffentl. Verkehr & $3.30(\mathrm{I})$ & $2.97(\mathrm{~S})$ \\
\hline Versch. Abonnements-Möglichkeiten & $3.31(\mathrm{~F})$ & $3.06(\mathrm{P})$ \\
\hline Kollegen trainieren hier & $2.98(\mathrm{~N})$ & $2.50(\mathrm{~S})$ \\
\hline Zentrale Lage & $3.40(\mathrm{I})$ & $2.75(\mathrm{~S})$ \\
\hline Helle, hohe Räumlichkeiten & $3.39(\mathrm{~S})$ & $3.13(\mathrm{P})$ \\
\hline Kinderbetreuung & $2.73(\mathrm{~N})$ & $2.28(\mathrm{~S})$ \\
\hline
\end{tabular}

Legende

Bewertung der Erfüllung: 1 = nicht erfüllt, $2=+/$ - erfüllt, $3=$ gut erfüllt, $4=$ sehr gut erfüllt.

\section{Tabelle 18-1 Erfüllung der sit. Erfolgsfaktoren nach Typen}

Unter den Extremen sind der S-Typ (4+, 4-), der I-Typ (2+, 4-) und der P-Typ (2+, 3-) am meisten vertreten. N-, T-, F- und J-Typ kommen je $1-2 \mathrm{x}$ vor, der E-Typ taucht nicht auf.

Der S-Typ ist auffällig gut positioniert. Er erfüllt von den 6 wichtigsten Erfolgsfaktoren deren 3 am besten. Der I-Typ hingegen erfüllt von den 6 wichtigsten Faktoren 3 am schlechtesten. Diese Beobachtungen bestätigen die Aussagen in Unterkapitel 16 Zusammenhang zwischen Persönlichkeit und Erfolg (Seite 122). Dort wurde die S- nach der T-Komponente als die erfolgversprechendste Konstellation bezeichnet. Aufgrund der obigen Erkenntnisse müsste man den S-Typ sogar noch vor den T-Typen stellen.

Es wurde in Unterkapitel 16 auch erwähnt, dass E-Typen grundsätzlich erfolgreicher als I-Typen sind. Die Häufung der schlechtesten Erfüllung bei den wichtigsten Erfolgsfaktoren durch I-Typen dürfte aber wahrscheinlich eher zufällig sein. Sie 
bestätigt jedoch den sich abzeichnenden Trend von eher erfolgversprechenden oder eben weniger erfolgversprechenden Persönlichkeitsmerkmalen.

18.2 Zusammenfassung „Persönlichkeit $\leftrightarrow \rightarrow$ Situation“

- Grundsätzlich sind keine Signifikanzen und wenig Tendenzen von Zusammenhängen zwischen Persönlichkeit und Situation feststellbar.

- Zwei erwähnenswerte Beobachtungen: Der S-Typ erfüllt drei der sechs wichtigsten Erfolgsfaktoren am besten. Der I-Typ hingegen erfüllt drei der sechs wichtigsten Erfolgsfaktoren am schlechtesten. 


\section{Unterschiede Stadt-Land und Gross-Klein}

In den vorherigen Kapiteln wurden die Auswertung jeweils für die Gesamtheit aller Center vorgenommen. Verschiedene Thesen sind bei dieser Auswertung aufgetaucht:

Unterschiede Stadt - Land

- Center in der Stadt sind grösser und haben mehr Kunden als Center auf dem Land.

- Die Persönlichkeit des Stadt-Managers unterschiedet sich von der Persönlichkeit des Land-Managers.

- Der Konkurrenzdruck ist in der Stadt höher als auf dem Land.

- In einem Stadtcenter hat es mehr Teilzeitangestellte als in einem Landcenter.

- In der Stadt sind die Führungstätigkeiten Marketing und Personal führen wichtiger als auf dem Land.

Unterschiede Gross - Klein

- Grosse Center haben im Verhältnis mehr Kunden als die kleinen Center.

- Die Persönlichkeit des Managers eines kleinen Centers unterschiedet sich von der Persönlichkeit des Managers eines grossen Centers.

- Der Konkurrenzdruck ist für die kleinen Center grösser als für die grossen.

- In einem grossen Center hat es mehr Teilzeitangestellte als in einem kleinen Center.

- Im grossen Center sind die Führungstätigkeiten Marketing und Personal führen wichtiger als im kleinen Center.

Diesen Thesen wird in diesem Kapitel nachgegangen.

\subsection{Unterschiede Stadt - Land}

Die Unterteilung in Center auf dem Land und Center in der Stadt wurde wie folgt vorgenommen.

- Ortschaft mit weniger als 15'000 Einwohnern: Land

- Ortschaft mit mehr als 15'000 Einwohnern: Stadt

\subsubsection{Verteilung}

Die Unterteilung nach obigem Kriterium ergibt folgende Verteilung:

\begin{tabular}{|l|l|l|}
\hline Centertyp & Total effektiv & Total in \% \\
\hline Land & 45 & 57 \\
\hline Stadt & 34 & 43 \\
\hline
\end{tabular}

Tabelle 19-1 Verteilung Stadt-Land 
Über die Hälfte der Center befinden sich auf dem Land. Diese Verteilung spiegelt die Siedelungsstruktur wieder. Die Schweiz hat abgesehen von Zürich, Bern und Genf und Basel wenig ganz grosse Ballungszentren, sondern ist - zumindest was das Mittelland anbetrifft - fast durchgehend und mit vielen kleineren Dörfern und Städten besiedelt.

\subsubsection{Grösse}

Werden die einzelnen Center nach Grösse sortiert, ergibt sich folgdendes Bild:

\begin{tabular}{|l|l|l|l|}
\hline Centerfläche & Verteilung CH in \% & Verteilung Stadt in \% & Verteilung Land in \% \\
\hline $0-399 \mathrm{~m}^{2}$ & 20.5 & 12.5 & 27.3 \\
\hline $400-799 \mathrm{~m}^{2}$ & 34.6 & 34.4 & 36.4 \\
\hline $800-1199 \mathrm{~m}^{2}$ & 21.8 & 12.5 & 27.3 \\
\hline$>1200 \mathrm{~m}^{2}$ & 23.1 & 40.6 & 9.0 \\
\hline
\end{tabular}

Tabelle 19-2 Flächenverteilung Stadt-Land

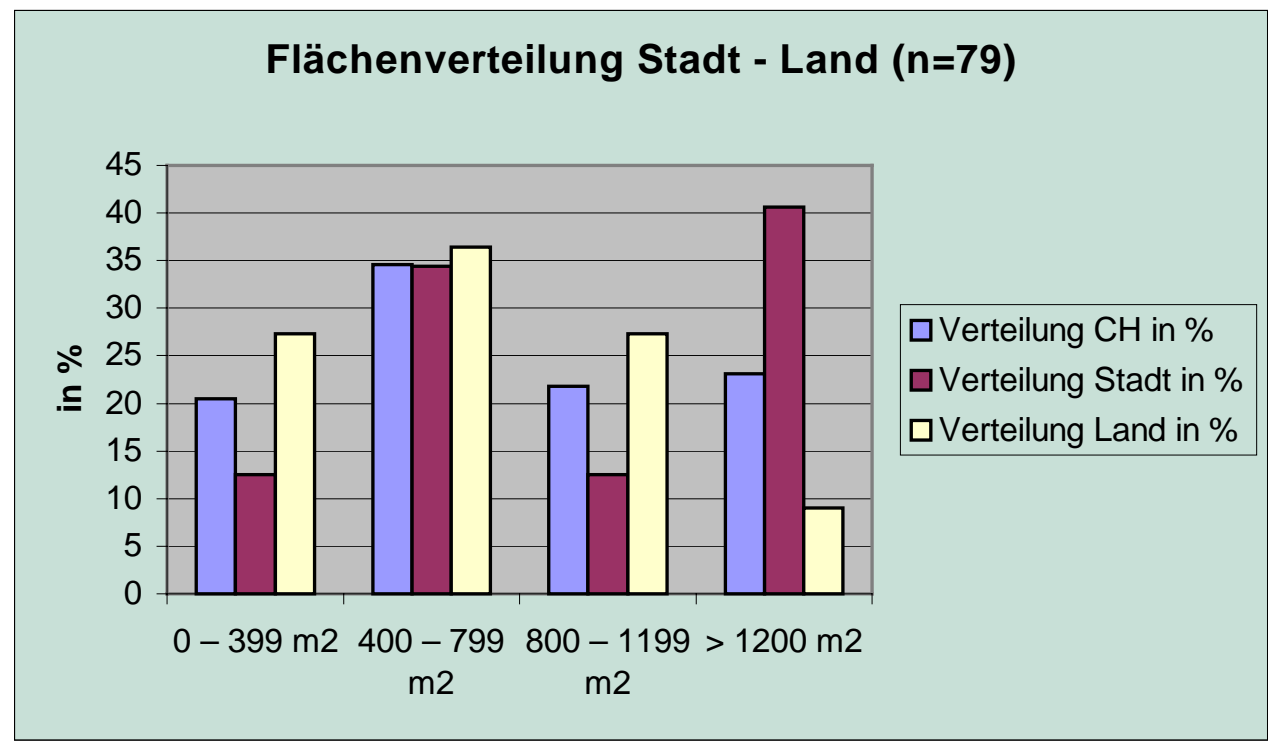

\section{Abbildung 19-1 Flächenverteilung Stadt - Land}

Bereits die graphische Darstellung zeigt, dass sich die These bestätigt, dass tendenziell in der Stadt die grösseren Center und auf dem Land die kleineren Center zu finden sind. Der Korrelationstest nach Mann-Whitney ergibt keine klare Signifikanz $(\mathrm{u}=525$ und $\mathrm{p}$ = 0.059), aber immerhin eine starke Tendenz zur Signifikanz.

Es lässt sich also festhalten, dass die Center auf dem Land flächenmässig tendenziell signifikant kleiner sind als die Center in der Stadt. Die Durchschnittsfläche aller Stadtcenter beträgt $925 \mathrm{~m}^{2}$, diejenige der Landcenter $672 \mathrm{~m}^{2}$. 


\subsubsection{Anzahl Kunden}

Werden die einzelnen Center nach der Anzahl Kunden sortiert, ergibt sich folgendes Bild:

\begin{tabular}{|l|l|l|l|}
\hline Anzahl Kunden & Verteilung CH in \% & Verteilung Stadt in \% & Verteilung Land in \% \\
\hline $0-299$ & 20.3 & 33.3 & 17.2 \\
\hline $300-599$ & 20.0 & 12.5 & 25.7 \\
\hline $600-899$ & 20.0 & 0 & 34.3 \\
\hline $900-1499$ & 15.0 & 20.8 & 11.4 \\
\hline$>1500$ & 21.7 & 33.4 & 11.4 \\
\hline
\end{tabular}

Tabelle 19-3 Kundenverteilung Stadt-Land

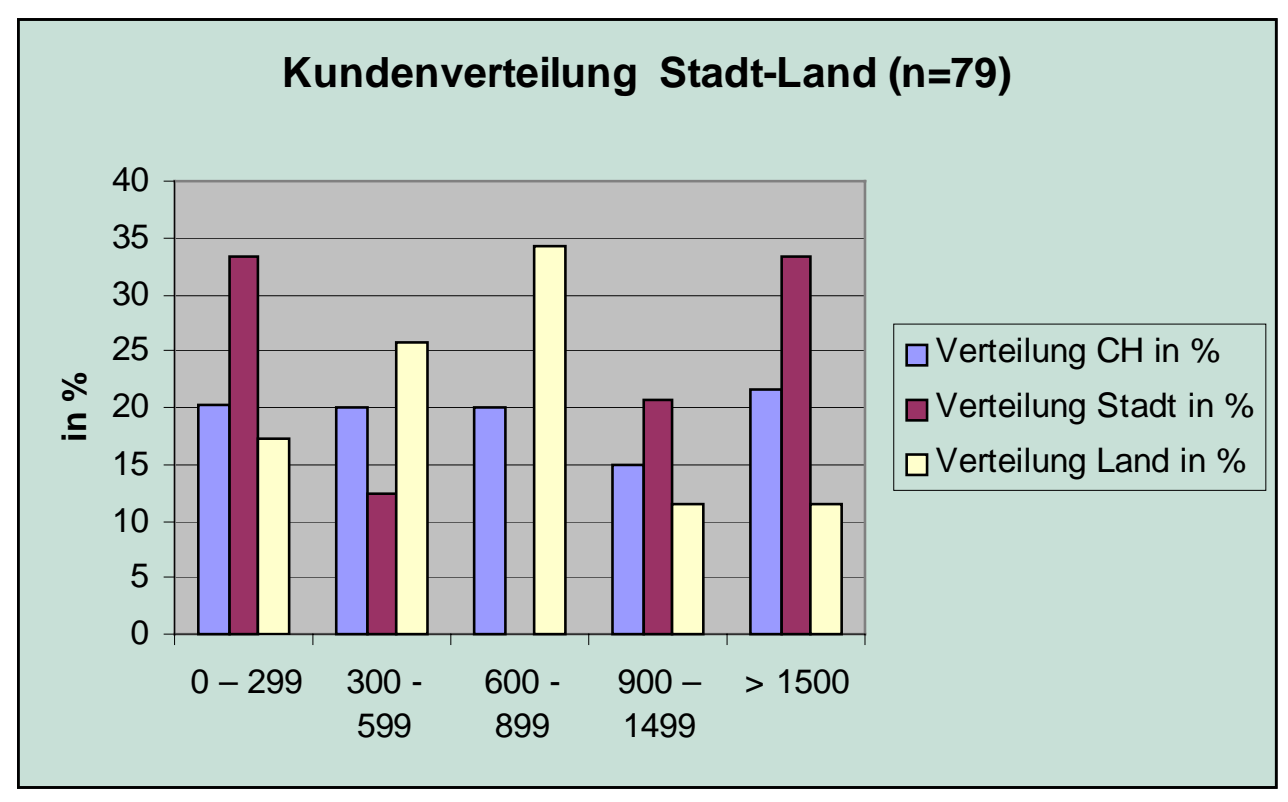

Abbildung 19-2 Kundenverteilung Stadt - Land

Die graphische Darstellung zeigt, dass es in der Stadt viel sehr kleine (unter 300 Mitglieder) und viel sehr grosse Center (über 1500 Kunden) hat. Das Land hat dafür sehr viele mittelgrosse Center (zwischen 300 und 900 Mitgliedern). In der Korrelationsanalyse nach Mann-Withney wird ebenfalls bestätigt, dass die These, in der Stadt hätten die Center mehr Kunden, nicht richtig ist $(\mathrm{u}=378.5, \mathrm{p}=0.52)$.

In der Verbindung mit den Resultaten der Fläche kann also formuliert werden, dass in der Stadt die grösseren Center sind als auf dem Land, diese Center aber nicht mehr Kunden haben als auf dem Land. Dies bedeutet, dass dem Stadtkunden mehr Platz (1.07 $\mathrm{m}^{2}$ ) zur Verfügung steht als dem Landkunden $\left(0.92 \mathrm{~m}^{2}\right)$. 


\subsubsection{Manager-Persönlichkeit}

Die Untersuchung der Persönlichkeitsverteilung zwischen Stadt- und Land-CenterManagern ergibt, dass es keine signifikanten Zusammenhänge zwischen den Persönlichkeitstypen gibt. Insbesondere die These, in der Stadt würden eher die sensitivrationalen ST-Typen, die klassischen Manager-Typen, vorherrschen, kann nicht bestätig werden (S-N: $\mathrm{p}=0.63$, T-F: $\mathrm{p}=0.76$ ).

Auch die Vorstellung, dass eher extrovertierte Typen in der Stadt zu finden sind und introvertierte das Land vorziehen, kann nicht bestätigt werden (E-I: $p=0.53$ ).

Am ehesten kann man sagen, dass es auf dem Land mehr J- als P-Typen hat (Stadt: $18 \mathrm{~J}$, 16 P; Land $30 \mathrm{~J}, 15 \mathrm{P})$, von Signifikanz ist man aber weit entfernt $(\mathrm{p}=0.22)$.

Man kann also folgern, dass es grundsätzlich keine sgnifikanten Unterschiede in den Persönlichkeiten von Stadt- und Landmanagern gibt.

\subsubsection{Konkurrenzdruck}

Der Konkurrenzdruck wird durch die Manager wie folgt eingeschätzt:

\begin{tabular}{|l|l|l|l|}
\hline Konkurrenzdruck & Verteilung CH in \% & Verteilung Stadt in \% & Verteilung Land in \% \\
\hline $1=$ klein & 12.7 & 18.2 & 5.8 \\
\hline $2=$ erträglich & 43.0 & 45.5 & 46.2 \\
\hline $3=$ gross & 30.4 & 22.7 & 38.3 \\
\hline $4=$ sehr gross & 13.9 & 13.6 & 14.7 \\
\hline
\end{tabular}

Tabelle 19-4 Verteilung des Konkurrenzdruckes Stadt - Land

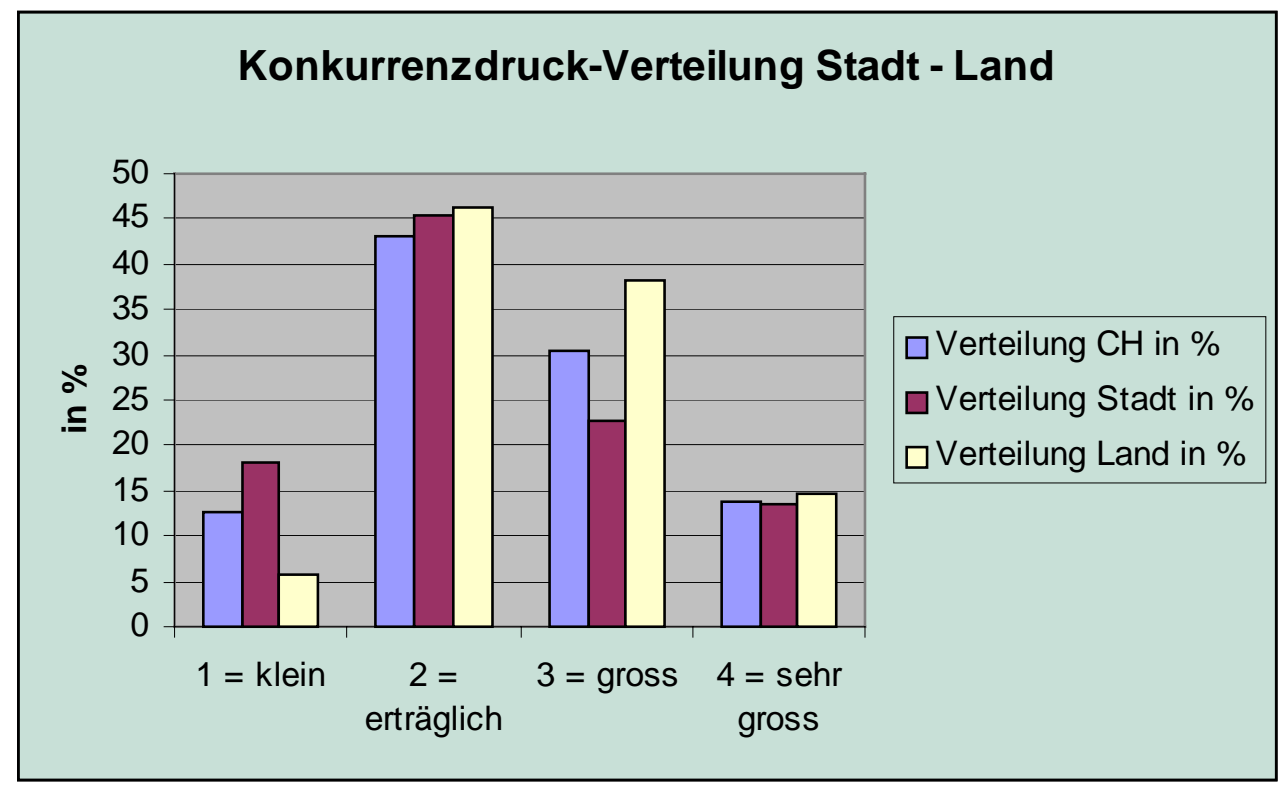




\section{Abbildung 19-3 Verteilung des Konkurrenzdruckes Stadt - Land}

Der Konkurrenzdruck wird von mehr Stadtcentern als klein angesehen als dies bei Landcentern der Fall ist. Bei Landcentern sind auch mehr Manager der Meinung, der Konkurrenzdruck sei gross, als dies bei Stadtcenter der Fall ist. Signifikant sind die Unterschiede nicht $(\mathrm{p}=0.12)$, eine Tendenz zur Signifikanz kann man aber immerhin postulieren.

Ein mögliche Interpretation der Ergebnisse wurde bereits im Unterkapitel 9.4 (Validitätsprüfung anhand der Konkurrenzsituation Seite 63) vorgestellt. Durch den effektiv höheren Konkurrenzdruck wegen der grösseren Centerdichte in der Stadt, müssen sich die einzelnen Center in besonderen Nischen gegenüber ihrer Konkurrenz profilieren. Diese Positionierung bewirkt natürlich wieder eine Abnahme der direkten Konkurrenten.

\subsubsection{Personal}

Bei der Gegenüberstellung der Stadt- und Landcenter im Bereich des Personals ergeben sich folgende Resultate:

\section{In den Stadt-Centern hat es}

Mehr Vollzeitangestellte in der Stadt: Mehr Teilzeitangestellte in der Stadt:

Mehr Angestellte in der Stadt:

$$
\begin{aligned}
& p=0.001 \\
& p=0.087 \\
& p=0.044
\end{aligned}
$$

\section{Tabelle 19-5 Personalvergleich Stadt - Land}

In allen drei Teilbereichen lässt sich feststellen, dass in der Stadt signifikant mehr Personal als auf dem Land vorhanden ist. Im Bereich der Vollzeitangestellten hat es sogar höchst signifikant mehr Personal in der Stadt als auf dem Land. Offensichtlich braucht es in der Stadt mehr Personal, um den Anforderungen der Kunden und dem grösseren effektiven Konkurrenzdruck standzuhalten.

Zusätzlich haben wir im Unterkapitel 19.1.2 Grösse Seite 135 und 19.1.3 Anzahl Kunden Seite 136 festgestellt, dass auch die Fläche tendenziell in der Stadt grösser ist, die Anzahl der Kunden jedoch nicht.

Wir können daraus folgern, dass die Stadt-Center die höheren fixen Kosten haben und deshalb wahrscheinlich auch einen höheren Abonnementspreis verlangen müssen, um gleich rentabel zu sein wie die Landcenter. Diese Fragen lassen sich aber mit der bestehenden Untersuchungsanlage nicht beantworten, sondern müssten in weiterführenden Studien angegangen werden. 


\subsubsection{Führungsverhalten}

Im Fragebogen-Teil Führungsverhalten mussten die Manager darüber Auskunft geben, welche Führungstätigkeiten sie im Hinblick auf Erfolg als besonders wichtig erachteten und wie viel Zeit sie effektiv für die einzelnen Tätigkeiten aufwendeten.

Wichtiger für Erfolg in der Stadt

Personal führen

$$
\begin{aligned}
& p=0.82 \\
& p=0.07 \\
& p=0.48 \\
& p=0.94
\end{aligned}
$$

\section{Tabelle 19-6 Wichtigkeitseinschätzung Führungsverhalten Stadt - Land}

Die These war, dass Marketing und Akquisition in der Stadt als wichtiger für Erfolg eingeschätzt wird als auf dem Land. Die These muss aber verworfen werden. Erstaunlicherweise herrscht aber in der Stadt mit Tendenz zur Signifikanz öfter die Meinung vor, Papierarbeit und Administration sei wichtig für Erfolg.

Offensichtlich gehen die Stadtmanager davon aus, dass dies für ihre Kunden sehr wichtig ist. Dem Landkunden hingegen kommt es eher auf das Angebot als auf die perfekte Abo-Administration an.

Neben der Einschätzung der Wichtigkeit für den Erfolg wurde die effektiv verwendete Zeit für die betreffenden Führungstätigkeiten erhoben. Hier ergibt sich folgendes Bild in der Stadt-Land-Gegenüberstellung:

Mehr Zeit für die entsprechende Tätigkeit aufgewendet in der Stadt

Personal führen

Administration, Papierarbeit

Akquisition, Marketing

Repräsentation, soziale Verpflichtungen

$$
\begin{aligned}
& p=0.785 \\
& p=0.782 \\
& p=0.767 \\
& p=0.513
\end{aligned}
$$

Tabelle 19-7 Vergleich Führungstätigkeiten Stadt - Land

Es zeigen sich keinerlei Signifikanzen. Konsequenterweise müsste der Stadtmanager ja mehr Zeit für Administration aufwenden. Dies ist aber nicht der Fall.

Man kann also zusammenfassend folgern, dass sich die Einschätzung der Wichtigkeit von Führungstätigkeiten nur im Bereich Administration/Papierarbeit von Stadt zu Landmanager unterscheiden. Im Bereich der effektiven Führungsarbeit lassen sich keine signifikanten Unterschiede zwischen Stadt und Land beobachten. 


\subsubsection{Erfolg}

Sind Center in der Stadt erfolgreicher als Center auf dem Land?

Die Korrelationsanalyse nach Mann-Whitney ergibt ein Resultat von $\mathrm{u}=694$ und $\mathrm{p}=$ 0.584, womit die obige Frage mit nein beantwortet werden muss.

Es gibt keine signifikanten Unterschiede in der persönlichen Erfolgseinschätzung zwischen Stadt- und Land-Managern.

\subsubsection{Zusammenfassung}

Zusammenfassend lassen sich folgende Thesen zu den beiden Vergleichsgruppen Center auf dem Land und Center in der Stadt formulieren:

- Die Center in der Stadt sind flächenmässig tendenziell signifikant grösser als die Center auf dem Land. Die Durchschnittsfläche aller Stadtcenter beträgt $925 \mathrm{~m}^{2}$, diejenige der Landcenter $672 \mathrm{~m}^{2}$. Die Stadt-Center haben jedoch nicht mehr Kunden als die Land-Center. Dies bedeutet, dass dem Stadtkunden mehr Platz $\left(1.07 \mathrm{~m}^{2}\right)$ zur Verfügung steht als dem Landkunden $\left(0.92 \mathrm{~m}^{2}\right)$.

- Im Bereich der Persönlichkeit gibt es zwischen den Persönlichkeiten von Stadt- und Landmanagern keine signifikanten Unterschiede.

- Der Konkurrenzdruck wird von den Stadtcentern tendenziell signifikant kleiner eingeschätzt als durch die Landcentern, obwohl die Fitnesscenter-Dichte in der Stadt viel grösser ist als auf dem Land. Eine erfolgreiche Nischenpolitik ist offenbar dafür verantwortlich.

- In den Centern der Stadt ist signifikant mehr Personal vorhanden als auf dem Land. Im Bereich der Vollzeitangestellten hat es sogar höchst signifikant mehr Personal. Offensichtlich braucht es in der Stadt mehr Personal, um den Anforderungen der Kunden und dem grösseren effektiven Konkurrenzdruck standzuhalten.

- In der Einschätzung der Wichtigkeit von Führungstätigkeiten unterscheiden sich die Stadt- und Landmanager nur im Bereich von Administration/Papierarbeit. Im Bereich der effektiven Führungsarbeit lassen sich keine signifikanten Unterschiede zwischen Stadt und Land beobachten.

- Es gibt keine signifikanten Unterschiede in der persönlichen Erfolgseinschätzung zwischen Stadt- und Land-Managern. 


\subsection{Unterschiede Gross - Klein}

Die Unterteilung in grosse und kleine Center wurde wie folgt vorgenommen.

- Center mit weniger als 500 Mitgliedern: klein

- Center mit 500 - 999 Mitgliedern: mittel

- Center mit mehr als 1000 Mitgliedern: $\quad$ gross

\subsubsection{Verteilung}

Die Unterteilung nach obigem Kriterium ergibt folgende Verteilung:

\begin{tabular}{|l|l|l|}
\hline Centertyp & Total effektiv & Total in \% \\
\hline Klein & 21 & 35.0 \\
\hline Mittel & 17 & 28.3 \\
\hline Gross & 22 & 36.7 \\
\hline
\end{tabular}

\section{Tabelle 19-8 Verteilung Gross - Klein}

Es ergeben sich drei beinahe gleich grosse Gruppen. Für die weiteren Betrachtungen werden nur mehr die beiden Gruppen Klein und Gross miteinander verglichen.

\subsubsection{Manager-Persönlichkeit}

Wie schon beim Vergleich Stadt - Land besteht auch hier die These, dass grosse Center eher von ST-Managern geführt werden und dass extravertierte Typen ebenfalls eher vorherrschen. Die Korrelationsanalyse ergibt folgende Ergebnisse:

In den grossen Centern hat es

Mehr E als I-Typen: $\quad \mathrm{p}=0.234$

Mehr S als N-Typen: $\quad \mathrm{p}=0.177$

Mehr T als F-Typen: $\quad \mathrm{p}=0.612$

Mehr J als P-Typen: $\quad \mathrm{p}=0.908$

\section{Tabelle 19-9 Typenverteilung Gross - Klein}

Die Auswertung zeigt, dass sich keine signifikanten Zusammenhänge nachweisen lassen. Es hat zwar leicht mehr E-Typen in den grossen Centern als in den kleinen, aber von Signifikanz kann nicht die Rede sein.

Die grössten Unterschiede im Bereich der Persönlichkeit ergeben sich im Bereich der Ausprägung S-N. Tendenziell sind in grossen Centern die N-Typen übervertreten. Dies deckt sich mit den Bemerkungen im Unterkapitel 16.4 (Der Erfolgstyp ESTJ. Seite 124, wo postuliert wird, dass der momentane Erfolgsytp ESTJ durch den neuen Erfolgstyp ENTJ abgelöst werden wird. Dies deckt sich auch mit den Ergebnissen von 
BENTS und BLANK (1995), die im mittleren und höheren Management eher ENTJTypen, im unteren Management eher ESTJ-Typen fanden und entspricht den Erwartungen der MBTI-Typenpsychologie. Der sensitiv aufnehmende Manager ist bei zunehmender Komplexität überfordert. Je grösser der Betrieb wird, desto wichtiger ist eine intuitive Wahrnehmung der Gesamtsituation.

Zusammenfassend kann man sagen, dass eher mehr E- als I-Typen und etwas mehr Nals S-Typen in den grossen Centern vorkommen, grundsätzlich aber im Bereich der Persönlichkeitstypen keine signifikanten Unterschiede zwischen Gross und Klein bestehen.

\subsubsection{Konkurrenzdruck}

Der Konkurrenzdruck wird durch die Manager wie folgt eingeschätzt:

\begin{tabular}{|l|l|l|l|}
\hline Konkurrenzdruck & Verteilung CH in \% & Verteilung Gross in \% & Verteilung Klein in \% \\
\hline $1=$ klein & 12.7 & 19.0 & 9.1 \\
\hline $2=$ erträglich & 43.0 & 38.2 & 40.9 \\
\hline $3=$ gross & 30.4 & 33.3 & 27.3 \\
\hline $4=$ sehr gross & 13.9 & 9.5 & 22.7 \\
\hline
\end{tabular}

Tabelle 19-10 Verteilung des Konkurrenzdruckes Gross - Klein

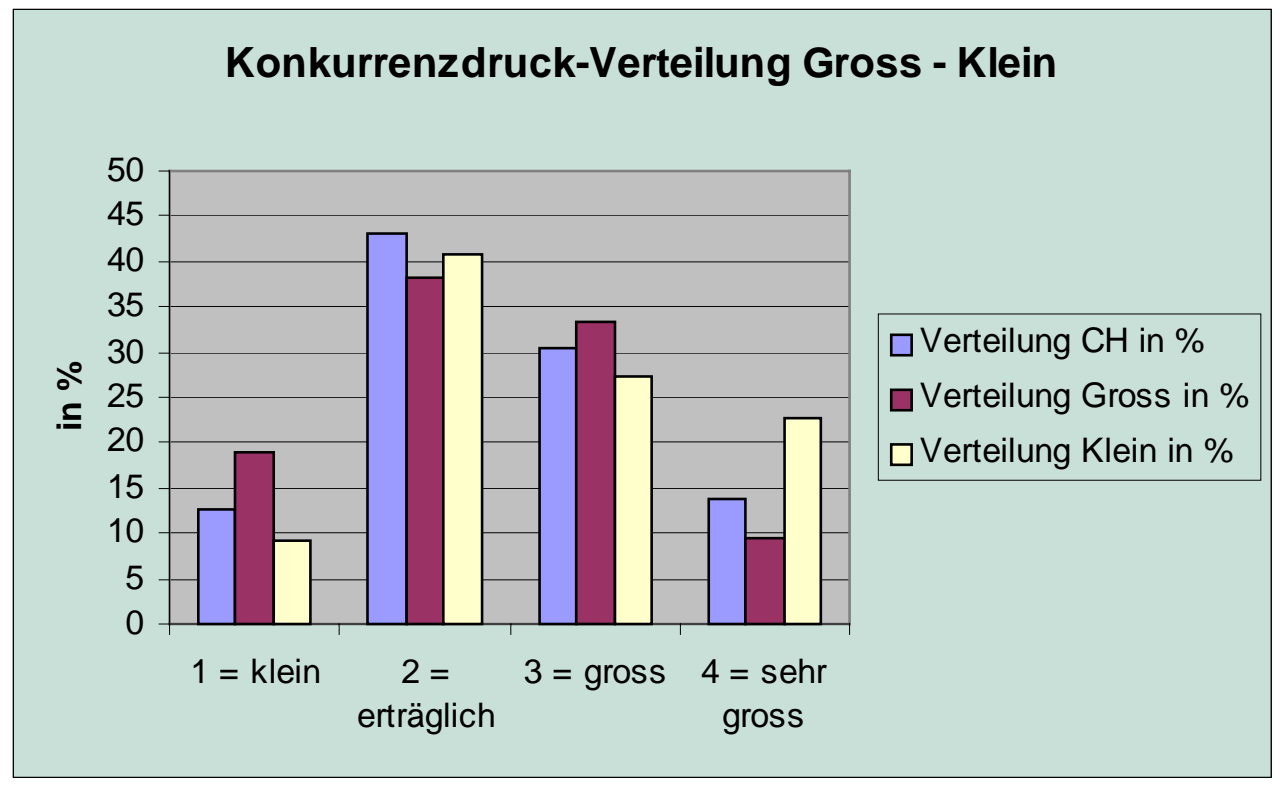

\section{Abbildung 19-4 Verteilung des Konkurrenzdruckes Gross - Klein}

Die These war, dass kleinere Center tendenziell dem grösseren Konkurrenzdruck ausgesetzt sind als grosse Center. Gesamthaft lässt sich dies nicht zeigen $(p=0.33)$. Aus der Grafik ist aber immerhin ersichtlich, dass die kleinen Center in der Gruppe mit sehr grossem Konkurrenzdruck übervertreten sind. 
Zusammenfassend kann man sagen, dass es keine wesentlichen Unterschiede im Bereich des Konkurrenzdruckes zwischen grossen und kleinen Centern gibt.

\subsubsection{Personal}

Bei der Gegenüberstellung der grossen und kleinen Center im Bereich des Personals ergeben sich folgende Resultate:

In den grossen Centern hat es

Mehr Vollzeitangestellte:

$$
\begin{aligned}
& p=0.011 \\
& p=0.003 \\
& p=0.004
\end{aligned}
$$$$
\text { Mehr Teilzeitangestellte: }
$$

\section{Tabelle 19-11 Personalvergleich Gross - Klein}

Ähnlich wie schon beim Vergleich der Stadt- und Landcenter lässt sich hier feststellen, dass in den grossen Centern signifikant mehr Personal als in den kleinen Centern vorhanden ist. Sowohl im Bereich der Teilzeitangestellten wie auch der hochgerechneten $100 \%$-Stellen hat es sogar höchst signifikant mehr Personal in den grossen Centern als in den kleinen Centern. Offensichtlich braucht es in den grossen Centern mehr Personal, um mit einem Plus an Betreuung der Anonymität, die ein grosses Center mit sich bringt, zu begegnen.

Auch hier können wir folgern, dass die grossen Center mit grösserer Fläche und mehr Personal die höheren fixen Kosten haben als die kleineren Center und deshalb wahrscheinlich auch einen höheren Abonnementspreis verlangen müssen, um gleich rentabel zu sein wie die kleinen Center. Die Höhe des Abonnementspreises wurde in dieser Studie nicht erhoben, womit auf diese Frage keine Antwort gegeben werden kann.

\subsubsection{Führungsverhalten}

Auch im Bereich des Führungsverhaltens wurde verglichen, ob sich die beiden Gruppen grosse und kleine Center signifikant unterscheiden. Die Resultate sind die folgenden:

In den grossen Centern werden folgende Führungstätigkeiten eher als wichtig eingeschätzt:

Personal führen

Administration, Papierarbeit

$\mathrm{p}=0.732$

Akquisition, Marketing

$\mathrm{p}=0.062$

Repräsentation, soziale Verpflichtungen

$\mathrm{p}=0.481$

$\mathrm{p}=0.936$

Tabelle 19-12 Wichtigkeitseinschätzung Führungsverhalten Gross - Klein 
Die These war, dass Marketing und Akquisition in den grossen Centern als wichtiger für Erfolg eingeschätzt wird als in den kleinen Centern, da die kleinen eher über Atmosphäre und Mund-zu Mund-Propaganda neue Kunden ansprechen, die grossen Center hingegen auf ein gutes Marketing angewiesen sind. Die These muss aber verworfen werden. Erstaunlicherweise herrscht aber wie schon beim Vergleich Stadt Land auch hier bei den grossen Centern mit Tendenz zur Signifikanz öfter die Meinung vor, Papierarbeit und Administration sei wichtig für Erfolg.

Diese Einschätzung leuchtet ein: je grösser das Center ist und je mehr Kunden betreut werden (müssen), desto wichtiger ist eine gut organisierte Administration. Oder anders formuliert: in einem kleinen Center mit wenig Kunden kann schlechte Administration durch den engen Kontakt mit den Kunden wettgemacht werden; bei den grossen Centern ist mit schlechter Administration das Chaos programmiert.

Neben der Einschätzung der Wichtigkeit für den Erfolg wurde auch die effektiv verwendete Zeit für die betreffenden Führungstätigkeiten erhoben. Hier ergibt sich folgendes Bild:

Mehr Zeit für die entsprechende Tätigkeit aufgewendet in den grossen Centern

Personal führen

Administration, Papierarbeit

$\mathrm{p}=0.001$

Akquisition, Marketing

$\mathrm{p}=0.897$

Repräsentation, soziale Verpflichtungen $\quad \mathrm{p}=0.369$

Tabelle 19-13 Vergleich Führungsverhalten Gross - Klein

Man würde erwarten, dass in grossen Centern der Wichtigkeit wegen mehr Zeit in Administration investiert wird. Dies ist aber nicht der Fall. Es mag daran liegen, dass in grossen Centern die Administration durch ein eigentliches Sekretariat erledigt wird und nicht mehr durch den Centermanager selber.

Höchst signifikant mehr Zeit wird aber durch den Manager grosser Center für Personalführung eingesetzt. Personalführung ist effektiv Chefsache und kann nicht delegiert werden. Bei mehr Personal in den grossen Centern war zu erwarten, dass mehr Zeit für Personalführung aufgewendet wird. Die vorliegende Deutlichkeit war hingegen nicht erwartet worden.

Man kann also zusammenfassend folgern, dass sich die Einschätzung der Wichtigkeit von Führungstätigkeiten nur im Bereich Administration/Papierarbeit von Managern grosser und kleiner Center unterscheiden. Im Bereich der effektiven Führungsarbeit wendet der Manager grosser Center höchst signifikant mehr Zeit auf für Personalführung als der Manager kleiner Center. 


\subsubsection{Erfolg}

Sind die grossen Center erfolgreicher als die kleinen Center?

Die Korrelationsanalyse nach Mann-Whitney ergibt ein Resultat von $\mathrm{u}=158$ und $\mathrm{p}=$ 0.075. Es kann also eine Tendenz zur Signifikanz gezeigt werden.

Die Manager der grösseren Center schätzen ihren Erfolg tendeziell signifikant höher ein als die Manager von kleinen Centern.

\subsubsection{Zusammenfassung}

Die Unterschiede zwischen den beiden Gruppen Grosse Center und Kleine Center lassen sich folgendermassen zusammenfassen:

- In den grossen Centern kommen eher mehr E- als I-Typen und etwas mehr N- als STypen in den grossen Centern vor. Grundsätzlich sind aber keine signifikanten Persönlichkeitsunterschiede zwischen den beiden Gruppen Gross und Klein nachzuweisen.

- Es gibt keine wesentlichen Unterschiede im Bereich des Konkurrenzdruckes zwischen grossen und kleinen Centern.

- Die grossen Center mit grösserer Fläche haben mehr Personal als die kleineren Center. Aufgrund der höheren fixen Kosten müssen sie deshalb wahrscheinlich auch einen höheren Abonnementspreis verlangen, um gleich rentabel zu sein wie die kleinen Center. Die Höhe des Abonnementspreises wurde in dieser Studie nicht erhoben, womit auf diese Frage keine Antwort gegeben werden kann.

- Die Einschätzung der Wichtigkeit von Führungstätigkeiten durch Manager von kleinen und grossen Center unterscheidet sich nur im Bereich Administration/Papierarbeit. Im Bereich der effektiven Führungsarbeit wendet der Manager grosser Center höchst signifikant mehr Zeit auf für Personalführung als der Manager kleiner Center.

- Die Manager der grösseren Center schätzen ihren Erfolg tendeziell signifikant höher ein als die Manager von kleinen Centern. 


\section{Beitrag zum Stand der Forschung}

\subsection{Erkenntnisse über die Wachstumsfaktoren der Branche}

In der Einleitung wurden aus der Literatur die drei Faktoren

- Entwicklung eines Fitnessbewusstseins

- Fitness als Mittel der Sinn- und Identitätsfindung

- Stilisierung des perfekten Körpers

als Gründe für das Wachstum der Fitnessbranche bezeichnet.

Aufgrund der vorliegenden Ergebnisse können folgende Ergänzungen und Berichtigungen angebracht werden:

Es lässt sich feststellen, dass weiterhin ein Fitnessbewusstsein in der Bevölkerung besteht. Es ist aber nicht mehr Fitness per se oder eine maximale Fitness gefragt, sondern eine zweckorientierte, im Sinne von Aufwand und Ertrag optimierte und stark individualisierte Fitness.

Je nach Kunde stehen ganz andere Gründe für Fitnesstraining im Vordergrund. Für den einen sind dies z.B. Ausgleich, Prävention von muskulären Dysbalancen des sitzenden Alltags und zugleich Herz-Kreislauftraining, um belastbarer $\mathrm{zu}$ werden und sich schneller erholen zu können. Für die anderen ist es eine Möglichkeit des Kontaktes mit der Aussenwelt, Zeit haben für sich selbst und ein Beitrag zur Selbstverwirklichung.

Jeder Kunde unterscheidet sich auch wieder darin, wieviel Aufwand er treiben will oder kann, um das persönliche Ziel zu erreichen. Ein Selbständiger kann sich vielleicht einmal in der Woche an immer wieder ändernden Zeiten und Tagen Zeit nehmen, ein Arbeitsloser trainiert vielleicht dreimal die Woche zu einer fixen Zeit, um sich selbst einen Rhythmus zu geben.

Diese starke Individualisierung der verschiedenen Fitnesskonzepte ruft nach sehr individuellen Trainingsmöglichkeiten und einer guten Betreuung. Das Personal wird damit zu einem immer wichtigeren Faktor - zum wichtigsten Erfolgsfaktor der Fitnessbranche überhaupt, wie aus dieser Untersuchung herausgeht.

Zusätzlich zu dieser Individualisierung und Komplexitätssteigerung des Fitnessbewusstseins lässt sich auch eine Ausweitung des Fitnessverständnisses in Richtung Gesundheitsbewusstsein feststellen. Über 50\% aller Fitnessbesucher in der Schweiz gaben 1998 als Hauptmotivation für Ihr Fitnesstraining „Gesundheit“ an (BFS, 1998). Nachdem bereits in den 80er Jahren „Gesundheit“ als Hauptmotivation für Fitnesstraining genannt wurde, wurde in den frühen 90er Jahren ,der perfekte Körper“ als einer der Hauptgründe für Fitnesscenterbesuch postuliert. Die vorliegenden Daten zeigen, dass mittlerweile das Pendel zurückgeschlagen hat und Gesundheit ganz klar die Nr. 1-Motivation für Fitnesstraining ist. Figurerhaltung folgt zwar immer noch auf Rang zwei, ist aber weit abgeschlagen. Nur noch $13 \%$ der Kunden geben Figurerhaltung als Hauptmotivation an. 
$\mathrm{Zu}$ einer ganzheitlichen Gesundheit im Sinne der WHO (vollständiges, körperliches, geistiges und soziales Wohlbefinden), gehören aber neben den klassischen Fitnesscenterangeboten Bewegung und Entspannung auch noch andere Bereiche wie Ernährung, soziale Kontakte, psychisches Wohlbefinden, usw. dazu.

Das Fitnesscenter wird damit zu einem von verschiedenen möglichen Partner für die persönliche Gesundheitsförderung. Kooperationen mit anderen Gesundheitsanbietern sind darum in naher Zukunft ein Muss, um die ganzheitlichen Gesundheitsansprüche der Konsumenten zu befriedigen. Ob diese Kooperationen in den gleichen Räumlichkeiten angeboten werden (im Sinne eines Gesundheitszentrums mit integrierter Physiotherapie, Ernährungsberatung, Naturheilarzt, psychologischer Beratung und Arztpraxis) oder geographisch getrennt sind, spielt weniger eine Rolle. Wichtig ist, dass der Kunde eine einzige Anlaufstelle hat, wo er erst beraten und dann an die für ihn optimalen Teildienstleister weitergewiesen wird. Diese Tendenz $\mathrm{zu}$ einem breiteren Gesundheitsbewusstsein wird auch belegt durch die steigende Nachfrage und das immer grössere Angebot an alternativen Gesundheitsangeboten, die über die klassischen medizinischen Möglichkeiten hinausgehen, wie Tai-Chi, Qi Gong, Alexandertechnik, Feldenkrais, Ayurveda, Aura Soma, Akkupunktur, um nur einige zu nennen.

Zusätzlich $\mathrm{zu}$ dieser Verlagerung von Fitnessbewusstsein in Richtung Gesundheitsbewusstsein (Primärprävention) lässt sich ebenfalls beobachten, dass sich immer mehr Kunden nach dem Auftreten von körperlichen Beschwerden zu einem Fitnesscenterbesuch entschliessen (Sekundärprävention). In einzelnen Fitnessstudios beträgt der Anteil an Kunden, die körperliche Beschwerden angeben, bereits über $40 \%$ (SCHMID 1999a). Auch das Bundesamt für Statistik der Schweiz weist in ihrer neuesten Studie über die Gesundheit der Bevölkerung eine Zunahme von Allergien jeglicher Art, körperlichen und oft psycho-somatisch begründeten Leiden sowie Depressionen in den letzten Jahren nach (BFS 1999).

Wer selber einmal von einer Krankheit (hier im weitesten Sinne zu verstehen) betroffen war, der ist sensibilisiert auf die Gesundheit und entwickelt das oben erwähnte Gesundheitsbewusstsein. Es ist bekannterweise eines der Kernprobleme von Public Health Projekten, dass man gesunde Leute nur sehr schwer auf Gesundheit sensibilisieren kann.

Diese körperlich sich äussernden Beschwerden gehen nur zu einem kleinen Teil auf klassische muskuläre Dysbalancen (sitzende und bewegungsarme Gesellschaft) zurück; meist liegen die Probleme tiefer: Arbeitsplatzprobleme, Stress, Mobbing, falsche Einrichtung des Arbeitsplatzes, schlechte Ernährung, Beziehungsprobleme, Elektrosmog, Strahlenbelastung, etc. Diese komplexen Krankheitsbilder können nur mit komplexen Methoden behandelt werden. Das klassische Fitnesstraining ist dabei eine mögliche Komponente. Dessen sollte sich der Fitnesstrainer der Zukunft bewusst sein. Er sollte nicht mehr nur das Fitnesstraining mit seinen Methoden und Trainingsregeln kennen, sondern auch die Grenzen seines Bereiches und die angrenzenden Bereiche. Dies erhöht die Anforderungen an das Personal (und damit auch an die Centerleitung) noch weiter. 
Ausgehend von diesem sich entwickelnden Gesundheitsbewusstsein und den zunehmenden körperlichen Beschwerden der Gesellschaft ergeben sich für das klassische Fitnesscenter mittelfristig zwei mögliche Strategien:

- Konzentration auf das Fitnessgeschäft (Bewegung und Entspannung), klare Abgrenzung gegenüber den angrenzenden Bereichen, aber Partnerschaften mit Anbietern von komplementären Angeboten. An diese Partner können die eigenen Kunden weitergewiesen werden; umgekehrt kommen von diesen Bereichen auch wieder neue Fitnesskunden.

- Integration von Fitnessstudios in ganzheitliche Gesundheitszentren mit einem breiten Angebot an Gesundheitsdienstleistungen und fliessenden Übergängen zwischen den einzelnen Dienstleistern.

Modelle der erstgenannten Strategie kann man bereits an verschiedenen Orten beobachten. Immer mehr arbeiten Fitnessstudios mit Physiotherapien oder Ärzten zusammen. Die Abgrenzungen zwischen medizinischer Trainingstherapie und Fitnesstraining mit einem Schwergewicht in Prävantion und Rehabilitation machen aber in der Praxis sehr Mühe. Die Grenzen werden immer fliessender, was wie schon erwähnt eine grosse Steigerung der Anforderungen an das jeweilige Betreuungspersonal stellt.

Im Bereich der zweiten Strategie stecken die Modellbetriebe noch in den Kinderschuhen. Dem Autor ist ein Fall bekannt, wo in Zürich in nächster Zeit ein Zentrum entstehen soll, wo sich unter dem gleichen Dach eine Arztpraxis mit medizinscher Sporttherapie, eine Physiotherapie, eine psychologische Beratungsstelle, eine Ernährungsberatung, eine Kommunikationsagentur und eine Consultingfirma umfassend um die Gesundheit von Individual- und Firmenkunden kümmern. Mit einem umfassenden Gesundheitsprotokoll werden Defizite und mögliche Ursachen ermittelt und dann die individuellen bereichsübergreifenden Programme entwickelt.

Zusammenfassend kann zu den Wachstumsfaktoren der Fitnessbranche Folgendes gesagt werden:

- Das Fitnessbewusstsein wird abgelöst durch ein Gesundheitsbewusstsein.

- Fitness als Mittel der Sinnfindung und als Stilisierung des perfekten Körpers wird abgelöst durch Fitness (oder eben besser Gesundheit) als tool für bessere Lebensund Alltagsbewältigung.

Es zeichnet sich $a b$, dass die Wachstumsgrenzen im Kernprodukt „Fitnesstraining“ mittelfristig erreicht sind. In Zusammenhang mit der Ausdehnung des Fitnessbewusstseins in Richtung Gesundheitsbewusstsein entstehen aber an den Schnittstellen interessante Wachstumsmärkte.

Weitere Entwicklungsmöglichkeiten ergeben sich im Bereich der betrieblichen Gesundheitsförderung. Immer mehr Firmen entdecken, dass sich mit gesunden Mitarbeitenden viele Ausfälle verhindern lassen. Fitness- und Gesundheitsincentives sind ebenfalls stark im Kommen. Statt monetären Anreizen gibt es Fitnessgutscheine 
oder Trainingsstunden auf Arbeitgeberkosten, wenn die Leistung stimmt. Fitness oder Gesundheit wird auch im Bereich der Corporate Culture eine immer wichtigere Rolle spielen.

Die Schweiz und Europa hinken hier (wie so oft) der Entwicklung in den Vereinigten Staaten einige Jahre hintendrein. Immerhin wurde bereits 1989 von der BUNDESZENTRALE FÜR GESUNDHEITLICHE AUFKLÄRUNG das Thema „Gesundheitsförderung in der Arbeitswelt" aufgegriffen. In den späten 90er Jahren häufen sich dann die Publikationen zu diesem Thema in Europa und auch in der Schweiz (vergleiche GUTZWILLER \& JEANNERET 1996, NIEDER \& SUSEN 1997, RUCKSTUHL et al. 1998).

In der Schweiz konnte man in den letzten drei bis fünf Jahren zusätzlich die Entstehung von verschiedenen Firmen, welche betriebliche Gesundheitsförderung und Public Health anbieten, beobachten (vergleiche KUTLAR 1999). Dies weist ebenfalls daraufhin, dass sich hier ein neuer Markt eröffnet, der auch für Fitnesscenter Chancen bietet.

\subsection{Erkenntnisse über die Übertragbarkeit der Ergebnisse}

In der Einleitung wurden folgende Fragen aufgeworfen:

- Sind die Zusammenhänge von Persönlichkeit, Führungsverhalten und Erfolg auf andere Branchen übertragbar und decken sich die Anforderungen an Fitnesscentermanager mit den Anforderungen anderer Dienstleistungsbranchen?

- Wirken sich gesellschaftliche Veränderungen auf das Anforderungsprofil von Fitnesscentermanagern aus?

Aufgrund der vorliegenden Untersuchungsergebnisse können folgende Aussagen gemacht werden:

\subsubsection{Management-Anforderungen in der Dienstleistungsbranche}

In der Untersuchung ging der ESTJ-Typ als Erfolgs-Fitnessmanager hervor. Dieser klassische Manager-Typ, der sich vor allem durch Effizienz und eine Optimierung von Input und Output auszeichnet, wird auch an anderen Orten (RÜHLI 1985) als Erfolgstyp bezeichnet, jedoch eher im Bereich der Industrie. In der Dienstleistungsbranche wird eher der NT- oder NF-Typ als Erfolgstyp favorisiert, der schnell und intuitiv wahrnimmt, auch um Effektivität besorgt ist und entweder sachlich oder emotional entscheidet.

Dass momentan noch der ESTJ-Manager in der Fitnessbranche erfolgreicher ist als der ENTJ oder ENFJ-Typ kann damit erklärt werden, dass das Bedürfnis „Fitnesstraining“ noch wenig differenziert und individuell daherkommt und damit wenig Spielraum für den effektiven Manager besteht. Die Fitnessstudios gleichen sich im Angebot relativ stark, womit es wichtiger ist, die vorhandene Infrastruktur optimal auszunützen, als eine optimale Infrastruktur zu schaffen.

Die beschriebene Veränderung des Fitnessbewusstseins in Richtung Gesundheitsbewusstsein wird diese Situation aber stark ändern. Plötzlich werden originelle und 
individuelle Lösungen, Kooperationen mit anderen Anbietern und eine starke Kundenorientierung gefragt sein - und da ist dann der ENTJ oder ENFJ-Typ erfolgreicher.

Als Vergleichsbranche aus dem Dienstleistungsektor bietet sich das Gastgewerbe, im Besonderen die Hotellerie, an. Die Hotelbranche lässt sich vom Produkt her sehr gut mit der Fitnessbranche vergleichen: Anbieten einer Infrastruktur für zielgerichtete Aktivitäten (Fitness: Training, Hotel: Seminare) oder für Freizeit-Zwecke (Fitness: Entspannung, Kontakte, Erholung, Hotel: Ferien, Erholung), wobei das Personal eine äusserst wichtige Komponente im Angebot ist.

In der Hotelbranche (REHKUGLER, 1997) werden denn auch die gleichen drei wichtigsten Erfolgsfaktoren genannt, wie sie in dieser Untersuchung für die Fitnessbranche ermittelt wurden, und erst noch in der gleichen Reihenfolge:

- Freundlichkeit des Personals

- Qualität und Kompetenz des Personals

- Sauberkeit

Ähnlich wie dies im Fitnessbereich mit den Geräten aufgezeigt werden konnte, kommt auch auch in der Hotelbranche die eigentliche Infrastruktur des Hotels (Zimmer, Lounge, Zusatzangebote) erst an fünfter Stelle.

Was die Konkurrenzangst anbelangt, zeigen sich ebenfalls Ähnlichkeiten. Auch im Hotelbereich ist gemäss REHKUGLER (1997) die Bereitschaft, Zahlen herauszugeben, sehr gering.

In der Entwicklung der Anforderungen ans Management ergeben sich ebenfalls Parallelen. Noch vor wenigen Jahren ging es in der Hotelbranche vor allem darum, die vorhandene Infrastruktur optimal auszulasten. Heute legen aktuelle Stelleninserate für Hotelmanager grossen Wert auf Innovationsfreude, Trendbewusstsein, Marketingverständnis, Personalführungserfahrung und DienstleistungsbereitschaftAttribute, die in Kürze auch von Fitnesscentermanagern gefordert werden.

Gerade im Bereich der Dienstleistungsbereitschaft kann die Schweiz noch sehr viel lernen. Die Zeit des Gastes als Melkkuh ist definitiv vorbei. Die grosse Baisse im Schweizer Tourismus in den letzen Jahren ist neben den hohen Preisen und damit einem schlechten Preis-/Leistungsverhältnis zu einem grossen Teil mit dieser mangelnden Dienstleistungsbereitschaft und der fehlenden echten Gastfreundschaft zu erklären. Mittlerweile hat man die Zeichen der Zeit erkannt und gibt sich viel Mühe - mit Erfolg wie die Zahlen zeigen (SCHWEIZ TOURISMUS 1999).

Eine ähnliche Entwicklung wird sich in der Fitnessbranche abspielen. Nur das Anbieten einer guten Infrastruktur wird sehr schnell nicht mehr genügen. Echte Dienstleistungsbereitschaft und ungeheucheltes Kundeninteresse sind gefragt. So wie dies von grossen Firmen anderer Dienstleistungsbranchen, wie McDonalds oder McKinsey, in ihren Leitbildern von Ihren Mitarbeitenden bereits heute gefordert wird. 


\subsubsection{Gesellschaftliche Veränderungen und ihre Auswirkungen}

Im vorherigen Unterkapitel wurden die gesellschaftlichen Veränderungen im Hinblick auf ein komplexes und individuelles Gesundheitsbewusstsein als tool für die persönliche Lebensbewältigung aufgezeigt.

Diese Veränderungen beeinflussen alle Bausteine unseres Führungsmodells.

Im Bereich der Führungs-Persönlichkeit wurden bereits Aussagen gemacht und eine Verschiebung in Richtung innovativ-intuitiver Typ postuliert.

Im Bereich des Führungsverhaltens wird Personal führen (bereits jetzt schon die wichtigste Führungstätigkeit) noch an Bedeutung gewinnen, da Qualität und Freundlichkeit des Personals weiterhin die wichtigsten Erfolgsfaktoren bleiben werden. Probleme lösen im Sinne von die Probleme der Kunden lösen wird ebenfalls noch wichtiger werden. Um Probleme zu lösen, muss man sie ja bekanntlich zuerst wahrnehmen, analysieren und dann entscheiden. Je komplexer die Probleme sind, desto wichtiger wird die intuitive Aufnahmefähigkeit (N-Typ), die über die sensitive Wahrnehmung (S-Typ) hinausgeht und hier nochmals die Tendenz Richtung NPersönlichkeit unterstreicht.

Die Bedeutung von Marketing wird ebenfalls noch zunehmen. Je individueller die Angebote werden, desto wichtiger wird die Positionierung und die klare, aber auch kreative und innovative Kommunikation der USPs.

Im Bereich des Erfolgs wird es zentral wichtig sein, die sich abzeichnenden Veränderungen in der Gesellschaft schnell zu erkennen und möglichst adäquate Lösungen für die Kunden zu finden. Die Qualität des Personal wird weiterhin eine tragende Säule des Erfolgs sein. Je mehr sich die Grenzen zwischen den einzelnen Gesundheitsanbietern aufweichen, desto wichtiger wird die Schulung der Mitarbeitenden an diesen Schnittstellen. Die Planung und Realisation dieser Schulung stellt wiederung sehr hohe Anforderungen an den Manager, da er in der Lage sein muss, von allen möglichen Schnittstellen die erfolgsreleventesten herauszufinden und dort Prioritäten zu setzen. Dies setzt einen sehr breiten eigenen Horizont voraus.

Neu könnte ein Faktor unabhängige Beratung dazukommen. Je unübesichtlicher der Markt wird (vgl. Liberalisierung des Versicherungsmarktes), desto wichtiger wird für den Kunden eine unabhängige Erstberatungsstelle, die ihm die für ihn optimalen Gesundheitsdienstleistungen vermitteln.

Wahrscheinlich wird auch die Bedeutung der Kennzahlen eher zurückgehen. Da die Betriebe immer individueller werden, können sie auch immer schlechter miteinander verglichen werden. Da Branchen-Kennzahlen ja die Generalisierung schlechthin sind, werden sie für den einzelnen Betrieb immer weniger eine mögliche Entscheidungsgrundlage sein können.

Im Bereich der Situation kann man Folgendes festhalten:

Der Markt für das Kernprodukt „Fitnesstraining“ ist beinahe gesättigt, wie bereits in Kapitel 4.1 Die Fitnesslandschaft Schweiz (Seite 23) beschrieben wurde. Eigenes Wachstum kann dort fast nur noch auf Kosten der Konkurrenz und damit nur mit grossem Aufwand realisiert werden, womit sich die Anstrengungen der verschiedenen 
Fitnessanbieter vor allem in Richtung der Schnittstellenbereiche mit Gesundheit bewegen werden. Damit einher geht eine Entspannung des Konkurrenzdruckes im Kernprodukt, aber eine Verlagerung in Richtung Prävention und Rehabilitation. Dies ruft wiederum nach massiven Investitionen, da die konventionellen Fitnessgeräte für therapeutische Zwecke zu wenig geignet sind. Wenn man nicht selbst diese Investitionen aufbringen kann, sind Kooperationen mit Partnern in den Schnittstellenbereichen eine gute Möglichkeit.

Es setzt damit nach der ersten Strukturbereinigung ,qualitativ gute Center gegenüber schlechten Centern" in den frühen 90er Jahren eine zweite Welle der Strukturbereinigung ein: „Integrierte Gesundheitszentren gegenüber klassischen Fitnesscentern“. Wobei bei dieser zweiten Welle im Gegensatz zur ersten Strukturbereiningung beide Strukturen mit ihren unterschiedlichen Strategien Marktchancen haben.

Wie bereits an anderer Stelle erwähnt, wird die Kompetenz und Freundlichkeit des Personals als kritischer Erfolgsfaktor noch bedeutungsvoller werden.

Die Faktoren Abo-Möglichkeiten und Angebot (insbesondere die Kombinationen mit den anderen Gesundheitsanbietern) werden tendenziell wichtiger, die Faktoren Maschinen und Räumlichkeiten eher weniger wichtig.

Grundsätzlich lassen sich also die in der vorliegenden Untersuchung herausgefundenen Zusammenhänge und Tendenzen in einer allgemeinen Art auch auf andere Branchen im Dienstleistungsektor übertragen, beziehungsweise entsprechen den dort herrschenden Verhältnissen. 


\section{Zusammenfassung und Ausblick}

\subsection{Erkenntnisse über die Zusammenhänge zwischen den einzelnen Bausteinen}

Bei der Einleitung wurden folgende praktische Fragen ins Zentrum des Interessens dieser Untersuchung gerückt:

- Gibt es Zusammenhänge zwischen Persönlichkeit, Sportverständnis, Führungsverhalten und dem Erfolg eines Fitnesscenter-Managers?

- Wenn ja, welche Kriterien oder Faktoren (einzeln oder in Kombination) erhöhen die Chance des Erfolgs?

- Lässt sich ein charakteristisches Persönlichkeits- oder Führungsprofil des erfolgreichen und des nicht-erfolgreichen Fitnesscenter-Managers zeichnen?

Einzelne konkrete Antworten konnten denn auch gegeben werden (vergleiche jeweilige Kapitel). In der Übersicht kann das Ausgangsmodell (vergleiche Abbildung 3-2 Arbeitsmodell der vorliegenden Arbeit wie folgt bestätigt werden:

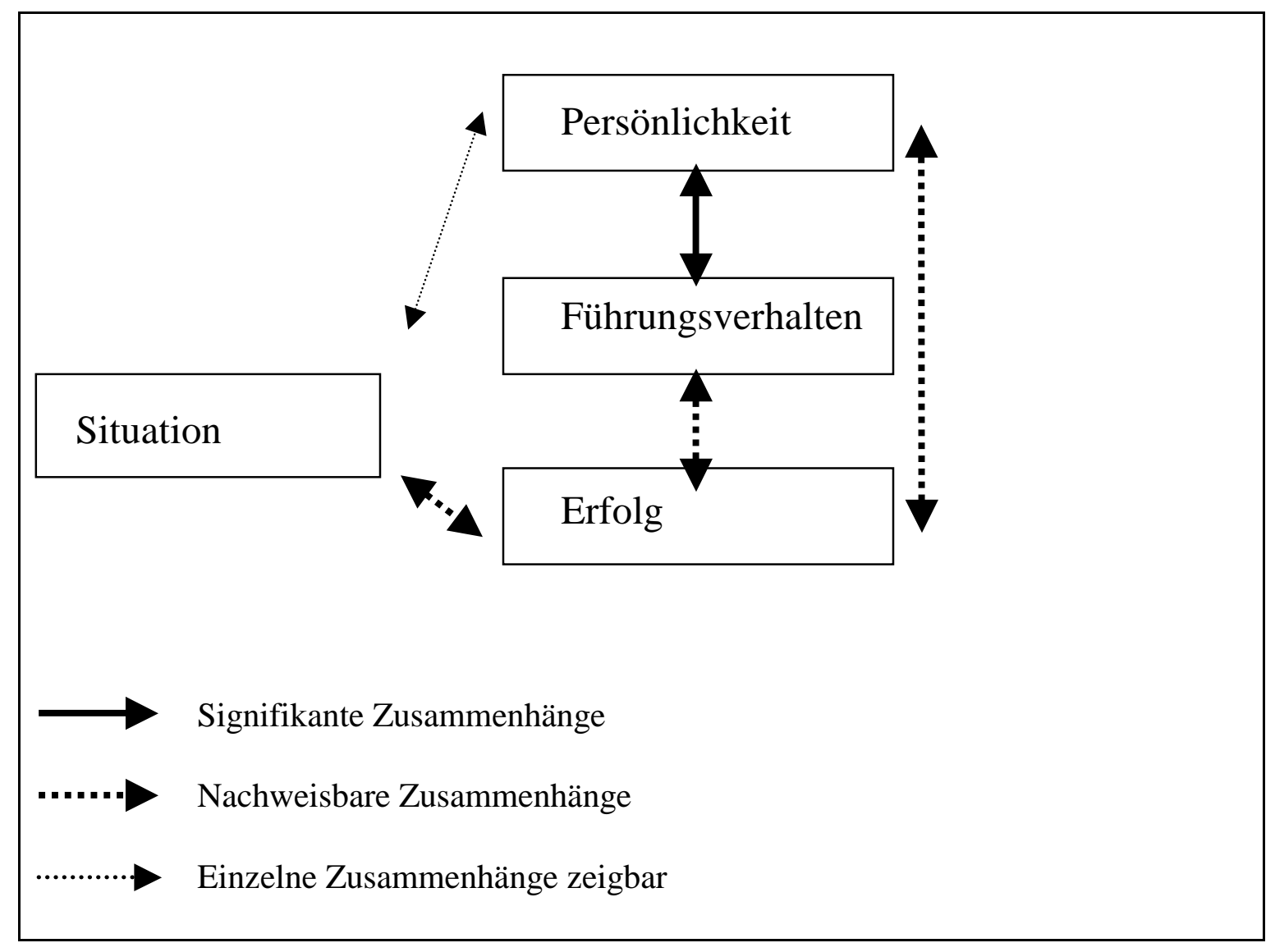

Abbildung 21-1 Zusammenfassung 


\section{Persönlichkeit und Führungsverhalten}

Zwischen Persönlichkeit und Führungsverhalten konnten signifikante Zusammenhänge gezeigt werden. Es hat sich auch herausgestellt, dass der MBTI-Typenindikator ein sehr geeignetes Mittel, um Verhaltensprognosen zu machen.

Folgende Zusammenhänge konnten signifikant nachgewiesen werden:

- I-Typen verwenden signifikant mehr Zeit für Planen/Koordinieren und Administration/Papierarbeit als E-Typen.

- N-Typen verwenden signifikant mehr Zeit für Motivieren/Bestärken und Akquisition/Marketing als S-Typen.

- J-Typen verwenden signifikant mehr Zeit für Planen/Koordinieren und Administration/Papierarbeit als P-Typen.

Es muss aber in diesem Zusammenhang nochmals mit aller Deutlichkeit darauf hingewiesen werden, dass von der Persönlichkeit her nie direkt und im Sinne einer Ursache-Wirkung-Beziehung auf Verhalten geschlossen werden kann. Die Persönlichkeit stellt eine Grunddisposition dar, die das eine oder andere Verhalten eher begünstigt oder eben eher unwahrscheinlich macht. Situative Umstände (Tagesform, Stress, Marktsituation, rechtliche Lage, Umwelt, etc.) beeinflussen das Verhalten innerhalb dieser Grunddisposition noch sehr stark. Gewisse Verhalten, die eher nicht dem Typ entsprechen, sind auch absolut lernbar und nicht mit dem Persönlichkeitstypen erklärbar.

\section{Führungsverhalten und Erfolg}

Auch hier konnten Zusammenhänge aufgezeigt werden. Grundsätzlich waren aber wenig signifikante Korrelationen nachweisbar. In diesem Bereich zeigen sich die Grenzen der quantitativen Forschung. Gerade im Führungsverhalten kommt es nicht nur auf die Menge der Führungstätigkeiten an, sondern sehr stark auf deren Art und Weise. Immerhin konnten Führungstätigkeiten aufgezeigt werden, die in der Fitnessbranche erfolgversprechender sind als andere:

- Ausbilden

- Planung, Administration

- Probleme lösen

Es konnte auch gezeigt werden, dass sehr erfolgreiche Manager vor allem in den folgenden Führungstätigkeiten mehr Zeit investieren als ihre wenig erfolgreichen Konkurrenten:

- Ausbilden

- Konfliktmanagement

- Personalführung

\section{Persönlichkeit und Erfolg}

Es ist nicht möglich (zumindest nicht mit dieser Untersuchungsanlage), zu entscheiden wie stark dass die Persönlichkeit direkt auf den Erfolg wirkt und wie stark dass sie sich indirekt über das Führungsverhalten auf den Erfolg auswirkt. Es konnte gezeigt werden, 
dass gewisse Persönlichkeitstypen von ihrer Veranlagung oder eben von ihrer Persönlichkeit her eher dazu neigen, die in der Fitnessbranche als erfolgreich herausgeschälten Führungstätigkeiten auszuführen und daher mit weniger Aufwand zum Erfolg kommen als andere.

Insbesondere konnte ein Prototyp des erfolgreichen Fitnesscenter-Managers bestimmt werden: der ESTJ-Typ. Es wurde aber auch aufgezeigt, dass sich der Erfolgstyp mit sich veränderndem Umfeld ebenfalls verändern muss und wird.

\section{Situation und Erfolg}

Wie bereits oben erwähnt, hat die jeweilige Situation einen grossen Einfluss auf das Verhalten und damit indirekt auf den Erfolg. Die Situation hat aber auch direkt einen Einfluss auf den Erfolg. Auch stellt sich wieder die ähnliche Frage wie beim Zusammenhang zwischen Persönlichkeit und Erfolg, wie stark die Situation direkt auf den Erfolg wirkt und wie stark sie indirekt über das Verhalten wirkt. Auch hier muss diese Frage unbeantwortet bleiben.

Folgende kritische Erfolgsfaktoren konnten herausgefunden werden:

- Kompetenz des Personals

- Freundlichkeit des Personals

- Sauberkeit der Nasszone

- Breite des Angebots

- Moderne Maschinen und Geräte

- Anzahl Parkplätze

Es konnte auch gezeigt werden, dass die obengenannten Erfolgsfaktoren durch die sehr erfolgreichen Center markant besser erfüllt werden als durch die wenig erfolgreichen.

Zusammenfassend lässt sich feststellen, dass es gelungen ist, einzelne Zusammenhänge auch statistisch nachzuweisen. Generell muss aber bemerkt werden, dass die vorliegende Studie ein quantitatives Vorgehen gewählt hat und deshalb auch nur Antworten auf quantitative Fragen zu erwarten sind.

Die untersuchten Begriffe wie Persönlichkeit, Führungsverhalten und Erfolg haben jedoch allesamt auch eine qualitative Seite, die nicht erfasst werden konnte. Zum Beispiel im Bereich Führungsverhalten konnten quantitativ wenige erfolgsrelevante Führungstätigkeiten eruiert werden. Erfolgreiche und nicht erfolgreiche Führungspersönlichkeiten tun oftmals dasselbe und erzielen dabei sehr unterschiedliche Effekte.

Der qualitative Bereich des Führungsverhaltens, aber auch der Persönlichkeit oder des Erfolgs sind denn auch die Stossrichtungen zukünftiger Forschungsarbeiten, die auf der vorliegenden Studie aufbauen wollen.

Die einzelnen unbeantworteten oder überhaupt erst aufgetauchten Fragen sind in den jeweiligen Kapiteln erwähnt und sollen an dieser Stelle nicht nochmals im Detail zitiert werden. 


\subsection{Vergleich der Gruppen Stadt - Land und Gross - Klein}

Mit der Bildung von zwei besonderen Untergruppen Center auf dem Land und Center in der Stadt sowie grosse und kleine Center wurde versucht, zusätzliche Erkenntnisse zu gewinnen und Ansatzpunkte für weitere Studien zu eruieren.

$\mathrm{Zu}$ den folgenden Punkten konnten einige interessante Zusammenhänge aufgezeigt werden, die in weiteren Untersuchungen noch detaillierter angegangen werden können.

\section{Konkurrenzdruck}

Der Konkurrenzdruck wird von den Stadtcentern tendenziell signifikant kleiner eingeschätzt als durch die Landcenter, obwohl die Fitnesscenter-Dichte in der Stadt effektiv viel grösser ist als auf dem Land. Eine erfolgreiche Nischenpolitik ist offenbar dafür verantwortlich.

Bei den Gruppen grosse und kleine Center sind keine Unterschiede im Bereich des Konkurrenzdruckes nachzuweisen.

\section{Fläche und Personal}

Die Center in der Stadt sind flächenmässig tendenziell signifikant grösser als die Center auf dem Land. In den Centern der Stadt ist ebenfalls signifikant mehr Personal vorhanden als auf dem Land. Im Bereich der Vollzeitangestellten hat es sogar höchst signifikant mehr Personal. Offensichtlich braucht es in der Stadt mehr Betreuung, um den Anforderungen der Kunden und dem grösseren effektiven Konkurrenzdruck standzuhalten.

Ähnliches lässt sich über die Unterschiede von kleinen zu grossen Centern sagen. Die grossen Center mit mehr Fläche haben signifikant mehr Personal als die kleineren Center.

\section{Kunden und Abonnementspreise}

Center in der Stadt oder grössere Center haben im Gegenzug aber nicht wie erwartet würde, auch mehr Kunden. Dies bedeutet, dass die höheren Fixkosten auf gleich viele Kunden verteilt werden müssen. Dies wiederum hat wahrscheinlich einen höheren Abonnementspreis zur Folge. Die Abonnementspreise wurden in dieser Studie nicht erhoben, womit auf diese Frage keine Antwort gegeben werden kann. Dies wäre aber sicher eine interessante Fragestellung für weiterführende Untersuchungen.

\section{Manager-Persönlichkeit}

Sowohl bei den Stadt- und Land-, als auch bei den grossen und kleinen Centern lassen sich zwar einzelne Unterschiede, aber keine signifikanten Zusammenhänge im Bereich der Manager-Persönlichkeit feststellen.

\section{Führungsverhalten}

Sowohl die Stadt-Manager als auch die Manager grosser Center schätzen die Wichtigkeit von Administration/Papierarbeit tendenziell signifikant wichtiger ein als die Vertreter der zweiten Gruppe. 
In der effektiven Führungstätigkeit sind keine Unterschiede zwischen Stadt- und Landcentern zu beobachten. Grosse Center wenden höchst signifikant mehr Zeit auf für Personalführung, was bei mehr Angestellten auch durchaus verständlich ist.

\section{Erfolgseinschätzung}

Die Manager der grösseren Center schätzen ihren Erfolg tendenziell signifikant höher ein als die Manager von kleinen Centern, wo hingegen bei den Stadt- und Landcentern keine Unterschiede nachgewiesen werden konnten. 


\subsection{Erkenntnisse über die Wachstumsfaktoren der Branche}

Die Fitnessbranche hat weiterhin Wachstumspotential. Dieses Wachstum wird aber weniger im herkömmlichen Kerntätigkeitsbereich der Fitnesscenter stattfinden, nämlich dem Angebot von Bewegung und Entspannung, sondern vor allem in den Schnittstellenbereichen von Fitness zu Prävention, Rehabilitation und ganzheitlicher Gesundheit.

Die in der Einleitung (vergleiche Kapitel 1.2 Wachstumsfaktoren der Fitnessbranche, Seite 3) erwähnten Wachstumsfaktoren

- Entwicklung des Fitnessbewusstseins in der Bevölkerung

- Persönliche Fitness als Mittel der Sinn- und Identitätsfindung

- Stilisierung des perfekten Körpers

werden deshalb in den Hintergrund gedrängt oder sogar abgelöst durch neue Wachstumsfaktoren.

Die Entwicklung des Fitnessbewusstseins in der Bevölkerung ist in einer ersten Phase abeschlossen und wird durch die Entwicklung eines ganzheitlichen Gesundheitsbewusstseins, das über Fitness hinausgeht, abgelöst.

Dieses Gesundheitsbewusstsein wird gefördert durch zunehmend ungesundere Lebensverhältnisse in der modernen Welt (sowohl privat als auch geschäftlich) und damit verbundene persönliche Erfahrungen mit zilisatorischen Krankheiten wie HerzKreislauf-Problemen, psycho-somatischen Leiden, Allergien, Depressionen, usw.

Dieses komplexere Fitness- und Gesundheitsverständnis ruft nach komplexeren Angeboten als nur Fitness und eröffnet an den Schnittstellen zur Medizin (Prävention und Rehabilitation) und in den Grenzbereichen zur alternativen Medizin interessante Wachstumsmöglichkeiten für die Branche. Dies ist auch nötig, da sich im klassischen Kernprodukt „Fitnesstraining“ die Wachstumsmöglichkeiten beinahe erschöpft haben.

Diese neuen Märkte stellen aber auch ganz neue Anforderungen an das Personal und die Fitnesscenter-Manager. Das Fitnesscenter wird $\mathrm{zu}$ einem unter vielen möglichen Anbietern auf dem Gesundheitsmarkt und muss sich klar positionieren oder in Kooperationen mit Anbietern der anderen Bereiche zu einem Generalunternehmer in Sachen Gesundheit werden.

Die zunehmende Sensibilisierung von Firmen für die Gesundheitssituation der Mitarbeitenden eröffnet den interessanten Markt der betrieblichen Gesundheitsförderung, der stark am Wachsen ist.

Fitness als Mittel der Sinnfindung und als Stilisierung des perfekten Körpers wird abgelöst durch Fitness oder eben besser Gesundheit als tool für bessere Lebens- und Alltagsbewältigung.

Gerade bei von Zivilisationskrankheiten betroffenen Personen setzt sich die Erkenntnis durch, dass mit gesundheitsfördernden Massnahmen (die aber viel weiter gehen als „nur“ Fitnesstraining) die Gesundheit stark beeinflusst werden kann und sich mit besserer Gesundheit die Anforderungen des täglichen Lebens viel besser bewältigen lassen. 


\subsection{Erkenntnisse über die Übertragbarkeit der Ergebnisse}

Viele Ergebnisse decken sich mit Erkenntnissen anderer Branchen. Gerade die Hotelbranche ist dabei eine gute Vergleichsbranche. Das angebotene Produkt ist dem der Fitnessbranche auch sehr ähnlich. Im Bereich des Erfolgs decken sich die wichtigsten Erfolgsfaktoren, die in dieser Untersuchung ermittelt werden konnten, exakt mit den Erfolgsfaktoren der Hotelbranche. Im Bereich der Persönlichkeit und des Führungsverhaltens lassen sich ebenfalls Parallelen aufzeigen. Derselbe Wandel vom effizienten Ressourcenmanager zum effektiven, kundenorientierten Dienstleistungsprofi, der sich in den letzten Jahren in der Hotelbranche und in anderen Dienstleistungsbranchen vollzogen hat, ist auch in der Fitnessbranche zu beobachten. In diesem Sinne sind die vorliegenden Erkenntnisse keine Überraschungen, sondern decken sich mit dem allgemeinen Trend.

Die beschriebenen Veränderungen in der Gesellschaft und die damit verbundene Verschiebung des Fitnessbewusstseins hin zu einem integralen Gesundheitsbewusstsein haben Auswirkungen auf alle Bereiche unseres Untersuchungsmodells.

Im Bereich der Persönlichkeit wird eine Entwicklung in Richtung intuitiv-innovativer und kundenorientierter Dienstleister einsetzen, beziehungsweise wird sich diese Entwicklung noch verstärken.

Im Bereich des Führungsverhaltens wird das Personalmanagement, das Marketing und zukunftsorientierte Problemlösungsstrategien noch wichtiger werden.

Im Bereich des Erfolgs wird entscheidend sein, inwiefern und wie glaubwürdig man die sich abzeichnenden Schnittstellenbereiche zu anderen Gesundheitsanbieter in das eigene Angebot integrieren kann, um die sich abzeichnende Sättigung des Kerngeschäfts „Fitnesstraining“ aufzufangen.

Im Bereich der Situation wird das Personal im Sinne von umfassender Beratung und Betreuung der wichtigste Erfolgsfaktor bleiben. Zusätzlich werden Abonnementsmöglichkeiten und Angebot (inbesondere die Kombinationsmöglichkeiten mit anderen Gesundheitsanbietern) noch wichtiger werden.

Diese Feststellungen unterstützen die in dieser Arbeit herausgefundenen Zusammenhänge zwischen den einzelnen Bausteinen unseres Modells. Wenn sich einzelne Bausteine ändern, hat das einen Einfluss auf alle anderen Bausteine und wenn sich die Umwelt ändert, so beeinflusst dies das ganze System

Die Zusammenhänge und gegenseitigen Wechselwirkungen der einzelnen Bausteine bleiben tendenziell konstant und sind auch auf andere Branchen übertragbar.

Die effektiven, in dieser Arbeit ermittelten Zahlen und Facts zu den einzelnen Bausteinen stellen eine Momentaufnahme der jetztigen Situation der Fitnessbranche dar und müssen auf dem Hintergrund einer sich permanent ändernden Umwelt und Gesellschaft immer wieder neu ermittelt werden.

Und diese Umwelt und Gesellschaft wird sich in nächster Zeit immer schneller verändern... 


\section{Literaturverzeichnis}

ANTHONY, R. N., DEARDEN, J. \& BEDFORD, N. M. (1989). Management Control Systems. 6. Auflage. Homewood: Illinois.

ATAG, SHZ, IHA (1988). Schweizerische Gehaltsumfrage Führungskräfte. Hergiswil.

BADELT, C. (1999). Handbuch der Nonprofit Organisation. Stuttgart: SchäfferPöschel.

BANDURA, A. (1986). Social foundations of thought and action: A social cognitive theory. Englewood Cliffs, NJ: Prentice Hall.

BASS, B. M. \& AVOLIO, B. J. (1993). Transformational leadership: A response to critiques. In M. M. CHEMERS \& R. AYMAN (Eds.), Leadership theory and research: Perspectives and directions (pp. 49 -80). San Diego, CA: Academic Press.

BENNINGHAUS, H. (1986). Soziale Einstellung und soziales Verhalten. In ALBRECHT, G., DAHEIM, H. J. \& SACK, F. (Hrsg.) Soziologie. Opladen.

BENTS, R. \& BLANK, R. (1995). Myers-Briggs-Typenindikator. Göttingen: BeltzTest.

BELTZ MBTI TESTMANUAL (1995). Göttingen: Beltz-Test.

BFS Bundesamt für Statistik (1998). Schweizerische Gesundheitsbefragung. In Pressemitteilung 108/1998. Bern: BFS.

BFS Bundesamt für Statistik (1999). Bevölkerung und Gesundheit. Bern: BFS.

BIRD, A. M. (1977). Development of a model for predicting team performance. Research Quarterly, 48, pp. 24 -32.

BLAIR, S. (1989). Good Sense - Good Health. In Sports Illustrated, 13.11.89.

BLAKE, R. R. \& MOUTON, J. (1986). Verhaltenspsychologie im Betrieb. Düsseldorf: Econ.

BODYLIFE (1995). Der Fitnessboom. Fachzeitschrift der Fitnessbranche 03/04 1995, S. 149 - 151.

BORTZ, J. (1984). Lehrbuch der empirischen Forschung der Sozialwissenschaften. Berlin. 
BUSSON R. S., LARSON, L. L., VICARS, W. M., \& NESS, J. J. (1981). The nature of the police executives work: Final report. Carbondale, IL: Southern Illinois University.

BOUCHARD, C. et al. (1990). Exercise, Fitness and Health. Illinois: Human Kinetics.

BRANSTÄTTER H. (1993). Persönliche Leistungs- und Verhaltensbedingungen. In H. Schuler (Hrsg.), Lehrbuch Organisationspsychologie (S. 213 - 233). Bern: Huber.

BRIGGS, K. C. \& MYERS, I. B. (1977). Myers-Briggs Type Indicator. Palo Alto, CA: Consulting Psychologists Press.

BROMME, R. (1992). Der Lehrer als Experte. Bern: Haupt.

BRUHN, M. (1991). Dienstleistungsqualität: Konzepte - Methoden - Erfahrungen. Wiesbaden: Gabler.

BUNDESZENTRALE FÜR GESUNDHEITLICHE AUFKLÄRUNG in Zusammenarbeit mit der WHO/Europa (Hrsg.). (1989). Gesundheitsförderung in der Arbeitswelt. Berlin.

CACHAY, K. \& CAHAI, E. (1989). Brauchen Trainer Pädagogik? Leistungssport 19, 5, S. $26-30$.

CACHAY, K. (1995). Gesellschaft - Sport - Trainer. Systemtheoretische Überlegungen zu Perspektiven der Trainerrolle. Leistungssport 6, S. 23 - 26.

CARLYLE, T. (1841). Heroes and hero worship. Boston: Adams.

CARLSON, S. (1951). Executive Behaviour: A Studdy of the Work Load and Working Methods of Managing directors. Stockholm.

CHELLADURAI, P. (1980). Leadership in sports organizations. In: Canadian Journal of Applied Sport Sciences, 5, pp. 226 - 231.

CHELlADURAI, P. (1985). Sportmanagement: Macro Perspectives. London: Sports Dynamics.

CHELladURAI, P. \& CARRON, A. (1981). Applicability to youth sports of the Leadership Scale for Sports. In: Perceptual and Motor Skills, 53, pp. 361-362.

CHElladURAI, P. \& KUGA, D. J. (1996). Teaching and Coaching: Group and Task Differences. In: QUEST, 48, S. 470 - 485.

DIETERICH, R. \& SOWARKA, B. H. (1994). Gesamtkonzepte der Persönlichkeit. In W. SARGES (Hrsg.), Management-Diagnostik, 2. vollst. überarbeitete Auflage (S. 432 - 444). Göttingen: Hogrefe. 
DIETRICH, K., HEINEMANN, K. \& SCHUBERT, M. (1990). Kommerzielle Sportanbieter. Schorndorf: Hofmann.

DSSV Deutscher Sportstudio verband (1998). Eckdaten 1996. www.dssv.de (Stand 21.9.99).

EIGENMANN, P. (1997). Die Centerbranche im Gesundheitsmarkt. Winterthur: Qualitop.

EIGENMANN, P. (1998). Entwicklungen in der Centerbranche. Winterthur: Qualitop.

FAYOL, H. (1916). Administration industrielle et génerale. Paris.

FIEDLER, F. E. (1967). A theory of leadership effectiveness. New York: McGrawHill.

FIEDLER, F. E. (1987). Führungstheorien - Kontingenztheorien. In A. KIESER, G. REBER \& R. WUNDERER (Hrsg.), Handwörterbuch der Führung, S. 809 - 823. Stuttgart, Poeschel.

FIXX J. F. (1977). The Complete Bok of Running. New York, Random House.

FLEISHMAN, E. A., ZACCARO, S. J. \& MUMFORD, M. D. (1991). Individual differences in leadership: An overview. Leadership Quaterly, 2, 237-243.

FRANKE, E. (1996). Zum Selbstbild des Trainerberufs im Spiegel seiner Verantwortung. Leistungssport 19, 1, S. $21-24$.

FRIEDBERG, A. (1984). Facts on File Dictionary of Fitness. New York, Facts of File.

FRIEDMANN, M. \& ROSENMANN, R. H. (1974). Type A Behaviour and your heart. New York: Knopf.

GABLER, H. \& HAHN, E. (1995). Zur Person des Trainers - Überlegungen auf der Grundlage einer Untersuchung an Bundestrainern. In Leistungssport 19, 5, S. 26 -30.

GALTON, F. (1869). Hereditary genius. New York: Appleton.

GARDNER, L. W. \& MARTINKO, M. J. (1990). The relationship between psychological type, managerial behavior and managerial effectiveness: an empirical investigation. In Journal of Psychological Type 19, pp.34 - 41.

GISOLFI, C. V. \& LAMB, D. R. (1989). Exercise and Sport. Indianapolis.

GLASSNER, B. (1989). Fitness and Postmodern Self. In Journal of Health and Social Behaviour Vol. 30, pp. 180 -191. 
GOLDBERG, L. R. (1990). An alternative „description of personality“: The big five factor structure. Journal of Personality and Social Psychology, 59, pp. 1216 - 1229.

GOUGH, H. G. (1952). The Adjective Check List. Palo Alto, CA: Consulting Psychologists Press.

GOUGH, H. G. \& HEILBRUNN, A. B. (1980). The Adjective Check List Bibliography 1980 Edition. Palo Alto, CA: Consulting Psychologists Press.

GOUGH, H. G. (1984). A managerial potential scale for the California Psychological Inventory. Journal of Applied Psychology, 69, pp. 233 - 240.

GRANTHAM, W.C. (1998). Health Fitness Management: a comprehensive resource for managing and operating programs and facilities. Champaign: Human Kinetics.

GULICK, L. \& URWICK, L. (Eds.) (1937). Papers on the Science of Administration. New York.

GUTZWILLER, F. \& JEANNERET, O. (Hrsg.) (1996). Sozial- und Präventivmedizin Public Health. Bern: Hans Huber.

HAGEDORN, G. (1995). Trainer - die vereinigten Widersprüche der Persönlichkeit. Leistungssport 19, 3, S. $33-36$.

HENDRY, L. B. (1972). The Coaching Stereotype. In W. T. Whiting (Ed.) Readings in Sport Psychology. London: Kimpton.

HENDRY, L. B. (1974). Coaches and Teachers of Physical Education: A comparison of the personality dimensions underlying their social orientation. International Journal of Sport Psychology, 5 (1), pp. 40 - 53.

HELLRIEGEL, D \& SLOCUM, J. W. (1992). Management $\left(6^{\text {th }}\right.$ ed.). Reading, Mass: : Addison-Wesley.

HERRON, N. L. (Ed.) (1992). The Leisure Literature. A Guide to Sources in Leisure Studies, Fitness, Sports and Travel. Englewood, Colorado: Libraries unlimited.

HERSEY, P. \& BLANCHARD, K. H. (1977). Management of organisational behavior: utilizing human ressources. Englewood Cilffs, N. J.: Prentice-Hall.

HODGE, B. J. \& ANTHONY, W. P. (1988). Organization Theory, 3. Auflage. Boston, Massachusetts: Prentice Hall.

HOFSTÄTTER, P. R. (1966). Einführung in die Sozialpsychologie. Stuttgart: Kröner. 
HOGAN, R. T. (1991). Personality and Personality Measurement. In M. D. DUNETTE \& L. M. HOUGH (Eds.), Handbook of industrial and organizational psychology, Vol. 2, $2^{\text {nd }}$ Ed. (pp. 873 - 909). Palo Alto, CA: Consulting Psychologists Press.

HORN, T. S. (1992). Leadership Effectiveness in the Sport Domain. In T. S. HORN (Ed.), Advances in Sport Psychology, pp. 181 -199. Champaign, IL: Human Kinetics Publishers.

HOTZ, A. (1997). Die Wettkampfdurchführung. In G. THIESS, P. TSCHIENE \& H. NICKEL (Hrsg.) Trainerbibliothek 33, Der sportliche Wettkampf. S. 112 -142.

HOTZ, A. (1990). Was zeichnet einen guten Trainer letzlich aus? In Leistungssport 20, 5, S. 45 ff.

HOWARD, A. \& BRAY, D. W. (1990). Prediction of managerial succes over long periods of time: Lessons from the Management Progress Study. In K. E. CLARK \& M. B. CLARK (Eds.), Measures of leadership (pp. 113 - 130). West Orange, NJ: Leadership Library of America.

ICKES, W. (1982). A basic paradigm for the study of personality, roles, and social behavior. In W. ICKES \& E. KNOWLES (Eds.), Personality, roles, and social behvior (pp. 305-341). New York: Springer.

ISELE, S. (1991). Managerleistung messen - beurteilen - honorieren. In K. KÄFER, P. WEILENMANN \& K. HÄSSIG (Hrsg.) Mitteilungen aus dem Handelswissenschaftlichen Seminar der Universität Zürich, Band 169. Zürich: Polygraphischer Verlag.

JÄGER, R. S. (1983). Der diagnostische Prozess. Eine Diskussion psychologischer und methodischer Randbedingungen. Göttingen: Hogrefe.

JUNG, C. G. (1921). Psychologische Typen. Olten: Walter.

KAPLAN, R. S. \& ATKINSON, A. A. (1989). Advanced Management Accounting. 2. Auflage. New Jersey: Eaglewood Cliffs.

KAYSER, G. (1987). Fitness. In Wörterbuch der Sportwissenschaft. Schorndorf: Hofmann.

KIM, K.-W., SCHARENBERG, S. \& KRÜGER, A. (1996). Wettkampf - Pädagogik - Kompetenz. Leistungssport 26, 5, S. 11 - 14, 30.

KLÄSS, K. (1989). Schulsport und Schülereinstellung. Ahrensburg. 
KLAUDI KLAUSEN, K. (1989). Fungerer idraettens organisationer? In Political Science Publications from Department of Commercial Law and Political Science. Nr. $7 / 89$.

KLINE, P. (1993). Personality. The psychometric view. London: Routledge.

KOMPA, A. (1995). Der gesellschaftliche und ideologische Kontext der Managementdiagnostik: Anmerkungen eines Kritikers. In W. SARGES (Hrsg.) Management-Diagnostik (S. 1 - 21). Göttingen: Hogrefe.

KRAUS, G. \& WESTERMANN, R. (1995). Projektmanagement mit System. Wiesbaden: Gabler.

KRÜGER, A. (1980). Das Berufsbild des Trainers im Sport. Schorndorf: Hofmann.

KRÜGER, A. (1981). Sport und Gesellschaft. Berlin: Tischler.

KRÜGER, A. (1989). Trainer brauchen Pädagogik! Eine Stellungnahme zum Beitrag von Cachay/Gahai: Brauchen Trainer Pädagogik? Leistungssport, 19, 6, S. 31 - 33.

KRÜGER, A. (1991). Hat sich die Sportpädagogik aus dem Leistungssport verabschiedet? Leistungssport, 21, 6, S. 15 - 18.

KRÜGER, A. (1995). Die Rolle der Trainerschaft in der Gesellschaft. Die Verantwortung für die Sportkultur. In KOZEL, J. (Hrsg.): Trainerakademie Köln e.V. 20 Jahre Trainerakademie, S. 80 - 91. Köln.

KUTLAR, M. (1999). Angebot und Umsetzung betreiblicher Gesundheitsförderung. Unveröffentlichte Diplomarbeit Umweltnaturwissenschaften, ETH Zürich.

LACY, O. W. (1984). Myers-Briggs Type Indicator and Strong-Campbell Interest Inventory. Unpublished raw data.

LORD, R. G., De VADER, C. L. \& ALLIGER, G. M. (1986). A meta-analysis of the relationship betwen personality traits and leadership perception: An application of validity generalization procedures. Journal of applied Psychology, 71, 402-410.

LUEDER, D. C. (1986). Psychological types and leadership styles of the 100 top executive educators in North America. Journal of Psychological Type, 12, pp. 8-12.

LUTHANS, F. \& LOCKWOOD, D. L. (1984). Toward an observational system for measuring leader behavior in natural settings. In J.G. HUNT, D. HOSKING, C.A. SSCHRIESHEIM \& R. STEWART (Eds.), Leaders and managers: International perspectives on managerial behavior and leadership (pp. 117 - 141). New York: Pergamon Press. 
LUTHANS, F., HODGETT, R. M. \& ROSENKRANTZ, S. (1988). Real Managers. Cambridge, MA: Balinger.

MANN, R. D. (1959). A review of the relationship between personality and performance in small groups. Psychological Bulletin, 56, 241 - 270.

MARCH, J. G. \& SIMON, H. A. (1976). Organisation und Individuum. Wiesbaden: Gabler.

MARTI, B. (1989). Physiologische, soziale und motivationale Charakteristika von Fitnesscenter-Besuchern. Schweizerische Zeitung für Sportmedizin 37, 4, Seiten 233239.

MARTI, B. (1997). Zeit zum Handeln. In Nachrichtenmagazin „Facts“, 29.

MARTI, B. \& MARTIN, B. (1998). Bewegung und Sport: eine unterschätzte Gesundheitsressource. Magglingen: SWI.

MARTIN, A. (1989). Die empirische Forschung in der Betriebswirtschaftslehre. Stuttgart.

MARTINKO, M. J. \& GARDNER, W. L. (1990a). The relationship between psychological type, managerial behavior and managerial effectiveness: an empirical investigation. Journal of Psychological Type. 19, pp. 34 - 41.

MARTINKO, M. J. \& GARDNER, W. L. (1990b). Structured observation for managerial work: A replication and sythesis. Journal of Management Studies. 12, pp. $43-48$.

MCALLISTER, L. (1990). Leadership -management style report. In L. A. Hammer (Ed.), Strong Interest Inventory topical reports manual (pp. 5-6). Palo Alto, CA: Consulting Psychologists Press.

MCAULEY, E. (1992). Self-referent thought in sport and physical activity. In Horn, T. S.: Advance in sport psychology. Champaign. Human Kinetics.

MCCAULLEY, M. H. (1977). Application of the Myers-Briggs Type Indicator to medicine and other health professions. Gainesville: CAP.

MCCRAE, R. R. \& COSTA P. T. (1987). Validation of the five factor model of personality across instruments and observers. Journal of Personality and Social Psychology, 52, pp. $81-90$.

MCGREGOR, D. (1970). Der Mensch im Unternehmen. Düsseldorf: Econ. 
MERTENS, A. \& VAHL, T. (1995). Fitnessanleitungsbücher: Von Sandow über Pearl bis in unsere Zeit. In A. KRÜGER und B. WEDEMEYER (Hrsg) Körperkraft Kraftkörper S. 32 - 51. Göttingen, Universitätsdruckerei.

MEYER, P. \& DAVIS, S. (1992). The CPI application guide: An essential tool for individual, group, and organizational development. Palo Alto, CA: Consulting Psychologists Press.

MILLS, C. J. (1983). Personality characteristics of gifted adolescents and their parents: Comparisons and implications for achievement and counseling. Paper presented at the Annual Meeting of American Educational Research Association. Montreal.

MILLS, J., ROBEY, D. \& SMITH, L. (1985). Conflict handling and personality dimensions of projectmanagement personnel. Psychological Reports, 57, pp. 11351143.

MINTZBERG, H. (1973). The nature of managerial work. New York: Harper \& Row.

MISCHEL, W. (1973). Toward a cognitive social learning reconceptualization of personality. Psychological Review, 80, 252-283.

MOCK, T. J. (1976). Measurement and Accounting Information Criteria. In Studies in Accounting Research, Volume 13. Sarasota, Florida: American Accounting Association Press.

MOSER, K. (1991). Konsistenz der Person. Göttingen: Hogrefe.

MYERS, I. (1962). The Myers Briggs Type Indicator. Palo Alto: Consulting Psychologist Press..

NEUBERGER, O. (1990). Führen und geführt werden. Stuttgart: Enke.

NIEDER, P. \& SUSEN, B. (Hrsg.).(1997). Betriebliche Gesundheitsförderung Konzepte und Erfahrungen bei der Realisierung. Bern.

OSTRENGA, M. R. (1990). Activities: The Focal Point of Total Cost Management. In Management Accounting, Feb, pp. 42 - 49.

PAFFENBARGER, R. S. (1989). Longterm Health Benefits of Lifestyle Elements of Harvard College Students. In Stanford Health and Exercise Handbook. Champaign IL: Leisure Press.

PERVIN, L. A. (1989). Persons, situations, interactions: The history of a controversy and a discussion of theoretical models. Academy of Managmenet Review, 14, 350-380. 
PETRAY, C. K. \& CORTESE, P. A. (1988). Physical Fitness: A Vital Component of the School Health Education Curriculum. In Health Education, Vol 19, 5, pp. 4 -7.

QUALITOP (1999). Presse-Dokumentation. Winterthur: Qualitop.

REDDIN, W. J. (1981). Das 3-D-Programm. Zur Leistungssteigerung des Managements. Landdsberg: Moderne Industrie.

REHKUGLER, H. (1997). Erfolgsfaktoren im Gastgewerbe. Zürich: Polygraphischer Verlag.

RIPPE, J. M. (1989). Dr. James Rippe's Fit for Succes: Proven Strategies for Executive Health. New York: Prentice-Hall.

RITTNER, V. \& MRAZEK, J. (1986). Bericht zum Forschungsbericht: Sport, Fitness und Aussehen. Köln: Deutsche Sporthochschule.

ROACH, B (1986). Organizational decision makers: Different types for different levels. Journal of Psychological Type, 12, pp 16 - 24.

ROSENTIEL, L. VON, MOLT, W. \& RÜTTINGER, B. (1988). Organisationspsychologie (7. Auflage). Stuttgart: Kohlhammer.

ROSENTSIEL, L. VON (1993). Kommunikation und Führung in Arbeitsgruppen. In H. Schuler (Hrsg.), Lehrbuch Organisationspsychologie (S. 321-351). Bern: Huber.

ROTTER, J. B. (1972). An introduction to social learning theory. New York: Holt, Rinehart \& Winston.

RÜHLI, E. (1985). Unternehmensführung und Unternehmenspolitik 1 (2. Auflage). Bern: Haupt.

SALOMON, L. \& ANHEIER, H. (1996). The Emerging Sector. Manchester.

SAMPLE-INSTITUT (1984). Sport und Gesundheit. Eine Untersuchung im Auftrag der Hamburg-Mannheim-Stiftung für Informationsmedizin e.V. Mölln: Eigenverlag.

SACK, H.-G. (1975). Sportliche Betätigung und Persönlichkeit. Ahrensburg.

SAGE, G. H. (1973). The Coach as Management: Organizational leadership in American sport. Quest, 19, pp. $35-40$.

SARGES, W. (1995). Eignungsdiagnostische Überlegungen für den Managementbereich. In W. SARGES (Hrsg.) Management-Diagnostik (S. 1 - 21). Göttingen: Hogrefe. 
SIX, B. (1975). Die Relation von Einstellung und Verhalten. In Zeitschift für Sozialpsychologie 6, S. $270-296$.

SCHEIN, E. H. (1980). Organisationspsychologie. Wiesbaden: Gabler.

SCHMID, D. (1997). EXPO 2001 Sinnvoll. Ein Projekt für Sinneserlebnisse an der Schweizerischen Landesausstellung 2001. Projektbeschrieb.

SCHMID, D. (1999a). Ideenskizzen 2005 für das Migros Sportzentrum Greifensee. Unveröffentlichte Studie im Auftrag des Migros Genossenschaftsbundes.

SCHMID, D. (1999b). Persönlichkeitstypen von Sportstudierenden der ETH Zürich. Unveröffentlichte Studie.

SCHNABEL, G. (1993). Fitness. In Lexikon der Sportwissenschaft. Schorndorf: Hofmann.

SCHWEIZ TOURISMUS (1999). Factsheet Tourismus Schweiz 1998. Bern: ST.

SEILER, A. (1991). Marketing. Erfolgreiche Umsetzung in die Praxis. Zürich: Orell Füssli.

SIMON, H. A. (1945). Entscheidungsverhalten in Organisationen. Landsberg an der Lech.

SPRANGER, E. (1925). Lebensformen (5. Verb. Auflage). Halle: Niemeyer.

STAEHLE, W. H. (1991). Handbuch Management - Die 24 Rollen der exzellenten Führungskraft. Wiesbaden: Gabler.

STAEHLE, W. H. (1992). Unternehmer und Manager. Management revue, 3, 95-108.

STELTER, R. (1996). Du bist wie Dein Sport. Studien zur Entwicklung von Selbstkonzept und Identität. Schorndorf: Hofmann.

STEWART, R. (1967). Managers and their Jobs. London.

STOGDILL R. M. (1948). Personal factors associated with leadership. A survey of the literature. Journal of Psychology, 25, 35 -71.

STRICKER, L. J. \& ROSS J. (1964). A description and evaluation of the MyersBriggs Type Indicator. Research Bulletin RB, 62-6.

STRONG, E. K. Jr. (1943). Vocational Interests of Men and Woman. Stanford, CA: Stanford University. 
URSPRUNG, L. (1998). Arbeitsmarkt Sport Schweiz. GFS Schriften Sportwissenschaften 16. Zürich: GFS.

VELSOR, E. V. \& CAMPBELL, D. (1984). Pearson correlations: Myers-Briggs continous scores and California Personality Inventory.Unpublished raw data.

VOS STRACHE, C. (1979). Player's perceptions of leadership qualities for coaches. ResearchQuaterly, 50, pp. 94 - 102.

WEBER, W., SCHNIEDER, C., KORTLÜKE, N. \& HORAK, B. (1994). Die wirtschaftliche Bedeutung des Sports. Paderborn.

WICKER, A. W. (1969). Attitudes versus actions: The relationship of verbal and overt behavioral responses to attitude objects. In: Journal of Social Issues 21, pp. 41 -78.

WILLIMCZIK, Klaus, Hrsg. (1993). Die ATPA-D-Skalen. Eine deutsche Fassung der Skalen von Kenyon zur Erfassung der Einstellung gegenüber sportlicher Aktivität. Limpert.

WOERMANN, S. \& HANKE, U. (1994). Expertentum bei Trainern. Leistungssport 18, 2, S. $10-14$. 


\section{Anhang}

Fragebogen mit den vier Bausteinen Persönlichkeit, Führungsverhalten, Situation und Erfolg. 